\title{
Using Mass Spectrometry to Measure the Effects of Low Dose Radiation Exposure on the Liver Lipidome
}

by

\author{
Frank Wong
}

A thesis submitted to the Faculty of Graduate and Postdoctoral Affairs in partial fulfillment of the requirements for the degree of

\author{
Master of Science \\ in \\ Chemistry \\ Carleton University \\ Ottawa, Ontario
}

○ 2021, Frank Wong 


\section{Author's Declaration}

I hereby declare that this thesis consists of materials all of which I authored or coauthored. While I am the sole author of this thesis, I acknowledge the contribution of Dr. Jeffrey $\mathrm{C}$. Smith in assisting with editing and providing feedback for my thesis manuscript. This is a true copy of the thesis, including any required final revisions, as accepted by my examiners.

I understand that my thesis may be made electronically available to the public. 


\section{Abstract}

For years it has been widely accepted that low dose radiation (LDR) exposure is completely harmless to humans, however this may not be true. There is growing evidence to suggest that LDR exposure may result in radiogenic effects. Although LDR may not result in direct cell death, mutations may occur and initiate cancerous behaviour. Previous studies suggest that continuous exposure to LDR may implicate significant changes in overall lipid metabolism, especially in the liver. In this study, liquid chromatography-mass spectrometry (LC-MS) methods were developed to explore the possible lipidomic effects of LDR through systemic $\alpha$-radiation exposure via injection of ${ }^{210} \mathrm{Po}$ in male Wistar rats and measuring the relative changes in lipid composition in their livers. A series of lipid extractions were performed using rat livers of different dosage levels via a modified BlighDyer method which were subsequently analyzed via reverse phase-high performance liquid chromatography mass spectrometry (RP-HPLC MS) in positive ion mode. Low dose exposure from an $\alpha$-emitter was of specific interest since there are currently no reported discoveries regarding its effects on a cellular lipidome. This study aids to provide comprehensive analysis of any potential lipidomic effects due to LDR. Here, our results demonstrate dynamic changes in the rat liver lipidome post-exposure to low dose radiation. Significant increases of many triacylglycerols and phosphatidylcholine lipid species were observed in the high and medium dose categories. Furthermore, significant decrease of triacylglycerols were observed in the low dose category. These dynamics are postulated to be involved with inflammatory response based on literature findings. Future studies are needed to explore LDR exposure effects upon other classes of lipids that are typically identified in negative ion mode. 


\section{Acknowledgments}

I would like to extend my sincere gratitude and thank you to my supervisor Professor Jeffrey C. Smith for the extraordinary opportunity to pursue a master's project unlike any other. Jeff, I am truly thankful for all the encouragement, guidance, and support despite the difficult challenges we've faced. Through a fire, a flood, and a global pandemic, you have continuously been a positive light to what seems to be a neverending abyss. We made it! I am forever indebted to your confidence and kindness. To my collaborator, Dr. Baki Sadi, I would like to convey my appreciativeness to you for the countless support and trust you have bestowed upon me to make this project a success. Your expertise and professionalism have made this one of the most rewarding experiences in my life. Thank you for always being patient and understanding, I am truly grateful for this opportunity.

To the foundation of the Carleton Mass Spectrometry Centre, Karl V. Wasslen. You have played an integral part of my success. It has been an honour to learn from someone who understands mass spectrometry as well as you do. Thank you for being a wonderful mentor and without you, I would not be where I am today. To a wonderful professor, Jeffrey M. Manthorpe. Jeff, thank you for all the encouragement and advice you have conveyed over the years (even if we only meet during lab meetings). You may not know how impactful your words are, but I truly value all the uplifting remarks and support.

Additionally, I am eternally grateful to both Dr. Samuel W.J. Shields and Joshua A. Roberts. Sam, no amount of words can express how grateful I am to learn from you. Even in a short time, you have been tremendously patient, kind and understanding. Thank you 
for the amazing support you have provided. Josh, where do I begin? I am extremely fortunate to have met you. Without you, I would not be where I am today. You have been an incredibly valuable resource for mass spectrometry and lipidomics. There are many nights where I sit down and ponder how I can pull this ambitious project off. You have been integral to the success, and I can't thank you enough for all the encouragement, advice and support. Rock on!

My gratitude extends to the fellow members of the Smith Lab, past and present. I would like to thank you all for the heart-warming friendships and experiences you have shared with me throughout my time here. To all the members of the Chemistry Department at Carleton University and members in Health Canada's Radiation Protection Bureau, thank you for the wonderful opportunities and support. In addition, I would also like to acknowledge the contributions made by the Canadian Nuclear Laboratories (Chalk River, Ontario, Canada) for making this research possible by carrying out the animal experiments in their Biological Research Facility.

To my wonderful friends, I would like to express my most sincere gratitude for the limitless support, encouragement, and love. You have made my experience truly unforgettable. A personal thank you to Brandon, Vernon, Duale, Dorothy and, James. I am immensely fortunate to have you all as a friend. I am thankful for all the wonderful memories and ever-lasting friendship.

To my biggest supporters, my parents. Thank you for absolutely everything. I cannot begin to describe how grateful I am to be loved by you both. Thank you for always believing in me and listening to the countless rants I make. You both have always encouraged me to take my knowledge and abilities to the next level and to step out of my 
boundaries. Mom, dad, you both have been an inspiration to me. Although conveying my research (or really any science) is way beyond the scope of your understanding, I am appreciative to have someone who will listen and comprehend what I do. I have both of you to thank for my drive and determination to obtain what is a wonderful opportunity and fulfilling education. 爸爸媽媽, 我愛你!

To my girlfriend, Cindy. I can't thank you enough for the endless encouragement and support you have provided throughout this journey. Thank you for your positivity and unconditional love despite being incredibly long distant. You have made every moment worthwhile and all I can say is thank you! I will forever cherish your lovely quote "Lipids makes peaks. BAM! Thesis!" I love you \&.

Lastly, I would like to thank Carleton University, the Department of Chemistry, and Health Canada for providing the funding and a unique opportunity. 


\section{Table of Contents}

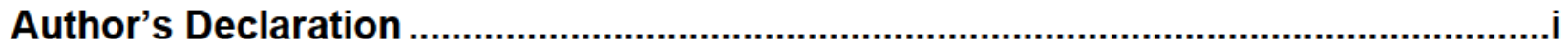

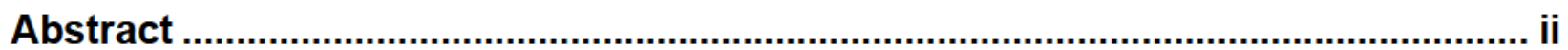

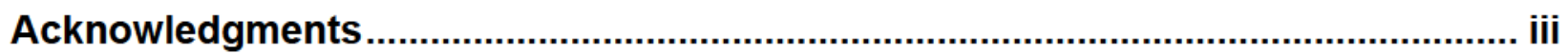

List of Tables.................................................................................................. viii

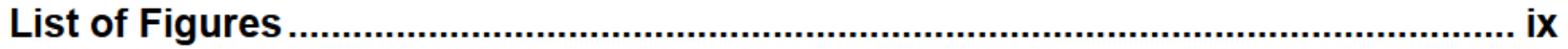

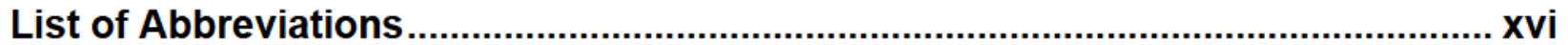

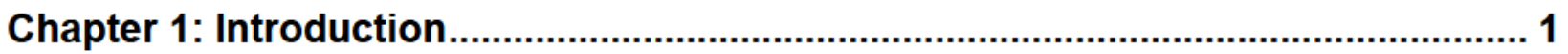

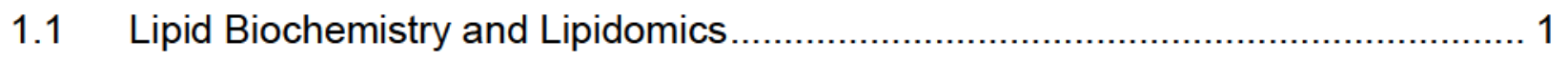

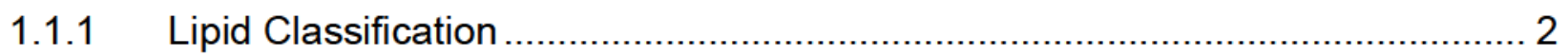

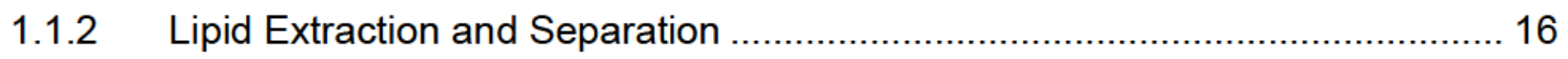

1.1.3 Lipid Analysis Before Mass Spectrometry ............................................... 17

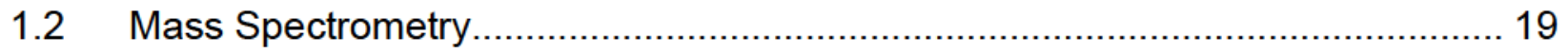

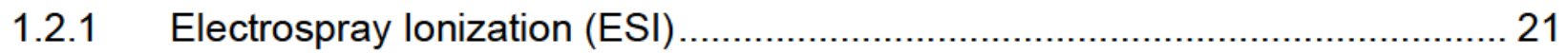

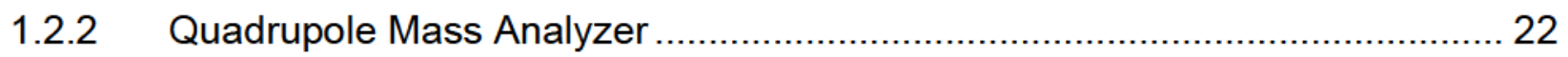

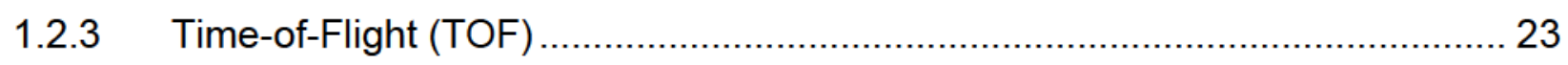

1.2.4 Ion Detector: Multichannel Plate Detector ................................................ 27

1.2.5 Hybrid Quadrupole Time-of-Flight Mass Spectrometer ................................... 28

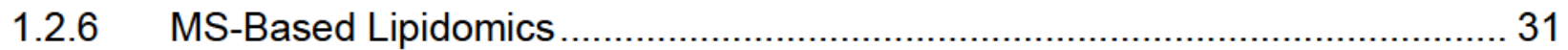

1.3 Separation via High Performance Liquid Chromatography ................................. 33

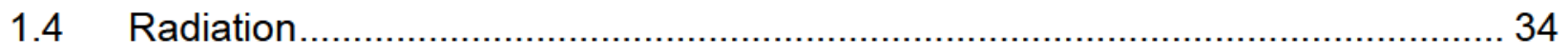

1.4.1 Various Types of Radiation (ionizing/non-ionizing) …................................. 35

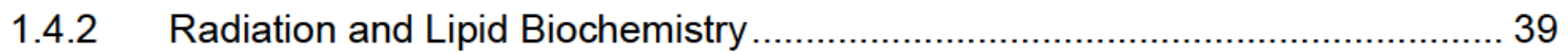

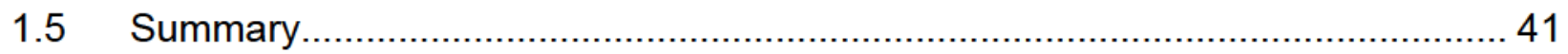

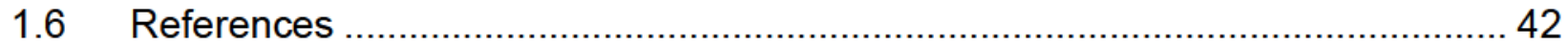

Chapter 2: Materials and Methodology ............................................................... 51

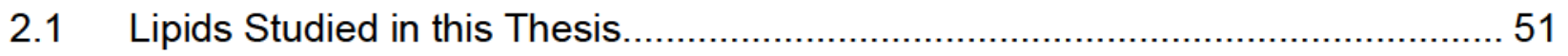

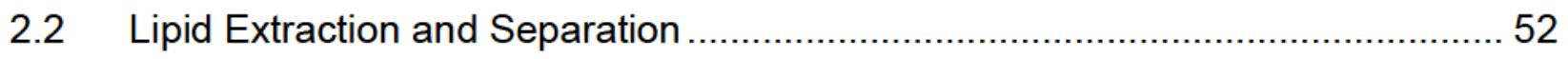

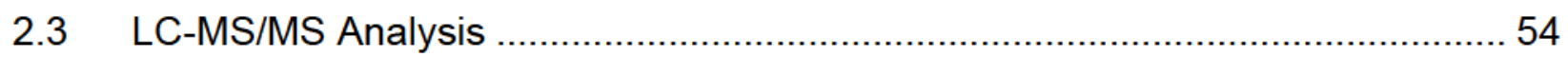

2.4 Post-Data Acquisition (Agilent Software) ……........................................ 57

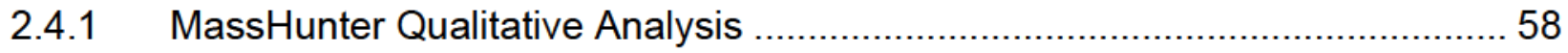

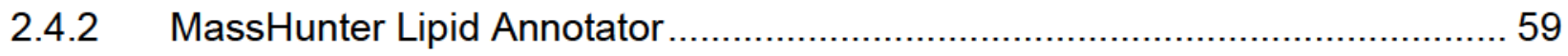


2.4.3 Personal Compound Database \& Library (PCDL) Manager ........................... 61

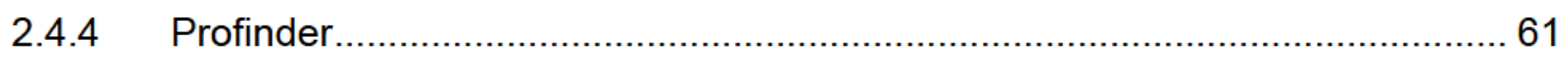

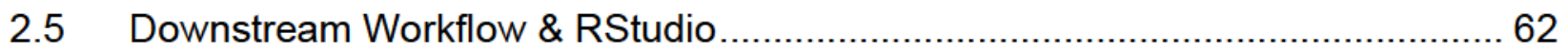

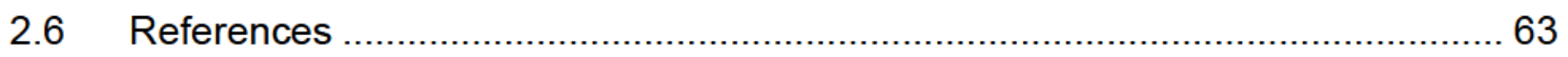

Chapter 3: Optimization and Developmental Experiments Prior to Analysis of Rat Liver Samples using Bovine and Control Rat Liver Tissues ....................................65

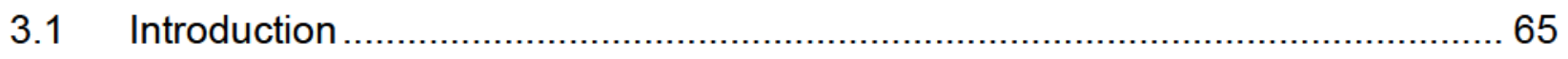

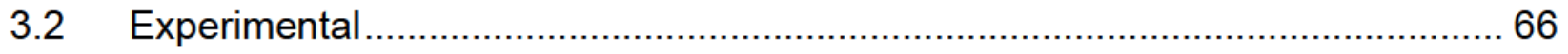

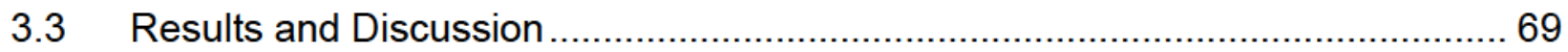

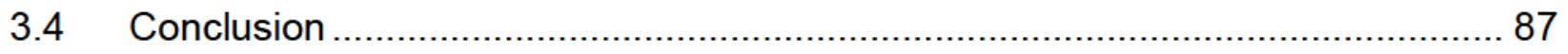

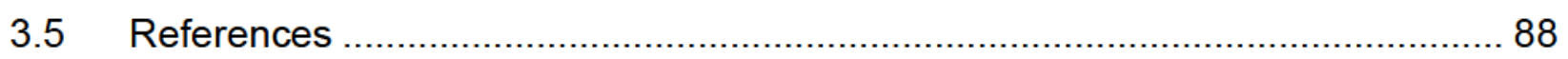

Chapter 4: Analysis of Low-Dose Radiation Exposed Rat Liver.............................90 90

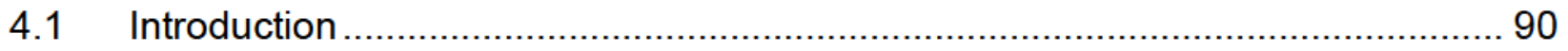

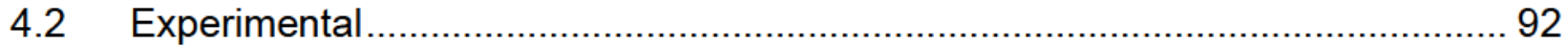

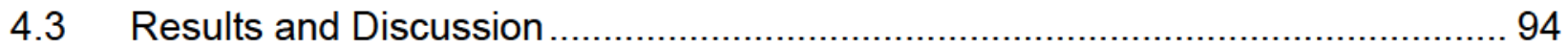

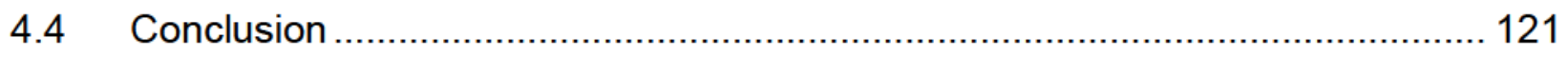

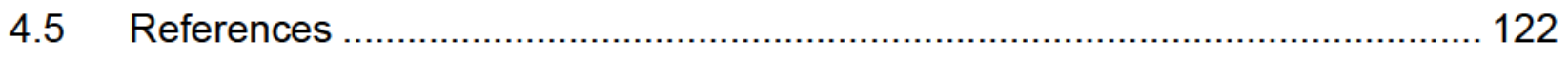

Chapter 5: Conclusion and Future Works.......................................................... 125

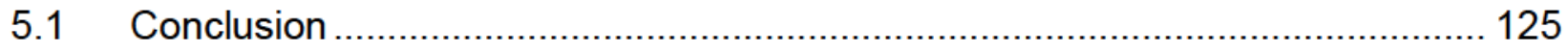

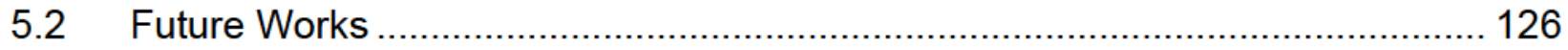

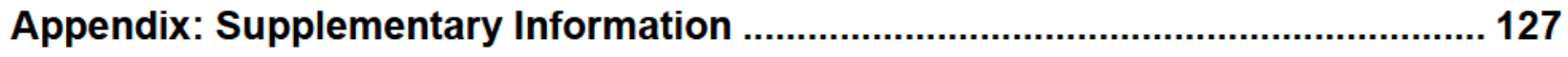




\section{List of Tables}

\section{Chapter 3}

Table 3.1: PCs annotated from Lipid Annotator using gradient programs A, B and C... 74

Table 3.2: Retention time of lipid species extracted from bovine liver diluted 5-fold in $50 \% \mathrm{MeOH}, 50 \% \mathrm{EtOH}$ and in $80 \% \mathrm{MeOH}, 20 \% \mathrm{EtOH}$.

\section{Chapter 4}

Table 4.1: The tissue sample used for lipid extractions. Sample was randomized by Microsoft Excel's randomization function. Notation as follows: C = control, D1 = Low Dose, D2 = Med Dose, D3 = High Dose. The number after indicates the liver sample. 93

Table 4.2: Mass of wet liver tissue (in $\mathrm{mg}$ ) used in each extraction. Target mass was 1 $\mathrm{mg}$. However, since some samples provided did not contain a total of $3 \mathrm{mg}$ (wet tissue weight), therefore some recorded masses are lower than target weigh. Liver tissues were weighed on a Mettler AT250 analytical balance.

Table 4.3: Lipids showing significant increase in fold change between the high dosed livers versus control livers.

Table 4.4: Lipids showing significant decrease in fold change between the high dosed livers versus control livers. 106

Table 4.5: Lipids showing significant increase in fold change between the medium dosed livers versus control livers.

Table 4.6: Lipids showing significant decrease in fold change between the medium dosed livers versus control livers.

Table 4.7: Lipids showing significant decrease in fold change between the low dosed livers versus control livers. 


\section{List of Figures}

\section{Chapter 1}

Figure 1.1: Diagram of a cell membrane. Adapted from Biodictionary.net ${ }^{13} \ldots \ldots \ldots \ldots \ldots \ldots . . .1$

Figure 1.2: Representative structures of the 8 lipid classes. Adapted from Bou Kahalil et al. $^{2}$ 3

Figure 1.3: Representative structures of 5 fatty acyl classes. Adapted from Bou Kahalil et al. ${ }^{2}$

Figure 1.4: Representative structures of 5 glycerolipid classes. Adapted from Eoin Fahy et al. ${ }^{16}$

Figure 1.5: Representative structures of 4 sterol classes. Adapted from Eoin Fahy et al. ${ }^{16}$

Figure 1.6: Representative structures of 4 prenol classes. Adapted from Eoin Fahy et al. ${ }^{16}$

Figure 1.7: Representative structures of 2 saccharolipid classes. Adapted from Eoin Fahy et al. ${ }^{16}$

Figure 1.8: Representative structures of 4 polyketide classes. Adapted from Eoin Fahy et al. ${ }^{16}$

Figure 1.9: Representative structures of 20 glycerophospholipid classes. Adapted from Bou Kahalil et al. ${ }^{2}$

Figure 1.10: Representative structures of the 10 sphingolipid classes. Adapted from Bou Kahalil et al. ${ }^{2}$.

Figure 1.11: Fundamental components of a mass spectrometer.

Figure 1.12: Schematic of electrospray ionization. Figure adapted from Harris, D. C. $9^{\text {th }}$ Ed. ${ }^{40}$

Figure 1.13: Schematic of a quadrupole. Figure adapted from Shimadzu Scientific Korea Corp. ${ }^{60}$

Figure 1.14: Schematic of a TOF. Figure adapted from Harris, D. C. $9^{\text {th }}$ Ed. ${ }^{40}$ 24

Figure 1.15: Different non-uniform conditions that decreases resolution in a TOF-MS. The $U$ and $L$ designates two ions with identical $\mathrm{m} / \mathrm{z}$. A) Not starting at the same point in the ion accelerator, resulting in different velocities in the drift tube. This results in the $U$ 
ion to hit the detector later than $L$. B) lon $L$ starts off with more residual velocity than $U$, resulting in $L$ hitting the detector earlier than ion $U$. C) Pulsing of the ion accelerator causes ion $L$ to travel the other way. This results in ion $U$ hitting the detector earlier. Figure adapted from Smith. ${ }^{64}$.....

Figure 1.16: Schematic of how a reflectron or ion mirror corrects for the different spatial distribution with identical $\mathrm{m} / \mathrm{z}$ ions. Figure adapted from Smith. ${ }^{64}$ 27

Figure 1.17: Scheme of a multichannel plate detector. Adapted from Smith. ${ }^{64}$ 28

Figure 1.18: A) Scheme of two microchannel plates and a cascade of electrons from one electron emission. Adapted from Eberhard Karls Universität Tübingen, Faculty of Science, Institute for Astronomy and Astrophysics. ${ }^{66}$ B) Cross-section of a CEM.

Adapted from Penlink. ${ }^{67}$

Figure 1.19: Schematic of Agilent's 6546 LC-QTOF. Figure adapted from Agilent Technologies (user manual). ${ }^{68}$

Figure 1.20: Schematic of Agilent's dual Jet Stream Technology electrospray ionization source. Figure adapted from Agilent Technologies (user manual). ${ }^{68}$ 30

Figure 1.21: Electromagnetic Spectrum. Figure adapted from University of Washington, Environmental Health \& Safety. ${ }^{83}$ 36

Figure 1.22: Penetrating power of different types of radiation. Figure adapted from Jan Helebrant. ${ }^{84}$.

Figure 1.23: Relationship between radioactive decay and detection. Figure obtained from Wikipedia. ${ }^{89}$

Figure 1.24: Relationship between radiation decay and dosage. Figure obtained from Ministry of the Environment, Government of Japan. ${ }^{90}$

\section{Chapter 2}

Figure 2.1: Representative of the lipid classes focused on this thesis. Adapted from Bou Kahalil et al. ${ }^{1}$ and Eoin Fahy et al. ${ }^{2}$

Figure 2.2: A binary gradient program (gradient program A) with a constant flow rate $(0.4 \mathrm{~mL} / \mathrm{min})$ for lipid elution was used. Solvent A consisted of $50 \% \mathrm{MeOH}, 50 \% \mathrm{H}_{2} \mathrm{O}$ with $10 \mathrm{mM} \mathrm{NH}_{4} \mathrm{HCO}_{2}$ and solvent $B$ consisted of $75 \%$ IPA, $25 \% \mathrm{MeOH}$ with $10 \mathrm{mM}$ $\mathrm{NH}_{4} \mathrm{HCO}_{2}$

Figure 2.3: An excerpt from the worklist with internal standard quality control (QC) injections. 
Figure 2.4: A general untargeted workflow for lipid analysis utilizing Agilent's Lipid Annotator. Figure adapted from Agilent Technologies. ${ }^{6}$.....

Figure 2.5: A general untargeted workflow for lipid analysis using a suite of Agilent software and RStudio. Figure adapted from Klein, C. \& Sartain, M. ${ }^{6}$

Figure 2.6: Lipid Annotator's feature finding Q-score parameter was set to Q-score lower than 30.0. Q-score is defined as the chromatographic peak quality. Any feature that form a very poor chromatographic peak was filtered out of the feature list......

Figure 2.7: Both polarities were selected including all common adducts. The following lipid classes were selected for annotation.

Figure 2.8: Identification parameters were set in Lipid Annotator to reduce the amount of falsely identified lipids 60

Figure 2.9: An example of a lipidomics database in PCDL

\section{Chapter 3}

Figure 3.1: Gradient program $B$ with a constant flow rate $(0.4 \mathrm{~mL} / \mathrm{min})$ for lipid elution was used. Solvent A consisted of $50 \% \mathrm{MeOH}, 50 \% \mathrm{H}_{2} \mathrm{O}$ with $10 \mathrm{mM} \mathrm{NH}_{4} \mathrm{HCO}_{2}$ and solvent $\mathrm{C}$ consisted of $25 \%$ IPA, $75 \% \mathrm{MeOH}$ with $10 \mathrm{mM} \mathrm{NH}_{4} \mathrm{HCO}_{2}$

Figure 3.2: Gradient program $C$ with a constant flow rate $(0.4 \mathrm{~mL} / \mathrm{min})$ for lipid elution was used. Solvent $\mathrm{A}$ consisted of $50 \% \mathrm{MeOH}, 50 \% \mathrm{H}_{2} \mathrm{O}$ with $10 \mathrm{mM} \mathrm{NH}_{4} \mathrm{HCO}_{2}$, solvent B consisted of $75 \%$ IPA, $25 \% \mathrm{MeOH}$ with $10 \mathrm{mM} \mathrm{NH}_{4} \mathrm{HCO}_{2}$ and solvent $\mathrm{C}$ consisted of $25 \%$ IPA, $75 \% \mathrm{MeOH}$ with $10 \mathrm{mM} \mathrm{NH}_{4} \mathrm{HCO}_{2}$.

Figure 3.3: Relative distribution of lipids found in bovine liver using gradient program A

Figure 3.4: Relative distribution of lipids found in bovine liver using gradient program B

Figure 3.5: Relative distribution of lipids found in bovine liver using gradient program C

Figure 3.6: A $\mathrm{m} / \mathrm{z}$ vs time (mins) plot of a 5-fold diluted bovine liver extract using gradient program $A$.

Figure 3.7: A m/z vs time (mins) plot of a 5-fold diluted bovine liver extract using gradient program $B$

Figure 3.8: A m/z vs time (mins) plot of a 5 -fold diluted bovine liver extract using gradient program $\mathrm{C}$ 
Figure 3.9: Number of lipids identified in each lipid class using gradients A, B and C from bovine liver.

Figure 3.10: EIC of the internal standard LPC C13:0 in all three gradient programs from a bovine liver extract. Grey: Gradient A, Red: Gradient B, Green: Gradient C. 77

Figure 3.11: ESI+ MS and MS/MS spectra of LPC C13:0 (m/z 454.29) using gradient program A. Blue diamond represents the parent ion.

Figure 3.12: $\mathrm{ESI}+\mathrm{MS}$ and MS/MS spectra of LPC C13:0 (m/z 454.29) using gradient program $\mathrm{B}$. Blue diamond represents the parent ion.

Figure 3.13: $\mathrm{ESI}+\mathrm{MS}$ and MS/MS spectra of LPC C13:0 (m/z 454.29) using gradient program $\mathrm{C}$. Blue diamond represents the parent ion.

Figure 3.14: A m/z vs time (mins) plot of a 2-fold diluted rat liver extract using gradient program $A$

Figure 3.15: A m/z vs time (mins) plot of a 2-fold diluted rat liver extract using gradient program $B$

Figure 3.16: A m/z vs time (mins) plot of a 2-fold diluted rat liver extract using gradient program $\mathrm{C}$

Figure 3.17: Number of lipids identified in each lipid class using gradients A, B and C from rat liver.

Figure 3.18: Relative distribution of lipids found in bovine liver using gradient program $A$ with $A$ ) iterative injection and $B$ ) single injection 86

\section{Chapter 4}

Figure 4.1: Structure of internal standard LPC C13:0 (453.29 Da).

Figure 4.2: Extracted ion chromatogram of $\mathrm{m} / \mathrm{z} 454.2940,[\mathrm{M}+\mathrm{H}]^{+}$of LPC 13:0/0:0 (internal standard) from twenty nine $5 \mu \mathrm{L}$ injections of $0.7 \mu \mathrm{M}$ internal standard..

Figure 4.3: Extracted ion chromatogram of $\mathrm{m} / \mathrm{z} 454.2940,[\mathrm{M}+\mathrm{H}]^{+}$of LPC 13:0/0:0 (internal standard) from fifteen $5 \mu \mathrm{L}$ injections (5 extracted spiked samples, each in triplicate).

Figure 4.4: Distribution of lipid classes found in rat liver across all four dosage categories. There are a total of 214 lipids found in 20 rat liver samples that were fully identified by Agilent's Lipid Annotator and quantified using Agilent's Profinder. 
Figure 4.5: Heatmap with hieratical clustering of all the rat livers and lipid species identified in this experiment. Colours on the top represent dosage levels (control = green, low $=$ purple, medium $=$ magenta, and high $=$ orange $)$.

Figure 4.6: Heatmap of all the rat livers used in this experiment without hierarchical clustering of the samples. Samples were organized from control, low, medium to high dosage.

Figure 4.7: PCA of all the rat livers used in this experiment. PCA describes $40.6 \%$ of the variation in this study. The ellipses represent $95 \%$ confidence with respect to the dosage group.

Figure 4.8: Correlation circle of all the lipids annotated from in this experiment. Magnitude of the arrow and colour illustrates correlations of each lipid to each of the two principal components. 102

Figure 4.9: Manhattan plot depicting fold change between the high versus control. Red line indicates the statistically significant line after applying the $\mathrm{BH}$ method. $\mathrm{X}$-axis indicates $\mathrm{m} / \mathrm{z}$.

Figure 4.10: Volcano plot depicting fold change between the high versus control. Red horizontal line indicates the statistically significant line after applying the $\mathrm{BH}$ method. Red vertical lines indicate $\log _{10}(\mathrm{FC})= \pm 0.5$.

Figure 4.11: Relative abundance of the top 10 lipids with the highest normalized peak area averages in rat liver control (blue) versus high (yellow) dose samples that increased in a statistically significant manner after applying an FDR correction. Low (orange) and medium (grey) dose levels were included to show the change in between the control and high dosage.

Figure 4.12: Relative abundance of the 26 lipids with normalized peak area averages in rat liver control (blue) versus high (yellow) dose samples that increased in a statistically significant manner after applying an FDR correction. Low (orange) and medium (grey) dose levels were included to show the change in between the control and high dosage.

Figure 4.13: Relative abundance of 5 lipids with normalized peak area averages in rat liver control (blue) versus high (yellow) dose samples that decreased in a statistically significant manner after applying an FDR correction. Low (orange) and medium (grey) dose levels were included to show the change in between the control and high dosage.

Figure 4.14: Manhattan plot depicting fold change between the medium versus control. Red line indicates the statistically significant line after applying the $\mathrm{BH}$ method. X-axis indicates $\mathrm{m} / \mathrm{z}$. 
Figure 4.15: Volcano plot depicting fold change between the medium versus control. Red horizontal line indicates the statistically significant line after applying the $\mathrm{BH}$ method. Red vertical lines indicate $\log _{10}(F C)= \pm 0.5$.

Figure 4.16: Relative abundance of the top 5 lipids with the highest normalized peak area averages in rat liver control (blue) versus medium (grey) dose samples that increased in a statistically significant manner after applying an FDR correction. Low (orange) and high (yellow) dose levels were included to show the changes in those categories.

Figure 4.17: Relative abundance of the 11 lipids with normalized peak area averages in rat liver control (blue) versus medium (grey) dose samples that increased in a statistically significant manner after applying an FDR correction. Low (orange) and high (yellow) dose levels were included to show the changes in those categories.

Figure 4.18: Relative abundance of the top 6 lipids with the highest normalized peak area averages in rat liver control (blue) versus medium (grey) dose samples that decreased in a statistically significant manner after applying an FDR correction. Low (orange) and high (yellow) dose levels were included to show the changes in those categories.

Figure 4.19: Relative abundance of the 15 lipids with normalized peak area averages in rat liver control (blue) versus medium (grey) dose samples that decreased in a statistically significant manner after applying an FDR correction. Low (orange) and high (yellow) dose levels were included to show the changes in those categories.

Figure 4.20: Manhattan plot depicting fold change between the low versus control. Red line indicates the statistically significant line after applying the $\mathrm{BH}$ method. $\mathrm{X}$-axis indicates $\mathrm{m} / \mathrm{z}$

Figure 4.21: Volcano plot depicting fold change between the low versus control. Red horizontal line indicates the statistically significant line after applying the $\mathrm{BH}$ method. Red vertical lines indicate $\log _{10}(\mathrm{FC})= \pm 0.5$

Figure 4.22: Relative abundance of the top 7 lipids with the highest normalized peak area averages in rat liver control (blue) versus low (orange) dose samples that decreased in a statistically significant manner after applying an FDR correction. Medium (grey) and high (yellow) dose levels were included to show the changes in those categories.

Figure 4.23: Relative abundance of the 13 lipids with normalized peak area averages in rat liver control (blue) versus low (orange) dose samples that decreased in a statistically significant manner after applying an FDR correction. Medium (grey) and high (yellow) dose levels were included to show the changes in those categories. 118 


\section{Supplementary}

Figure S1: Histogram plot of mean normalized intensity lipids that were acquired using the 6546 QTOF. Samples were analyzed using Agilent's Profinder and RStudio.

Figure S2: Histogram plot of mean normalized intensity lipids that were acquired using the 6546 QTOF. Samples were analyzed using Agilent's Profinder and RStudio. 127

Figure S3: Histogram plot of mean normalized intensity lipids that were acquired using the 6546 QTOF. Samples were analyzed using Agilent's Profinder and RStudio. 128

Figure S4: Histogram plot of mean normalized intensity lipids that were acquired using the 6546 QTOF. Samples were analyzed using Agilent's Profinder and RStudio.

Figure S5: Histogram plot of mean normalized intensity lipids that were acquired using the 6546 QTOF. Samples were analyzed using Agilent's Profinder and RStudio. 129

Figure S6: Histogram plot of mean normalized intensity lipids that were acquired using the 6546 QTOF. Samples were analyzed using Agilent's Profinder and RStudio. 129

Figure S7: Histogram plot of mean normalized intensity lipids that were acquired using the 6546 QTOF. Samples were analyzed using Agilent's Profinder and RStudio. 130

Figure S8: Histogram plot of mean normalized intensity lipids that were acquired using the 6546 QTOF. Samples were analyzed using Agilent's Profinder and RStudio. 130 


\section{List of Abbreviations}

$\begin{array}{ll}\% & \text { percentage } \\ \alpha & \text { alpha } \\ \beta & \text { beta } \\ \Delta & \text { delta } \\ \gamma & \text { gamma } \\ { }^{\circ} \mathrm{C} & \text { Celsius } \\ { }^{137} \mathrm{CS} & \text { cesium-137 } \\ { }^{14} \mathrm{C} & \text { carbon-14 } \\ { }^{20} \mathrm{Ne} & \text { neon-20 } \\ { }^{22} \mathrm{Ne} & \text { neon-22 } \\ { }^{206} \mathrm{~Pb} & \text { lead-206 } \\ { }^{210} \mathrm{Po} & \text { polonium-210 } \\ { }^{238} \mathrm{U} & \text { uranium-238 } \\ { }^{60} \mathrm{CO} & \text { cobalt-60 } \\ \mathrm{AC} & \text { alternating current } \\ \mathrm{ACC} & \text { Animal Care Committee } \\ \mathrm{ACMeOH} & \text { acidified methanol } \\ \mathrm{AJS} & \text { dual Jet Stream Technology } \\ \mathrm{APCl} & \text { atmospheric pressure chemical ionization } \\ \mathrm{BH} & \text { Benjamini-Hochberg } \\ \mathrm{Bq} & \text { Becquerel } \\ \mathrm{CCAC} & \text { Canadian Council on Animal Care } \\ \mathrm{CE} & \text { cholesteryl ester } \\ \mathrm{CEM} & \text { channel electron multiple } \\ \mathrm{Cer} \_\mathrm{NS} & \text { non-hydroxy fatty acid sphingosine ceramides } \\ \mathrm{CHCl} & \text { chloroform } \\ \mathrm{Cl} & \text { chemical ionization } \\ \mathrm{CID} & \text { collision-induced dissociation } \\ \mathrm{CNL} & \text { Canadian Nuclear Laboratory } \\ \mathrm{CNSC} & \text { Canadian Nuclear Safety Commission } \\ \mathrm{COX} & \text { cyclooxygenase } \\ \mathrm{CT} & \text { computed tomography } \\ \mathrm{Da}, \mathrm{mDa} & \text { Dalton, millidalton } \\ \mathrm{DC} & \text { direct current } \\ \mathrm{DG} & \text { diacylglycerol } \\ \mathrm{DNA} & \text { deoxyribonucleic acid } \\ \mathrm{EI} & \end{array}$




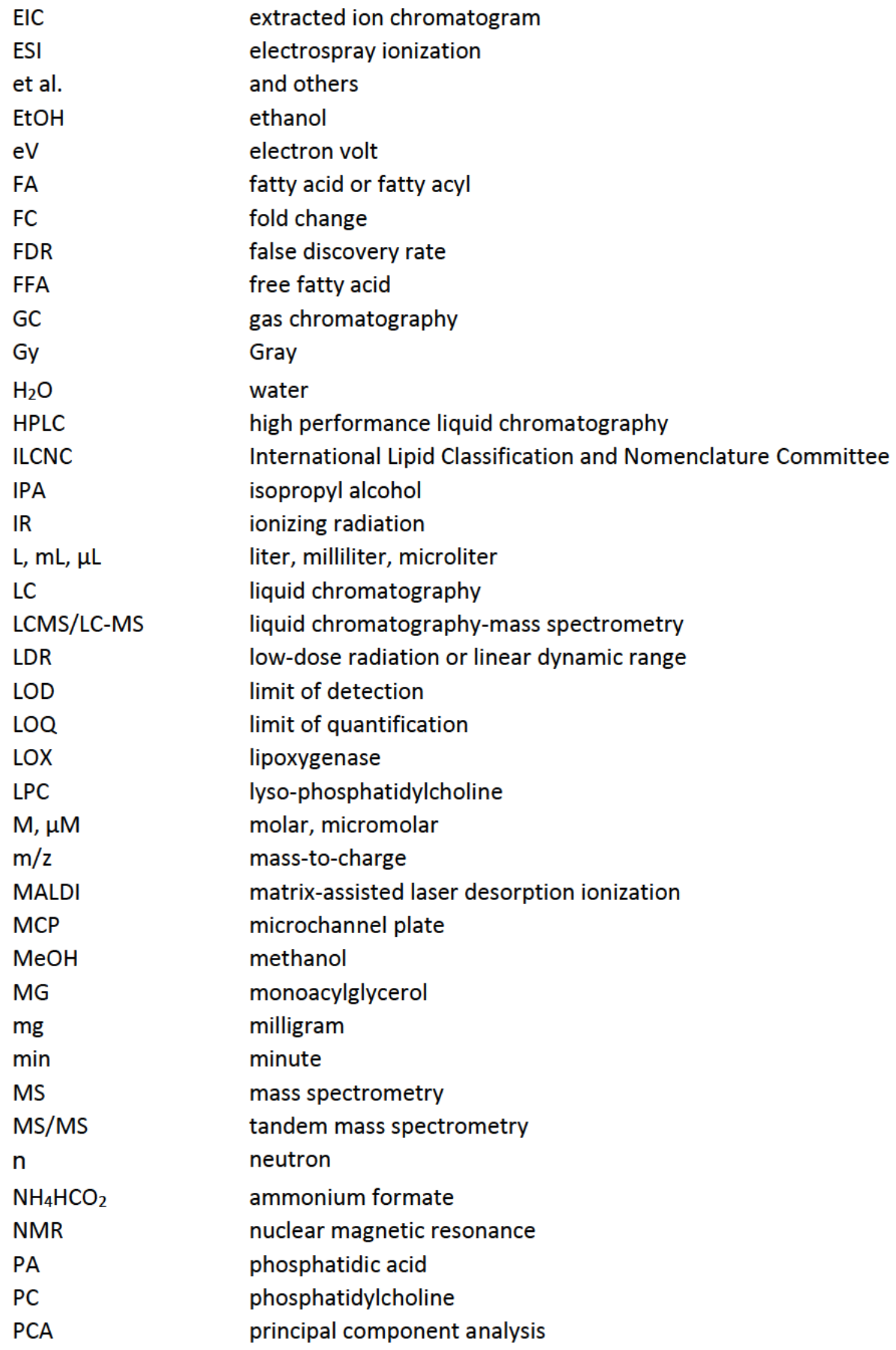




$\begin{array}{ll}\text { PCDL } & \text { Personal Compound Database and Library } \\ \text { PE } & \text { phosphatidylethanolamine } \\ \text { PG } & \text { phosphatidylglycerol } \\ \text { PI } & \text { phosphatidylinositol } \\ \text { ppm } & \text { parts per million } \\ \text { PS } & \text { phosphatidylserine } \\ \text { QC } & \text { quality control } \\ \text { QTOF, Q/TOF } & \text { quadrupole time-of-flight } \\ \text { RF } & \text { radio frequency } \\ \text { ROS } & \text { reactive oxygen species } \\ \text { RP-HPLC-MS } & \text { reverse phase-high performance liquid chromatography-mass } \\ \text { rpm } & \text { spectrometry } \\ \text { SI } & \text { revolutions per minute } \\ \text { SM } & \text { International System of Units } \\ \text { sn-1, sn-2, sn-3 } & \text { sphingomyelin } \\ \text { Sv } & \text { stereospecific numbering-1, stereospecific numbering-2, } \\ \text { TG } & \text { stereospecific numbering-3 } \\ \text { Th } & \text { Sievert } \\ \text { TLC } & \text { triacylglycerol } \\ \text { TOF,ToF } & \text { Thompson } \\ \text { V, kV } & \text { thin layer chromatography } \\ & \text { time-of-flight } \\ & \text { volt, kilovolt } \\ \end{array}$




\section{Chapter 1: Introduction}

\subsection{Lipid Biochemistry and Lipidomics}

Lipids are one of the fundamental macromolecules of cellular life. Commonly defined as substances of biological origin that are soluble in organic solvents ${ }^{1}$, they play essential roles in physiological functions and homeostasis. ${ }^{2}$ The most notable roles that lipids are involved in include energy storage within the cell, major structural components of the cell membrane, and intracellular and intercellular signalling. ${ }^{3,4}$ When aggregated together, lipids form macromolecular structures that compartmentalize cells based on their amphipathic properties. ${ }^{5}$ The field of lipidomics, a subsection of metabolomics that focuses on identification, quantification, and interactions of the complete lipid profile (lipidome) within a cell ${ }^{6}$, has been an increasing field of interest due to its involvement with numerous physiological diseases including diabetes ${ }^{7}$, obesity ${ }^{8}$, Alzheimer's ${ }^{9}$, stroke $^{10}$, cancer $^{11}$, and atherosclerosis ${ }^{12}$.

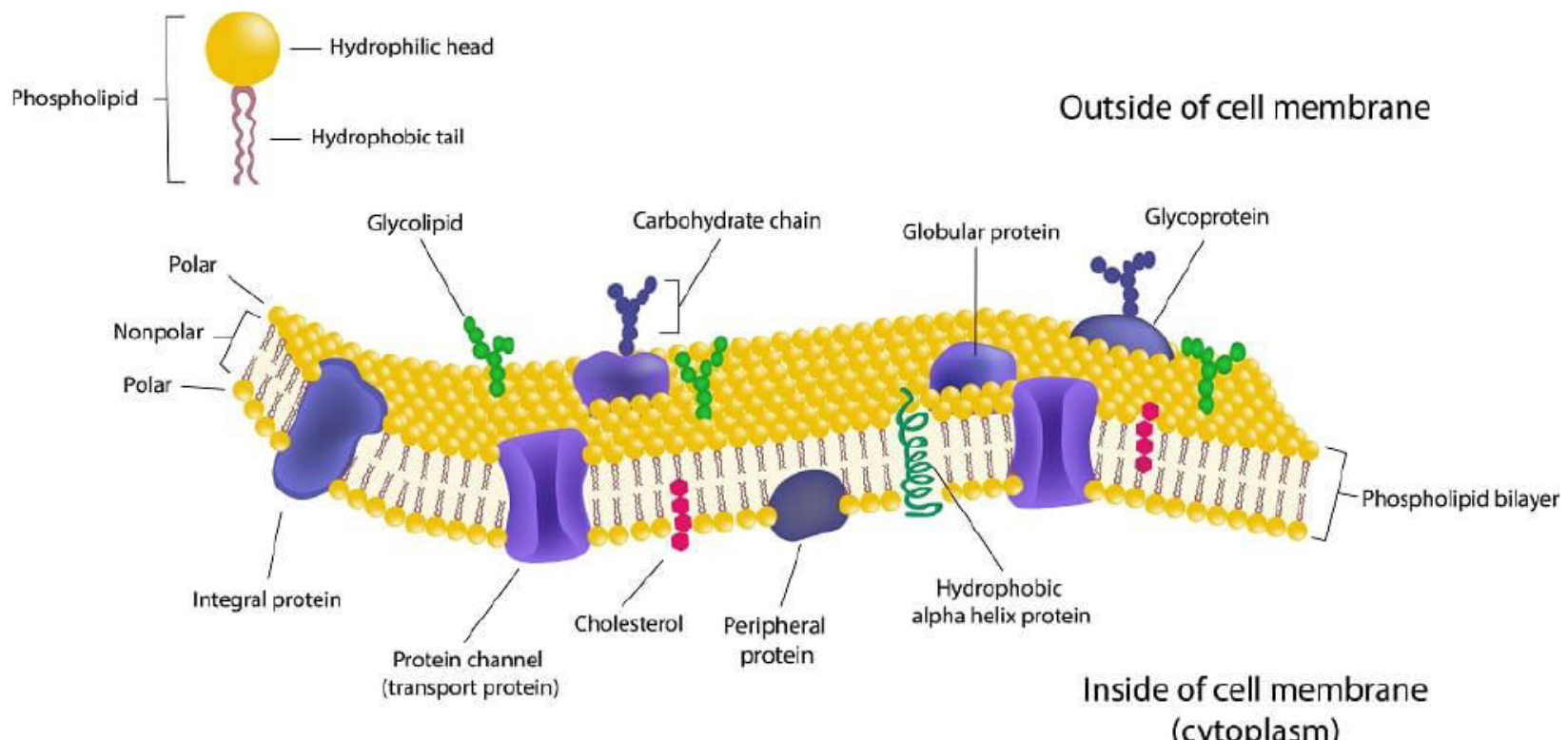

Figure 1.1: Diagram of a cell membrane. Adapted from Biodictionary.net ${ }^{13}$ 


\subsubsection{Lipid Classification}

With over 1000 lipid species in a cellular lipidome ${ }^{14}$ and a potential for $\sim 100,000$ unique molecular species ${ }^{15}$, a classification system was created. In 2005, Fahy et al. developed a comprehensive classification system comprised of eight major lipid classes ${ }^{16}$ illustrated in Figure 1.2. This classification system, which originally focused on mammalian lipids, has now expanded to include plants, bacteria, and fungi. ${ }^{3}$ Under the leadership of the International Lipid Classification and Nomenclature Committee (ILCNC), the eight distinct lipid classes are fatty acids, glycerolipids, glycerophospholipids, sphingolipids, sterols, prenols, saccharolipids, and polyketides. ${ }^{16}$ Each of these lipid classes provide a unique function within a biological system.

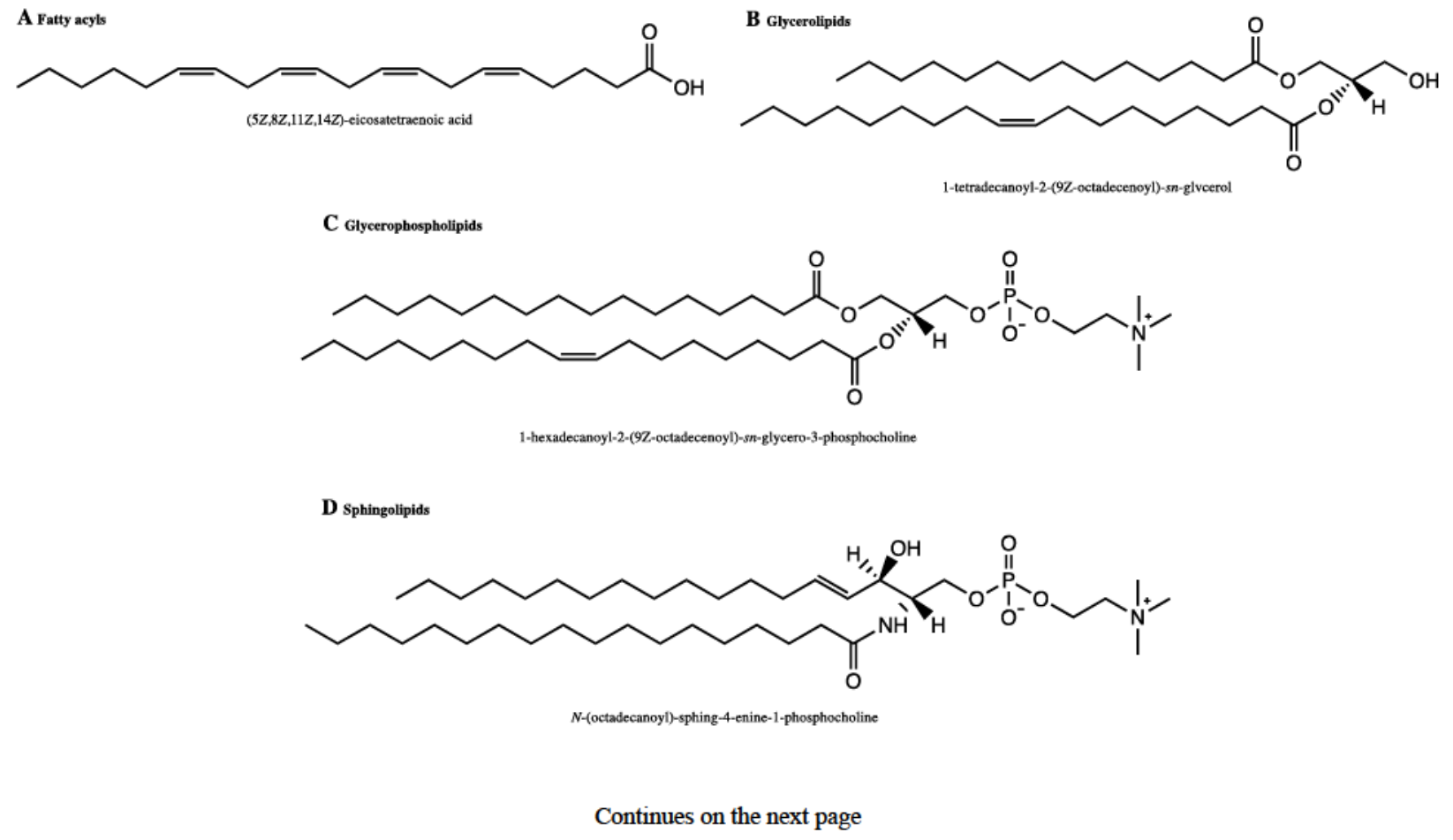


E Sterol lipids
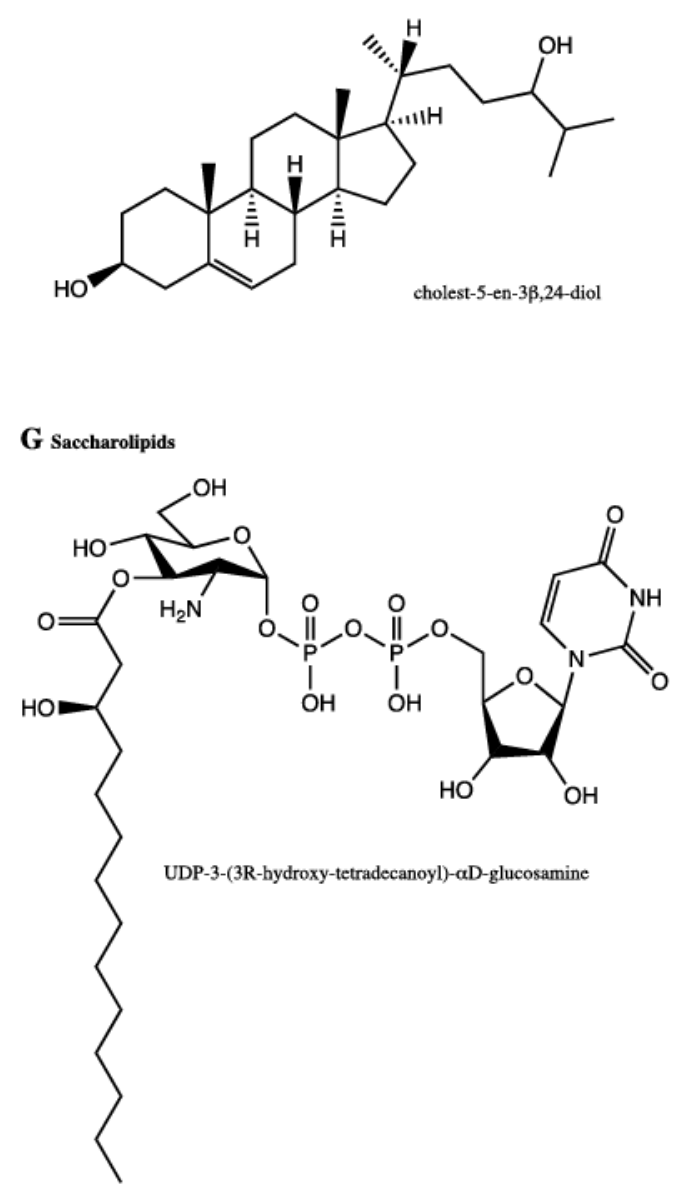

F Prenol lipids<smiles>Cc1c(C)c2c(c(C)c1O)CCC(CCCCCCC[C@H](C)CCCC(C)C)O2</smiles>

Vitamin $\mathrm{E}$

H Polyketides

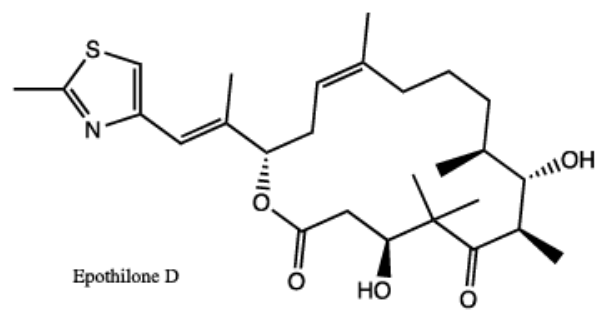

Figure 1.2: Representative structures of the 8 lipid classes. Adapted from Bou Kahalil et al. $^{2}$

\section{Fatty Acyls (Fatty Acids)}

One of the essential lipid classes in the biological system is the fatty acid group. Fatty acids (FAs) are one of the fundamental building molecules for complex lipid species. ${ }^{2}$ Consisting of a terminal carboxylic acid group with either saturated or unsaturated long, unbranched, hydrophobic tail ${ }^{17}$, FAs are highly diverse. ${ }^{2}$ Subclasses of FAs are defined by the degree of branching, the positioning and number of unsaturation (double bonds), the length of the acyl chain, additional functional groups, and the isomer conformation, either cis or trans, in its unsaturation. ${ }^{2,18}$ The structural diversity of an FA 
increases if oxygen, nitrogen, halogen, sulfur heteroatoms, or cyclic structures are also linked to the carbon chains. ${ }^{2}$ Most naturally occurring FAs contain an even number of carbon atoms predominately in an esterified form such as waxes or oils. In plants and bacteria, FAs have been identified that can contain an odd number of carbons, but this is rarely the case in animals. ${ }^{2}$ Figure 1.3 highlights some of the subclasses of fatty acyls.

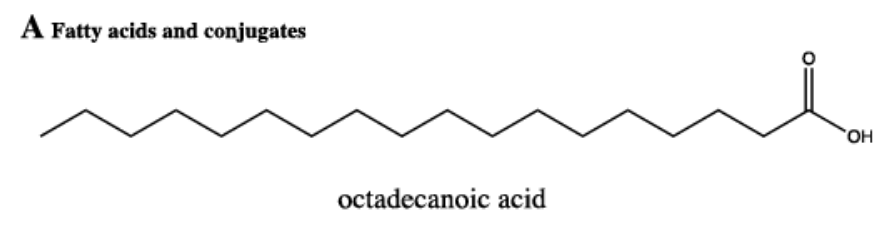

B Fatty alcohols

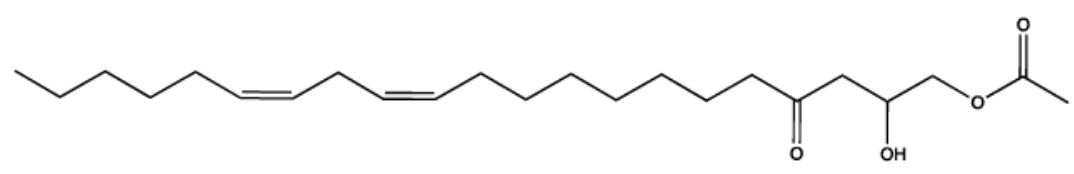

(12Z,15Z)-2-hydroxy-4-oxohenicosa-12,15-dien-1-yl acetate

C Fatty aldehydes

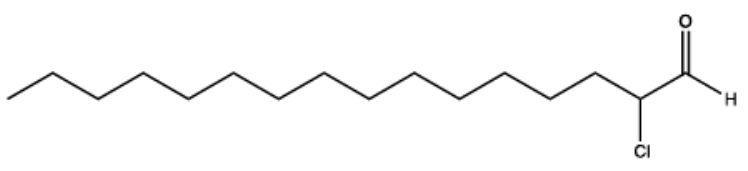

2-chlorohexadecanal

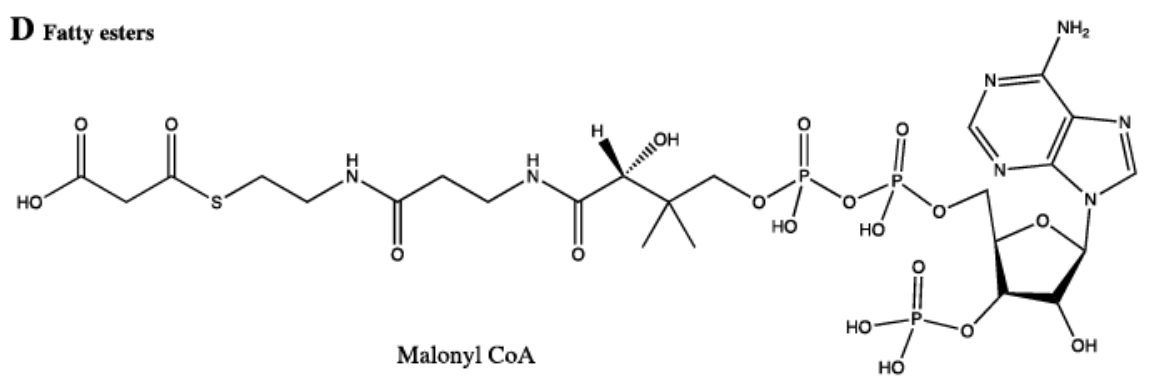

E Fatty amides

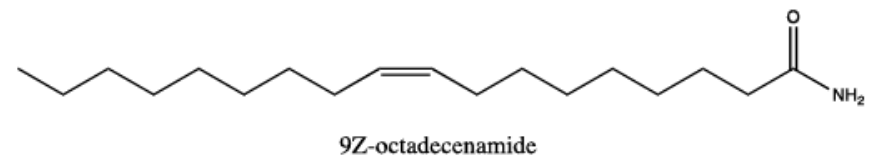

Figure 1.3: Representative structures of 5 fatty acyl classes. Adapted from Bou Kahalil et al. $^{2}$ 


\section{Glycerolipids}

Glycerolipids constitute a class of lipids that consist of a glycerol backbone that are either monosubstituted, disubstituted, or trisubstituted; monoacylglycerols (MGs), diacylglycerols (DGs) and, triacylglycerols (TGs), respectively. ${ }^{15,19}$ The primary role glycerolipids play in biological systems is energy storage. ${ }^{19}$ Glycerolipids are typically found in animal tissues as stored fat ${ }^{1}$, especially the liver ${ }^{20}$. Although there are other subclasses of glycerolipids, such as glyceroglycans, they are beyond the scope of this thesis.

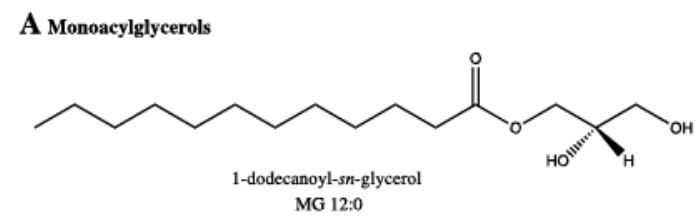

C Alkylacylglycerols
B Diacylglycerols

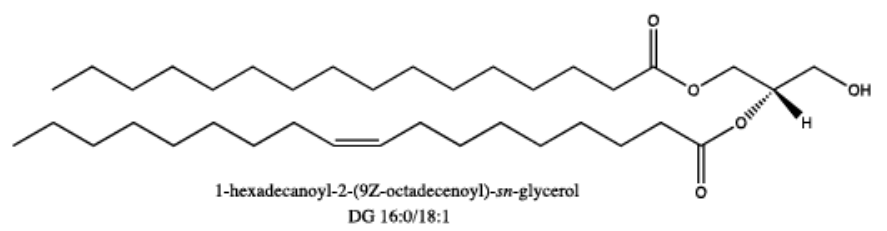

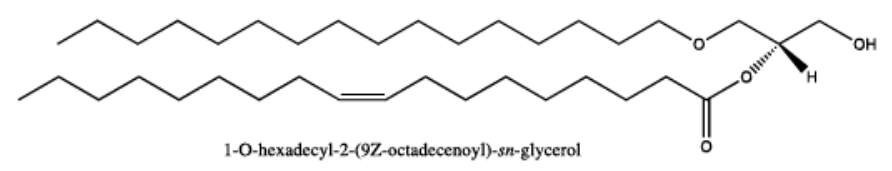

D Alkenylacylglycerols

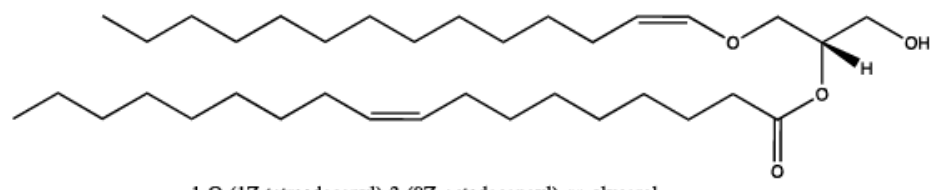

1-O-(1Z-tetraadecenyl)-2-(9Z-octadecenoyl)-sn-glycerol

E Triacylgycerols

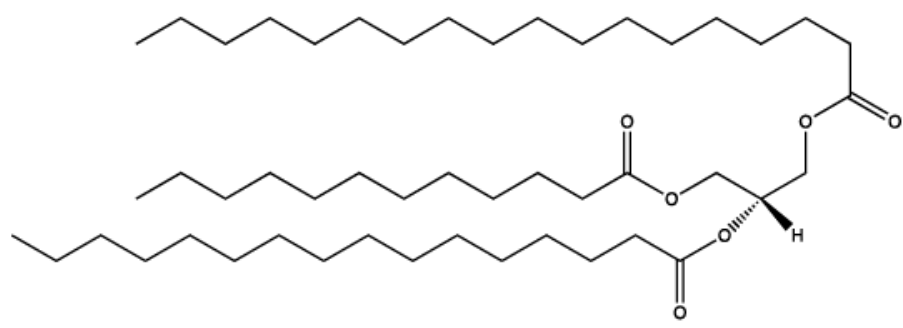

1-O-(1Z-tetraadecenyl)-2-(9Z-octadecenoyl)-sn-glycerol

TG 12:0/16:0/18:0

Figure 1.4: Representative structures of 5 glycerolipid classes. Adapted from Eoin Fahy et al. ${ }^{16}$ 


\section{Sterols}

Sterol lipids, commonly referred to as steroids, are lipids that consist of four fused cyclic ring structures, shown in Figure $1.5 .{ }^{16}$ There are six classes of sterols that are categorized based on their biological functions. These six classes are sterols, steroids, secosteroids, bile acids and derivatives, steroid conjugates, and other sterols. ${ }^{2}$ The most studied sterol lipids in mammalian systems are cholesterol and its derivatives. ${ }^{2}$ Crucial components of the cell membrane, cholesterols play a role in membrane fluidity, where typically more cholesterol in lipid membranes increases membrane fluidity. ${ }^{21}$ Additionally, other sterols like estrogen ${ }^{1}$, testosterone ${ }^{2}$, progesterone ${ }^{1}$ are critical hormones and signalling molecules. ${ }^{22}$ Figure 1.5 illustrates some of the sterol classes.

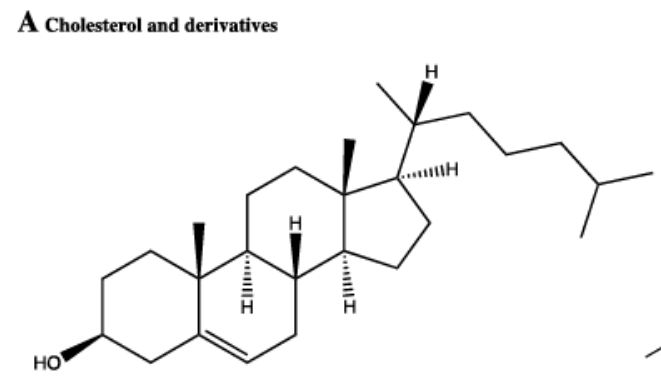

Cholesterol

$\mathrm{C} \mathrm{C}_{21}$ sterols and derivatives

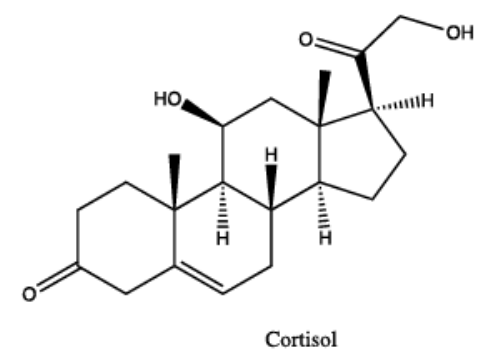

B Cholesteryl esters

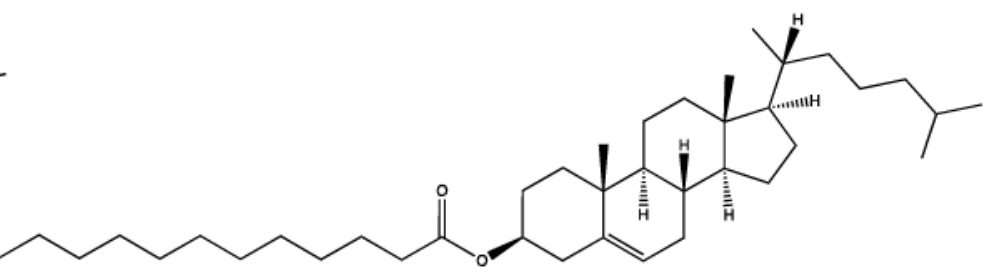

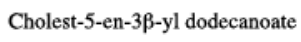

D Secosteroids

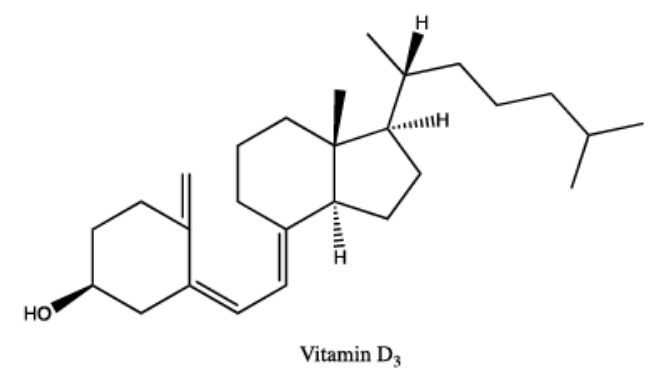

Figure 1.5: Representative structures of 4 sterol classes. Adapted from Eoin Fahy et al. ${ }^{16}$ 


\section{Prenols}

Prenol lipids, consisting of five classes, are essential lipids that are synthesized from the five-carbon unit isopentenyl diphosphate and dimethylallyl diphosphate. ${ }^{2}$ In animals, these units are produced via the mevalonate pathway. Prenol lipids are critical in all cellular organisms since they play an important role as antioxidants ${ }^{23}$ as well as precursors for vitamins $\mathrm{E}^{1}, \mathrm{~K} 2^{24}$, and $\mathrm{A}^{25}$.

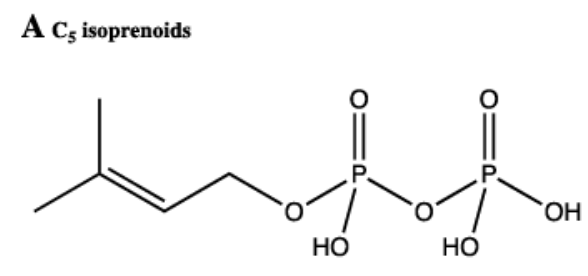

Dimethylallyl pyrophosphate

B $\mathrm{C}_{10}$ isoprenoids

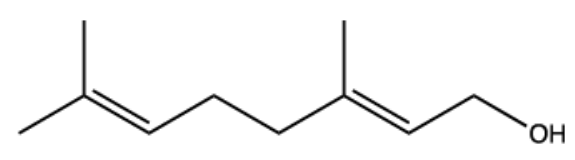

(E)-3,7-dimethylocta-2,6-dienol

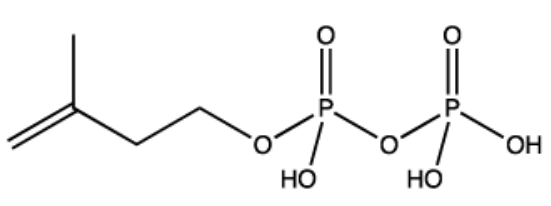

Isopentenyl diphosphate

$\mathrm{C}_{15}$ isoprenoids

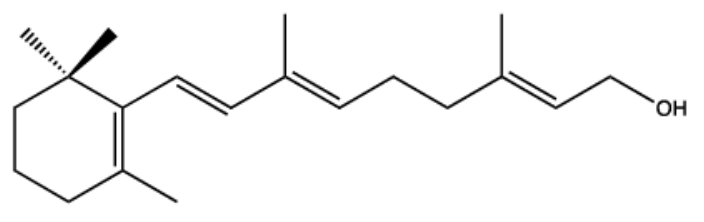

Retinol; vitamin A

D $_{20}$ isoprenoids

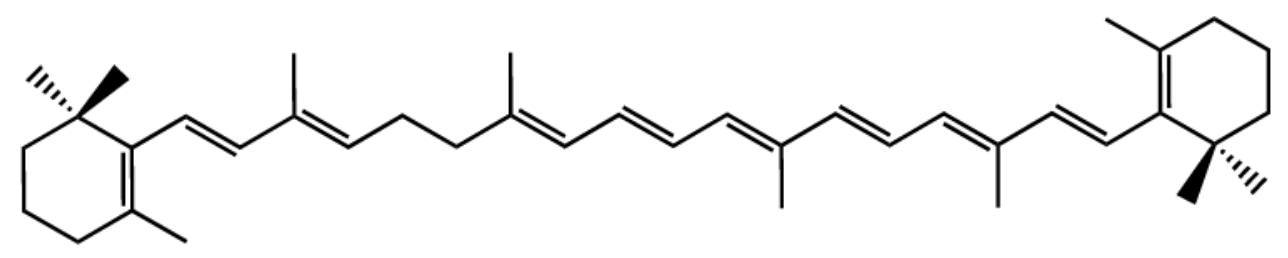

$\beta$-carotene

Figure 1.6: Representative structures of 4 prenol classes. Adapted from Eoin Fahy et al. ${ }^{16}$ Saccharolipids

Saccharolipids are a class of lipids that consist of fatty acids linked directly to a sugar backbone which substitutes the typical glycerol backbone found in glycerolipids and glycerophospholipids. ${ }^{2}$ There are six classes of saccharolipids which are 
acylaminosugars, acylaminosugar glycans, acyltrehaloses, acyltrehalose glycans, other acyl sugars, and other saccharolipids. ${ }^{2,16}$ The most wildly studied saccharolipid is Kdo2lipid A, a component of lipopolysaccharides that are typically found in most gram-negative bacteria. ${ }^{26}$ Some of the saccharolipid classes are illustrated in Figure 1.7. Saccharolipids are not focused on this thesis.

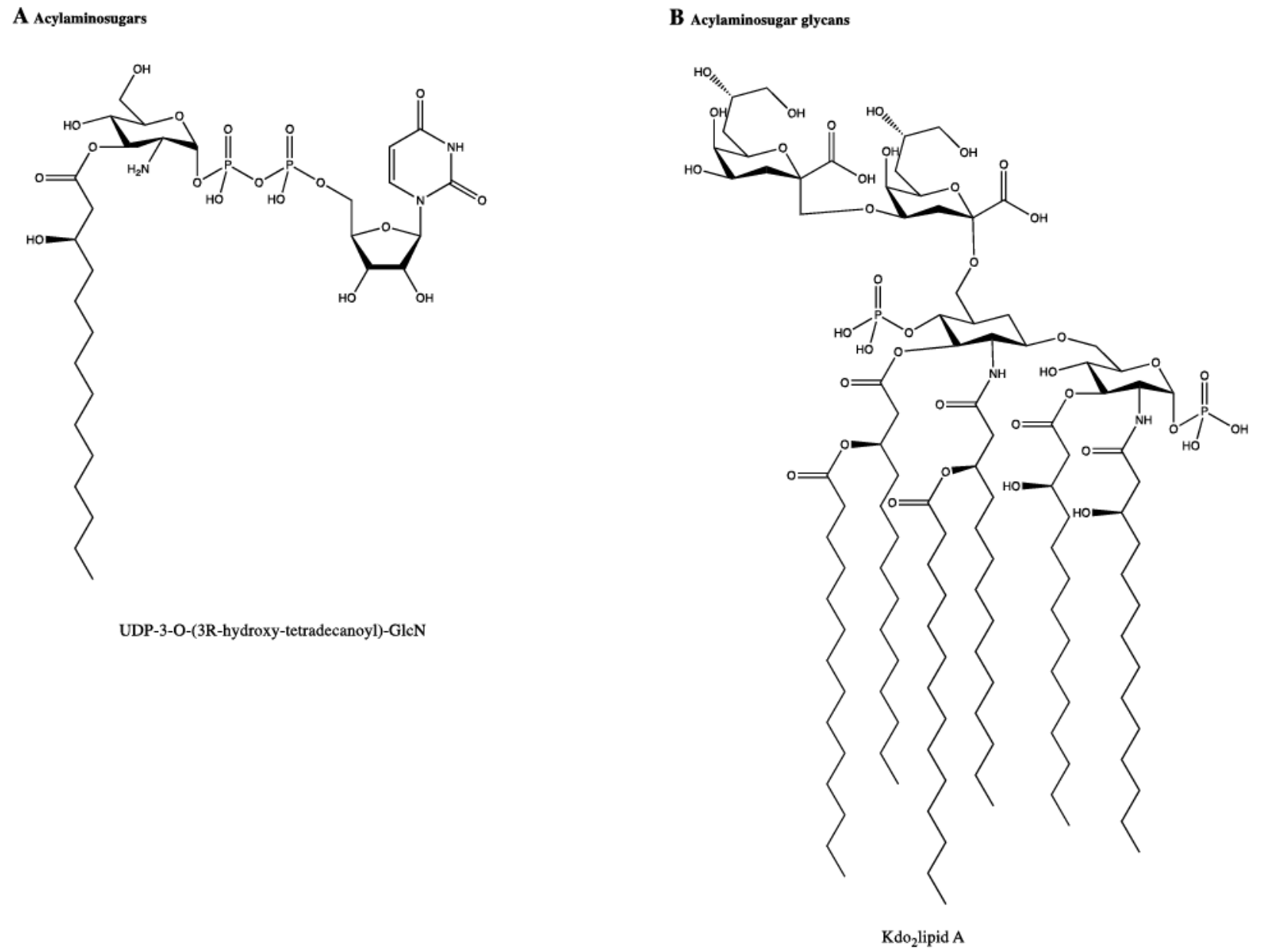

Figure 1.7: Representative structures of 2 saccharolipid classes. Adapted from Eoin Fahy et al. ${ }^{16}$

\section{Polyketides}

Polyketides are a class of highly diverse lipids that are found in microorganisms, plants, and animals. ${ }^{27}$ Synthesized from propionyl-CoA and methylmalonyl-CoA via 
polyketide synthases, analogous to the biosynthesis of fatty acids $^{28}$, these lipids produce many natural product structures ${ }^{2}$ that are used as anti-parasitic, antimicrobial, or anticancer agents. ${ }^{16}$ Its wide diversity is partially due to many modifications to the backbones. These modifications include hydroxylation, glycosylation, methylation, and oxidation. 2,29 A common anti-bacterial drug is erythromycin, which falls under the classification of a polyketide due to its molecular structure, shown in Figure 1.8. ${ }^{16}$

A Macrolide polyketide
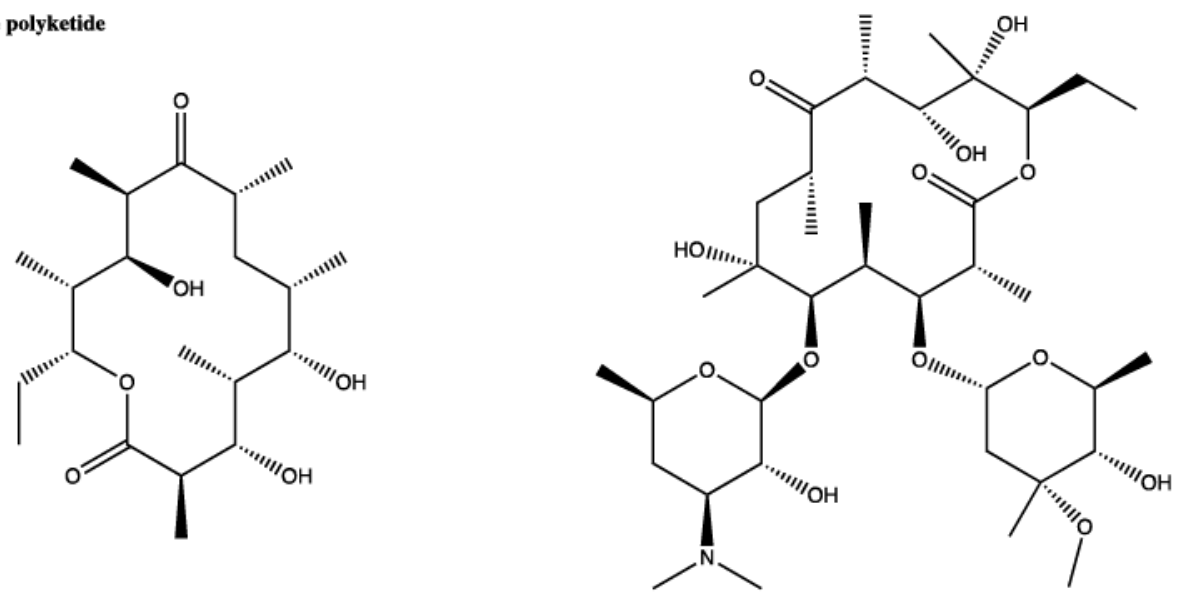

6-deoxyerythronolide B

6-deoxyerythronolide B

B Aromatic polyketide

C Polyketide hybrid

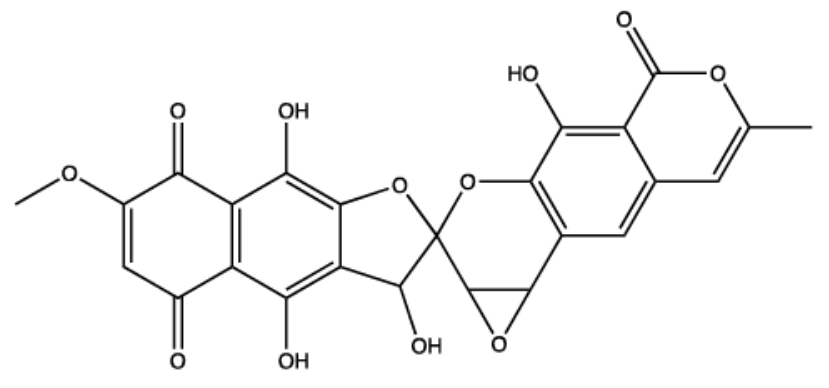

Griseorhodin A

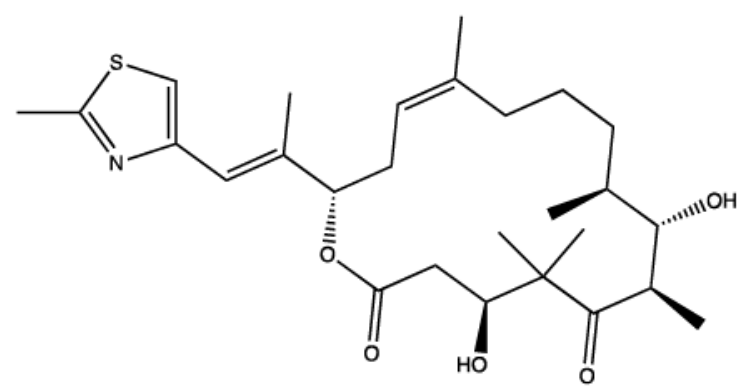

Epothilone D

Figure 1.8: Representative structures of 4 polyketide classes. Adapted from Eoin Fahy et al. ${ }^{16}$ 


\section{Glycerophospholipids}

An essential class of lipids is glycerophospholipids. The cellular membrane is dominantly made up of glycerophospholipids which also serve as binding sites for many cellular and extracellular proteins. ${ }^{2}$ There are well over twenty classes of glycerophospholipids ${ }^{2}$ however, the six most abundant and commonly found in mammalian systems are phosphatidylcholines (PCs), phosphatidylethanolamines (PEs), phosphatidylserines (PS), phosphatidylglycerol (PGs), phosphatidylinositol (PIs) and, phosphatidic acid (PAs). ${ }^{6}$ Glycerophospholipids comprised of a polar head group, composed of a nitrogenous base, a glycerol, or an inositol unit, at the sn-3 position via a phosphodiester linkage. ${ }^{2}$ The $s n-1$ and $s n-2$ position is usually occupied by esterified fatty acyls for glycerophospholipids and a lack of a fatty acyl chain in either the $s n-1$ and $s n-2$ position is usually denoted with a prefix "lyso".2,29 In general, glycerophospholipids are diverse given the variety of substitutes in the polar head group, the acyl chain length, the degree (or amount) of unsaturation (double bonds), and the number of fatty acyl chains it contains, either one or two in either positions or both as illustrated in Figure 1.9. Due to the structural nature of some classes, some lipids are more suited for positive polarity MS analysis such as PC that contains a fixed positive charge, and others are more suited for negative polarity MS analysis such as PS, where a serine group on the polar head results in a net negative charge. ${ }^{16}$ 


\section{A Glycerophosphocholines}<smiles>[R]C(=O)OC[C@@H]([2H])COP(=O)([O-])OCC[N+](C)(C)C</smiles>

1,2-diacyl-sn-glycero-3-phosphocholine

C Glycerophosphoserines<smiles>[R]C(=O)OCCCOP(=O)([O-])OCCC(=O)O</smiles>

1,2-diacyl-sn-glycero-3-phosphoserine

E Glycerophosphoglycerolphosphates<smiles>[2H]C(=O)OC[C@@H]([CH])OCOP(=O)([O-])OC[C@H](O)COP(=O)(O)O</smiles>

1,2-diacyl-sn-glycero-3-phospho-(1'-sn-glycerol-3'-phosphate)

G Glycerophosphoinositol monophosphates

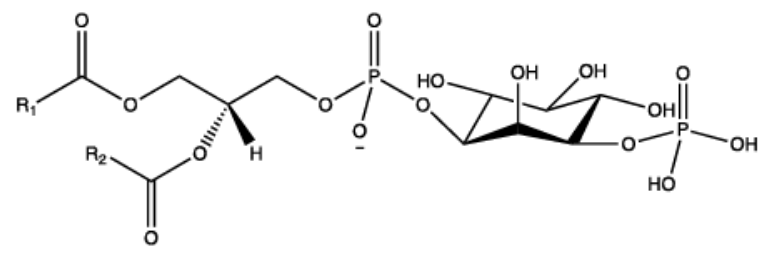

1,2-diacyl-sn-glycero-3-phospho-(1'-myo-inositol-3'-phosphate)

I Glycerophosphoinositol trisphosphates

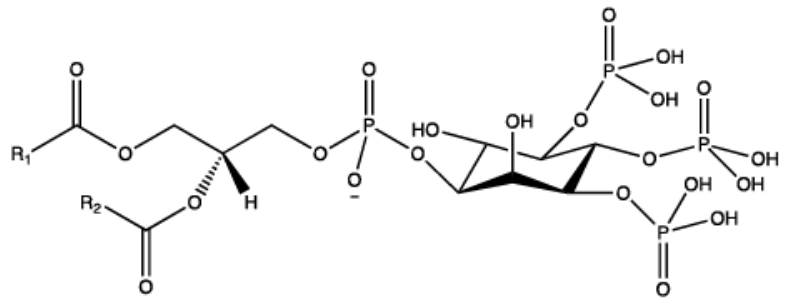

1,2-diacyl-sn-glycero-3-phospho-(1'-myo-inositol-3', 4',5' -trisphosphate)
B Glycerophosphoethanolamines<smiles>[R]C(=O)OC[C@@H]([2H])COP(=O)([O-])OCCN</smiles>

1,2-diacyl-sn-glycero-3-phosphoethanolamine

D Glycerophosphoglycerols<smiles>[R]C(=O)OC[C@@H]([CH])OCOP(=O)([O-])OC[C@H](O)CO</smiles>

1,2-diacyl-sn-glycero-3-phospho-(1'-sn-glycerol)

F Glycerophosphoinositols

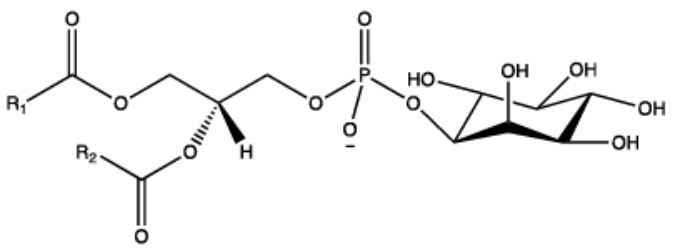

1,2-diacyl-sn-glycero-3-phospho-(1'-myo-inositol)

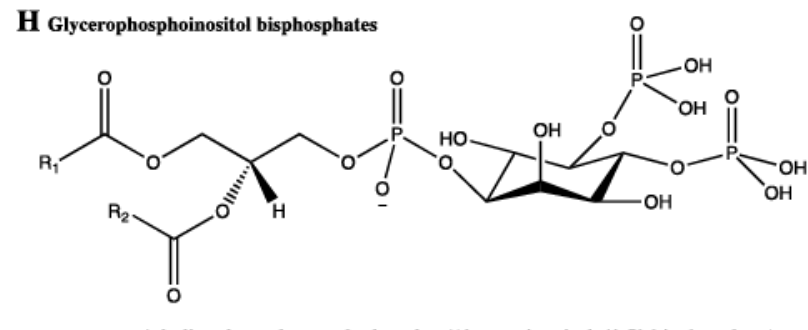

1,2-diacyl-sn-glycero-3-phospho-(1'-myo-inositol-4',5'-bisphosphate)

J Glycerophosphates<smiles>[R]C(=O)OC[C@@H]([2H])COP(=O)(O)O</smiles>

1,2-diacyl-sn-glycero-3-phosphate 
K Glyceropyrophosphates<smiles>[2H]C(=O)OC[C@@H]([2H])COP(=O)(O)OP(=O)(O)O</smiles>

1,2-diacyl-sn-glycero-3-pyrophosphate
L Glycerophosphoglycerophosphoglycerols (cardiolipins)

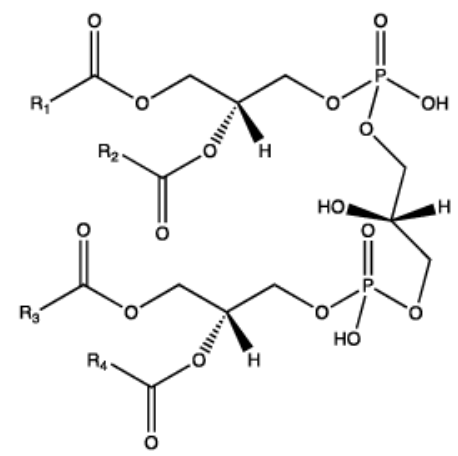

1,3-bis-(1,2-diacyl-sn-glycero-3-phospho)-sn-glycerol

\section{CDP-glycerols}

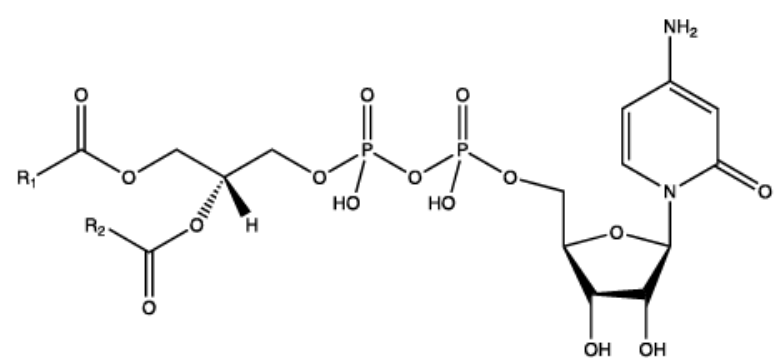

1,2-diacyl-sn-glycero-3-cytidine-5'-diphosphate

O Glycerophosphoinositolglycans<smiles>[R]C(=O)OC[C@@H]([2H])OC[C@]([2H])(O)OP(=O)(O)O[2H]</smiles>

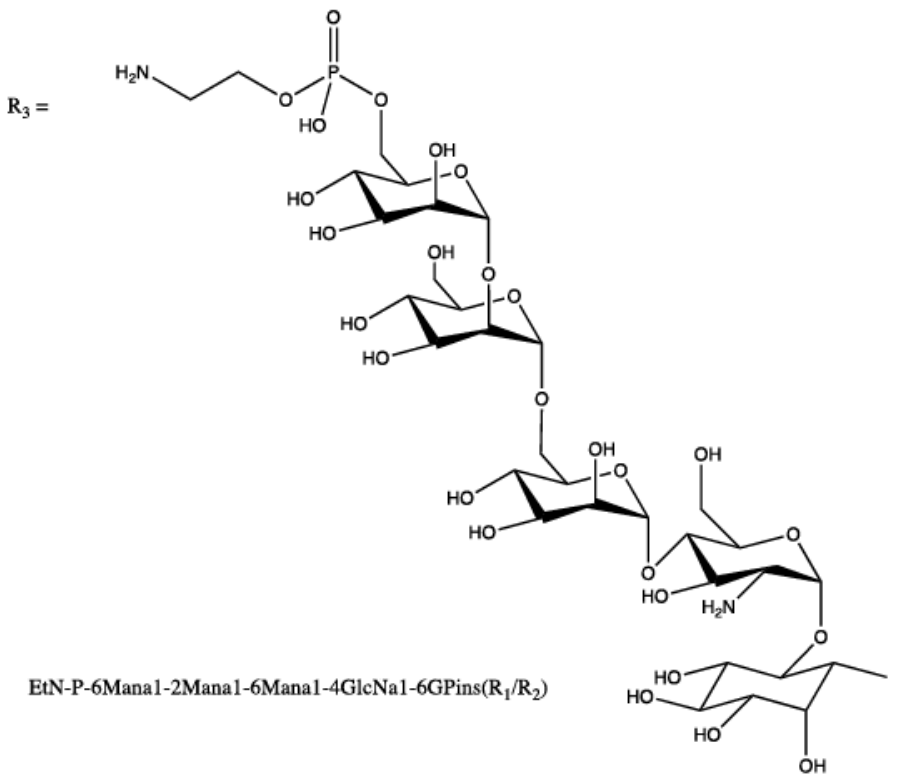

N Glycosylglycerophospholipids

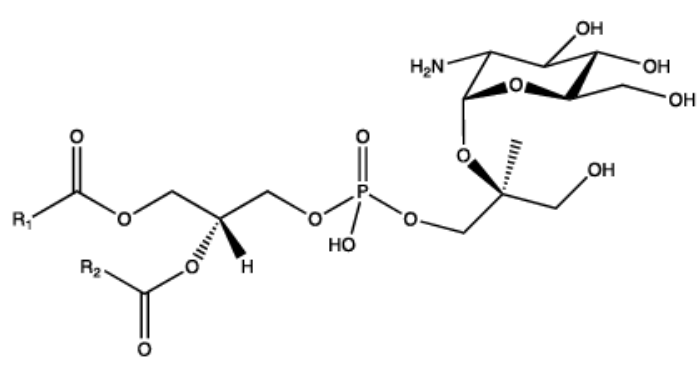

1'-(1,2-diacyl-sn-glycero-3-phospho)-2'- $\alpha$-D-6-glucosaminyl-sn-glycerol

P Glycerophosphonocholines<smiles>[2H]C(=O)OC[C@@H]([2H])OC[C@H](O)OP(=O)([O-])CC[N+](C)(C)C</smiles>

1,2-diacyl-sn-glycero-3-phosphoncholine

Q Glycerophosphonoethanolamines<smiles>[R]C(=O)OC[C@@H]([2H])OC[C@H]([2H])OP(=O)([O-])CCN</smiles>

1,2-diacyl-sn-glycero-3-phosphonoethanolamine 

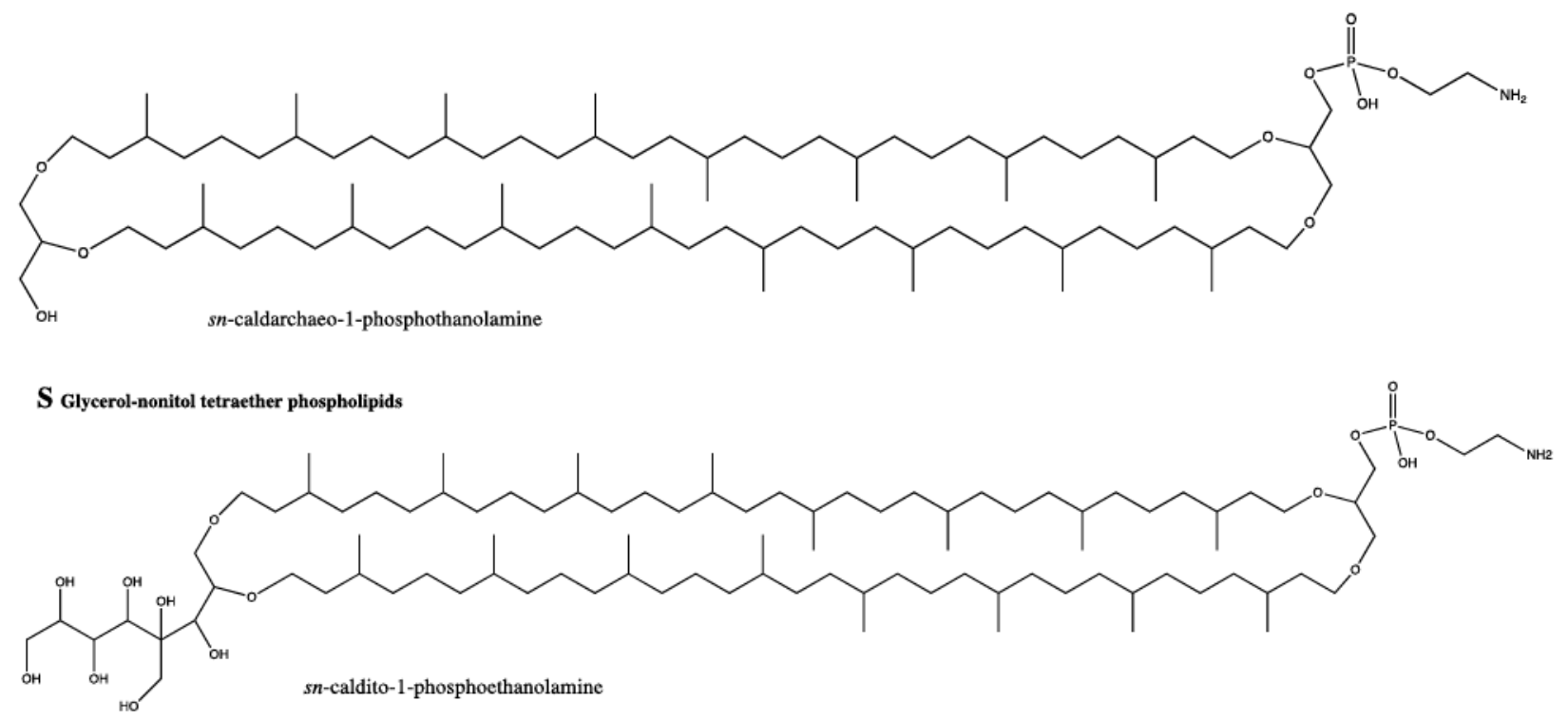

T Other glycerophospholipids

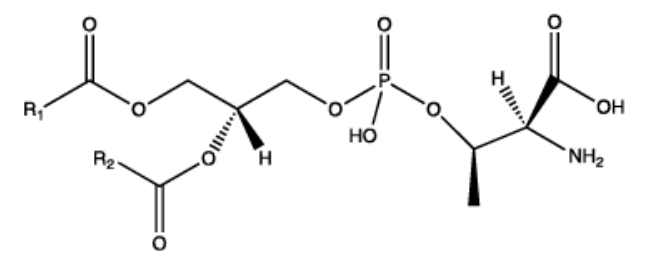

1,2-diacyl-sn-glycero-3-phosphothreonine

Figure 1.9: Representative structures of 20 glycerophospholipid classes. Adapted from Bou Kahalil et al. ${ }^{2}$

\section{Sphingolipids}

Sphingolipids are a unique class of lipids as they are involved in signal transduction and cell recognition. ${ }^{2}$ Unlike other classes of lipids, sphingolipids are typically found in neural tissues. ${ }^{30}$ This class of lipids are derived from the aliphatic amino-alcohol sphingosine or sphing-4-enine, which is synthesized de novo from serine and palmitoylCoA. ${ }^{2,16,31}$ Among the ten classes, the two of interest in this thesis are ceramides (ie. nonhydroxy fatty acid sphingosine ceramides (Cer_NS)) and phosphosphingolipids (ie. sphingomyelins (SM)). SM are phosphosphingolipids that share similar properties to PCs 
as it also contains a choline moiety. ${ }^{16}$ In addition, SM also contains a ceramide moiety. Ceramides consist of a sphingosine moiety, amide-linked to a fatty acyl group. ${ }^{16}$

A Sphingoid bases

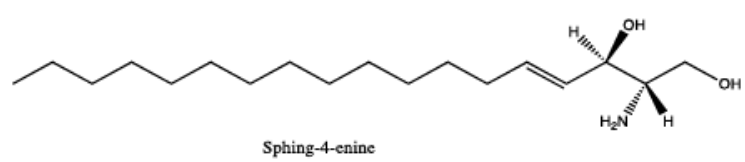

B Ceramides

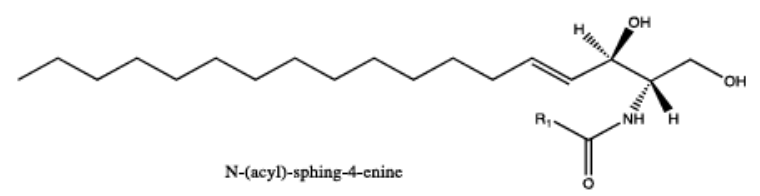

C Phosphosphingolipids

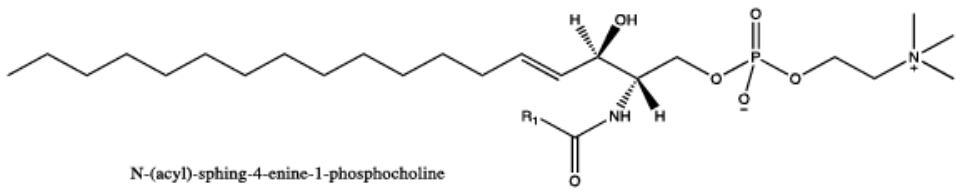

D Phosphonosphingolipids

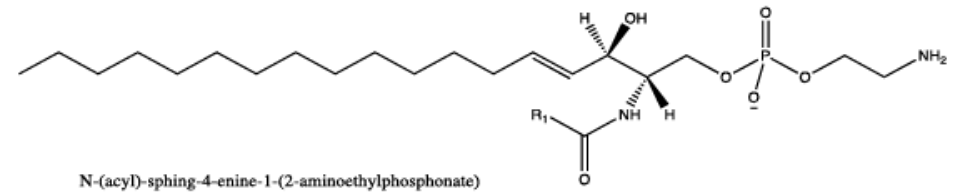

E Neutral glycosphingolipids

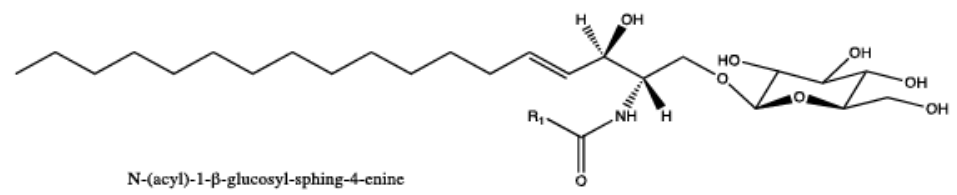

F Acidic glycosphongolipids

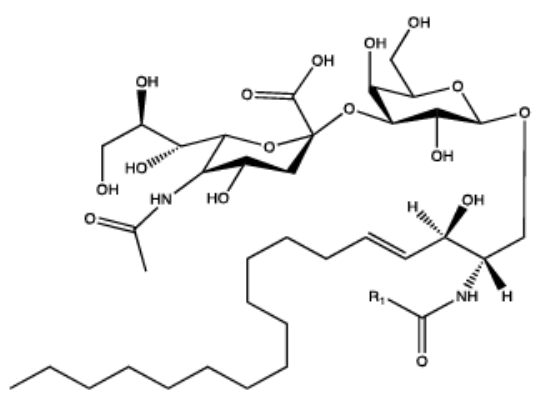

NeuAca2-3Galp-Car
G Basic glycosphongolipids

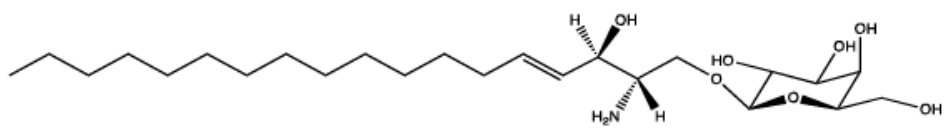

1- $\beta$-galactosyl-sphin-4-enine

H Amphoteric glycosphongolipids

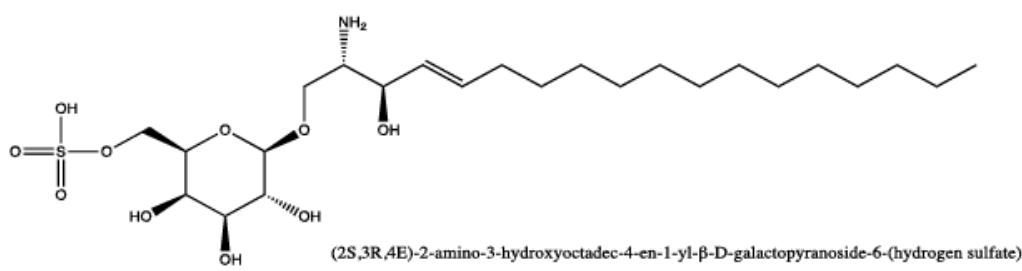

I Other sphingolipids

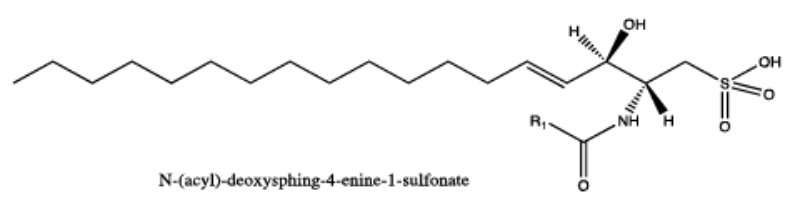

Figure 1.10: Representative structures of the 10 sphingolipid classes. Adapted from Bou Kahalil et al. $^{2}$ 
The fatty acyl group is typically saturated or consist of one double bond (monounsaturated) with an acyl chain length varying between 14 to 26 carbons long ${ }^{2}$ as illustrated in Figure 1.10. SM with a choline moiety is commonly found in mammals while $\mathrm{SM}$ with an ethanolamine headgroup is commonly found in insects. ${ }^{2}$

Frequently, short notations are used as the nomenclature for lipids. An example of this is $\mathrm{PC}(16: 0 / 18: 1)$. This notation describes a phosphatidylcholine with a sn-1 acyl consisting of 16 carbons and 0 double bonds and a sn-2 acyl consisting of 18 carbons and 1 double bond. So far, there are limited methodologies to determine the location of the double bond on the acyl group and far beyond the scope of this thesis. Alternatively, $\mathrm{PC}(16: 0 / 18: 1)$ can be expressed as $\mathrm{PC}(34: 1)$, summing the number of carbons and double bonds in both acyl groups. Identification of the constituent requires technologies that are not available in every laboratory and in this thesis, an algorithmic approach is used to identify lipid species, which is explained below. There are several occasions where lipid species are denoted as PC(16:0_18:1) instead. This indicates that the snpositions are not known whereas the former notation indicates the sn-positions of the fatty acids are proven. ${ }^{32}$

Compared to other "omics" fields, lipidomic is relatively new. Since 2003, the field has been growing rapidly due to new developments in analytical technologies. ${ }^{33}$ With more complex and diverse lipid species being discovered, it is nearly impossible to analyze every single lipid species with one technique. This presents many challenges however, more novel mass spectrometry-based techniques enable these challenges to be overcome by providing better lipidome coverage, accurate identification and, valuable molecular characterization for each individual species within a biological sample. 


\subsubsection{Lipid Extraction and Separation}

To gain insight into the composition, quantitation, and cellular functions of lipids, they must first be extracted from biological samples without degradation and contamination..$^{2,15}$ Many compounds such as carbohydrates, nucleic acids, proteins, and salts found in biological samples must first be separated. Lipids must be extracted with relatively non-polar solvents such as chloroform, benzene, or ethyl ether. ${ }^{2}$ However, lipids that are typically associated with the membrane require polar solvents such as ethanol or methanol to disrupt the hydrogen bonding between lipids and membrane proteins. ${ }^{2}$ In general, all solvents must be freshly distilled and peroxide free to avoid potential oxidation of the unsaturation points. Additionally, de-aeration via nitrogen is critical to prevent/reduce lipid peroxidation. ${ }^{2}$ In general, a biological sample is first homogenized and suspended in an aqueous solution (acetate buffer) and polar/alcoholic solvent such as methanol with a non-polar solvent such as chloroform to form a monophasic system. ${ }^{15}$ Through agitation and centrifugation, an immiscible biphasic system is generated. ${ }^{15}$ The non-polar layer can then be removed and the remaining aqueous/polar lay can be reextracted with a more non-polar solvent. The most common technique used to separate lipids is the Bligh and Dyer extraction which extracts a wide variety of lipids. ${ }^{34}$ Some other techniques include the Folch ${ }^{35}$ and the Matyash ${ }^{36}$ extraction method which utilizes the biphasic system to separate lipids from hydrophilic compounds. ${ }^{37}$ Variations of the Bligh and Dyer extraction technique have been modified to select some lipid classes of interest over others. $^{2}$ 


\subsubsection{Lipid Analysis Before Mass Spectrometry}

Before mass spectrometry, lipids from both biological and synthetic origins are analyzed using different methods. Methods including thin layer chromatography (TLC), colorimetric assays as well as instrumentation methods like nuclear magnetic resonance (NMR), gas chromatography (GC), and liquid chromatography (LC) are used. In this section, a summary of each non-mass spectrometry-based technique will highlight its approaches in lipidomics.

Thin layer chromatography (TLC) is a technique that can be used to rapidly screen, separate, and detect lipids. ${ }^{38,39}$ In TLC, a stationary phase is typically composed of silica on glass, aluminum, or plastic plate. ${ }^{2}$ The mobile phase, a mixture of solvents, varies depending on the lipid classes of interest. ${ }^{39}$ Once the lipid sample is spotted onto the bottom of the silica plate, the mobile phase is allowed to flow up the plate via capillary action ${ }^{40}$ that separates the lipids depending on size and polarity. Once complete, iodine vapour or class-specific dyes are employed for detection. ${ }^{5}$ Although TLC is a rapid method to screen for lipids, there are many disadvantages. Notably, TLC lacks specificity and resolution. Additionally, prolonged exposure to air can lead to lipid degradation or oxidation, further limiting lipid identification. ${ }^{2}$

Colorimetric assays are a technique used to quantify total lipid content in a biological sample..$^{41,42}$ Generally, spectrophotometry and spectrofluorimetry use a source of light that determines the quantity of analyte based on the amount of light absorbed or emitted, respectively, in its detector ${ }^{40}$ In spectrophotometry, a specific dye that reacts with lipids is employed to alter colouration. This changes the amount of light able to pass through the sample and into the detector. ${ }^{43}$ In spectrofluorimetry, a fluorescent dye is 
used to determine specific lipid species. The light emitted determines the quantity/concentration of the lipid of interest. ${ }^{44}$ While colorimetric assays are informative in quantifying lipid content, they are not accurate since other cellular components such as proteins or nucleic acids often interfere or skew results. ${ }^{42}$ Additionally, colorimetric assays provide no information in the lipid class nor species present in the sample.

Gas chromatography (GC) is a technique that separates analytes from a mixture based on the analyte's affinity to both the stationary phase and the mobile phase. GC itself is only compatible with volatile compounds that can separate under high temperatures. This unfortunately does not work for many biological molecules as many would degrade during chromatographic separation. ${ }^{2}$ In lipidomics, utilizing GC is highly problematic. Many lipid classes such as glycerolipids and glycerophospholipids are not compatible since the extreme heat would degrade them into their glycerol and fatty acid moieties ${ }^{45}$ Other lipid classes like fatty acids and some sterols are effective with GC. Derivatization of lipid species can improve volatility however, sample recovery of low abundant lipids as well as large sample amounts required. ${ }^{2}$ Although GC is sensitive, lipids may experience isomerization and/or decomposition when under such high temperature. ${ }^{2}$

High-performance liquid chromatography (HPLC) as an alternative separation technique compared to $\mathrm{GC}$ is more favorable in lipidomics. This is because glycerophospholipids, sterols, fatty acids, neutral lipids, and many other classes can separate effectively ${ }^{2}$ without the worry of decomposition from high temperature exposure ${ }^{45}$ while maintaining high sensitivity. Additionally, HPLC is more versatile since both normal-phase and reversed-phase HPLC can be used. In normal-phase HPLC, 
glycerophospholipids are separated based on the polarity of the head group. In reversedphase HPLC, phospholipids are separated based on the hydrophobicity of the FA chain(s). ${ }^{2}$ Post column separation allows many different techniques such as ultraviolet, flame ionization, fluorescence, or mass spectrometry to directly analyze the eluted lipids. ${ }^{2}$ These techniques, especially mass spectrometry, have significantly enhanced the ability to accurately identify and quantify lipid species from a biological sample.

\subsection{Mass Spectrometry}

Mass spectrometry (MS) is a powerful and versatile instrumental technique that has revolutionized the analytical fields within many disciplines such as chemistry, medicine ${ }^{46}$, and forensics ${ }^{47} .{ }^{48}$ Lipidomics is no exception. Advancements in MS provide in-depth insight into the composition of the lipidome given a biological sample. ${ }^{2} \mathrm{MS}$ was first invented by Sir J.J. Thomson who demonstrated this technique by separating isotopes of neon gas $\left({ }^{20} \mathrm{Ne}\right.$ and $\left.{ }^{22} \mathrm{Ne}\right)$ by their mass-to-charge ratio $(\mathrm{m} / \mathrm{z}){ }^{49}$ The appropriate unit to express mass-to-charge ratio is Thomson (Th). Since then, mass spectrometry has advanced significantly but the three fundamental components remained the same. An ionization source, a mass analyzer, and a detector, shown in Figure 1.11, are the key components to produce a mass spectrum.

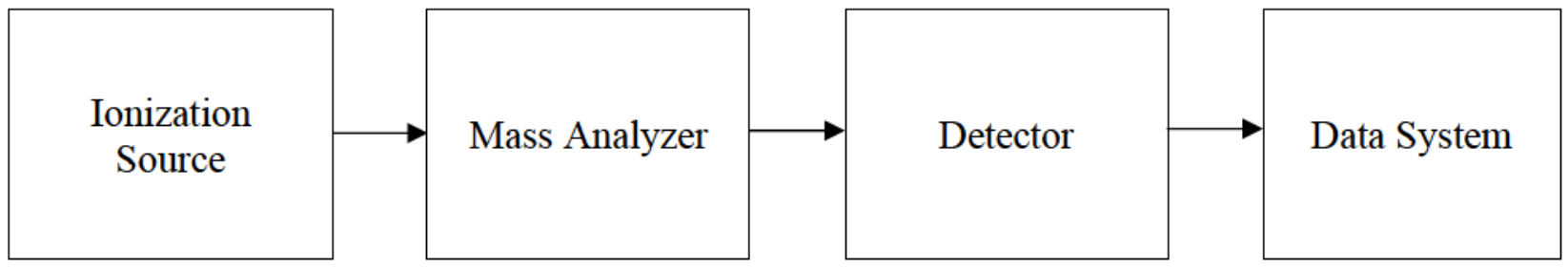

Figure 1.11: Fundamental components of a mass spectrometer.

Before analysis of samples can begin, analytes must first be ionized in a gaseous state. Since not all analytes are charged nor in a gaseous state, an ionization source is 
employed to produce analytes with an overall positive or negative charge, while simultaneously vapourizing the analyte into the gas phase. ${ }^{50}$ There are a variety of ionization techniques available depending on the type of sample or class of molecules. This can be classified into two groups, hard ionization, and soft ionization. ${ }^{51}$ Hard ionization techniques invoke a large degree of fragmentation of the parent ion using harsh methods (high energy) to induce a charge on a molecule. ${ }^{52}$ Some examples of hard ionization techniques include chemical ionization (Cl) and electron ionization (EI) for small, organic compounds, and atmospheric pressure chemical ionization (APCl) for small, moderately polar biomolecules. With soft ionization techniques, fields of study such as proteomics, genomics, metabolomics, and lipidomics are possible with MS since these techniques ionize large biomolecules without obliterating their molecular structure to produce ions ${ }^{53}$ Common examples of soft ionization techniques include matrix-assisted laser desorption ionization (MALDI) and electrospray ionization (ESI) ${ }^{54}$, which the latter will be focused on in Chapter 1.2.1. While all these techniques differ in methodology, all produce ions in a gaseous state for mass spectrometry.

After ionization, a mass analyzer is employed to determine the $\mathrm{m} / \mathrm{z}$ of the ions based on their interaction with the electric or magnetic field. ${ }^{50}$ These ions can be separated through many different analyzers such as a quadrupole, where mass is filtered, an ion trap, where ions are separated in space, or in a time-of-flight (TOF) tube, where ions are separated via time.$^{55}$ Additionally, hybrid instruments combine different types of mass analyzers for a variety of experiments. Examples include hybrid triple quadrupoles linear ion traps, hybrid linear ion trap/orbitrap, and hybrid quadrupole time-of-flight (QTOF). Specific mass analyzers and the hybrid mass spectrometer used in this thesis 
will be discussed in the later chapters. While there are many mass analyzers available, each has variable mass range, resolution, and sensitivity.

Once ions have been separated, a detector is required to convert the separated ions into an electric signal that gets recorded as a mass spectrum. The mass-separated ions collide onto a surface that induces a quantifiable signal based on the number of collisions.$^{56}$ There are many types of commercially available detectors such as a channel electron multiple (CEM) for hybrid triple quadrupole-linear ion trap and a chevron microchannel plate (MCP) for hybrid QTOF-MS. However, since the only mass spectrometer used in this thesis is a hybrid QTOF-MS (see Chapter 1.2.5), the latter ion detector will be briefly discussed in Chapter 1.2.4.

\subsubsection{Electrospray lonization (ESI)}

Electrospray ionization (ESI), mentioned previously, is a soft ionization technique ${ }^{52}$ that is commonly used for large, non-volatile biomolecules. ${ }^{53}$ Following separation, typically from HPLC, the sample is sprayed into the MS at the end of a highly charged capillary needle, forming charged droplets. ${ }^{2}$ The entirety of the work presented in this thesis is performed using a positive polarity, therefore, any aforementioned term of charged ions will be equivalent to positive ions. The spray that is emitted from the capillary produces a plume of charged droplets formally known as a Taylor cone. A conventional ESI tip introduces the sample at a flow rate between $4-200 \mu \mathrm{L} / \mathrm{min} .{ }^{57}$ The formation of small, charged droplets is due to the high electric potential, typically between 2-6 kV. ${ }^{50}$ Desolvation occurs where solvated ions are concentrated until ESI yields evaporated ions. ${ }^{40}$ These ions are pulled towards the mass spectrometer via charged metal plates 
where most ions are reduced; however, a small amount can enter the orifice and into the mass analyzer. ${ }^{50} \mathrm{~A}$ schematic of ESI is shown in Figure 1.12.

Inlet to mass spectrometer

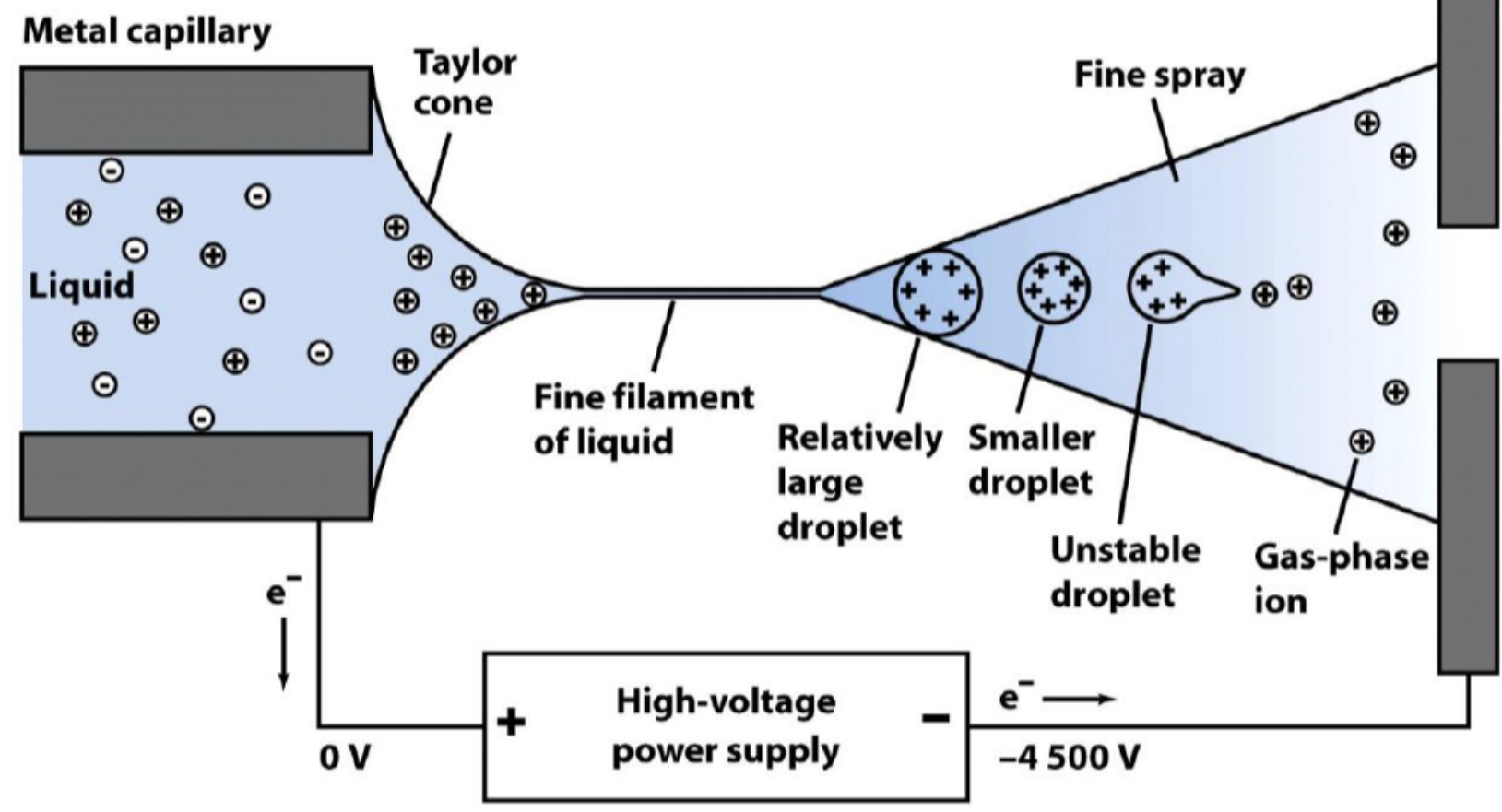

Figure 1.12: Schematic of electrospray ionization. Figure adapted from Harris, D. C. $9^{\text {th }}$ Ed. ${ }^{40}$

\subsubsection{Quadrupole Mass Analyzer}

A quadrupole consists of four parallel, hyperbolic metal rods that are connected electronically. ${ }^{40}$ The trajectory of the ions is dictated by the potential that is applied to those rods, illustrated in Figure 1.13. An alternating current $(A C)$ is applied at a controllable radio frequency (RF) that rapidly alternates. ${ }^{58} \mathrm{~A}$ positive potential is briefly applied to two rods that are opposite of each other while a negative potential is briefly applied to the other opposing rods. These polarities rapidly cycle during the experiment ${ }^{40}$ which results in the ion's spiraling path. To select for a specific $\mathrm{m} / \mathrm{z}$, a direct current (DC) potential is applied. ${ }^{58}$ When DC is applied, this causes an increase in amplitude which 
results in the change of the ion's motion along the $x-y$ plane. If the ion resonates the same frequency in the $x-z$ and $y-z$ planes, the ion is stable in the $x-y$ plane, allowing transmission of the ion to the detector ${ }^{58}$ Briefly, increasing the electric field in the $x-z$ plane such that the overall potential is positive, and the DC field is stronger than the RF field, heavier ions are focused to the center axis of the rods. This allows transmission of heavier ions to the detector while destabilizing lighter ions which results in a collision with the quadrupole, neutralizing them. The opposite occurs when selecting lighter ions. If the DC field is weaker than the RF field, ie. an overall negative potential is applied in the $y-z$ plane, which destabilizes heavier ions resulting in a collision with the quadrupole, neutralizing them. An RF only mode is also used to allow transmission of many ions to the detector. This mode allows all resonating ions to produce a full mass scan. ${ }^{59}$

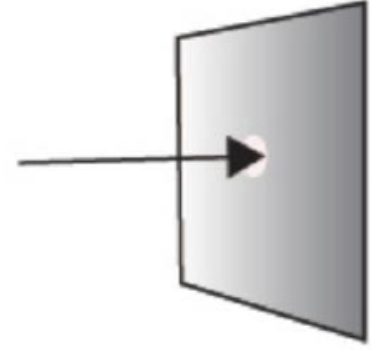

Ion Source

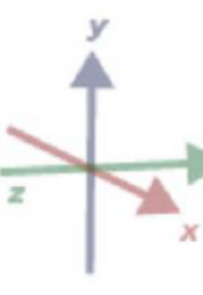
.

.


velocities depending on the $\mathrm{m} / \mathrm{z}^{40}$ This allows lighter ions to travel faster, thus reaching the detector earlier than heavier ions which travel slower than the former. Figure 1.14 illustrates a simple schematic of a TOF where ions travel in the drift tube. In a TOF, the drift region allows separation via "time" of heavier and lighter ions. The longer the TOF tube, the longer time the ions will spend travelling which results in better separation and increases resolution. ${ }^{58}$ To increase the drift time without increasing the length of the drift tube, TOF instruments consist of a reflectron ion mirror. ${ }^{40} \mathrm{~A}$ reflectron ion mirror consists of many electrode rings that produce increasingly stronger electromagnetic fields which change the trajectory of the ions, effectively doubling the length of the time-of-flight path ${ }^{40}$ while correcting for slight variances in arrival time for ions with identical $\mathrm{m} / \mathrm{z}^{62,58}$ Smaller ions will not travel as deep into the reflectron compared to larger ions.

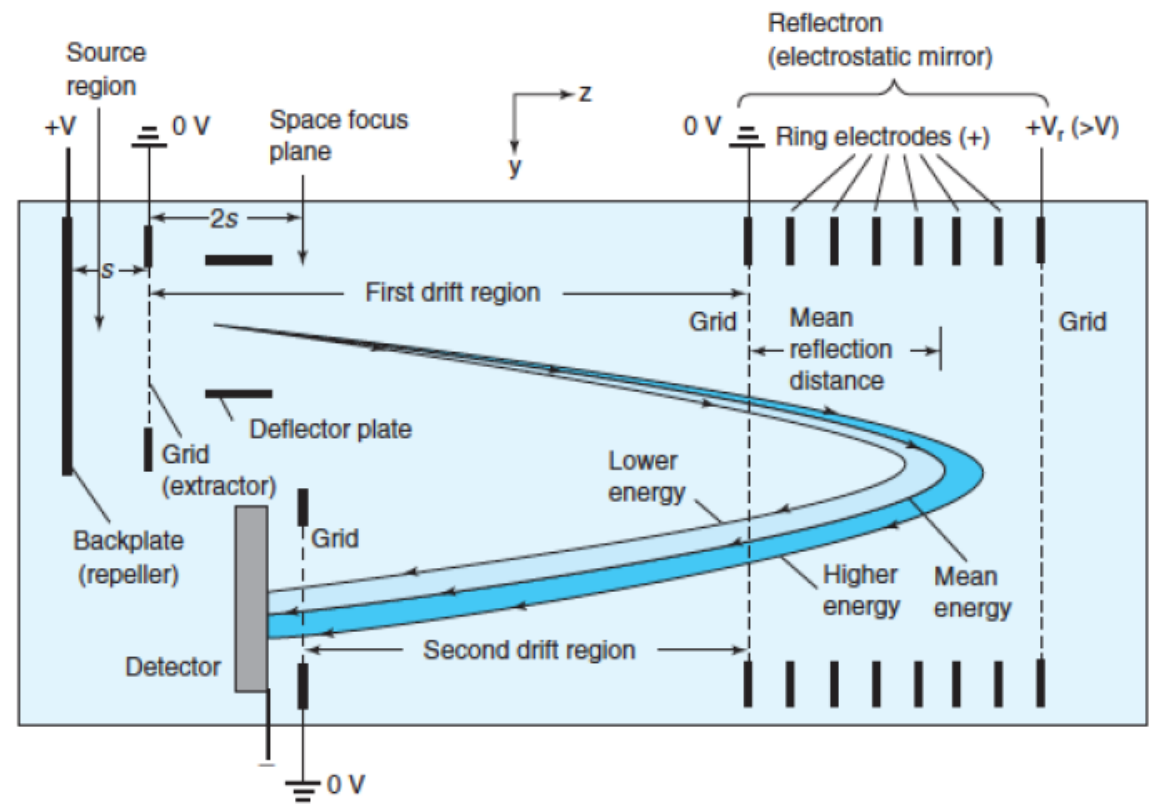

Figure 1.14: Schematic of a TOF. Figure adapted from Harris, D. C. $9^{\text {th }}$ Ed. ${ }^{40}$

While linear TOF exists, it only works best if the initial kinetic energy is constant, the initial direction is the same, and the spatial distribution of the ions in the accelerator 
is the same. However, all these conditions are not always met. Examples of these conditions not being met are illustrated in Figure 1.15. To accelerate ions uniformly, a TOF-MS with an orthogonal ion accelerator was designed. A TOF with an orthogonal ion accelerator has the drift tube perpendicular to the direction of the ionization source. This increases resolving power ${ }^{63}$ as well as ensuring that ions with the same $\mathrm{m} / \mathrm{z}$ will hit the detector at the same time. ${ }^{6}$ The reflectron remains located at the end of the drift tube. This is highly important since the reflection corrects for any slight variances for ions with identical $\mathrm{m} / \mathrm{z}^{40}$ For example, if ions of identical $\mathrm{m} / \mathrm{z}$ start with different spatial distribution in the ion accelerator, the ions that are accelerated faster will penetrate deeper into the reflectron compared to ions with slower acceleration. Since the ion is in a "deeper" region of the reflectron, a larger repulsive energy is experienced to reaccelerate the ion towards the detector. Figure 1.16 illustrates clearly how a reflectron corrects for this variation for ions of the same $\mathrm{m} / \mathrm{z}$ to reach the detector at the same time. Unlike a quadrupole mass analyzer, TOF is incapable of mass filtering. Additionally, TOF mass analyzers do not have a theoretical mass limit which makes analysis of small and large biomolecules possible. ${ }^{58}$ 


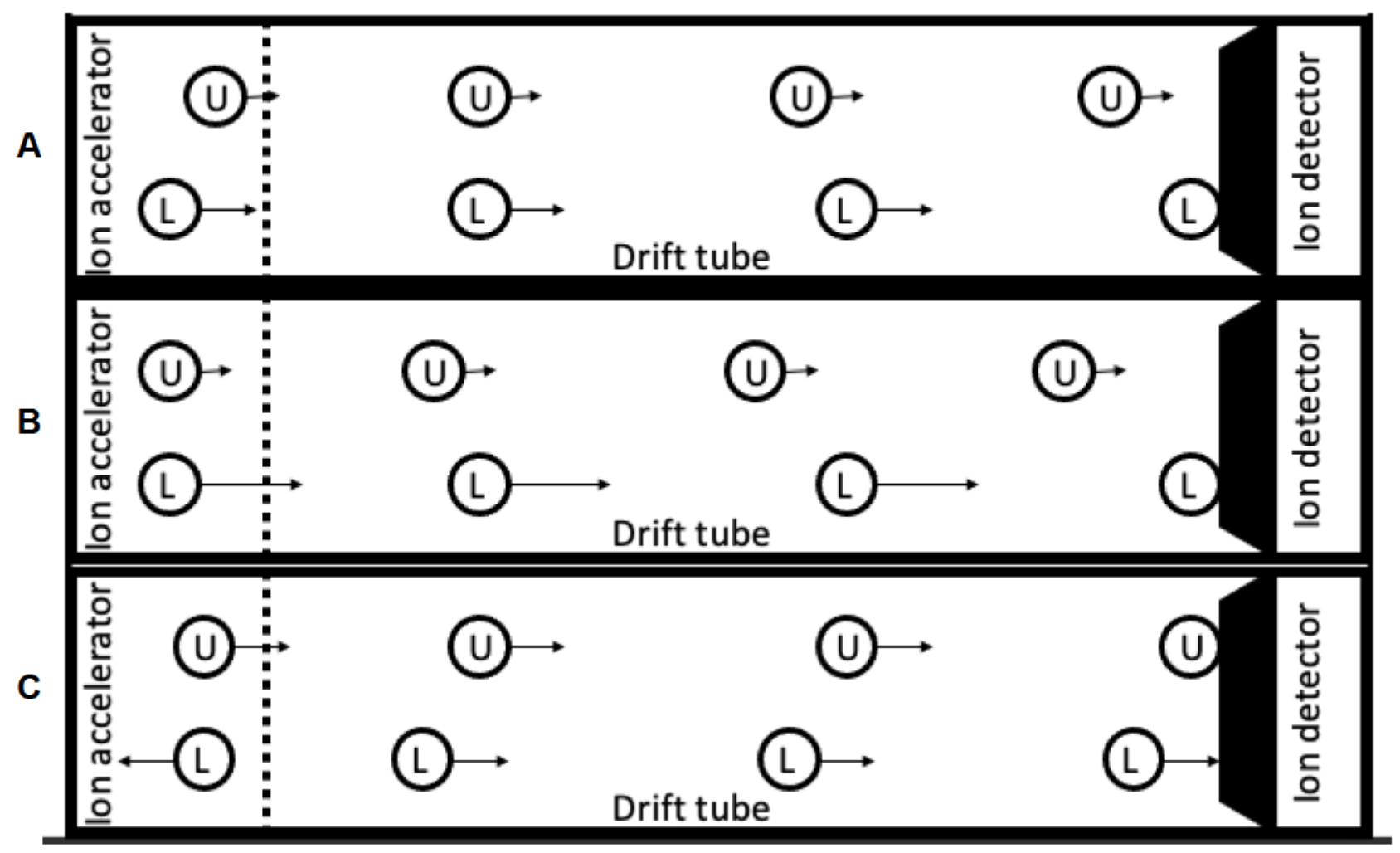

Figure 1.15: Different non-uniform conditions that decreases resolution in a TOF-MS. The $U$ and $L$ designates two ions with identical $\mathrm{m} / \mathrm{z}$. A) Not starting at the same point in the ion accelerator, resulting in different velocities in the drift tube. This results in the $U$ ion to hit the detector later than $L$. B) lon $L$ starts off with more residual velocity than $U$, resulting in $L$ hitting the detector earlier than ion $U$. C) Pulsing of the ion accelerator causes ion $L$ to travel the other way. This results in ion $U$ hitting the detector earlier. Figure adapted from Smith. ${ }^{64}$ 


\section{"Reflectron" ion optics:}

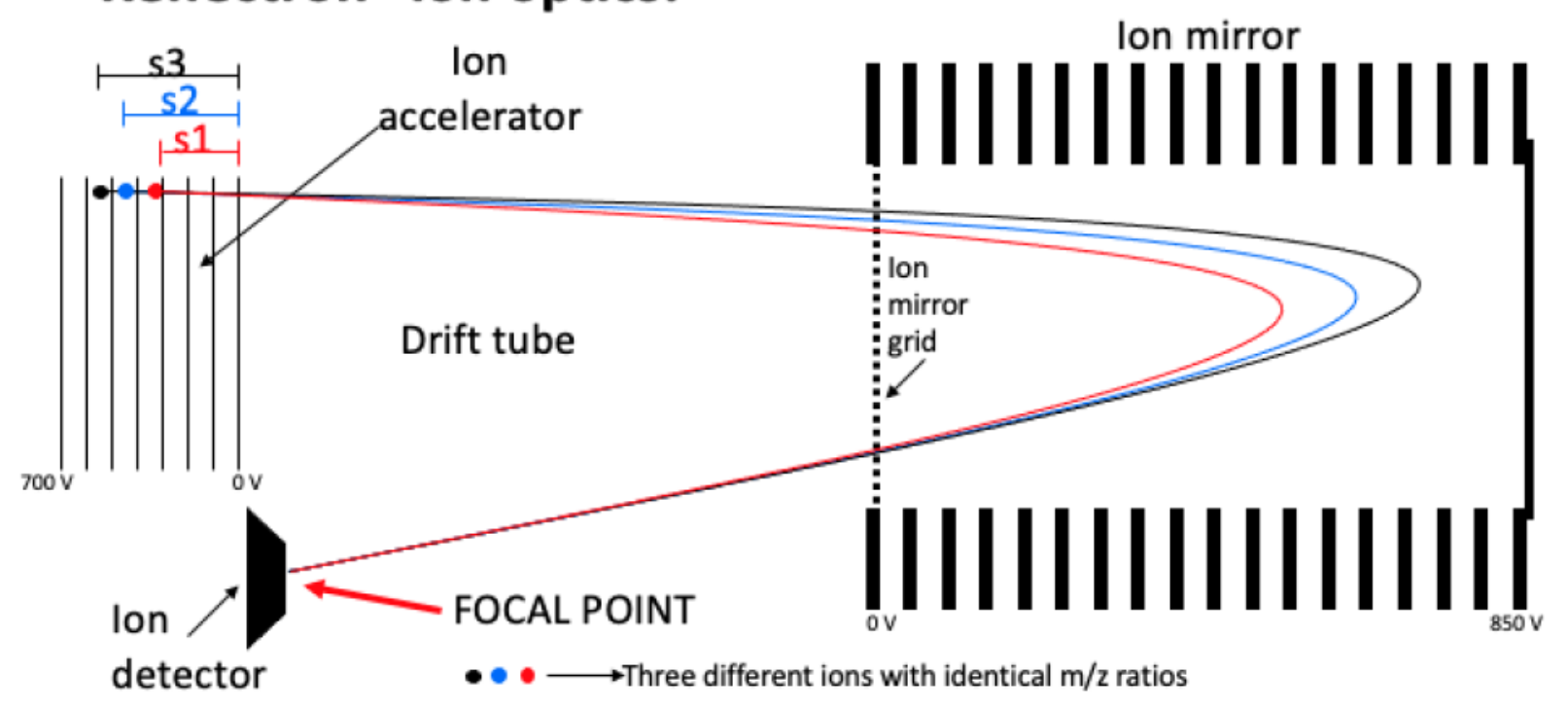

Figure 1.16: Schematic of how a reflectron or ion mirror corrects for the different spatial distribution with identical $\mathrm{m} / \mathrm{z}$ ions. Figure adapted from Smith. ${ }^{64}$

\subsubsection{Ion Detector: Multichannel Plate Detector}

Briefly discussed in Chapter 1.2, a detector in mass spectrometry converts the separated ions into an electric signal that gets recorded as a mass spectrum. The ions collide onto the detector's surface that induces a quantifiable signal that is proportional to the number of collisions. ${ }^{56}$ The detector used in this work was the microchannel plate (MCP) detector. Since only a small fraction of the ions can reach the detector, the detector itself must be very sensitive. The detector is composed of two microchannel plates with angled channel electron multipliers (CEM) that are configured in a shallow v-like shape (chevron). ${ }^{65}$ As illustrated in Figures 1.17 and 1.18, this maximizes the signal that is generated from a single ion. As an ion collides with the first MCP, it results in an electron emission that cascades with every additional collision as more electrons are emitted down the channels in the first MCP. As the electrons exit the first MCP, an electric field is applied and these electrons accelerate while spreading out, colliding in multiple CEM channels in 
the second MCP. Once the cascade of electrons exits the second MCP, they collide with a metal anode in which the amplified signal is recorded. ${ }^{40,65}$

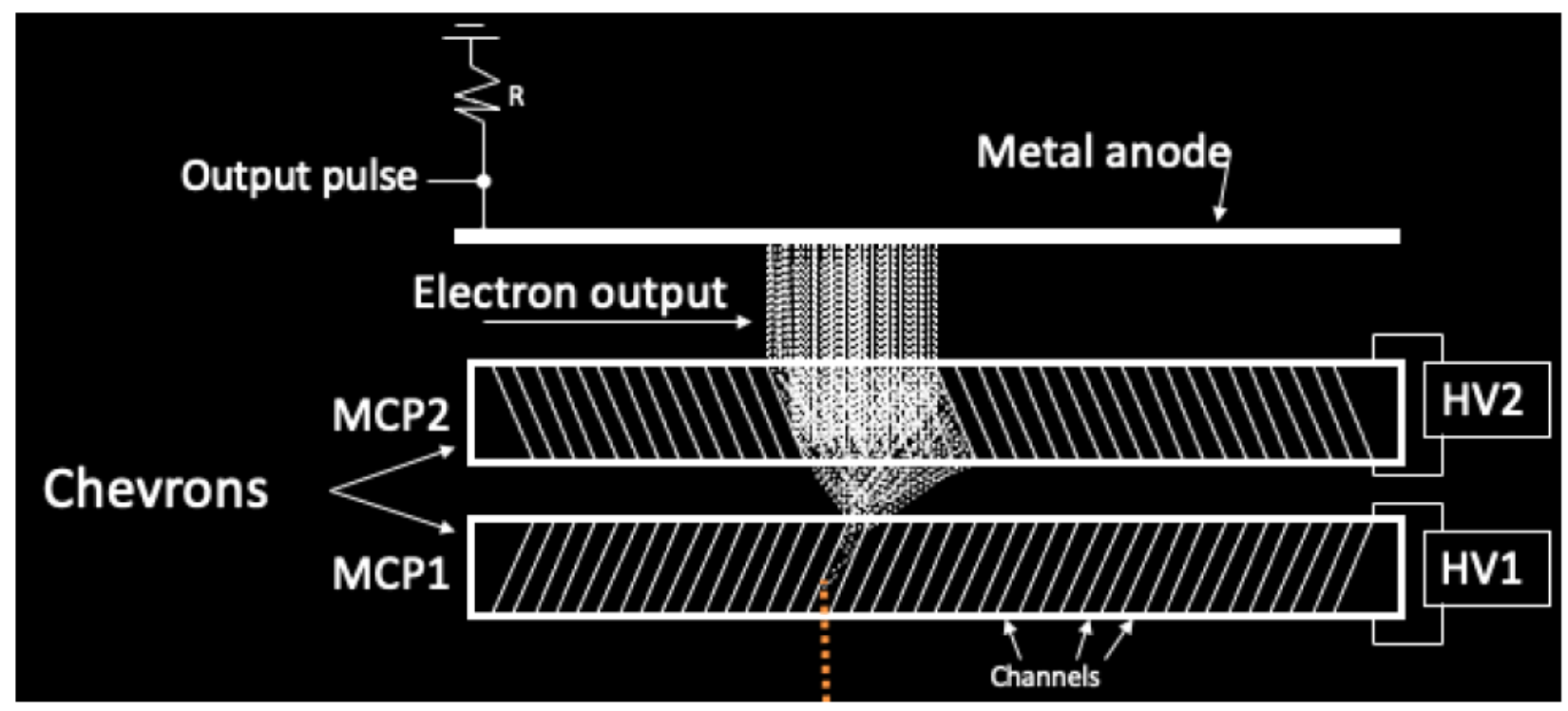

Figure 1.17: Scheme of a multichannel plate detector. Adapted from Smith. ${ }^{64}$
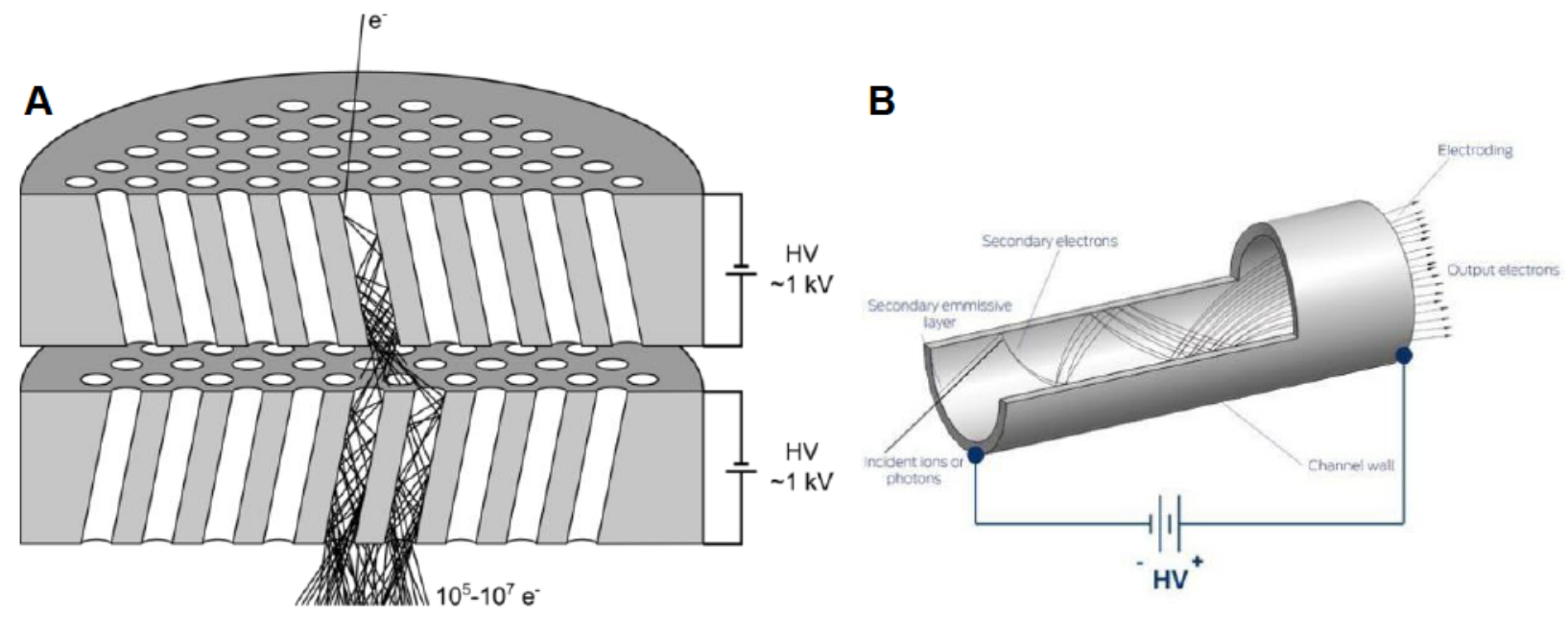

Figure 1.18: A) Scheme of two microchannel plates and a cascade of electrons from one electron emission. Adapted from Eberhard Karls Universität Tübingen, Faculty of Science, Institute for Astronomy and Astrophysics. ${ }^{66}$ B) Cross-section of a CEM. Adapted from Penlink. ${ }^{67}$

\subsubsection{Hybrid Quadrupole Time-of-Flight Mass Spectrometer}

The instrument used in this thesis is the hybrid quadrupole time-of-flight mass spectrometer (QTOF-MS). A QTOF-MS is a highly sensitive, mass accurate MS that 
offers high resolution of ions with a wide dynamic range. Specifically, the QTOF-MS used throughout this thesis is the 6546 LC-QTOF from Agilent Technologies. A schematic diagram of the instrument is illustrated in Figure 1.19. The 6546 QTOF is equipped with a dual Jet Stream Technology ESI source (AJS), a hexapole collision cell, an ion beam shaper, and a TOF with an MCP detector. ${ }^{68}$

Much like the ESI described in Chapter 1.2.1, Agilent Technology's proprietary dual Jet Stream Technology ESI (AJS), shown in Figure 1.20, functions in the same manner. The dual portion indicates there are two lines connected to the source. The first one is connected to the HPLC where the separated analytes are sprayed at atmospheric pressure. The second line connects to the reference mass bottles where a solution of known $\mathrm{m} / \mathrm{z}$ can be simultaneously sprayed to perform minor adjustments to mass calibration. ${ }^{68}$ The second line was not used in this study. Once a sample is ionized, gaseous ions pass through the orifice along the ion path into the octupole ion guide. The octupole guides $100 \%$ of the ions into the mass filtering Q1 quadrupole. ${ }^{68}$ Ions with a target mass or target mass range are allowed to transmit through into the collision cell whereas non-target mass(es) do not. In the hexapole collision cell, parent ions are subjected to collisions with inert $\mathrm{N}_{2}(\mathrm{~g})$ molecules to generate fragments called daughter ions. This is also known as collision-induced dissociation (CID). ${ }^{40}$ Afterwards, ions enter the ion beam shaper where rods converge, focusing daughter ions on the entrance of the TOF ion accelerator. lons are separated in the TOF as previously described in Chapter 1.2.3 and get detected with an MCP detector, previously described in Chapter 1.2.4. 


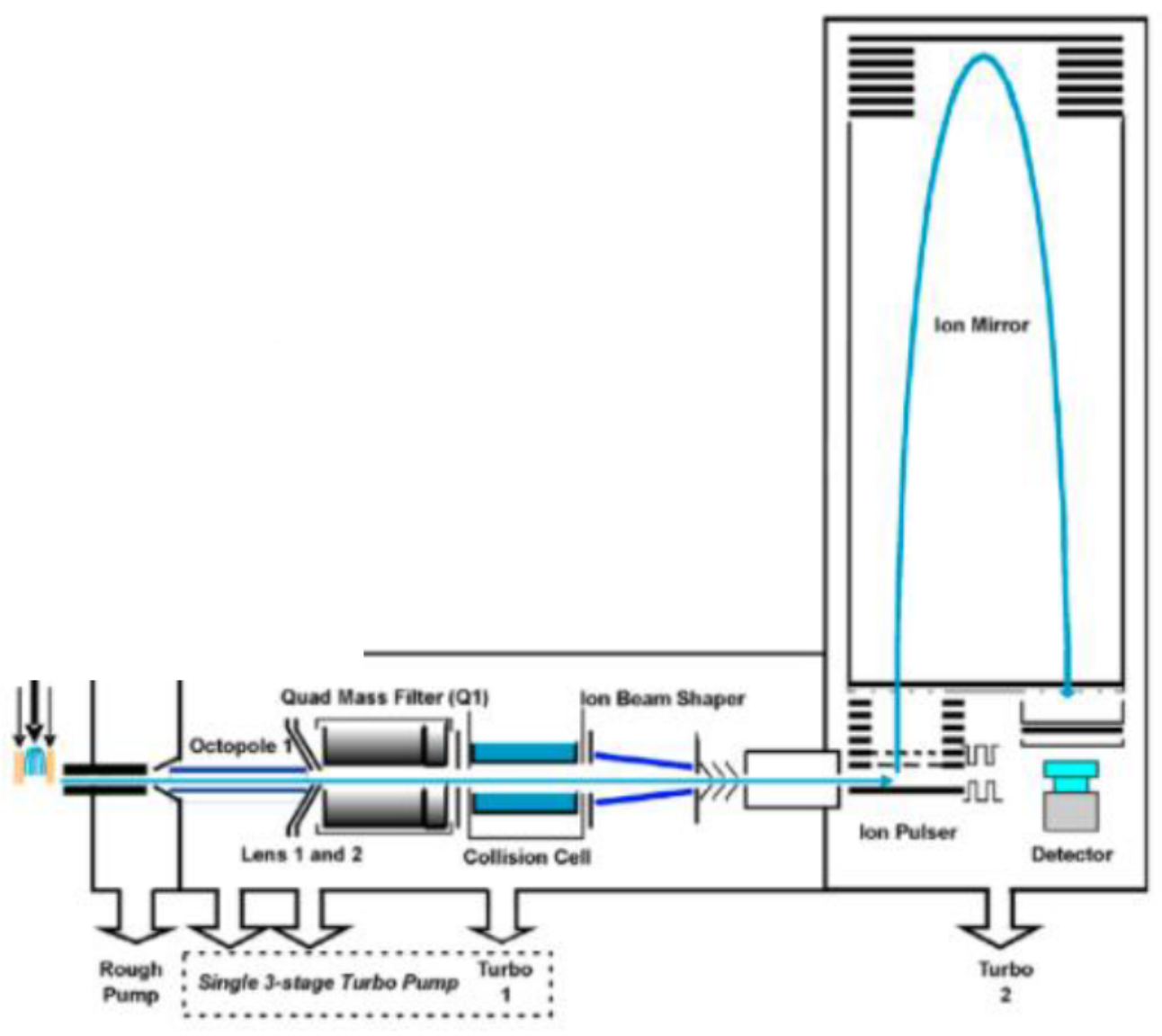

Figure 1.19: Schematic of Agilent's 6546 LC-QTOF. Figure adapted from Agilent Technologies (user manual). ${ }^{68}$

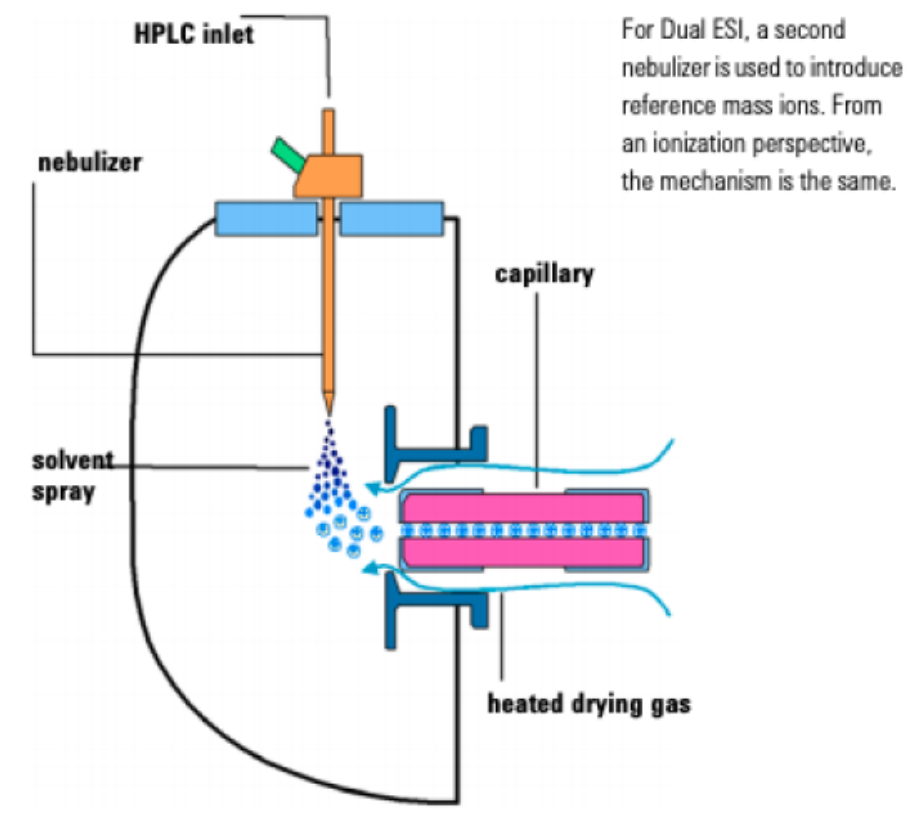

Figure 1.20: Schematic of Agilent's dual Jet Stream Technology electrospray ionization source. Figure adapted from Agilent Technologies (user manual). ${ }^{68}$ 


\subsubsection{MS-Based Lipidomics}

There are many commonly used techniques to analyze lipids. Direct infusion via ESI, simply called "shotgun" lipidomics and normal-phase or reversed-phase HPLC are two common methods to introduce lipids into a mass spectrometer. While direct fusion provides a simpler experimental setup and quicker analysis time, it is not ideal for global lipidome analysis. Certain lipid classes are more prevalent to ionize under a specific condition over others. This can consequentially lead to ion suppression ${ }^{69}$ of other lipid classes ${ }^{70}$ Salts, metabolites, proteins, and other contaminants can contribute to ion suppression ${ }^{69}$ thus limiting the amount of ionization that can occur at a particular moment through ESI. ${ }^{2}$ The work done in this thesis will utilize a reversed-phase HPLC to reduce ion suppression and contaminants.

Lipid structure elucidation is one of the many challenges in the field of lipidomics. ${ }^{2}$ Complete lipid structure characterization is highly dependent on interpretations of the mass spectrum produced; thus intact, native lipid species are required for proper identification. ${ }^{45}$ All "sections" of the lipid such as the polar head group and the fatty acyl tail must remain intact and must be in its natural, unmodified form for complete structure elucidation. ${ }^{2,71}$ This rarely is the case since lipids are constantly subjected to modifications like oxidation or decomposition, which presents a challenge in determining complete structural identification. ${ }^{2}$

Analysis of glycerophospholipids, fatty acyls, glycerolipids, and sphingolipids are typically performed using an ESI-MS coupled to an HPLC. ${ }^{2}$ For many glycerophospholipids and sphingolipids (PC, PE, PS, and SM), analysis is generally performed using positive ESI-MS/MS. ${ }^{72}$ The polarity, in which certain species are 
analyzed depends on the lipid species' gas-phase basicity. In positive polarity, the positive charge on the phosphorus readily forms an $[\mathrm{M}+\mathrm{H}]^{+}$ion from the electrospray process. ${ }^{2}$ In addition, lipid extracts from biological samples can be ionized as salt adducts, such as $[\mathrm{M}+\mathrm{Na}]^{+}$since the presence of sodium is common. Under CID, fragmentations from $\mathrm{PC}$, their lyso derivatives and SM yield a characteristic fragment of $\mathrm{m} / \mathrm{z} 184 .^{73}$ This fragment represents the phosphocholine head group and it highlights every phosphocholine-containing lipid (PC and SM). ${ }^{2,74}$ For PE species, they can readily form $[\mathrm{M}+\mathrm{H}]^{+}$or $[\mathrm{M}-\mathrm{H}]^{-}$ions depending on the polarity selected. In positive polarity, an $[\mathrm{M}+\mathrm{H}]^{+}$ ion yields a characteristic $[\mathrm{M}+\mathrm{H}-141]^{+}$fragment under $\mathrm{CID}$, which corresponds to the neutral loss of the phosphatidylethanolamine head group. ${ }^{2}$ Similarly, under positive ESI, protonated PS ion $[\mathrm{M}+\mathrm{H}]^{+}$also yield a neutral loss of the phosphoserine head group, resulting in a $[\mathrm{M}+\mathrm{H}-185]^{+}$fragment under $\mathrm{CID} .^{2,74}$

Structural elucidation of fatty acyls and neutral glycerolipids such as DGs and TGs can be performed using positive ESI-MS/MS. Both classes can form adducts with alkalimetal ions, mainly sodium since a large amount exists in biological extractions $[\mathrm{M}+\mathrm{Na}]^{+}$. Much like glycerophospholipids and sphingolipids, analysis of these classes with positive polarity will yield sodiated fragments. With unsaturated fatty acyls, CID may cause rearrangements to occur which is challenging to determine the complete structure. In addition to the $[\mathrm{M}+\mathrm{Na}]^{+}$ions, neutral glycerolipids can also form ammoniated $\left[\mathrm{M}+\mathrm{NH}_{4}\right]^{+}$ ions. ${ }^{75}$ However, the ionization efficiency of both TGs and DGs highly depends on the degree of unsaturation as well as the acyl chain length. ${ }^{2}$ Fragment ions of acyl chains from neutral glycerolipids also provide key information regarding the lipid structure. ${ }^{2}$ This helps in determining the lipid structure with high confidence. 
In MS-based lipidomics, it is critical to use an instrument that has high resolving power. This is because many lipid species possessing different combinations of acyl chain lengths and number of unsaturation can co-elute, making it difficult to distinguish or resolve under low resolving power MS. Additionally, the use of an HPLC is critical. Generally, reverse-phase HPLC is used in lipidomics. With normal phase, although classes of lipids can be separated with good resolution, isobaric species within a class will co-elute, decreasing the ability to identify different species. In reversed-phase, isobaric species can be separated since it is based on the hydrophobicity of the acyl chains. This results in isobaric species eluting at different times for MS-based identification.

In untargeted lipidomics, the goal is to determine as many lipid species in a sample as possible. One strategy is to employ iterative exclusion. This strategy overcomes traditional data dependent MS/MS in terms of the limited number of precursors selected for MS/MS at a given retention time. Iterative injection simply is the continuous repeat of MS/MS on the same sample but excludes the previously selected precursor ion in each subsequent analysis. This technique allows greater depth of information and better lipidomic coverage of a sample. ${ }^{76}$

\subsection{Separation via High Performance Liquid Chromatography}

Chromatographic techniques coupled with MS instruments create a highly robust combination for any analytic work. Previously in Chapter 1.1.3, liquid chromatography was described to be more favourable in lipidomics due to its versatility, high sensitivity, and better resolution. ${ }^{2}$ Separation of mixtures significantly improve overall results since the MS can focus on fewer ions at a time and highly abundant species are only limited to 
a certain small chromatographic window. This allows species of low abundance to be identified with less interference. ${ }^{2}$ With a complex biological sample in an untargeted scenario, this is especially useful. In addition, chromatographic techniques also separate any stereoisomers present in the sample. While there are many separation techniques available, some mentioned in Chapter 1.1.4, the reversed-phase HPLC will be the only one discussed since it was the only technique used in this thesis.

In RP-HPLC, lipids are separated based on their overall hydrophobicity. Generally, the shorter the acyl chain, the earlier the elution will be. ${ }^{2}$ RP-HPLC uses a non-polar stationary phase (the chromatographic column) and initially a polar mobile phase. As the analysis progresses, the mobile phase becomes increasingly non-polar. This results in more polar lipids, lipids with higher affinity to the mobile phase, eluting first and progressing to less polar lipids, lipids with higher affinity to the column. Further details regarding the solvent mixture for the mobile phase, the column used, and the parameters set will be presented in Chapter 2.2.

\subsection{Radiation}

Radiation, by definition, is the emission of energy in a form of either waves or particles. ${ }^{77}$ All forms of life have evolved from radiation-filled environments; we are constantly surrounded by many forms of radiation. ${ }^{78}$ The term radiation has often been misconstrued negatively through association with atomic energy, nuclear weaponry, and radioactive material when in fact, visible light, radio signals, and heat are also forms of radiation. ${ }^{78}$ Some applications of radiation are beneficial, especially in medical imaging and diagnosing procedures. However, these are strictly controlled to prevent any harm or damage. ${ }^{78,79}$ In Canada, government agencies such Canadian Nuclear Safety 
Commission (CNSC) and the Radiation Protection Bureau at Health Canada are responsible for mandating and delivering both environmental and occupational radiation protection programs. ${ }^{78}$ These programs exist to prevent any deleterious health effects due to radiation exposure. In the extreme, prolonged exposure or exposure to large amounts of radiation can cause serious side effects. Some include DNA damage, which leads to mutations and cancer $^{80}$, protein modification and degradation ${ }^{81}$, and lipid peroxidation ${ }^{82}$. In this thesis, the focus will be on the lattermost.

\subsubsection{Various Types of Radiation (ionizing/non-ionizing)}

Radiation generally is classified as either ionizing or non-ionizing based on the energy of the emitted particles. ${ }^{78}$ Figure 1.21 presents an overview of many forms of radiation, both ionizing and non-ionizing, in the electromagnetic spectrum. Non-ionizing radiation refers to any type of radiation that does not cause ionization. It is low energy and examples include visible light, radio waves, and microwaves. By contrast, ionizing radiation $(\mathrm{IR})$ is the high-energy emission of particles or waves that is capable of knocking electrons out of molecules or ionizing atoms. ${ }^{77}$ Examples of ionizing radiation include Xrays and gamma $(\gamma)$ rays which both participate in interactions with atoms causing particle ejection. ${ }^{77}$ These particle ejections can be classified into several types. Alpha radiation $(\alpha)$ emits alpha particles that comprise a helium nucleus (two protons and two neutrons). This particle carries two positive charges. Since alpha particles are large, they do not have the capacity to penetrate surfaces compared to other forms of radiation. A sheet of paper can stop an $\alpha$ particle, similarly, our skin can as well. Therefore, $\alpha$-emitting substances do not pose a radiation hazard if they are external to the body. However, if $\alpha$ emitting substances were to enter our body through inhalation or ingestion, the energy 
emitted is capable of producing health hazards since bodily tissues can absorb this energy readily. ${ }^{77}$

Another form of radiation is beta $(\beta)$ radiation which emits charged particles from an atom's nucleus. These charged particles can either be an electron or a positron (a positively charged particle with the same mass as an electron). ${ }^{77,78}$ Compared to alpha particles, beta particles can penetrate deeper, through outer layers of human skin but not into bodily tissues or organs; it can be stopped via shielding such as sheets of plastic or metal. ${ }^{77}$ Gamma $(\gamma)$ radiation and X-rays are high-energy photons that can penetrate deeply into matter. Dense materials such as lead, or steel only reduce the intensity and compared to beta particles, photon emission can penetrate deeply into bodily tissues and organs even if the source is outside the body. ${ }^{77}$ Finally, there is neutron $(n)$ radiation which occurs through spontaneous fission or from cosmic radiation. ${ }^{78} \mathrm{~A}$ summary of these four types of radiation and their penetration abilities are illustrated in Figure 1.22. An $\alpha$-emitting radiation source is used for the work done in this thesis.

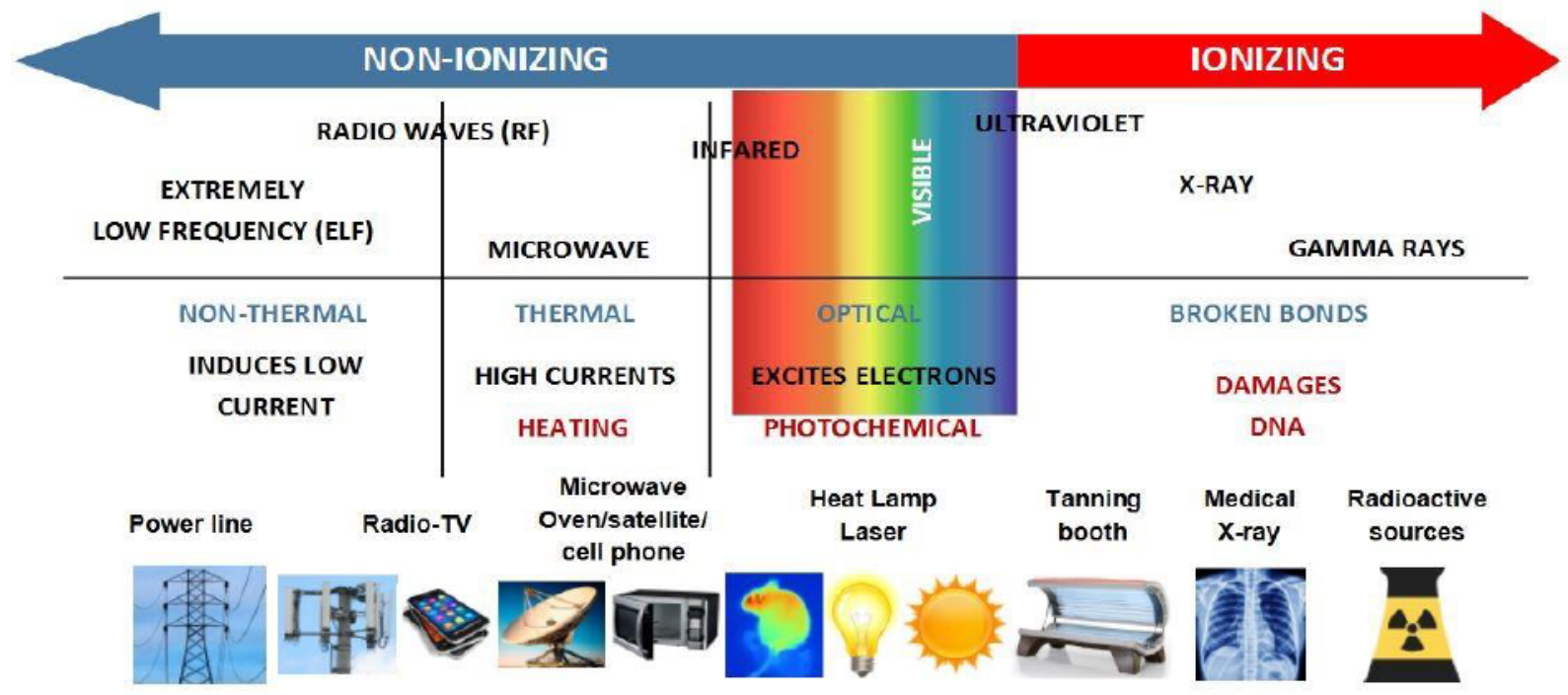

Figure 1.21: Electromagnetic Spectrum. Figure adapted from University of Washington, Environmental Health \& Safety. ${ }^{83}$ 


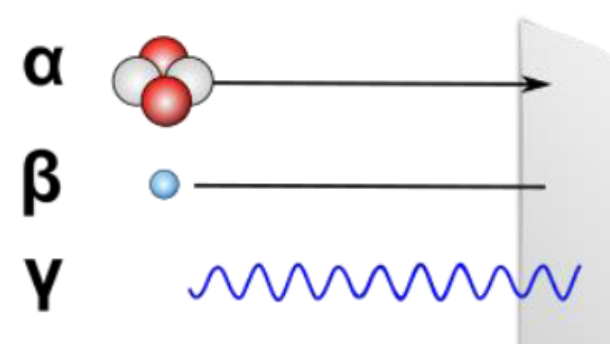

\section{neutron}

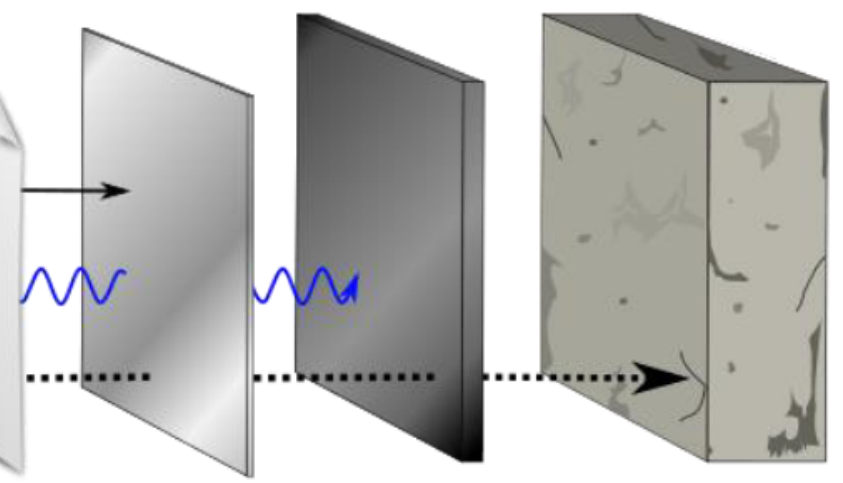

\section{PAPER ALUMINIUM LEAD CONCRETE}

Figure 1.22: Penetrating power of different types of radiation. Figure adapted from Jan Helebrant. ${ }^{84}$

The source of radiation used in this thesis work comes from Polonium-210 $\left({ }^{210} \mathrm{Po}\right)$. Polonium-210 $\left({ }^{210} \mathrm{Po}\right)$ is a radioactive element that occurs naturally through the decay of

natural uranium $\left({ }^{238} \mathrm{U}\right)$, and it is found at very low concentrations in the Earth's crust. ${ }^{85}$ ${ }^{210} \mathrm{Po}$ emits alpha particles and has a half-life of roughly 140 days, decaying into ${ }^{206} \mathrm{~Pb} .{ }^{86}$ Although ${ }^{210} \mathrm{Po}$ does not pose health hazards during uranium decay and outside the body, like any $\alpha$-emitting material, it presents a radiation hazard once inside the body through inhalation or consumption. ${ }^{85}$ Although humans do not directly consume ${ }^{210} \mathrm{Po}$, they exist in our ecosystem, contaminating our water and food supplies (ie. fish). ${ }^{85,87}$

Quantitation of radiation is typically measured in Becquerel $(\mathrm{Bq})$ and Sievert (Sv). Becquerel $(\mathrm{Bq})$ is the $\mathrm{SI}$ unit used to express the amount of radioactivity a particular substance gives off. $1 \mathrm{~Bq}$ is equivalent to one nucleus decay per second. ${ }^{78}$ This measurement focuses on the decay of a given material, correlating a larger value to a greater quantity of radiation released. Sv, on the other hand, describes the amount of radiation dose that a human receives. However, the radiation dosage depends on several factors. Factors include whether the exposure is internal or external of the body, whether 
the exposure is localized to a particular organ or the whole body, and the type of radiation. ${ }^{78}$ What is commonly expressed in literature is the effective dose (units: Sv). Figure 1.24 summarizes the relationship between the units. When radiation passes through a substance, the energy that is absorbed is appropriately called the absorbed dose which is recorded as Gray (Gy). Depending on the type of radiation, its effects vary. The equivalent dose accounts for the health effects of a particular type of radiation emission from a radioactive material. Finally, different tissues in the body will react differently when exposed to radiation. The effective dose (Sv) expresses the radiation doses in terms of the effects it has on a particular tissue or the whole body. ${ }^{78}$

In this thesis, the molecules of interest are lipids that are found in the liver. The liver is an organ of interest since it plays a key role in lipid metabolism. Much of the storage of TGs and fatty acid synthesis occurs in the liver, which makes a great candidate for this study. ${ }^{20}$ Additionally, the liver is a nearly homogenous organ; the composition and metabolic processes remain conserved throughout the entire organ. ${ }^{88}$

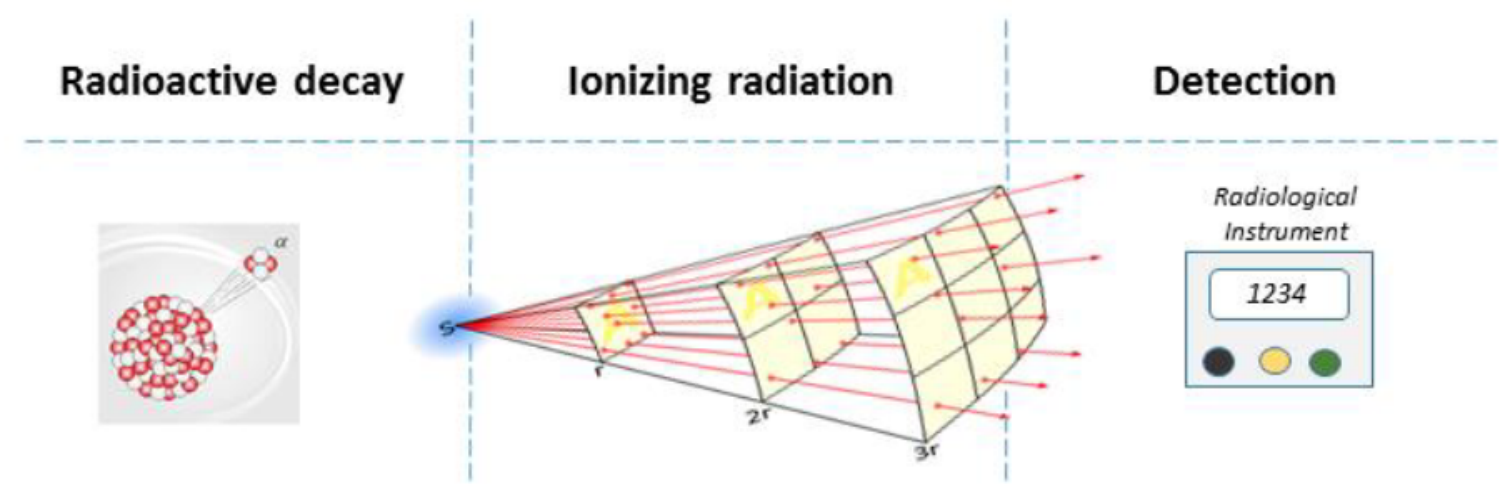

Figure 1.23: Relationship between radioactive decay and detection. Figure obtained from Wikipedia. ${ }^{89}$ 


\section{Units of Radiation Relationship between Units}

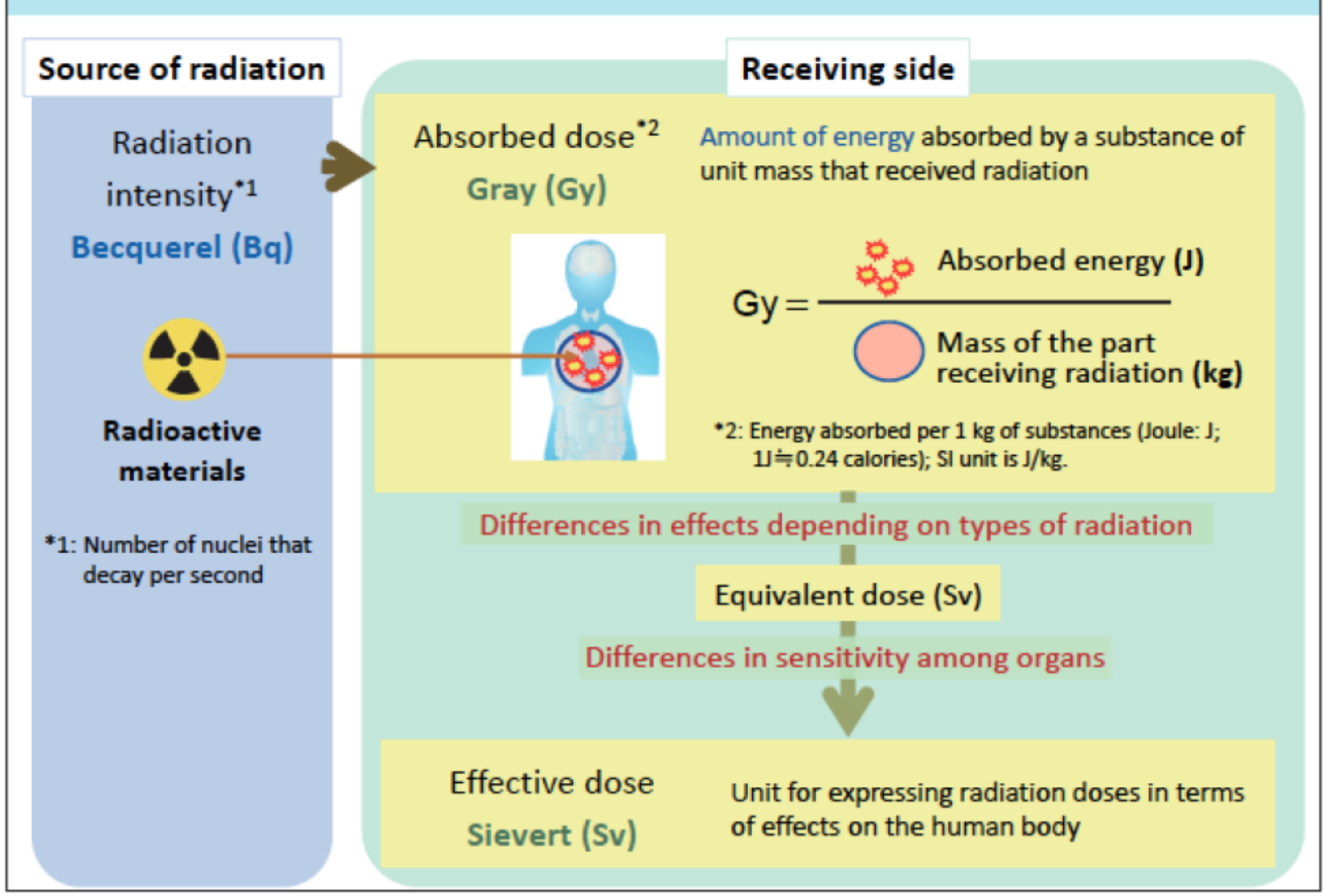

Figure 1.24: Relationship between radiation decay and dosage. Figure obtained from Ministry of the Environment, Government of Japan. ${ }^{90}$

\subsubsection{Radiation and Lipid Biochemistry}

Although there is substantial evidence that suggests that exposure to high dosages of radiation is harmful, the biological effects of low dose radiation (LDR) exposure are less well understood. LDR exposure is what is classified as "safe" although it does not mean complete absence of exposure, it is the level at which it does not pose harmful effects on a biological system. ${ }^{91}$ Although there is no definition of LDR, many experimental studies consider LDR as any ionizing radiation under 0.1-0.5 Gy.$^{79} \mathrm{LDR}$ is particularly useful in medical diagnoses through X-ray and computed tomography (CT) scans. The details of how these medical techniques work will not be discussed in this 
thesis. LDR exposure was once considered relatively harmless however, there is growing evidence that suggests that this might not be true. A study by Little et al. suggests that although LDR does not kill cells directly, mutations may still occur and initiate cancer. ${ }^{80}$ In addition to LDR exposure from medical devices, nuclear workers and uranium miners are also subjected to LDR. Prolong exposure increases risks due to cumulative effects. ${ }^{78,91}$

There have been many studies that determine the effects of LDR exposure on proteins and metabolites, however, there have been only a few studies that explore the effects of LDR on lipids. Although it is unclear whether LDR enhances or suppresses lipid oxidation, there have been studies that suggest continuous exposure to LDR, and accumulative effects contribute to a significant decrease in gene expression associated with non-esterified fatty acid metabolism ${ }^{79}$ as well as an overall imbalance in homeostasis with regards to lipid metabolism in the liver as a whole. ${ }^{79,92}$ Previous studies that explored the effects of ionizing radiation using ${ }^{60} \mathrm{Co}$, a $\gamma$ emitter ${ }^{82,93}$, and ${ }^{137} \mathrm{Cs}$, a $\beta$ \& $\gamma$ emitter ${ }^{81,94}$ resulted in an increase in TGs after irradiation in bone marrow cells of rabbits ${ }^{95}$, and specific TGs in serum of nonhuman primates ${ }^{96}$ which can be attributed to increased biosynthesis. Additionally, internal irradiation may result in the formation of reactive oxygen species (ROS) that contribute to lipid peroxidation and inflammation response via the lipoxygenase (LOX) and cyclooxygenase (COX) pathways ${ }^{96}$ Many of these studies attribute their findings to high energy internal radiation exposure but none of the studies focus on LDR. Although ${ }^{210} \mathrm{Po}$ poses little threat to human health in general, it is unclear whether $\alpha$ particle emitters at low-dose exposures pose any kind of health hazard, and specifically to the cellular lipidome. 


\subsection{Summary}

Within the field of lipidomics, there are many challenges associated with the separation, characterization and quantitation of many lipid classes, and species present in a cell. With potentially over 1000 lipid species in a cellular lipidome and 100,000 possible species, challenges arise from low-resolution instruments, unquantifiable methods, and lack of structural elucidation of lipids species. LC-MS/MS analyses provide a robust analytical technique to conduct many lipidomic studies as it is highly sensitive and has greater resolving power than previous methodologies. This is helpful with studying peroxide lipids. It is known that lipid peroxidation occurs from radiation exposure however, very little is known about the effect of LDR exposure on the lipidome. LDR exposure once thought to be relatively harmless, may attribute to inflammatory response and homeostasis imbalance with lipid metabolism. In light of this knowledge gap, this thesis explores the effects of LDR via a $\alpha$ particle emitter on a Wistar rat liver lipidome. To accomplish this, two objectives were pursued and accomplished: first, validating the methodology, and secondly, performing a comprehensive MS-based quantitative lipidomics analysis. The following chapters describe the methodologies, experiments, and results of LDR exposure on a lipidome. Chapter 2 describes the extraction and instrumental methods used throughout this study. Chapter 3 explores the optimization approach to the lipidomics workflow as well as providing some insight into MS analysis with both bovine liver lipid extracts and Wistar rat liver lipid extract. Chapter 4 describes the effects of LDR exposure on a liver lipidome. 


\subsection{References}

(1) Voet, D.; Voet, J. G. Biochemistry, 4th ed.; John Wiley \& Sons: Hoboken, NJ, 2011.

(2) Bou Khalil, M.; Hou, W.; Zhou, H.; Elisma, F.; Swayne, L. A.; Blanchard, A. P.; Yao, Z.; Bennett, S. A. L.; Figeys, D. Lipidomics Era: Accomplishments and Challenges: LIPIDOMICS ERA: ACCOMPLISHMENTS AND CHALLENGES. Mass Spectrom. Rev. 2010, 29 (6), 877-929. https://doi.org/10.1002/mas.20294.

(3) Fahy, E.; Subramaniam, S.; Murphy, R. C.; Nishijima, M.; Raetz, C. R. H.; Shimizu, T.; Spener, F.; van Meer, G.; Wakelam, M. J. O.; Dennis, E. A. Update of the LIPID MAPS Comprehensive Classification System for Lipids. J. Lipid Res. 2009, April Supplementary, S9-S14. https://doi.org/10.1194/jlr.R800095-JLR200.

(4) Subramaniam, S.; Fahy, E.; Gupta, S.; Sud, M.; Byrnes, R. W.; Cotter, D.; Dinasarapu, A. R.; Maurya, M. R. Bioinformatics and Systems Biology of the Lipidome. Chem. Rev. 2011, 111 (10), 6452-6490.

https://doi.org/10.1021/cr200295k.

(5) Wenk, M. R. The Emerging Field of Lipidomics. Nat. Rev. Drug Discov. 2005, 4 (7), 594-610. https://doi.org/10.1038/nrd1776.

(6) Blank, K. Targeted Quantitative Lipidomics of Cold Stress and the Development of Methods to Increase the Sensitivity of Proteomics Analyses Using Mass Spectrometry, Carleton University, Ottawa, 2018.

(7) van Eyk, H. J.; van Schinkel, L. D.; Kantae, V.; Dronkers, C. E. A.; Westenberg, J. J. M.; de Roos, A.; Lamb, H. J.; Jukema, J. W.; Harms, A. C.; Hankemeier, T.; van der Stelt, M.; Jazet, I. M.; Rensen, P. C. N.; Smit, J. W. A. Caloric Restriction Lowers Endocannabinoid Tonus and Improves Cardiac Function in Type 2 Diabetes. Nutr. Diabetes 2018, 8 (1), 6. https://doi.org/10.1038/s41387-017-00167.

(8) Grundy, S.; Denke, M. Dietary Influences on Serum Lipids and Lipoproteins. J. Lipid Res. 1990, 31 (7), 1149-1172. https://doi.org/10.1016/S0022-2275(20)426252.

(9) Proitsi, P.; Kim, M.; Whiley, L.; Simmons, A.; Sattlecker, M.; Velayudhan, L.; Lupton, M. K.; Soininen, H.; Kloszewska, I.; Mecocci, P.; Tsolaki, M.; Vellas, B.; Lovestone, S.; Powell, J. F.; Dobson, R. J. B.; Legido-Quigley, C. Association of Blood Lipids with Alzheimer's Disease: A Comprehensive Lipidomics Analysis. Alzheimers Dement. 2017, 13 (2), 140-151. https://doi.org/10.1016/j.jalz.2016.08.003.

(10) Mayberg, M. R.; Batjer, H. H.; Dacey, R.; Diringer, M.; Haley, E. C.; Heros, R. C.; Sternau, L. L.; Tomer, J.; Jr, H. P. A.; Feinberg, W.; Thies, W. Guidelines for the 
Management of Aneurysmal Subarachnoid Hemorrhage. A Statement for Healthcare Professionals from a Special Writing Group of the Stroke Council, American Heart Association. Stroke 1994, 25 (14), 2315-2328.

(11) Pawar, A. B.; Sengupta, D. Effect of Membrane Composition on Receptor Association: Implications of Cancer Lipidomics on ErbB Receptors. J. Membr. Biol. 2018, 251 (3), 359-368. https://doi.org/10.1007/s00232-018-0015-1.

(12) Panahi, Y.; Ahmadi, Y.; Teymouri, M.; Johnston, T. P.; Sahebkar, A. Curcumin as a Potential Candidate for Treating Hyperlipidemia: A Review of Cellular and Metabolic Mechanisms. J. Cell. Physiol. 2018, 233 (1), 141-152. https://doi.org/10.1002/jcp.25756.

(13) McLaughlin, K. Cell Membrane. Biodictionary; 2017.

(14) van Meer, G. Cellular Lipidomics. EMBO J. 2005, 24 (18), 3159-3165. https://doi.org/10.1038/sj.emboj.7600798.

(15) Shields, S. W. J.; Canez, C. R.; Wasslen, K. V.; Lee, H.; Stalinski, D.; Trouborst, L.; Joudan, S.; Whitton, S.; Weinert, H. P.; Manthorpe, J. M.; Smith, J. C. Enhancing the Analysis of Complex Lipid Samples Through Developments in Chromatography and Chemical Derivatization. In Molecular Technologies for Detection of Chemical and Biological Agents; Banoub, J. H., Caprioli, R. M., Eds.; NATO Science for Peace and Security Series A: Chemistry and Biology; Springer Netherlands: Dordrecht, 2017; pp 177-206. https://doi.org/10.1007/978-94-024-1113-3_11.

(16) Fahy, E.; Subramaniam, S.; Brown, H. A.; Glass, C. K.; Merrill, A. H.; Murphy, R. C.; Raetz, C. R. H.; Russell, D. W.; Seyama, Y.; Shaw, W.; Shimizu, T.; Spener, F.; van Meer, G.; VanNieuwenhze, M. S.; White, S. H.; Witztum, J. L.; Dennis, E. A. A Comprehensive Classification System for Lipids. J. Lipid Res. 2005, 46 (5), 839861. https://doi.org/10.1194/jlr.E400004-JLR200.

(17) Wang, H.-M. D.; Li, X.-C.; Lee, D.-J.; Chang, J.-S. Potential Biomedical Applications of Marine Algae. Bioresour. Technol. 2017, 244, 1407-1415. https://doi.org/10.1016/j.biortech.2017.05.198.

(18) Hunter, J. E. Dietary Trans Fatty Acids: Review of Recent Human Studies and Food Industry Responses. Lipids 2006, 41 (11), 967-992. https://doi.org/10.1007/s11745-006-5049-y.

(19) Prentki, M.; Madiraju, S. R. M. Glycerolipid Metabolism and Signaling in Health and Disease. Endocr. Rev. 2008, 29 (6), 647-676. https://doi.org/10.1210/er.20080007.

(20) Alves-Bezerra, M.; Cohen, D. E. Triglyceride Metabolism in the Liver. Compr. Physiol. 2019, 8 (1), 1-8. https://doi.org/10.1002/cphy.e170012. 
(21) Subczynski, W. K.; Pasenkiewicz-Gierula, M.; Widomska, J.; Mainali, L.; Raguz, M. High Cholesterol/Low Cholesterol: Effects in Biological Membranes: A Review. Cell Biochem. Biophys. 2017, 75, 369-385. https://doi.org/10.1007/s12013-017-0792-7.

(22) Tsai, M.-J.; O'Malley, B. W. MOLECULAR MECHANISMS OF ACTION OF STEROID/THYROID RECEPTOR SUPERFAMILY MEMBERS. Annu. Rev. Inc 1994, 63, 39.

(23) Foti, M. C. Antioxidant Properties of Phenols. J. Pharm. Pharmacol. 2010, 59 (12), 1673-1685. https://doi.org/10.1211/jpp.59.12.0010.

(24) Meganathan, R. Biosynthesis of Menaquinone (Vitamin K2) and Ubiquinone (Coenzyme Q): A Perspective on Enzymatic Mechanisms. In Vitamins \& Hormones; Elsevier, 2001; Vol. 61, pp 173-218. https://doi.org/10.1016/S00836729(01)61006-9.

(25) Rao, A.; Rao, L. Carotenoids and Human Health. Pharmacol. Res. 2007, 55 (3), 207-216. https://doi.org/10.1016/j.phrs.2007.01.012.

(26) Raetz, C. R. H.; Whitfield, C. Lipopolysaccharide Endotoxins. Annu. Rev. Biochem. 2002, 71 (1), 635-700.

https://doi.org/10.1146/annurev.biochem.71.110601.135414.

(27) Carlos Ramon Canez-Quijada. Increasing the Sensitivity of Phospholipid Analyses from Biological Extracts via Trimethylation Enhancement Using Diazomethane (TrEnDi) and Tandem Mass Spectrometry, Carleton University, Ottawa, 2015.

(28) Walsh, C. T. Polyketide and Nonribosomal Peptide Antibiotics: Modularity and Versatility. Science 2004, 303 (5665), 1805-1810. https://doi.org/10.1126/science.1094318.

(29) Hernandez, E. M.; Kamal-Eldin, A. Processing and Nutrition of Fats and Oils, 1st ed.; Wiley, 2013. https://doi.org/10.1002/9781118528761.

(30) Piomelli, D.; Astarita, G.; Rapaka, R. A Neuroscientist's Guide to Lipidomics. Nat. Rev. Neurosci. 2007, 8 (10), 743-754. https://doi.org/10.1038/nrn2233.

(31) Merrill, A. H.; Sandhoff, K. Chapter 14 Sphingolipids: Metabolism and Cell Signaling. In New Comprehensive Biochemistry; Elsevier, 2002; Vol. 36, pp 373407. https://doi.org/10.1016/S0167-7306(02)36016-2.

(32) Liebisch, G.; Vizcaíno, J. A.; Köfeler, H.; Trötzmüller, M.; Griffiths, W. J.; Schmitz, G.; Spener, F.; Wakelam, M. J. O. Shorthand Notation for Lipid Structures Derived from Mass Spectrometry. J. Lipid Res. 2013, 54, 1523-1530.

https://doi.org/10.1194/jr.M033506. 
(33) Rustam, Y. H.; Reid, G. E. Analytical Challenges and Recent Advances in Mass Spectrometry Based Lipidomics. Anal. Chem. 2018, 90 (1), 374-397. https://doi.org/10.1021/acs.analchem.7b04836.

(34) Bligh, E. G.; Dyer, W. J. A RAPID METHOD OF TOTAL LIPID EXTRACTION AND PURIFICATION. Can J Biochem Physiol 1959, 37 (8), 911-917.

(35) Folch, J.; Lees, M.; Stanley, G. H. S. A SIMPLE METHOD FOR THE ISOLATION AND PURIFICATION OF TOTAL LIPIDES FROM ANIMAL TISSUES. J. Biol. Chem. 1957, 226 (1), 497-509. https://doi.org/10.1016/S0021-9258(18)64849-5.

(36) Matyash, V.; Liebisch, G.; Kurzchalia, T. V.; Shevchenko, A.; Schwudke, D. Lipid Extraction by Methyl-Tert-Butyl Ether for High-Throughput Lipidomics. J. Lipid Res. 2008, 49 (5), 1137-1146. https://doi.org/10.1194/jr.D700041-JLR200.

(37) Ulmer, C. Z.; Jones, C. M.; Yost, R. A.; Garrett, T. J.; Bowden, J. A. Optimization of Folch, Bligh-Dyer, and Matyash Sample-to-Extraction Solvent Ratios for Human Plasma-Based Lipidomics Studies. Anal. Chim. Acta 2018, 1037, 351-357. https://doi.org/10.1016/j.aca.2018.08.004.

(38) Ramstedt, B.; Leppimäki, P.; Axberg, M.; Slotte, J. P. Analysis of Natural and Synthetic Sphingomyelins Using High-Performance Thin-Layer Chromatography: Analysis of Sphingomyelins by HPTLC. Eur. J. Biochem. 1999, 266 (3), 997-1002. https://doi.org/10.1046/j.1432-1327.1999.00938.x.

(39) Fuchs, B.; Süß, R.; Teuber, K.; Eibisch, M.; Schiller, J. Lipid Analysis by Thin-Layer Chromatography-A Review of the Current State. J. Chromatogr. A 2011, 1218 (19), 2754-2774. https://doi.org/10.1016/j.chroma.2010.11.066.

(40) Harris, D. C.; Lucy, C. A. Quantitative Chemical Analysis, Ninth edition.; W.H. Freeman \& Company: New York, 2016.

(41) Byreddy, A. R.; Gupta, A.; Barrow, C. J.; Puri, M. A Quick Colorimetric Method for Total Lipid Quantification in Microalgae. J. Microbiol. Methods 2016, 125, 28-32. https://doi.org/10.1016/j.mimet.2016.04.002.

(42) Cheng, Y.-S.; Zheng, Y.; VanderGheynst, J. S. Rapid Quantitative Analysis of Lipids Using a Colorimetric Method in a Microplate Format. Lipids 2011, 46 (1), 95103. https://doi.org/10.1007/s11745-010-3494-0.

(43) Abdullah, S.; Davies, S.; Wall, R. Spectrophotometric Analysis of Lipid Used to Examine the Phenology of the Tick Ixodes Ricinus. Parasit. Vectors 2018, 11 (1), 523. https://doi.org/10.1186/s13071-018-3102-3. 
(44) Greenspan, P.; Mayer, E. P.; Fowler, S. D. Nile Red: A Selective Fluorescent Stain for Intracellular Lipid Droplets. J. Cell Biol. 1985, 100 (3), 965-973.

https://doi.org/10.1083/jcb.100.3.965.

(45) Wu, Z.; Bagarolo, G. I.; Thoröe-Boveleth, S.; Jankowski, J. "Lipidomics": Mass Spectrometric and Chemometric Analyses of Lipids. Adv. Drug Deliv. Rev. 2020, 159, 294-307. https://doi.org/10.1016/j.addr.2020.06.009.

(46) Banerjee, S. Empowering Clinical Diagnostics with Mass Spectrometry. ACS Omega 2020, 5 (5), 2041-2048. https://doi.org/10.1021/acsomega.9b03764.

(47) Black, C.; D'Souza, T.; Smith, J. C.; Hearns, N. G. R. Identification of Post-Blast Explosive Residues Using Direct-Analysis-in-Real-Time and Mass Spectrometry (DART-MS). Forensic Chem. 2019, 16, 100185. https://doi.org/10.1016/j.forc.2019.100185.

(48) Gross, J. H. Mass Spectrometry; Springer Berlin Heidelberg: Berlin, Heidelberg, 2011. https://doi.org/10.1007/978-3-642-10711-5.

(49) Sharma, K. S. Mass Spectrometry-The Early Years. Int. J. Mass Spectrom. 2013, 349-350, 3-8. https://doi.org/10.1016/j.ijms.2013.05.028.

(50) Banerjee, S.; Mazumdar, S. Electrospray lonization Mass Spectrometry: A Technique to Access the Information beyond the Molecular Weight of the Analyte. Int. J. Anal. Chem. 2012, 2012, 1-40. https://doi.org/10.1155/2012/282574.

(51) Wang, Y.; Sun, J.; Qiao, J.; Ouyang, J.; Na, N. A "Soft" and "Hard" lonization Method for Comprehensive Studies of Molecules. Anal. Chem. 2018, 90 (24), 14095-14099. https://doi.org/10.1021/acs.analchem.8b04437.

(52) Steckel, A.; Schlosser, G. An Organic Chemist's Guide to Electrospray Mass Spectrometric Structure Elucidation. Molecules 2019, 24 (3), 611. https://doi.org/10.3390/molecules24030611.

(53) Glish, G. L.; Vachet, R. W. The Basics of Mass Spectrometry in the Twenty-First Century. Nat. Rev. Drug Discov. 2003, 2 (2), 140-150. https://doi.org/10.1038/nrd1011.

(54) Awad, H.; Khamis, M. M.; El-Aneed, A. Mass Spectrometry, Review of the Basics: Ionization. Appl. Spectrosc. Rev. 2015, 50 (2), 158-175. https://doi.org/10.1080/05704928.2014.954046.

(55) Miller, P. E.; Denton, M. B. The Quadrupole Mass Filter: Basic Operating Concepts. J. Chem. Educ. 1986, 63 (7), 617. https://doi.org/10.1021/ed063p617. 
(56) Smith, J. C. MASS SPECTROMETRY-BASED PROTEOMICS: NON-COVALENT INTERACTIONS AND PROTEIN IDENTIFICATION, York University, Toronto, 2005.

(57) Tang, X.; Bruce, J. E.; Hill, H. H. Characterizing Electrospray lonization Using Atmospheric Pressure lon Mobility Spectrometry. Anal. Chem. 2006, 78 (22), 7751-7760. https://doi.org/10.1021/ac0613380.

(58) Christian, G. D.; Dasgupta, P. K.; Schug, K. Analytical Chemistry, Seventh edition.; John Wiley and Sons, Inc: Hoboken, NJ, 2014.

(59) Douglas, D. J. Linear Quadrupoles in Mass Spectrometry. Mass Spectrom. Rev. 2009, 28 (6), 937-960. https://doi.org/10.1002/mas.20249.

(60) Shimadzu Scientific Korea Corp. Schematic of How Quadrupole MS Works.

(61) Wasslen, K. Trimethylation Enhancement Using Diazomethane (TrEnDi): A Novel Technique to Enhance the Sensitivity of MS-Based Analyses of Biological Molecules. Master of Science, Carleton University, Ottawa, Ontario, 2014. https://doi.org/10.22215/etd/2014-11212.

(62) Guilhaus, M.; Selby, D.; Mlynski, V. Orthogonal Acceleration Time-of-flight Mass Spectrometry. Mass Spectrom. Rev. 2000, 19, 65-107.

(63) Dodonov, A. F.; Kozlovski, V. I.; Soulimenkov, I. V.; Raznikov, V. V.; Loboda, A. V.; Zhen, Z.; Horwath, T.; Wollnik, H. High-Resolution Electrospray lonization Orthogonal-Injection Time-of-Flight Mass Spectrometer. Eur. J. Mass Spectrom. 2000, 6 (6), 481-490. https://doi.org/10.1255/ejms.378.

(64) Smith, J. C. High Resolution Mass Separation and Ion Detection, 2020.

(65) Wiza, J. L. MICROCHANNEL PLATE DETECTORS. Nucl. Instrum. Methods 1979, $162,587-601$.

(66) Eberhard Karls Universität Tübingen, Faculty of Science, Institute for Astronomy and Astrophysics. Development of UV MCP Detectors.

(67) Penlink. Channel Electron Multiplier (CEM); 2020.

(68) Agilent Technologies, Inc. Agilent 6200 Series TOF and 6500 Series Q-TOF LCMS System. 2017.

(69) Annesley, T. M. Ion Suppression in Mass Spectrometry. Clin. Chem. 2003, 49 (7), 1041-1044. https://doi.org/10.1373/49.7.1041. 
(70) Forest, A.; Ruiz, M.; Bouchard, B.; Boucher, G.; Gingras, O.; Daneault, C.; Robillard Frayne, I.; Rhainds, D.; The iGenoMed Consortium; The NIDDK IBD Genetics Consortium; Tardif, J.-C.; Rioux, J. D.; Des Rosiers, C. Comprehensive and Reproducible Untargeted Lipidomic Workflow Using LC-QTOF Validated for Human Plasma Analysis. J. Proteome Res. 2018, 17 (11), 3657-3670. https://doi.org/10.1021/acs.jproteome.8b00270.

(71) Köfeler, H. C.; Fauland, A.; Rechberger, G. N.; Trötzmüller, M. Mass Spectrometry Based Lipidomics: An Overview of Technological Platforms. Metabolites 2012, 2 (1), 19-38. https://doi.org/10.3390/metabo2010019.

(72) Retra, K.; Bleijerveld, O. B.; van Gestel, R. A.; Tielens, A. G. M.; van Hellemond, J. J.; Brouwers, J. F. A Simple and Universal Method for the Separation and Identification of Phospholipid Molecular Species. Rapid Commun. Mass Spectrom. 2008, 22 (12), 1853-1862. https://doi.org/10.1002/rcm.3562.

(73) Hsu, F.-F. Mass Spectrometry-Based Shotgun Lipidomics - a Critical Review from the Technical Point of View. Anal. Bioanal. Chem. 2018, 410 (25), 6387-6409. https://doi.org/10.1007/s00216-018-1252-y.

(74) Pi, J.; Wu, X.; Feng, Y. Fragmentation Patterns of Five Types of Phospholipids by Ultra-High-Performance Liquid Chromatography Electrospray lonization Quadrupole Time-of-Flight Tandem Mass Spectrometry. Anal. Methods 2016, 8 (6), 1319-1332. https://doi.org/10.1039/C5AY00776C.

(75) Chen, J.; Green, K. B.; Nichols, K. K. Quantitative Profiling of Major Neutral Lipid Classes in Human Meibum by Direct Infusion Electrospray lonization Mass Spectrometry. Investig. Opthalmology Vis. Sci. 2013, 54 (8), 5730. https://doi.org/10.1167/iovs.12-10317.

(76) Koelmel, J. P.; Kroeger, N. M.; Gill, E. L.; Ulmer, C. Z.; Bowden, J. A.; Patterson, R. E.; Yost, R. A.; Garrett, T. J. Expanding Lipidome Coverage Using LC-MS/MS Data-Dependent Acquisition with Automated Exclusion List Generation. J. Am. Soc. Mass Spectrom. 2017, 28 (5), 908-917. https://doi.org/10.1007/s13361-0171608-0.

(77) Donya, M.; Radford, M.; ElGuindy, A.; Firmin, D.; Yacoub, M. H. Radiation in Medicine: Origins, Risks and Aspirations. Glob. Cardiol. Sci. Pract. 2014, 2014 (4), 57. https://doi.org/10.5339/gcsp.2014.57.

(78) Introduction to Radiation; Canadian Nuclear Safety Commission, Ed.; Canadian Nuclear Safety Commission: Ottawa, 2012.

(79) Shin, E.; Lee, S.; Kang, H.; Kim, J.; Kim, K.; Youn, H.; Jin, Y. W.; Seo, S.; Youn, B. Organ-Specific Effects of Low Dose Radiation Exposure: A Comprehensive Review. Front. Genet. 2020, 11, 1-11. https://doi.org/10.3389/fgene.2020.566244. 
(80) Little, M. P.; Wakeford, R.; Tawn, E. J.; Bouffler, S. D.; Berrington de Gonzalez, A. Risks Associated with Low Doses and Low Dose Rates of lonizing Radiation: Why Linearity May Be (Almost) the Best We Can Do. Radiology 2009, 251 (1), 6-12. https://doi.org/10.1148/radiol.2511081686.

(81) Goudarzi, M.; Weber, W. M.; Mak, T. D.; Chung, J.; Doyle-Eisele, M.; Melo, D. R.; Brenner, D. J.; Guilmette, R. A.; Fornace, A. J. Metabolomic and Lipidomic Analysis of Serum from Mice Exposed to an Internal Emitter, Cesium-137, Using a Shotgun LC-MS E Approach. J. Proteome Res. 2015, 14 (1), 374-384. https://doi.org/10.1021/pr500913n.

(82) Pannkuk, E. L.; Laiakis, E. C.; Authier, S.; Wong, K.; Fornace, A. J. Targeted Metabolomics of Nonhuman Primate Serum after Exposure to lonizing Radiation: Potential Tools for High-Throughput Biodosimetry. RSC Adv. 2016, 6, 5119251202. https://doi.org/10.1039/C6RA07757A.

(83) UW Environmental Health \& Safety Department. Electromagnetic Spectrum.

(84) Jan Helebrant. Penetrating Power of Different Types of Radiation.

(85) Seiler, R. L.; Wiemels, J. L. Occurrence of 210 Po and Biological Effects of LowLevel Exposure: The Need for Research. Environ. Health Perspect. 2012, 120 (9), 1230-1237. https://doi.org/10.1289/ehp.1104607.

(86) Zagà, V.; Lygidakis, C.; Chaouachi, K.; Gattavecchia, E. Polonium and Lung Cancer. J. Oncol. 2011, 2011, 1-11. https://doi.org/10.1155/2011/860103.

(87) Lanzola, E. E.; Allegrini, M. E.; Taylor, D. M. The Binding of Polonium-210 to Rat Tissues. Radiat. Res. 1973, 56 (2), 370-384. https://doi.org/10.2307/3573673.

(88) Martín-Garre, S. Liver and Cardiovascular Disease: What Cardiologists Need to Know About Ultrasound Findings. Rev. Esp. Cardiol. Engl. Ed. 2017, 70 (5), 399401. https://doi.org/10.1016/j.rec.2016.09.013.

(89) Doug Sim. Graphic Showing Relationships between Radioactivity and Detected lonizing Radiation; 2014.

(90) Ministry of the Environment Government of Japan. Relationship between Units; 2013.

(91) Maqsudur Rashid, A.; Ramalingam, L.; Al-Jawadi, A.; Moustaid-Moussa, N.; Moussa, H. Low Dose Radiation, Inflammation, Cancer and Chemoprevention. Int. J. Radiat. Biol. 2019, 95 (4), 506-515. https://doi.org/10.1080/09553002.2018.1484194. 
(92) Azab, K.; Bashandy, M.; Salem, M.; Ahmed, O.; Tawfik, Z.; Helal, H. Royal Jelly Modulates Oxidative Stress and Tissue Injury in Gamma Irradiated Male Wister Albino Rats. North Am. J. Med. Sci. 2011, 3 (6), 268-276. https://doi.org/10.4297/najms.2011.3268.

(93) Pannkuk, E. L.; Laiakis, E. C.; Mak, T. D.; Astarita, G.; Authier, S.; Wong, K.; Fornace, A. J. A Lipidomic and Metabolomic Serum Signature from Nonhuman Primates Exposed to lonizing Radiation. Metabolomics 2016, 12 (80), 11. https://doi.org/10.1007/s11306-016-1010-0.

(94) Goudarzi, M.; Weber, W.; Mak, T. D.; Chung, J.; Doyle-Eisele, M.; Melo, D.; Brenner, D. J.; Guilmette, R. A.; Fornace, A. J. Development of Urinary Biomarkers for Internal Exposure by Cesium-137 Using a Metabolomics Approach in Mice. Radiat. Res. 2013, 181 (1), 54. https://doi.org/10.1667/RR13479.1.

(95) Wooles, W. R. Triglyceride Synthesis Following Whole-Body X-Irradiation in the Rabbit. Radiat. Res. 1967, 30 (4), 788. https://doi.org/10.2307/3572144.

(96) Pannkuk, E. L.; Laiakis, E. C.; Singh, V. K.; Fornace, A. J. Lipidomic Signatures of Nonhuman Primates with Radiation-Induced Hematopoietic Syndrome. Sci. Rep. 2017, 7 (9777), 9. https://doi.org/10.1038/s41598-017-10299-w. 


\section{Chapter 2: Materials and Methodology}

The materials and methods in this chapter are generalized for the various experiments performed in this thesis. Detailed methodologies can be found in individual chapters.

\subsection{Lipids Studied in this Thesis}

For the purpose of this research, positive ion mode was used to identify and quantify glycerolipids (TG, DG), sphingolipids (SM, ceramides), fatty acids (CE) and, glycerophospholipids (PC, PE, LPC).

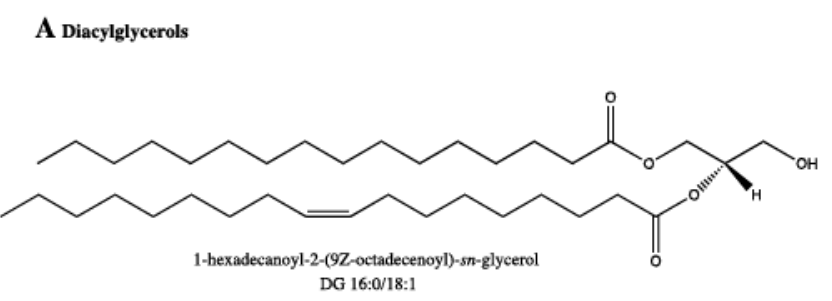

C Ceramides
B Triacylglycerols

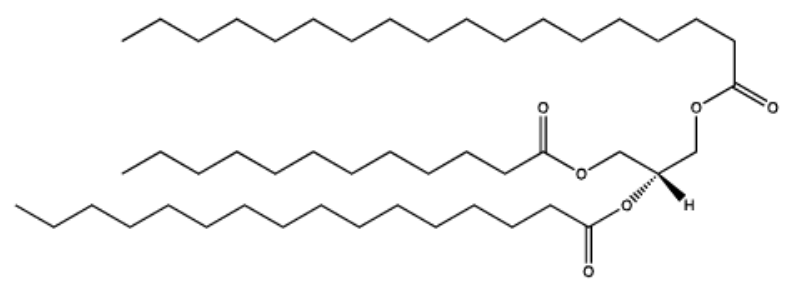

1-O-(1Z-tetraadecenyl)-2-(9Z-octadecenoyl)-sn-glycerol
TG 12:0/16:0/18:0

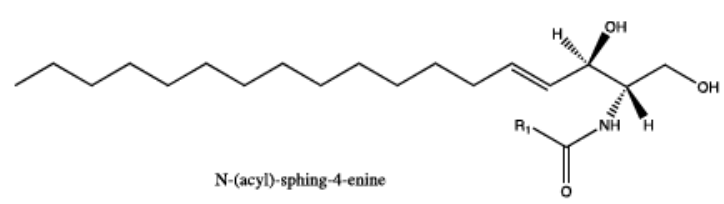

D Phosphosphingolipids

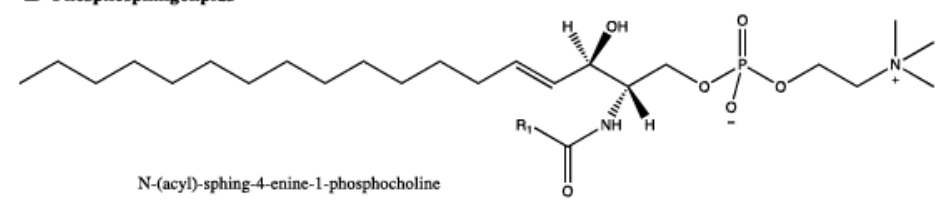

Continues on the next page 

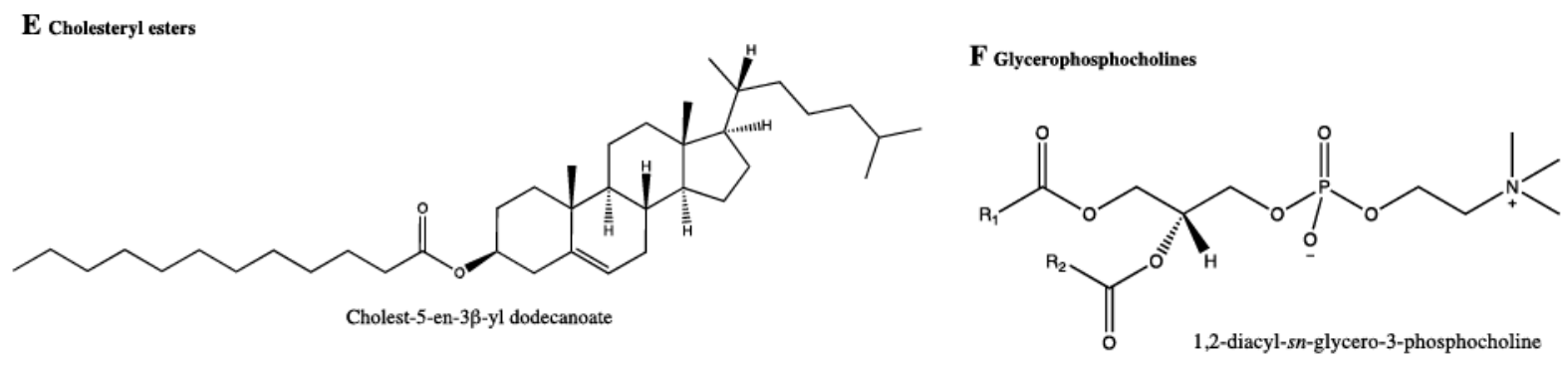

G Glycerophosphoethanolamines
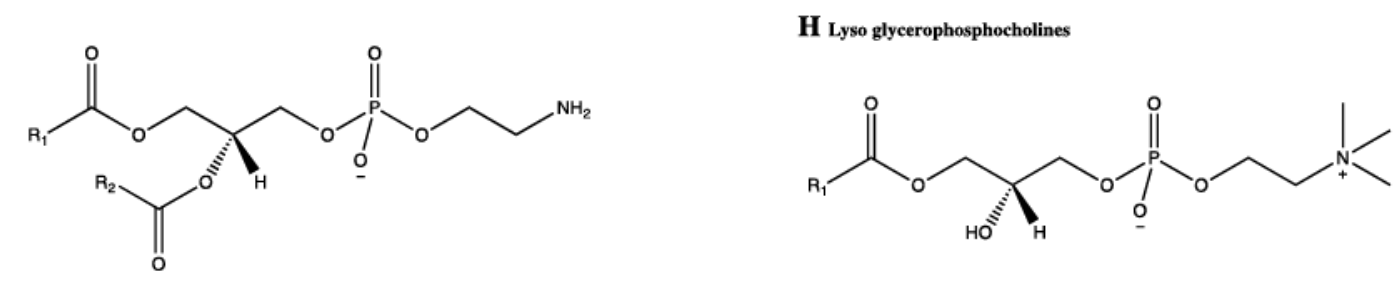

1,2-diacyl-sn-glycero-3-phosphoethanolamine

1-acyl-sn-glycero-3-phosphocholine

Figure 2.1: Representative of the lipid classes focused on this thesis. Adapted from Bou Kahalil et al. ${ }^{1}$ and Eoin Fahy et al. ${ }^{2}$

\subsection{Lipid Extraction and Separation}

Chemicals: LysoPC (13:0) was purchased from Avanti Polar Lipids Inc. (Alabaster, AL, USA) as a powder; sodium acetate, anhydrous, was purchased from Bioshop Canada Inc. (Burlington, ON, Canada); glacial acetic acid was obtained from Anachemia Canada Inc. (Montreal, QC, Canada); absolute ethanol (ethyl alcohol anhydrous) was purchased from Commercial Alcohols Inc. (Brampton, ON, Canada); methanol (Optima, LC/MS grade), 2-propanol (Optima, LC/MS grade), water (Optima, LC/MS grade) and chloroform (HPLC Grade) were purchased from Fisher Scientific (Ottawa, ON, Canada).

Lipid Extraction: Lipids were extracted via a modified ${ }^{3}$ Bligh and Dyer Method. ${ }^{4}$ Tissue samples were removed from a $-80^{\circ} \mathrm{C}$ freezer and placed on ice. Samples were cut using a scalpel from a dissection kit. 3 pieces, each approximately $1 \mathrm{mg}$ (weighed out) were taken from each liver and placed in a disposable $5 \mathrm{~mL}$ test tube. A solution consisting of $2 \%$ acetic acid in $\mathrm{MeOH}$ was made daily $(\mathrm{AcMeOH}) .1 \mathrm{~mL}$ of $2 \% \mathrm{AcMeOH}$ was added to each test tube. Each sample was sonicated on ice using a 130-watt Vibracell ${ }^{\mathrm{TM}}$ sonicator 
(model: VCX130) with a 1/4" (6 mm) probe (Sonics \& Materials Inc., Newtown, CT, USA). Samples were sonicated with the following settings: Time: 1 minute 30 seconds, Pulse On: 20 seconds, Pulse Off: 10 seconds, Amplitude: $20 \%$. A blank and spiked test tubes were also created. Neither the blank nor spiked samples contained any tissue. $42 \mu \mathrm{L}$ of $10 \mu \mathrm{M}$ lyso-phosphatidylcholine (LPC) C13:0 was spiked into each test tube except for the blank. LPC C13:0 was selected since it was not natively present in sample of interest. The samples were transferred to $10 \mathrm{~mL}$ glass Kimble tube ("extraction tube") containing $3.2 \mathrm{~mL}$ of filtered $0.1 \mathrm{M}$ sodium acetate. $0.1 \mathrm{M}$ sodium acetate solution was filtered via suction filtration system with a $0.45 \mu \mathrm{M}$ filter pad (Celltreat Scientific Products, Pepperell, MA, USA). Test tubes were rinsed 3 times with $1 \mathrm{~mL}$ of $2 \% \mathrm{AcMeOH}$, each rinsing was transferred to the appropriate extraction tube (total volume of $2 \% \mathrm{AcMeOH}$ is $4 \mathrm{~mL}$ ). 3.8 $\mathrm{mL}$ of chloroform $\left(\mathrm{CHCl}_{3}\right)$ was added to extraction tubes. The extraction tubes were inverted $5 X$ in the fumehood and $3 X$ right before placing into a centrifuge. Samples were centrifuged for 2 mins at $2000 \mathrm{rpm}$ and $4^{\circ} \mathrm{C}$. The bottom phase was collected via Pasteur pipette and transferred to a clean $10 \mathrm{~mL}$ glass Kimble tube ("collection tube"). Extraction of each sample was repeated three more times with $2 \mathrm{~mL}$ of chloroform each time; all bottom phases were retained in the same collection tube. A constant stream of nitrogen gas was used to evaporate the chloroform until residue remained (complete evaporation). Tissue samples were resuspended in $300 \mu \mathrm{L}$ of $\mathrm{EtOH}$. Resuspended samples were flushed with nitrogen gas. The samples were incubated at $30^{\circ} \mathrm{C}$ for 10 minutes using a MultiTherm shaker (Benchmark Scientific Inc., Edison, NJ, USA) to dissolve lipids and centrifuged for $1 \mathrm{~min}$ at $2000 \mathrm{rpm}$ and $4^{\circ} \mathrm{C}$. Sample solution was transferred over to amber vials with Teflon caps. Amber vials were flushed with a gentle stream of nitrogen gas. The 
samples were diluted $2 \mathrm{X}$ in $\mathrm{MeOH}(50 \mu \mathrm{L}$ of sample $+50 \mu \mathrm{L}$ of $\mathrm{MeOH})$ in a conical autosampler insert (Canadian Life Sciences Inc., Peterborough, ON, Canada). The conical insert was flushed with $\mathrm{N}_{2}(\mathrm{~g})$ and capped in an autosampler vial.

Sample Pooling: Tissue samples were pooled together after the start of the last MS only acquisition mode injection. Pooled samples were used for iterative MS/MS acquisition. 15 $\mu \mathrm{L}$ of each sample in a conical autosampler insert were aliquoted into a single new conical autosampler insert. The pooled sample was mixed via pipetting and was flushed with $\mathrm{N}_{2}$ (g) and capped in an autosampler vial. The autosampler vial was added to the autosampler of an Agilent Quat-pump Infinity II 1260 (Agilent Technologies, CA, USA).

\section{$2.3 \quad$ LC-MS/MS Analysis}

Reversed phase high performance liquid chromatography: An injection volume of $5 \mu \mathrm{L}$ from an autosampler vial with a conical insert (Canadian Life Sciences Inc., Peterborough, ON, Canada) containing $50 \mu \mathrm{L}$ of extracted lipids in $\mathrm{EtOH}$ and $50 \mu \mathrm{L}$ of $\mathrm{MeOH}$ was introduced to the autosampler of an Agilent Quat-pump Infinity II 1260 (Agilent Technologies, CA, USA). Lipids were separated using a reversed-phase C18 column (Agilent's InfinityLab Porocell 120 column, EC-C18, 2.1 x 100 mm, 2.7-micron). The Agilent 1260 Infinity II Multicolumn Thermostat was set to $45^{\circ} \mathrm{C}$ and a binary gradient program (gradient program A) consisting of $50 \% \mathrm{MeOH}, 50 \% \mathrm{H}_{2} \mathrm{O}$ with $10 \mathrm{mM} \mathrm{NH}_{4} \mathrm{HCO}_{2}$ (mobile phase A) and $75 \%$ IPA, $25 \% \mathrm{MeOH}$ with $10 \mathrm{mM} \mathrm{NH}_{4} \mathrm{HCO}_{2}$ (mobile phase B). The HPLC gradient began at $80 \% \mathrm{~A}, 20 \% \mathrm{~B}$ at a flow rate of $0.400 \mathrm{~mL} / \mathrm{min}$ and increased to $100 \%$ B over 37.1 minutes; the run ended with $100 \%$ solvent B at 44 minutes. 


\begin{tabular}{|c|c|c|c|c|c|c|c|}
\hline Time [min] & ' & $\mathrm{A}[\%]$ & $\mathrm{B}[\%]$ & $\mathrm{C}[\%]$ & $\mathrm{D}[\%]$ & $\begin{array}{l}\text { Flow } \\
\text { [mL/min] }\end{array}$ & $\begin{array}{l}\text { Max. Pressure } \\
\text { Limit [bar] }\end{array}$ \\
\hline & 0.00 & 80.0 & 20.0 & 0.0 & 0.0 & 0.400 & 600.00 \\
\hline & 0.35 & 80.0 & 20.0 & 0.0 & 0.0 & $-\cdots$ & $\cdots$ \\
\hline & 0.40 & 68.0 & 32.0 & 0,0 & 0.0 & - & - \\
\hline & 9.60 & 56.0 & 44.0 & 0.0 & 0.0 & $\ldots$ & $\ldots$ \\
\hline & 9.70 & 35.0 & 65.0 & 0.0 & 0.0 & $\cdots$ & $\ldots$ \\
\hline & 11.50 & 35.0 & 65.0 & 0.0 & 0.0 & $\cdots$ & $\cdots$ \\
\hline & 26.80 & 18.0 & 82.0 & 0.0 & 0.0 & $\ldots$ & $\ldots$ \\
\hline & 27.30 & 13.0 & 87.0 & 0.0 & 0.0 & $\cdots$ & $\ldots$ \\
\hline & 37.00 & 40 & 96.0 & 0.0 & 0.0 & $\cdots$ & $\cdots$ \\
\hline & 37.10 & 0.0 & 100.0 & 0.0 & 0.0 & $\ldots$ & $\ldots$ \\
\hline
\end{tabular}

Figure 2.2: A binary gradient program (gradient program $\mathrm{A}$ ) with a constant flow rate $(0.4$ $\mathrm{mL} / \mathrm{min}$ ) for lipid elution was used. Solvent A consisted of $50 \% \mathrm{MeOH}, 50 \% \mathrm{H}_{2} \mathrm{O}$ with 10 $\mathrm{mM} \mathrm{NH} \mathrm{HCO}_{2}$ and solvent $\mathrm{B}$ consisted of $75 \%$ IPA, $25 \% \mathrm{MeOH}$ with $10 \mathrm{mM} \mathrm{NH}_{4} \mathrm{HCO}_{2}$.

After 10 analyses using MS acquisition mode, an internal standard analysis was conducted $(0.7 \mu \mathrm{M}$ in $50 \mu \mathrm{L}$ of $\mathrm{EtOH}+50 \mu \mathrm{L}$ of $\mathrm{MeOH})$. In addition, an internal standard run was conducted after the sixth iterative sample injection. Two full blank runs were introduced at the beginning of each "set" and one at the end of each "set" to further clean the overall system and ensure no lipids were carried over.

HLPC-MS/MS: The HPLC eluent was introduced to an Agilent 6546 LC-QTOF using a Dual Agilent Jet Stream electrospray ionization source (Dual AJS ESI) with the following parameters: positive polarity mass range of $\mathrm{m} / \mathrm{z} 100-1700$; gas temperature, $200^{\circ} \mathrm{C}$; drying gas $10 \mathrm{~L} / \mathrm{min}$; nebulizer, $50 \mathrm{psi}$; sheath gas temperature, $300^{\circ} \mathrm{C}$; sheath gas flow, $12 \mathrm{~L} / \mathrm{min}$; VCap, $3500 \mathrm{~V}$; nozzle voltage, $0 \mathrm{~V}$; fragmentor, $150 \mathrm{~V}$; skimmer, $65 \mathrm{~V}$; and oct $1 \mathrm{RF}$ Vpp, 750 V. For MS/MS iterative acquisition mode, the Agilent 6546 LC/Q-TOF was operated using the following parameters: Positive polarity $\mathrm{MS}^{2}$; auto mass range ( $\mathrm{m} / \mathrm{z} 40$ 1700); narrow isolation width; static exclusion range, $\mathrm{m} / \mathrm{z} 40-150$; iterative, $\pm 20 \mathrm{ppm}, \pm$ 
0.15 retention time tolerance; collision energy. $28 \mathrm{eV}$; all other MS/MS parameters are

the same as positive polarity MS. In addition, iterative MS/MS acquisition mode was used

on 1 blank injection and 6 sample injections. The Agilent 6546 LC-QTOF was tuned using

\section{an Agilent tune mix.}

\begin{tabular}{|c|c|c|c|}
\hline & Sample Name & Sample Position & Method \\
\hline 1 & Dec2_StartBlank1 & P1-A1 & D:MassHunterMethods:FrankYNov24WJosh_LipA\&PW_Custom4_Pos_MSMS.m \\
\hline 2 & Dec2_StartBlank2 & P1-A1 & 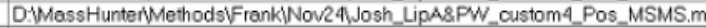 \\
\hline 3 & Dec2_RLE_x2dilution_Control2_Extraction2_Injection2 & P1-A3 & 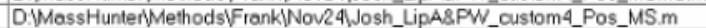 \\
\hline 4 & Dec2_RLE_x2dilution_Control2_Extraction3_Injection2 & P1-A4 & D.MassHunterMethodsiFrankiNov24)Josh_LipA\&PW_custom4_Pos_MS.m \\
\hline 5 & Dec2_RLEX2dilution_MidDose3_Extraction2_Injection2 & P1-A9 & D.MassHunter'MethodsiFrankiNov24 Wosh_LipA\&PW_custom4_Pos_MS.m \\
\hline 6 & Dec2_RLE_x2dilution_MidDose3_Extraction1_Injection1 & $\mathrm{P} 1-\mathrm{A} 8$ & D.WossHunterWethodsVFrankWNov24 Wosh_LipASPW_custom4_Pos_MS.m \\
\hline 7 & Dec2_RLE_x2dilution_Control2_Extraction2_Injection1 & P1-A3 & D.MassHunterMethodsIFrank/Nov24WJosh_LipA\&PW_custom4_Pos_MS.m \\
\hline 8 & Dec2_RLE X2dilution_LowDose1_Extraction3_Injection3 & P1-A7 & D:Mass HunterMethodsiFrankiNov24 Josh_LipA\&PW_Custom4_Pos_MS.m \\
\hline 9 & Dec2_RLE_x2dilution_Control2_Extraction2_Injection3 & P1-A3 & DiMassHunterWhethodsiFrank'Nov24 Wosh_LipASPWW_Custom4_Pos_MSm \\
\hline 10 & Dec2_RLE_x2dilution_HighDose5_Extraction3_Injection2 & P1-B2 & D:MassHunter(MethodsiFrank)Nov24\}Josh_LipA\&PW_custom4_Pos_MS.m \\
\hline 11 & Dec2 RLE X2dilution_HighDose5_Extraction2_Iniection3 & P1-B1 & DUMass HunterMethodsiFrankiNov24Wosh_LipA\&PW_custom4_Pos_MS.m \\
\hline 12 & Dec2_RLE_x2dilution_LowDose1_Extraction2_Injection2 & P1-A6 & 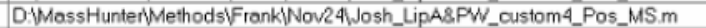 \\
\hline 13 & Dec2_LPC_Diluted_Not_extracted1 & P1-F1 & D:MassHunter'MethodsIFrank'Nov24)Josh_LipA\&PW_custom4_Pos_MS.m \\
\hline 14 & Dec2_RLE_x2dilution_Control2_Extraction1_Injection1 & P1-A2 & D:MassHunterMethodsiFrankiNov24Wosh_LipA\&_PW_custom4_Pos_MS.m \\
\hline 15 & Dec2_RLE_x2dilution_HighDose5_Extraction3_Injection3 & $\mathrm{P} 1-\mathrm{B} 2$ & DWMassHunterWethodsIFrankiNov24 Wosh_LipA8PW_custom4_Pos_MSm \\
\hline 16 & Dec2_RLE_x2dilution_HighDose5_Extraction1_Injection1 & P1-A11 & D:MassHunter|MethodsiFrankiNov24Wosh_LipA\&PW_Custom4_Pos_MS.m \\
\hline 17 & Dec2_RLE x2 dilution_MidDose3_Extraction1_Injection2 & P1-A8 & D:Mass Hunter'MethodsIFrankiNov24 Josh_LipA\&PW_custom4_Pos_MS.m \\
\hline 18 & Dec2_RLE_x2dilution_HighDose5_Extraction2_Injection1 & P1-B1 & D.MossHunterWethodsVFrankiNov24Wosh_LipASPPW_custom4_Pos_MSm \\
\hline 19 & Dec2_RLE_x2dilution_MidDose3_Exaraction2_Injection1 & P1-A9 & D.MassHunter|MethodsiFrankiNov24Wosh_LipA\&PW_custom4_Pos_MS.m \\
\hline 20 & Dec2_RLE_x2dilution_Control2_Extraction3_Injection1 & P1-A4 & 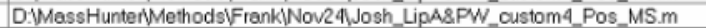 \\
\hline 21 & Dec2_RLE_x2dilution_HighDose5_Extraction3_Injection1 & P1-B2 & DUMoss HunterWMethodsVFrank'Nov24WJosh_LipA\&PW_custom4_Pos_MS.m \\
\hline 22 & Dec2_RLE_x2dilution_HighDose5_Extraction1_Injection3 & P1-A11 & D.MassHunterMethodsiFrankiNov24WJosh_LipA\&PW_Custom4_Pos_MS.m \\
\hline 23 & Dec2_RLE_x2dilution_Control2_Extraction1_Injection 3 & $\mathrm{P} 1-\mathrm{A} 2$ & 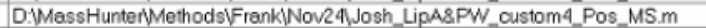 \\
\hline 24 & Dec2_LPC_Diluted_Not_extracted2 & P1-F1 & D.MassHunterWethodsVFrankWNov24Wosh_LipASPWW_custom4_Pos_MS.m \\
\hline 25 & Dec2_RLE_x2dilution_Control2_Extraction1_Injection2 & P1-A2 & D:MassHunter(MethodsiFrankiNov24)Josh_LipA\&PW_Custom4_Pos_MS.m \\
\hline 26 & Dec2 RLE_x dilution_LowDose1_Extraction1_Injection1 & P1-A5 & D.MassHunteriMethodsiFrankiNov24 Josh_LipA\&_PW_custom4_Pos_MS.m \\
\hline 27 & Dec2_RLE_x2dilution_MidDose3_Exdraction1_Injection3 & $\mathrm{P} 1-\mathrm{A} 8$ & 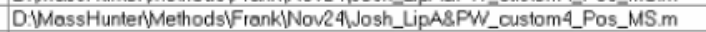 \\
\hline 28 & Dec2_RLE_x2dilution_LowDose1_Extraction1_Injection3 & P1-A5 & D.MassHunterMethodsIFrank/Nov24Wosh_LipA\&PW_Custom4_Pos_MS.m \\
\hline 29 & Dec2_RLE_x dilution_MidDose3_Extraction3_Injection2 & $\mathrm{P} 1-\mathrm{A} 10$ & D.MassHunterMethodsiFrankiNov24 Josh_LipA\&.PW_custom4_Pos_MS.m \\
\hline 30 & Dec2_RLE_x2dilution_LowDose1_Extraction3_Injection2 & P1-A7 & 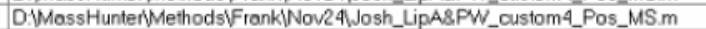 \\
\hline 31 & Dec2_RLE_x2dilution_LowDose1_Extraction2_Injection1 & P1-A6 & D.MassHunter|MethodsiFrankiNov24(Josh_LipA8PW_Custom4_Pos_MS.m \\
\hline 32 & Dec2_RLEX2dilution_LowDose1_Extraction1_Injection2 & P1-A5 & D.MassHunterlMethodsiFrankiNov24 Josh_LipA\&PW_custom4_Pos_MS.m \\
\hline 33 & Dec2_RLE_x2dilution_MidDose3_Extraction2_Injection3 & P1-A9 & 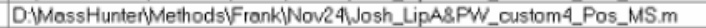 \\
\hline 34 & Dec2_LPC_Diluted_Not_extracted 3 & P1-F1 & D.MassHunter|MethodsVFrank/Nov24 Josh_LipA\&PWW_Custom4_Pos_MS.m \\
\hline 35 & Dec2_RLE_x2dilution_MidDose3_Extraction3_Injection1 & P1-A10 & D:MassHunterMethodsiFrankiNov24Wosh_LipA\&PW_Custom4_Pos_MS.m \\
\hline 36 & Dec2_RLE_x2dilution_HighDose5_Extraction1_Injection2 & P1-A11 & D.Mass HunteriWethodsfFrankiNov24 Wosh_LipA\&PWW_custom4_Pos_MS.m \\
\hline 37 & Dec2_RLE_x2dilution_MidDose3_Extraction3_Injection3 & P1-A10 & D:MassHunterMethodsiFrankiNov24(Josh_LipABPW_Custom4_Pos_MS.m \\
\hline 38 & Dec2_RLE x 2dilution_Control2_Extraction3_Injection 3 & P1-A4 & D.MassHunterMethodsIFrankiNov24WJosh_LipA\&PW custom4_Pos_MS.m \\
\hline 39 & Dec2_RLE_x2dilution_LowDose1_Extraction3_Injection1 & P1-A? & D.MassHunterWethodsFrankiNov24Wosh_LipA\&PW_Custom4_Pos_MS.m \\
\hline 40 & Dec2_RLE_x2dilution_HighDose5_Extraction2_Injection2 & P1-B1 & DiMassHunterWethodsiFrankiNov24 Josh_LipA\&PW_Custom4_Pos_MS.m \\
\hline 41 & Dec2_RLE_x2dilution_LowDose1_Extraction2_Injection3 & P1-A6 & 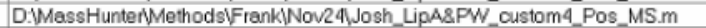 \\
\hline 42 & Dec2_LPC_Diluted_Not_extracted4 & P1-F1 & D.WassHunterMethods\}FrankWNov24Wosh_LipA\&PW_custom4_Pos_MS.m \\
\hline 43 & Dec2_MatráBlank_tterative_BackgroundExtraction & P1-B3 & D.MassHunterMethodsIFrankWNov24WJosh_LipA8PW_Custom4_Pos_MSMS,m \\
\hline 44 & Dec2_Pooled_RLE_Iterative_1 & P1-B4 & D.WMassHunter'MethodsiFrank|Nov24Wosh_LipA\&PW_Custom4_Pos_MSMS.m \\
\hline 45 & Dec2_Pooled_RLE_lterotive_2 & P1-B4 & 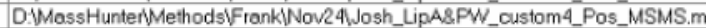 \\
\hline 46 & Dec2_Pooled_RLE_lierative_3 & P1-B4 & D:MassHunterWMethods)FrankWNov24 Josh_LipA8PW_Custom4_Pos_MSMS,m \\
\hline 47 & Dec2_Pooled_RLE_Iterative_4 & P1-B4 & D.MassHunterMethodsIFrankINov24Wosh_LipA\&PW_Custom4_Pos_MSMS.m \\
\hline 48 & Dec2_Pooled_RLE_titerotive_5 & P1-B4 & D.Mass Hunter'MethodsiFrankWNov24Wosh_LipASPPW_custom4_Pos_MSMS.m \\
\hline 49 & Dec2_Pooled_RLE_literative_6 & P1-B4 & D.MassHunterMethodsIFrankWNov24Wosh_LipA8PW_Custom4_Pos_MSMS.m \\
\hline 50 & Dec2_LPC_Diluted_Not_extracted5 & P1-F1 & D.WassHunter|MethodsiFrank|Nov24)Josh_LipA\&PW_Custom4_Pos_MS.m \\
\hline 51 & Dec2_MatroxSpiked_LPC_Extracted_Istd_1 & P1-85 & 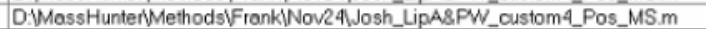 \\
\hline 52 & Dec2_MatrixSpiked_LPC_Extracted_Istd_2 & P1-B5 & 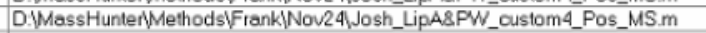 \\
\hline 53 & Dec2_MatroSpiked_LPC_Extracted_Istd_3 & P1-B5 & D:MassHunterMethodsiFrankiNov24 Josh_LipA\&PW_custom4_Pos_MS.m \\
\hline 54 & Dec2_MidBlank1 & P1-A1 & D.MassiHunterMethodsiFrankiNov24 Josh_LipAB.PW_custom4_Pos_MSMS.m \\
\hline 55 & Dec2_BLE_x5dilution_RoomTemp & P1-B6 & D.MassHunter|MethodsWFrankINov24 Josh_LipA\&PW_Custom4_Pos_MSMS.m \\
\hline 56 & Dec2_BLE_x5dilution_FreezerTemp & P1-B7 & D.MassHunterMethodsiFrankiNov24)Josh_LipA\&PW_Custom4_Pos_MSMS.m \\
\hline 57 & Dec4_End-StantBlonk & P1-A1 & DWMassHunter'MethodsiFrankiNov24 Wosh_LipA\&PW_Custom4_Pos_MSMS.m \\
\hline
\end{tabular}

Figure 2.3: An excerpt from the worklist with internal standard quality control (QC) injections. 


\subsection{Post-Data Acquisition (Agilent Software)}

Following data acquisition, a suite of different Agilent programs was used for data analysis. Figures 2.4 and 2.5 illustrates the overall lipidomics workflow performed in this thesis. Iterative MS/MS acquisition data in positive polarity was analyzed using Lipid Annotator. ${ }^{5}$ An exported file was appended into MassHunter Personal Compound Database and Library (PCDL) Manager software. This database was used to perform a batch target feature extraction from MS level acquired data in Profinder based on both retention time and accurate mass. Data were exported into Microsoft Excel where manual normalization was performed before inputting into RStudio for statistical interpretation.

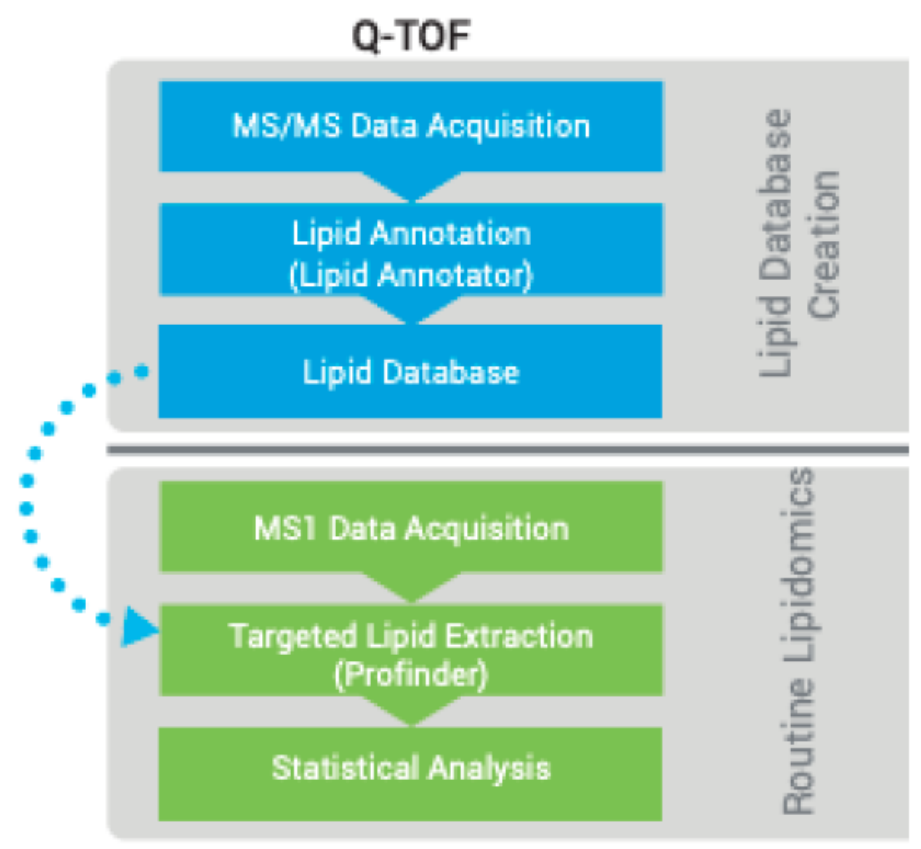

Figure 2.4: A general untargeted workflow for lipid analysis utilizing Agilent's Lipid Annotator. Figure adapted from Agilent Technologies. ${ }^{6}$ 


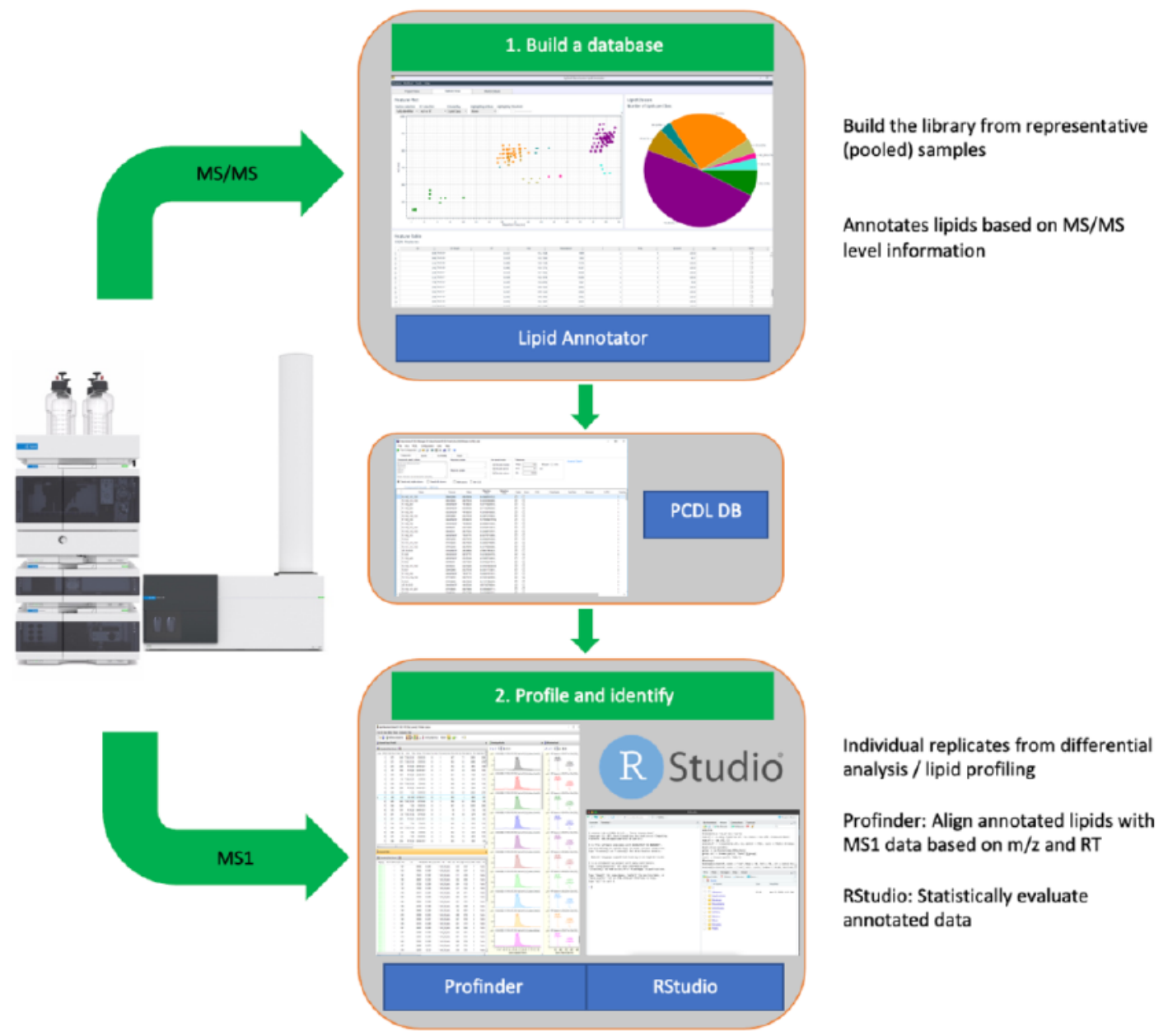

Figure 2.5: A general untargeted workflow for lipid analysis using a suite of Agilent software and RStudio. Figure adapted from Klein, C. \& Sartain, M. ${ }^{6}$

\subsubsection{MassHunter Qualitative Analysis}

MassHunter Qualitative Analysis (Version 10.0) was used to provide internal standard quality control. An extracted ion chromatogram (EIC) of 454.29 Da was performed to determine retention time and intensity. MassHunter Qualitative Analysis was also used while performing quality assurance throughout post-data acquisition for presence of false positives or anomalies. 


\subsubsection{MassHunter Lipid Annotator}

MassHunter Lipid Annotator (Version 1.0) was used for post data acquisition of MS/MS injections. Lipid Annotator uses an expanded LipidBlast ${ }^{7}$ lipid library for in silico lipid annotation. ${ }^{5}$ Briefly, the algorithm for Lipid Annotator consists of finding features associated in MS/MS scans, annotates features for possible lipids, calculates the abundances of each constituent based on both in silico and experimental spectra and finally filters the annotated feature by exact mass, isotope, and MS/MS match score. A combination of probability density, Bayesian scoring, and non-negative least square fit allows Lipid Annotator to successfully annotate lipids for both in vivo and in vitro studies. ${ }^{5}$

Data files from the MS/MS iterative acquisition mode of each set were compiled into a single Lipid Annotator project. The default method was used to process the files which consisted of the following parameters in Figure 2.6 - 2.8. Once lipid annotation was complete, data was exported in both .csv and PCDL formats for further downstream analysis.

Feature Finding Parameters
Feature Filter
$\square$ Q-Score $\geq 30.0$
$\square$ Abundance $\geq \square$
$\square$ Restrict RT to $\square$ min.
$\square$ Restrict $\mathrm{m} / 2$ to $\mathrm{m} / 2$

Figure 2.6: Lipid Annotator's feature finding Q-score parameter was set to Q-score lower than 30.0. Q-score is defined as the chromatographic peak quality. Any feature that form a very poor chromatographic peak was filtered out of the feature list. 


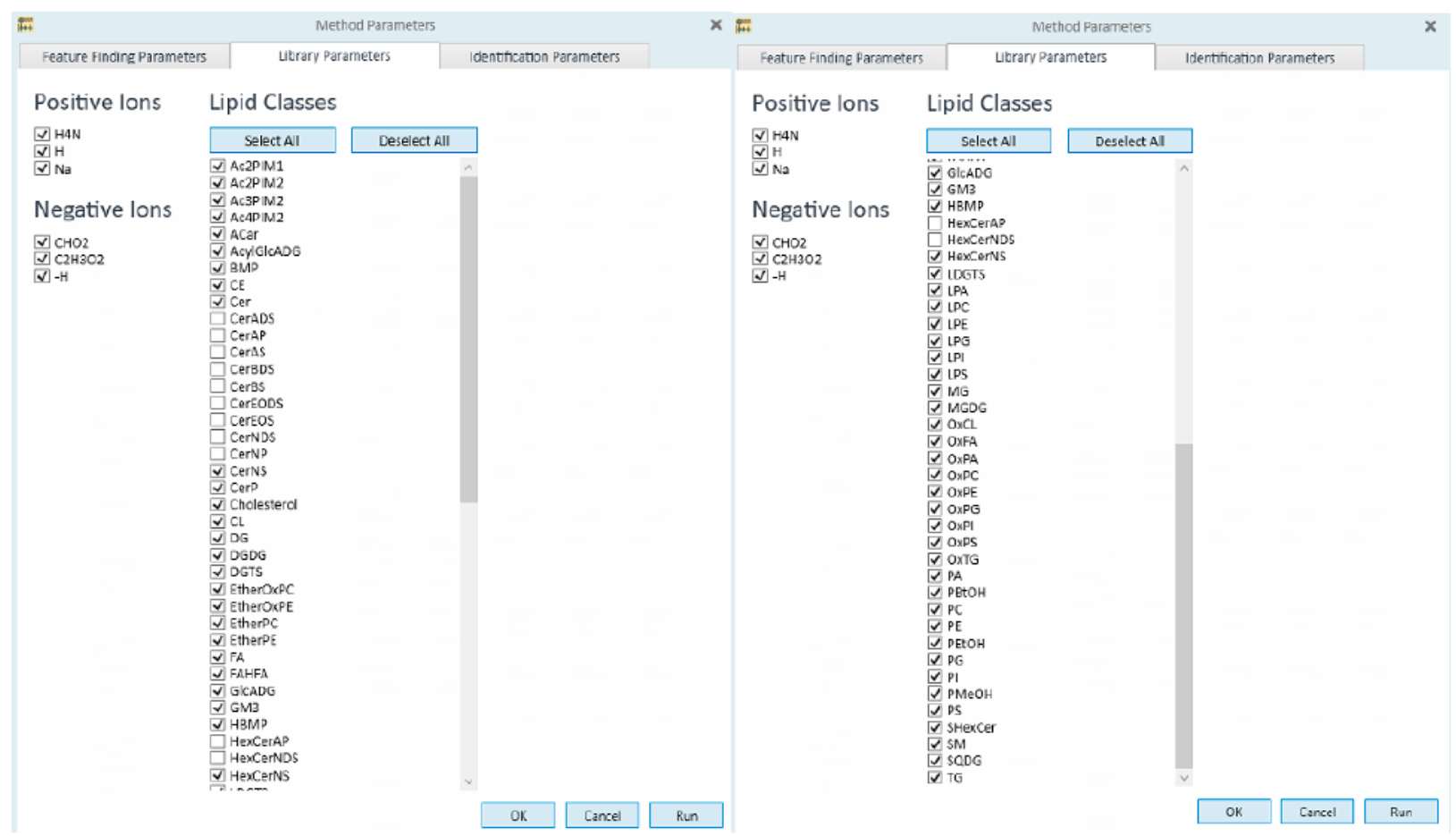

Figure 2.7: Both polarities were selected including all common adducts. The following lipid classes were selected for annotation.

\begin{tabular}{|c|c|c|}
\hline 4 & Method Para & \\
\hline Feature Finding Parameters & Library Parameters & Identification Parameters \\
\hline
\end{tabular}

\section{Sum Composition Level}

Mass Threshold

$\checkmark$ Mass deviation $\leq 10.0$ ppm

Multiple Lipid IDs for Same Feature

$\checkmark$ Report top candidate only

Score Threshold

$\checkmark$ Fragment score $\geq 30$

$\checkmark$ Total score $\geq 60$

\section{Constituent Level}

Report dominant constituent if relative abundance differential $\geq 10 \%$

Figure 2.8: Identification parameters were set in Lipid Annotator to reduce the amount of falsely identified lipids. 


\subsubsection{Personal Compound Database \& Library (PCDL) Manager}

Personal Compound Database \& Library (PCDL) Manager (version B.08.00) was used to manage lipidomics data from Lipid Annotator. Each exported data from Lipid Annotator in PCDL format was edited to select cation (since positive polarity was selected). Parameters such as tolerances were transferred over from the default method selected in Lipid Annotator. Figure 2.9 illustrates a PCDL database that was used for further downstream analysis.

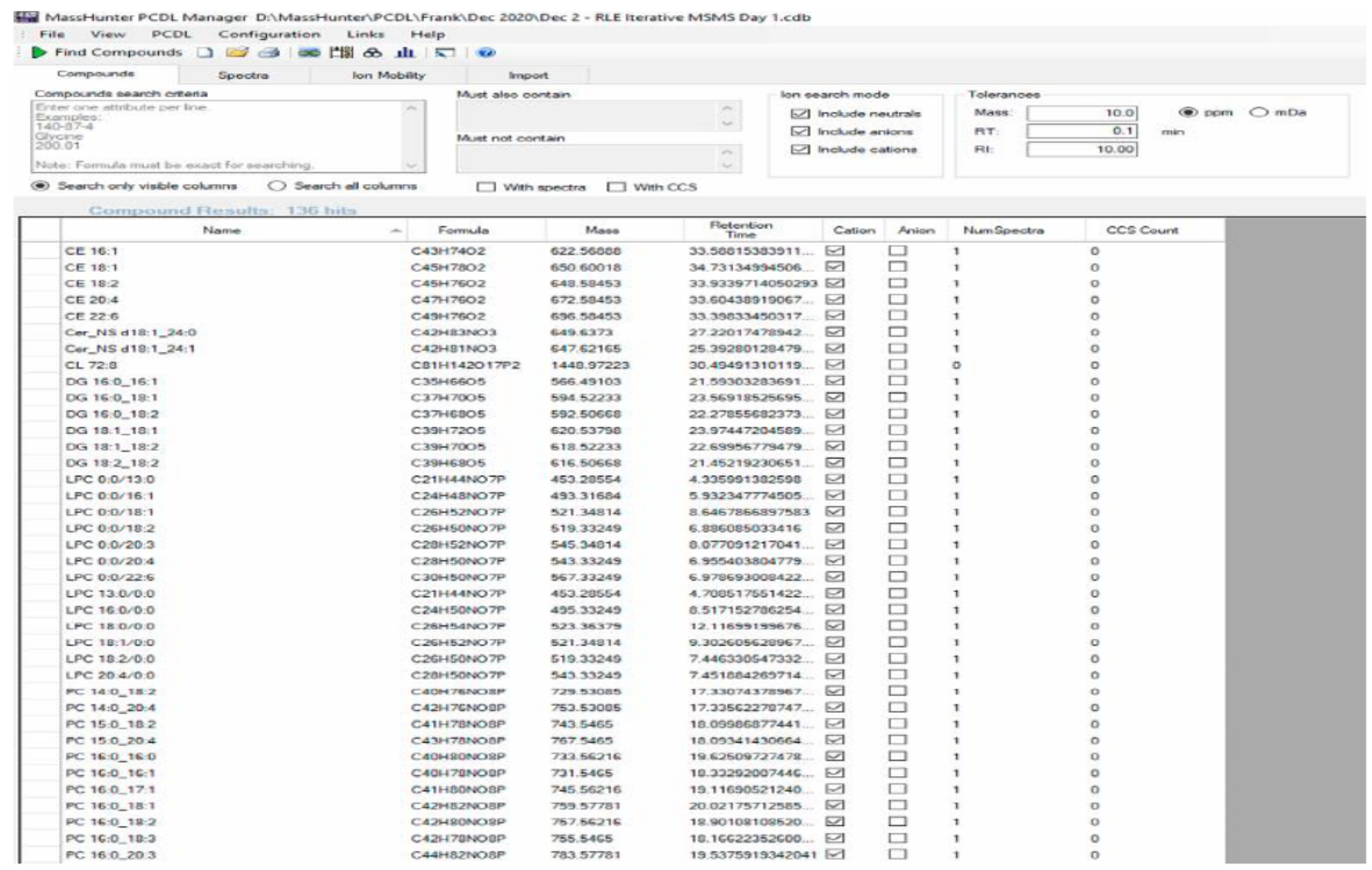

Figure 2.9: An example of a lipidomics database in PCDL.

\subsubsection{Profinder}

Profinder (Version 10.0) was used to perform a batch targeted feature extraction from MS1 level data acquired separately. Combining both the database created in PCDL from MS/MS level data and MS1 level data, lipid species were identified based on accurate 
mass and retention time. Profinder was also used to align retention times from MS1 level data to ensure correct peaks were extracted. In addition, Profinder consolidated adducts into a single entry, reducing redundancies.

Parameters for a batch targeted feature extraction were as followed: the appropriate PCDL database was selected; maximum number of matches, 1; values to match, Mass and retention time (retention time required); positive ions, $+\mathrm{H},+\mathrm{Na},+\mathrm{NH}_{4}$; charge state range, 1 ; isotope model, common organic (no halogens); match tolerances, mass \pm 20 $\mathrm{ppm}$, retention time \pm 0.15 mins; possible $\mathrm{m} / \mathrm{z}$ symmetric $(\mathrm{ppm}) \pm 35$; limit EIC extraction range; expected retention time, symmetric \pm 0.75 mins; contribution to overall score, mass score $=100.00$, isotope abundance score $=60.00$, isotope spacing score $=50.00$, retention time score $=100.00 ;$ expected data variation, $\mathrm{MS}$ mass $=2.0 \mathrm{mDa} \pm 5.6 \mathrm{ppm}$, $\mathrm{MS} / \mathrm{MS}$ mass $=5.0 \mathrm{mDa} \pm 7.5 \mathrm{ppm}$, retention time $=0.115 \mathrm{~min}$; height filters, and absolute height $\geq 1000$ counts. Manual integrations were performed as needed and data was saved and exported in .csv format for statistical analysis.

\subsection{Downstream Workflow \& RStudio}

After data was exported, normalization of each lipid species was performed by dividing the area of each lipid found by the area of the spiked internal standard. Further normalization to the wet tissue weight was also calculated using Microsoft Excel by dividing the normalize lipid by the weight of the wet tissue. Empty cells were filled by determining the lowest normalized intensity and multiplying it by an arbitrary value, in this case $36 \%(0.36)$, to give a low, insignificant value while maintaining a non-zero realistic value. Datasets were amalgamated and inputted into RStudio (Version 4.0.3) to produce various statistical plots and figures. In addition, scripts were written in RStudio to calculate 
fold change and statistically significant changes. Fold change was calculated by dividing the averaged relative intensity of each lipid by its counterpart in the lower dosage treatment. For example, fold change of PC 16:0/18:1 between low and high dose was determined by dividing the averaged relative intensity found in the high dose by the averaged relative intensity found in the low dose. Statistically significant changes were assessed using student's t-test $(p$-value $(\alpha)=0.05)$ followed by a $p$-value correction $(\alpha=$ $0.01)$ for false discovery rates (FDR).

\subsection{References}

(1) Bou Khalil, M.; Hou, W.; Zhou, H.; Elisma, F.; Swayne, L. A.; Blanchard, A. P.; Yao, Z.; Bennett, S. A. L.; Figeys, D. Lipidomics Era: Accomplishments and Challenges: LIPIDOMICS ERA: ACCOMPLISHMENTS AND CHALLENGES. Mass Spectrom. Rev. 2010, 29 (6), 877-929. https://doi.org/10.1002/mas.20294.

(2) Fahy, E.; Subramaniam, S.; Brown, H. A.; Glass, C. K.; Merrill, A. H.; Murphy, R. C.; Raetz, C. R. H.; Russell, D. W.; Seyama, Y.; Shaw, W.; Shimizu, T.; Spener, F.; van Meer, G.; VanNieuwenhze, M. S.; White, S. H.; Witztum, J. L.; Dennis, E. A. A Comprehensive Classification System for Lipids. J. Lipid Res. 2005, 46 (5), 839861. https://doi.org/10.1194/jlr.E400004-JLR200.

(3) Bonin, F.; Ryan, S. D.; Migahed, L.; Mo, F.; Lallier, J.; Franks, D. J.; Arai, H.; Bennett, S. A. L. Anti-Apoptotic Actions of the Platelet-Activating Factor Acetylhydrolase I A2 Catalytic Subunit. J. Biol. Chem. 2004, 279 (50), 5242552436. https://doi.org/10.1074/jbc.M410967200.

(4) Bligh, E. G.; Dyer, W. J. A RAPID METHOD OF TOTAL LIPID EXTRACTION AND PURIFICATION. Can J Biochem Physiol 1959, 37 (8), 911-917.

(5) Koelmel, J. P.; Li, X.; Stow, S. M.; Sartain, M. J.; Murali, A.; Kemperman, R.; Tsugawa, H.; Takahashi, M.; Vasiliou, V.; Bowden, J. A.; Yost, R. A.; Garrett, T. J.; Kitagawa, N. Lipid Annotator: Towards Accurate Annotation in Non-Targeted Liquid Chromatography High-Resolution Tandem Mass Spectrometry (LC-HRMS/MS) Lipidomics Using a Rapid and User-Friendly Software. Metabolites 2020, 10 (101), 20. https://doi.org/10.3390/metabo10030101.

(6) Klein, C.; Sartain, M. J. Lipids Greatest Hits - Featuring the Agilent 6546 LC/Q-TOF and Lipid Annotator - Special Breakfast Performance, 2019. 
(7) Kind, T.; Liu, K.-H.; Lee, D. Y.; DeFelice, B.; Meissen, J. K.; Fiehn, O. LipidBlast in Silico Tandem Mass Spectrometry Database for Lipid Identification. Nat. Methods 2013, 10 (8), 755-758. https://doi.org/10.1038/nmeth.2551. 


\section{Chapter 3: Optimization and Developmental Experiments Prior to Analysis of Rat}

\section{Liver Samples using Bovine and Control Rat Liver Tissues}

\subsection{Introduction}

Since the introduction of lipidomics in $2003^{1}$, there has been an increase of advancements made to improve analyses of lipids from complex biological systems. ${ }^{2}$ With the incorporation of both liquid chromatography and mass spectrometry, the popularity of LC-MS(/MS) based methods grew due to its reliable identification, better separation, and reduction of suppression effects. ${ }^{2-4}$ In lipidomics studies that utilize HPLC, reversedphase and normal phase are most frequently used however, as described in Chapter 1.3, the focus will specifically be on reversed-phase HPLC. In general, reversed-phase HPLC itself offers great separation efficiencies for both high throughput and comprehensive MSbased lipidomics analysis. ${ }^{4}$ This is especially powerful in untargeted lipidomics since the goal is generally to obtain efficient, reliable, and comprehensive identification and quantification of lipids. ${ }^{3}$ However, to ensure reproducibility and robustness of any results, method development and optimization are still necessary.

In this chapter, key aspects of the optimization were performed using lipid extracts from bovine liver and control rat liver tissues. Bovine liver was used as there was a limited amount of control rat livers available. However, to ensure that parameters are applicable for lipid extracts from rat liver, experiments were repeated once with control rat liver tissues. While Chapter 2 outlines the general methodologies used throughout this thesis, this chapter illustrates the modifications based on the performed experiments. These experiments include determining appropriate sample dilutions, developing an iterative methodology, and optimizing an acquisition method. This chapter presents key 
experiments performed on the Agilent LC-QTOF 6546. During optimization of many different lipidomic methods, a variety of QC samples was implemented to ensure the data acquired is of high quality. Many of these QC samples include an internal standard and several blanks. One notable issue with the LC-based lipidomics method is carry-over. To address this, an additional step was incorporated into the gradient program to ensure full elution of lipids from the system, while being time efficient. All these experiments are discussed in further detail in this chapter.

\subsection{Experimental}

Sample Preparation: Bovine liver tissues were obtained from a local grocery store by Prof. Jeffrey Smith. Tissues were transferred from original packaging into Ziplock bags and stored in a $-20^{\circ} \mathrm{C}$ freezer. Control rat liver tissues were obtained from collaborative work with Dr. Baki Sadi at Health Canada, Radiation Protection Bureau. The animal experiment was carried out using male Wistar rats at 9 weeks of age. Each Wistar rat weighed approximately $400 \mathrm{~g}$. The rats were purchased from Charles River Laboratories International Inc. (www.criver.com/products-services/find-model/wistar-igs-rat) Upon arrival, the rats were kept in a metabolic cage for a minimum of 10-14 days prior to the beginning of this experiment to acclimatize to their new surroundings. The rats were kept under a constant temperature of $23 \pm 2^{\circ} \mathrm{C}$. During acclimatization, rats were kept in a 12hour light and 12-hour dark cycle with free access to rodent chow and reverse osmosis water. All work with the rats were conducted within the Biological Research Facility of the Canadian Nuclear Laboratories (CNL), Chalk River, Ontario, Canada in accordance with the Canadian Council on Animal Care (CCAC) guidelines and policies for performing animal experiments and with an Animal Care Committee (ACC) approved animal 
protocol. A group of 5 (control) were injected with $0.3 \mathrm{~mL}$ of $1 \%$ sodium citrate. Rats were kept in the metabolic cages for 9 days with free access to rodent chow and reverse osmosis water. Overall health, weight, food and water intake, and waste output were monitored daily. On day 10 , the rats were euthanized using the CCAC approved method of inhalant anesthetic. Livers were removed from the rats, approximately $1 \mathrm{~g}$ of liver tissue from each rat were stored in $-80^{\circ} \mathrm{C}$. Rat liver tissues were sent to the Radiation Protection Bureau of Health Canada on dry ice for further analysis. Upon arrival, liver tissues were homogenized using a tissue homogenizer and subsampled into multiple tissue homogenates and stored at $-80^{\circ} \mathrm{C}$ for future analysis. One of these subsets were allocated for lipidomics analysis at Carleton University. All tissue samples were kept in Eppendorf tubes. The subset tissue samples were removed from the $-80^{\circ} \mathrm{C}$ freezer and was transported to Carleton University, Carleton Mass Spectrometry Centre on ice. Upon arrival, samples were stored in a $-80^{\circ} \mathrm{C}$ freezer.

Lipid Extraction: See Chapter 2.2. Bovine liver tissues were diluted $5 X(20 \mu \mathrm{L}$ of sample $+80 \mu \mathrm{L}$ of $\mathrm{MeOH}$ and $20 \mu \mathrm{L}$ of sample $+20 \mu \mathrm{L}$ of $\mathrm{EtOH}+80 \mu \mathrm{L}$ of $\mathrm{MeOH}$ ) in 2 conical autosampler inserts (Canadian Life Sciences Inc., Peterborough, ON, Canada). The conical inserts were flushed with $\mathrm{N}_{2}(\mathrm{~g})$ and capped in an autosampler vial.

Reversed phase high performance liquid chromatography: An injection volume of $5 \mu \mathrm{L}$ was introduced to the autosampler of an Agilent Quat-pump Infinity II 1260 (Agilent Technologies, CA, USA). Lipids were separated using a reverse-phase C18 column (Agilent's InfinityLab Porocell 120 column, EC-C18, $2.1 \times 100$ mm, 2.7-micron). The Agilent 1260 Infinity II Multicolumn Thermostat set to $45^{\circ} \mathrm{C}$. Several binary gradient programs were used. One gradient program (gradient program A) consisted of $50 \%$ 
$\mathrm{MeOH}, 50 \% \mathrm{H}_{2} \mathrm{O}$ with $10 \mathrm{mM} \mathrm{NH}_{4} \mathrm{HCO}_{2}$ (mobile phase A) and $75 \%$ IPA, $25 \% \mathrm{MeOH}$ with $10 \mathrm{mM} \mathrm{NH}_{4} \mathrm{HCO}_{2}$ (mobile phase B). The HPLC gradient began at $80 \% \mathrm{~A}, 20 \% \mathrm{~B}$ at a flow rate of $0.400 \mathrm{~mL} / \mathrm{min}$ and increased to $100 \% \mathrm{~B}$ over 37.1 minutes; the run ended with $100 \%$ solvent B at 44 mins; see Figure 2.2. A second gradient program (gradient program B) used consisted of $50 \% \mathrm{MeOH}, 50 \% \mathrm{H}_{2} \mathrm{O}$ with $10 \mathrm{mM} \mathrm{NH}_{4} \mathrm{HCO}_{2}$ (mobile phase A) and $25 \%$ IPA, $75 \% \mathrm{MeOH}$ with $10 \mathrm{mM} \mathrm{NH}_{4} \mathrm{HCO}_{2}$ (mobile phase C). The HPLC gradient began at $100 \% \mathrm{~A}$ at a flow rate of $0.400 \mathrm{~mL} / \mathrm{min}$ and increased to $100 \% \mathrm{C}$ over $11 \mathrm{mins}$; the run ended with $100 \%$ solvent $C$ at 25 mins; see Figure 3.1. A third gradient program (gradient program C) used consisted of $50 \% \mathrm{MeOH}, 50 \% \mathrm{H}_{2} \mathrm{O}$ with $10 \mathrm{mM} \mathrm{NH}_{4} \mathrm{HCO}_{2}$ (mobile phase A), $75 \%$ IPA, $25 \% \mathrm{MeOH}$ with $10 \mathrm{mM} \mathrm{NH}_{4} \mathrm{HCO}_{2}$ (mobile phase B) and $25 \%$ IPA, $75 \%$ $\mathrm{MeOH}$ with $10 \mathrm{mM} \mathrm{NH}_{4} \mathrm{HCO}_{2}$ (mobile phase C). The HPLC gradient began at $100 \% \mathrm{~A}$ at a flow rate of $0.400 \mathrm{~mL} / \mathrm{min}$ and increased to $100 \% \mathrm{C}$ over $11 \mathrm{mins}$. At the $25^{\text {th }}$ minute mark, the gradient switched to $100 \%$ B over 30 seconds; the run ended with $100 \%$ solvent B at 30.5 mins; see Figure 3.2.

\begin{tabular}{|c|c|c|c|c|c|c|c|}
\hline \multirow[b]{2}{*}{ Time [min] } & \multirow[b]{2}{*}{$x$} & \multirow[b]{2}{*}{$A[\%]$} & \multirow[b]{2}{*}{$\mathrm{B}[\%]$} & \multirow[b]{2}{*}{$C[\%]$} & \multirow[b]{2}{*}{$D[\%]$} & \multirow[b]{2}{*}{$\begin{array}{l}\text { Flow } \\
\text { [mUmin] }\end{array}$} & $\square$ function centric view \\
\hline & & & & & & & $\begin{array}{l}\text { Max. Pressure } \\
\text { Limit [bar] }\end{array}$ \\
\hline & 0.00 & 100.0 & 0.0 & 0.0 & 00 & 0.400 & 600.00 \\
\hline & 1.00 & 30.0 & 0.0 & 70.0 & 0.0 & 0.400 & - \\
\hline & 3.50 & 14.0 & 0.0 & 860 & 0.0 & 0.400 & $\ldots$ \\
\hline & 10.00 & 14.0 & 0.0 & 86.0 & 0.0 & 0.400 & - \\
\hline & 11.00 & 0.0 & 0.0 & 100.0 & 0.0 & 0.400 & $\ldots$ \\
\hline & 20.00 & 0.0 & 0.0 & 100.0 & 0.0 & 0.400 & $\ldots$ \\
\hline & 25.00 & 0.0 & 0.0 & 100.0 & 0.0 & 0.400 & $\cdots$ \\
\hline
\end{tabular}

Figure 3.1: Gradient program $B$ with a constant flow rate $(0.4 \mathrm{~mL} / \mathrm{min})$ for lipid elution was used. Solvent $A$ consisted of $50 \% \mathrm{MeOH}, 50 \% \mathrm{H}_{2} \mathrm{O}$ with $10 \mathrm{mM} \mathrm{NH}_{4} \mathrm{HCO}_{2}$ and solvent $\mathrm{C}$ consisted of $25 \%$ IPA, $75 \% \mathrm{MeOH}$ with $10 \mathrm{mM} \mathrm{NH}_{4} \mathrm{HCO}_{2}$. 


\begin{tabular}{|c|c|c|c|c|c|c|c|}
\hline \multirow[b]{2}{*}{ Time [min] } & \multirow[b]{2}{*}{3} & \multirow[b]{2}{*}{$A[\%]$} & \multirow[b]{2}{*}{$\mathrm{B}[\%]$} & \multirow[b]{2}{*}{$C[\%]$} & \multirow[b]{2}{*}{$D[\%]$} & \multirow[b]{2}{*}{$\begin{array}{l}\text { Flow } \\
\text { [mLmin] }\end{array}$} & $\square$ function centric view \\
\hline & & & & & & & $\begin{array}{l}\text { Max Pressure } \\
\text { Limit [bar] }\end{array}$ \\
\hline & 0.00 & 100.0 & 0.0 & 0.0 & 0.0 & 0.400 & 600.00 \\
\hline & 1.00 & 30.0 & 0.0 & 70.0 & 0.0 & 0.400 & - \\
\hline & 3.50 & 14.0 & 0.0 & 86.0 & 0.0 & 0.400 & $\cdots$ \\
\hline & 10.00 & 14.0 & 0.0 & 86.0 & 0.0 & 0.400 & - \\
\hline & 11.00 & 0.0 & 0.0 & 100.0 & 0.0 & 0.400 & $\ldots$ \\
\hline & 20.00 & 0.0 & 0.0 & 100.0 & 0.0 & 0.400 & $\ldots$ \\
\hline & 25.00 & 0.0 & 0.0 & 1000 & 0.0 & 0.400 & $\cdots$ \\
\hline & 25.50 & 0.0 & 100.0 & 0.0 & 00 & $\ldots$ & - \\
\hline
\end{tabular}

Figure 3.2: Gradient program $C$ with a constant flow rate $(0.4 \mathrm{~mL} / \mathrm{min})$ for lipid elution was used. Solvent A consisted of $50 \% \mathrm{MeOH}, 50 \% \mathrm{H}_{2} \mathrm{O}$ with $10 \mathrm{mM} \mathrm{NH} \mathrm{HCO}_{2}$, solvent B consisted of $75 \%$ IPA, $25 \% \mathrm{MeOH}$ with $10 \mathrm{mM} \mathrm{NH}_{4} \mathrm{HCO}_{2}$ and solvent $\mathrm{C}$ consisted of $25 \%$ IPA, $75 \% \mathrm{MeOH}$ with $10 \mathrm{mM} \mathrm{NH}_{4} \mathrm{HCO}_{2}$.

HLPC-MS/MS: See Chapter 2.3

Data Analysis: See Chapter 2.4 and 2.5

\subsection{Results and Discussion}

Before measuring the effects of LDR exposure on Wistar rat liver lipidome, optimization of both the sample preparation and the Agilent LC \& QTOF 6546 were conducted. Method validation of both the LC and QTOF were carried using bovine liver tissues and control rat liver tissues to ensure reproducibility. Once lipid extraction was complete, lipids were analyzed using three different gradient programs described in Chapter 3.2. Analysis of the MS data acquired from the bovine liver tissues yielded a total of 72 lipids, 55 lipids and 55 lipids from gradient programs A, B, and $C$ respectively using Agilent's Lipid Annotator. From gradient program A, 3 Cer_NS, 4 DG, 13 LPC, 24 PC, 2 $\mathrm{PE}, 4 \mathrm{SM}$, and $22 \mathrm{TG}$ were fully identified, illustrated in Figure 3.3. From gradient program B, 4 Cer_NS, 4 DG, 13 LPC, 23 PC, 3 PE, 3 SM, and 5 TG were fully identified, illustrated 
in Figure 3.4. From gradient program C, 4 Cer_NS, 3 DG, 12 LPC, 18 PC, 3 PE, 4 SM, and 11 TG were fully identified, illustrated in Figure 3.5.

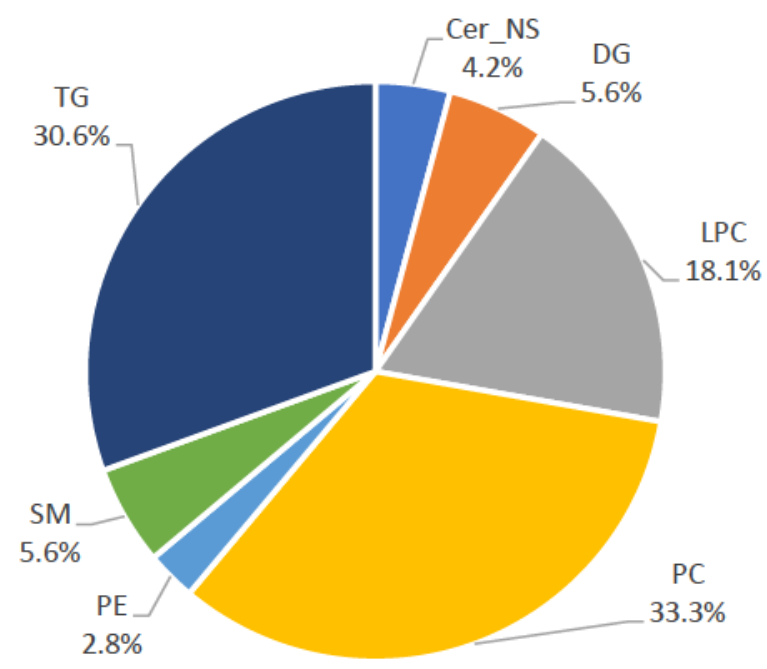

Figure 3.3: Relative distribution of lipids found in bovine liver using gradient program A

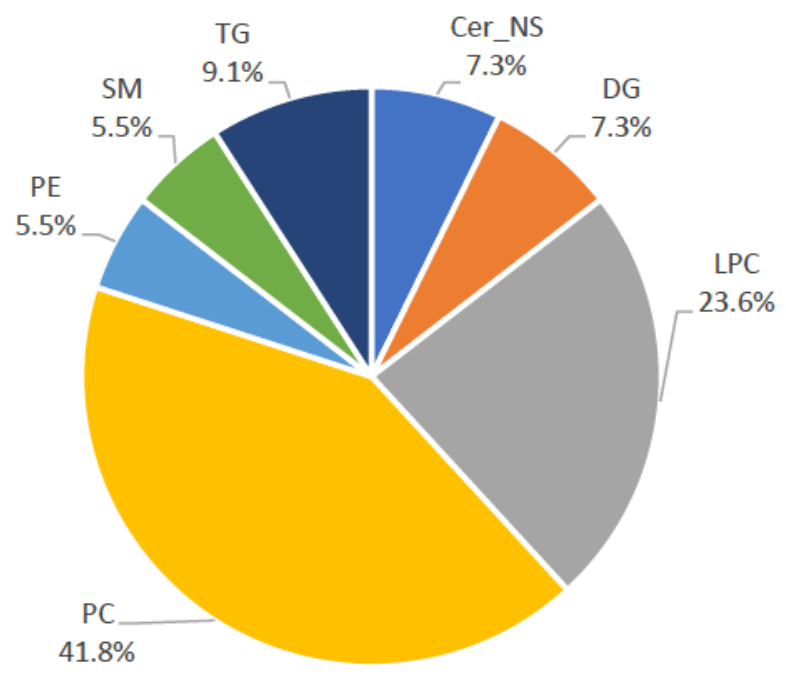

Figure 3.4: Relative distribution of lipids found in bovine liver using gradient program B 


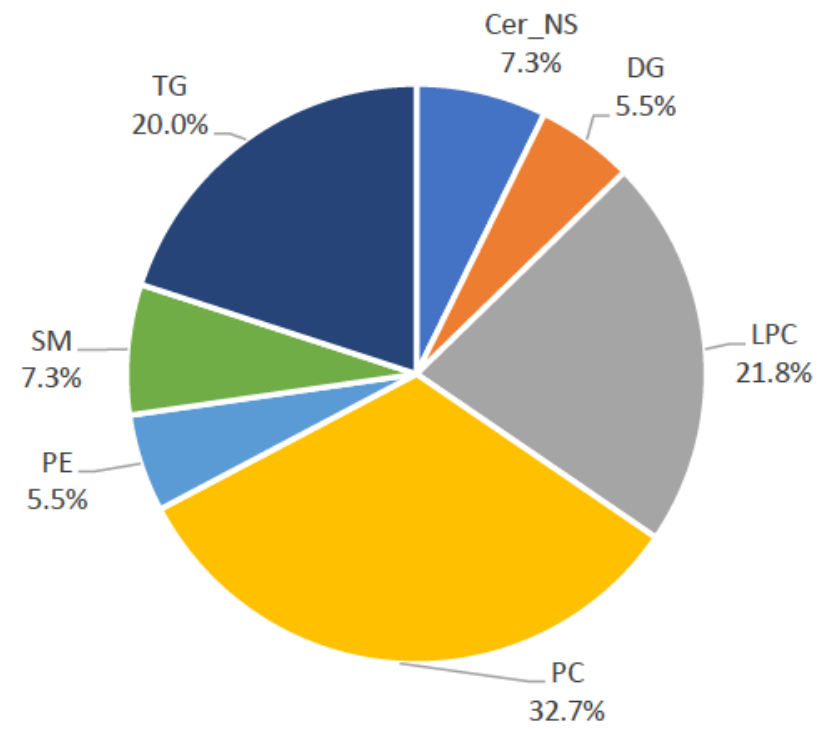

Figure 3.5: Relative distribution of lipids found in bovine liver using gradient program $C$ Lipids that were fully identified can be further illustrated in Figures 3.6 to 3.8 which demonstrates the overall distribution of each lipid species in a $\mathrm{m} / \mathrm{z}$ vs. time plot. By visual comparison, program A (Figure 3.6) illustrates higher degree of separation between lipid species within the same lipid class compared to the other two gradient programs tested. Examples include the Cer_NS, LPC and PC classes. The retention difference between Cer_NS d18:1_23:0 and Cer_NS d18:1_24:0 is $\Delta=0.959$ mins in gradient A, $\Delta=0.43$ mins in gradient $\mathrm{B}$ and $\Delta=0.424$ mins in gradient $\mathrm{C}$. The retention difference between LPC $0: 0 / 18: 2$ and LPC $18: 2 / 0: 0$ is $\Delta=0.582$ mins in gradient $\mathrm{A}, \Delta=0.148$ mins in gradient $\mathrm{B}$ and $\Delta=0.150$ mins in gradient C. The retention difference between PC 16:0_18:2 and PC $18: 0 \_18: 1$ is $\Delta=3.054$ mins in gradient $\mathrm{A}, \Delta=2.598$ mins in gradient $\mathrm{B}$ and $\Delta=2.569$ mins in gradient $\mathrm{C}$. This shows that a key advantage of using gradient program $\mathrm{A}$ is improved lipid species separation. 


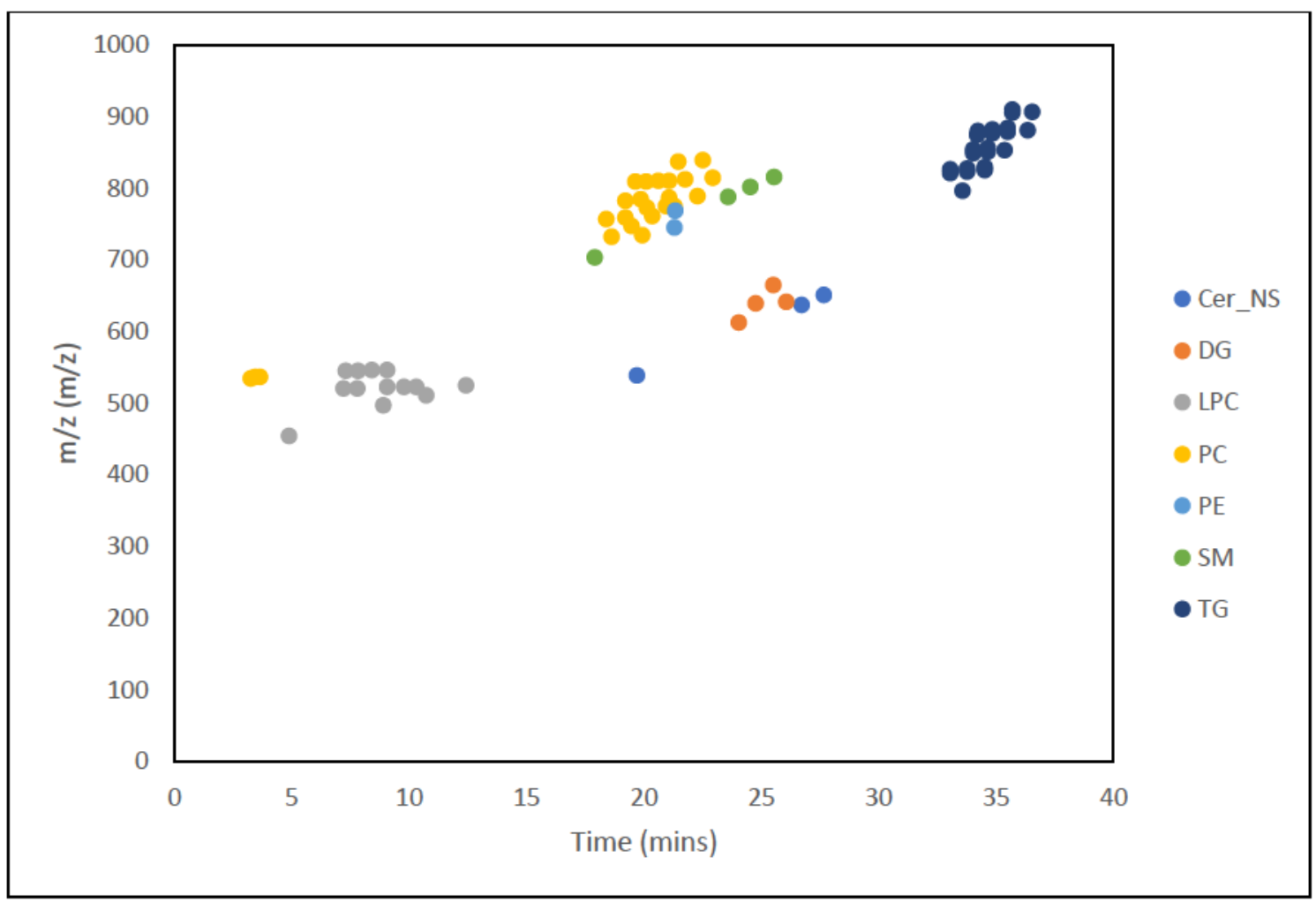

Figure 3.6: A m/z vs time (mins) plot of a 5-fold diluted bovine liver extract using gradient program $A$

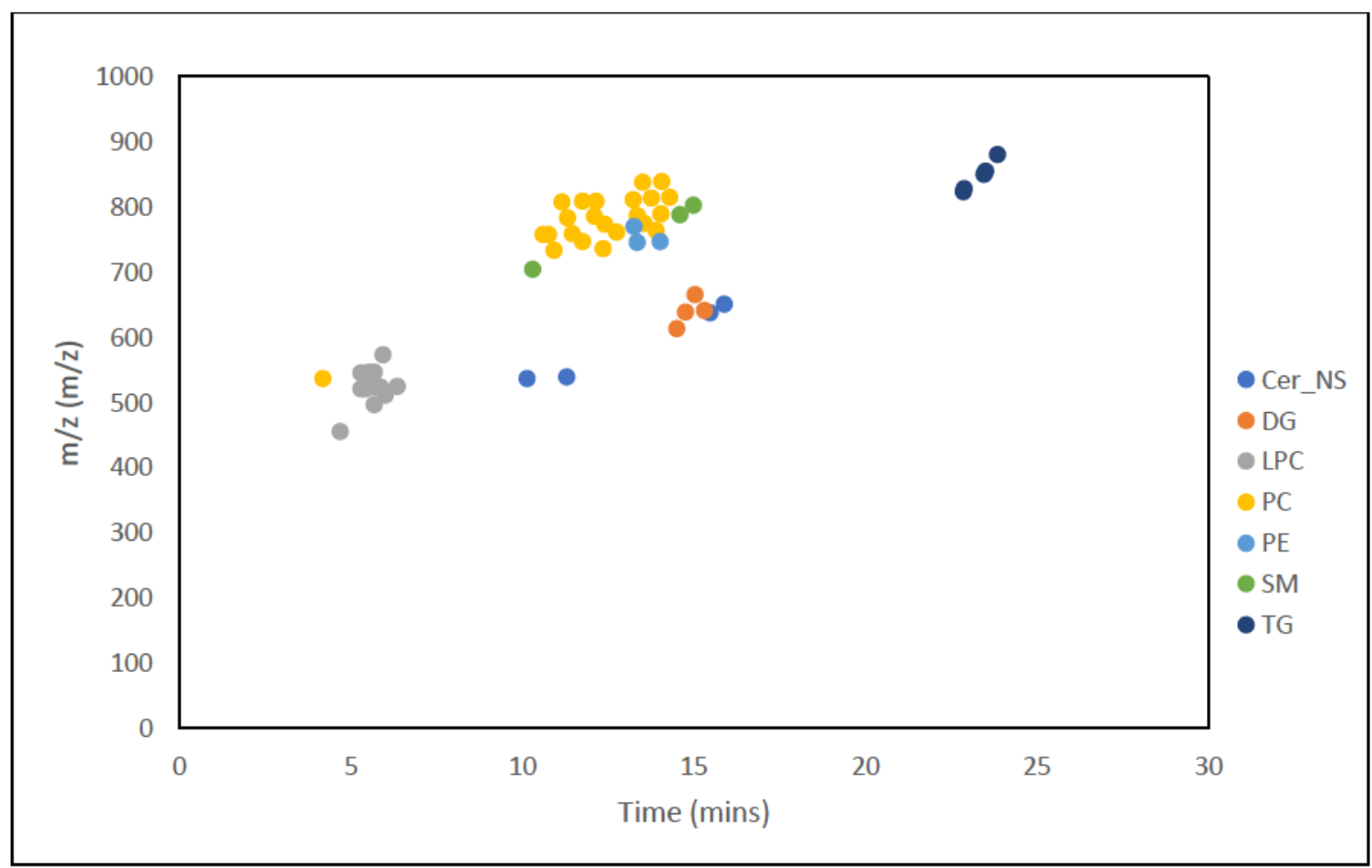

Figure 3.7: A m/z vs time (mins) plot of a 5-fold diluted bovine liver extract using gradient program $B$ 


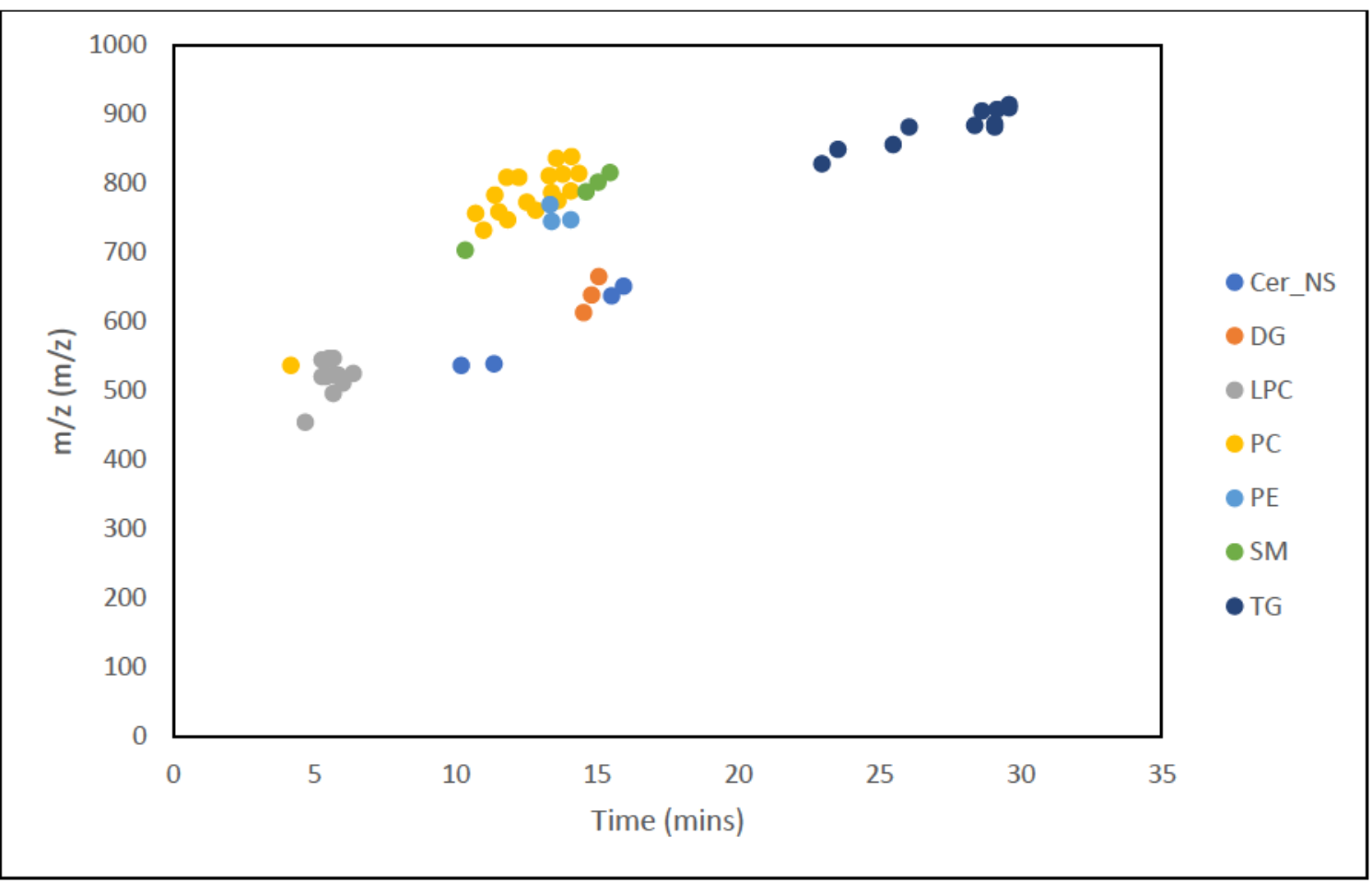

Figure 3.8: A m/z vs time (mins) plot of a 5-fold diluted bovine liver extract using gradient program $\mathrm{C}$

An overall summary is illustrated in Figure 3.9. Per lipid class, there are insignificant differences observed in the number of lipid species except for PC and TGs across all three gradients. As presented in Figure 3.9, Cer_NS, DG, LPC, PE, and SM all consist of a similar number of annotated lipids ( \pm 1 lipid species) via Agilent's Lipid Annotator when testing the three gradient programs. This slight deviation was considered insignificant as accurate annotation heavily relies on software and high-quality data since manual annotation in untargeted lipidomics studies is unrealistic. ${ }^{5}$ In the PC class, it was found that more lipid species were fully identified using gradient A compared to the other two gradients, but gradient B did not suffer a significant decrease. However, using gradient program C resulted in a $25 \%$ reduction of fully identified PCs. Upon investigation, there were no differences between the mobile phases at the elution times; PCs were eluting at $100 \%$ solvent $\mathrm{C}\left(1: 3 \mathrm{IPA}: \mathrm{MeOH}\right.$ with $\left.10 \mathrm{mM} \mathrm{NH}_{4} \mathrm{HCO}_{2}\right)$ in both programs $\mathrm{B}$ and 
C, nor were there changes to either the QTOF or Lipid Annotator's parameters. A possible explanation lies within the ability of Lipid Annotator's algorithm to annotate accurately. This can be supported based on the data acquired. Analysis of PCs (Table 3.1) acquired from all three gradient programs demonstrates the limitations of Lipid Annotator. For example, a PC with a $\mathrm{m} / \mathrm{z}=774.60$ Da may be annotated as both a sum composition and a dominant constituent if discern based on fragmentation patterns. This may increase or decrease the number of fully annotated lipids reported.

Table 3.1: PCs annotated from Lipid Annotator using gradient programs A, B and C.

\begin{tabular}{|c|c|c|c|c|}
\hline Retention Time (mins) & $\mathrm{m} / \mathrm{z}$ & Compound Name & Formula & Gradient \\
\hline 3.47 & 536.3344 & PC $18: 1$ & $\mathrm{C} 26 \mathrm{H} 50 \mathrm{NO} 8 \mathrm{P}$ & $\mathrm{A}$ \\
\hline 3.67 & 536.3344 & PC 18:1 & $\mathrm{C} 26 \mathrm{H} 50 \mathrm{NO} 8 \mathrm{P}$ & $A$ \\
\hline 4.185 & 536.3348 & PC 18:1 & $\mathrm{C} 26 \mathrm{H} 50 \mathrm{NO} 8 \mathrm{P}$ & B \\
\hline 4.173 & 536.3357 & PC 18:1 & $\mathrm{C} 26 \mathrm{H} 50 \mathrm{NO} 8 \mathrm{P}$ & $\mathrm{C}$ \\
\hline 18.652 & 732.5546 & PC 16:0_16:1 & $\mathrm{C} 40 \mathrm{H} 78 \mathrm{NO} 0 \mathrm{P}$ & $A$ \\
\hline 10.933 & 732.5540 & PC 16:0_16:1 & $\mathrm{C} 40 \mathrm{H} 78 \mathrm{NO} 8 \mathrm{P}$ & B \\
\hline 10.991 & 732.5548 & PC 16:0_16:1 & $\mathrm{C} 40 \mathrm{H} 78 \mathrm{NO} 8 \mathrm{P}$ & $\mathrm{C}$ \\
\hline 19.967 & 734.5698 & PC 16:0_16:0 & $\mathrm{C} 40 \mathrm{H} 80 \mathrm{NO} 8 \mathrm{P}$ & $A$ \\
\hline 12.36 & 734.5688 & PC 16:0_16:0 & $\mathrm{C} 40 \mathrm{H} 80 \mathrm{NO} 8 \mathrm{P}$ & B \\
\hline 19.479 & 746.5696 & PC 15:0_18:1 & C41H80NO8P & $A$ \\
\hline 11.762 & 746.5695 & PC 16:0_17:1 & C41H80NO8P & B \\
\hline 11.839 & 746.5706 & PC 16:0_17:1 & C41H80NO8P & $\mathrm{C}$ \\
\hline 18.41 & 756.5540 & PC 16:0_18:3 & $\mathrm{C} 42 \mathrm{H} 78 \mathrm{NO} 8 \mathrm{P}$ & $A$ \\
\hline 10.614 & 756.5539 & PC 16:0_18:3 & $\mathrm{C} 42 \mathrm{H} 78 \mathrm{NO} 8 \mathrm{P}$ & B \\
\hline 10.717 & 756.5550 & PC 16:0_18:3 & $\mathrm{C} 42 \mathrm{H} 78 \mathrm{NO} 8 \mathrm{P}$ & $\mathrm{C}$ \\
\hline 10.77 & 756.5531 & PC 18:1_16:2 & $\mathrm{C} 42 \mathrm{H} 78 \mathrm{NO} 8 \mathrm{P}$ & B \\
\hline 19.234 & 758.5715 & PC 16:0_18:2 & $\mathrm{C} 42 \mathrm{H} 80 \mathrm{NO} 8 \mathrm{P}$ & $A$ \\
\hline 11.459 & 758.5709 & PC 16:0_18:2 & $\mathrm{C} 42 \mathrm{H} 80 \mathrm{NO} 8 \mathrm{P}$ & B \\
\hline 11.525 & 758.5721 & PC 16:0_18:2 & C42H80NO8P & $\mathrm{C}$ \\
\hline 20.373 & 760.5870 & PC 16:0_18:1 & $\mathrm{C} 42 \mathrm{H} 82 \mathrm{NO} 8 \mathrm{P}$ & $A$ \\
\hline 12.751 & 760.5867 & PC 16:0_18:1 & $\mathrm{C} 42 \mathrm{H} 82 \mathrm{NO} 8 \mathrm{P}$ & B \\
\hline 12.845 & 760.5876 & PC 16:0_18:1 & $\mathrm{C} 42 \mathrm{H} 82 \mathrm{NO} 8 \mathrm{P}$ & $\mathrm{C}$ \\
\hline 13.914 & 762.6002 & PC 16:0_18:0 & $\mathrm{C} 42 \mathrm{H} 84 \mathrm{NO} 8 \mathrm{P}$ & B \\
\hline 20.155 & 772.5853 & PC 17:0_18:2 & $\mathrm{C} 43 \mathrm{H} 82 \mathrm{NO} 8 \mathrm{P}$ & A \\
\hline
\end{tabular}




\begin{tabular}{|c|c|c|c|c|}
\hline 12.421 & 772.5846 & PC 17:0_18:2 & $\mathrm{C} 43 \mathrm{H} 82 \mathrm{NO} 8 \mathrm{P}$ & $B$ \\
\hline 12.536 & 772.5859 & PC 17:0_18:2 & C43H82N08P & $\mathrm{C}$ \\
\hline 21.324 & 774.6013 & PC 17:0_18:1 & $\mathrm{C} 43 \mathrm{H} 84 \mathrm{NO} 8 \mathrm{P}$ & A \\
\hline 20.977 & 774.6008 & PC 35:1 & С43H84NO8P & $A$ \\
\hline 13.571 & 774.6006 & PC 35:1 & $\mathrm{C} 43 \mathrm{H} 84 \mathrm{NO} 8 \mathrm{P}$ & B \\
\hline 13.623 & 774.6019 & PC 18:0_17:1 & $\mathrm{C} 43 \mathrm{H} 84 \mathrm{NO} 8 \mathrm{P}$ & $\mathrm{C}$ \\
\hline 19.233 & 782.5697 & PC 16:0_20:4 & $\mathrm{C} 44 \mathrm{H} 80 \mathrm{NO} 8 \mathrm{P}$ & A \\
\hline 11.336 & 782.5699 & PC 16:0_20:4 & C44H80NO8P & $B$ \\
\hline 11.397 & 782.5709 & PC 16:0_20:4 & C44H80NO8P & $\mathrm{C}$ \\
\hline 19.888 & 784.5864 & PC 16:0_20:3 & $\mathrm{C} 44 \mathrm{H} 82 \mathrm{NO} 8 \mathrm{P}$ & A \\
\hline 12.105 & 784.5860 & PC 16:0_20:3 & $\mathrm{C} 44 \mathrm{H} 82 \mathrm{NO} 8 \mathrm{P}$ & B \\
\hline 21.081 & 786.6023 & PC 18:0_18:2 & $\mathrm{C} 44 \mathrm{H} 84 \mathrm{NO} 8 \mathrm{P}$ & A \\
\hline 13.353 & 786.6018 & PC 18:0_18:2 & $\mathrm{C} 44 \mathrm{H} 84 \mathrm{NO} 8 \mathrm{P}$ & B \\
\hline 13.417 & 786.6032 & PC 18:0_18:2 & C44H84NO8P & $\mathrm{C}$ \\
\hline 22.288 & 788.6181 & PC 18:0_18:1 & $\mathrm{C} 44 \mathrm{H} 86 \mathrm{NO} 8 \mathrm{P}$ & A \\
\hline 14.057 & 788.6173 & PC 18:0_18:1 & $\mathrm{C} 44 \mathrm{H} 86 \mathrm{NO} 8 \mathrm{P}$ & B \\
\hline 14.094 & 788.6193 & PC 18:0_18:1 & $\mathrm{C} 44 \mathrm{H} 86 \mathrm{NO} 8 \mathrm{P}$ & $\mathrm{C}$ \\
\hline 19.644 & 808.5848 & PC 38:5 & $\mathrm{C} 46 \mathrm{H} 82 \mathrm{NO} 8 \mathrm{P}$ & A \\
\hline 20.102 & 808.5837 & PC 38:5 & $\mathrm{C} 46 \mathrm{H} 82 \mathrm{NO} 08 \mathrm{P}$ & $A$ \\
\hline 11.757 & 808.5852 & PC 16:0_22:5 & $\mathrm{C} 46 \mathrm{H} 82 \mathrm{NO} 8 \mathrm{P}$ & B \\
\hline 12.151 & 808.5825 & PC 38:5 & $\mathrm{C} 46 \mathrm{H} 82 \mathrm{NO} 8 \mathrm{P}$ & B \\
\hline 11.815 & 808.5861 & PC 38:5 & $\mathrm{C} 46 \mathrm{H} 82 \mathrm{NO} 8 \mathrm{P}$ & $\mathrm{C}$ \\
\hline 12.252 & 808.5839 & PC 18:2_20:3 & $\mathrm{C} 46 \mathrm{H} 82 \mathrm{NO} 8 \mathrm{P}$ & C \\
\hline 20.637 & 810.6011 & PC 16:0_22:4 & $\mathrm{C} 46 \mathrm{H} 84 \mathrm{NO} 8 \mathrm{P}$ & A \\
\hline 21.088 & 810.6016 & PC 18:0_20:4 & C46H84NO8P & A \\
\hline 13.248 & 810.6016 & PC 18:0_20:4 & $\mathrm{C} 46 \mathrm{H} 84 \mathrm{NO} 8 \mathrm{P}$ & B \\
\hline 13.319 & 810.6026 & PC 18:0_20:4 & $\mathrm{C} 46 \mathrm{H} 84 \mathrm{NO} 8 \mathrm{P}$ & $\mathrm{C}$ \\
\hline 21.78 & 812.6181 & PC 18:0_20:3 & $\mathrm{C} 46 \mathrm{H} 86 \mathrm{NO} 8 \mathrm{P}$ & $A$ \\
\hline 13.77 & 812.6171 & PC 18:0_20:3 & $\mathrm{C} 46 \mathrm{H} 86 \mathrm{NO} 8 \mathrm{P}$ & B \\
\hline 13.814 & 812.619 & PC 18:0_20:3 & C46H86NO8P & $\mathrm{C}$ \\
\hline 22.538 & 838.6332 & PC 18:0_22:4 & C48H88NO8P & $A$ \\
\hline 14.085 & 838.634 & PC 18:0_22:4 & C48H88NO8P & B \\
\hline 14.119 & 838.6346 & PC 18:0_22:4 & C48H88NO8P & $\mathrm{C}$ \\
\hline
\end{tabular}

With TGs, it was observed that more species were fully identified using gradient $A$ than gradient $\mathrm{B}$ or $\mathrm{C}$. Upon reviewing literature, it was found that a non-polar characteristic component must be optimized to disrupt any hydrophobic absorption of the lipids to the 
stationary phase and eliminate carryover. ${ }^{6}$ In an Agilent application note, the separation of TGs using $\mathrm{MeOH}$ containing gradients has consistently shown an overall lower resolution. ${ }^{7}$ By creating a gradient program that has a higher percentage of IPA instead of $\mathrm{MeOH}$ (less polar mobile phase overall), this allows more neutral lipids like TG to be annotated. This can be supported as the lipid gradient switched from 1:3 IPA:MeOH with $10 \mathrm{mM} \mathrm{NH}_{4} \mathrm{HCO}_{2}$ to $3: 1$ IPA:MeOH with $10 \mathrm{mM} \mathrm{NH}_{4} \mathrm{HCO}_{2}$ in gradient $\mathrm{C}$ at the $25^{\text {th }}$ minute mark allowed for 8 more TGs to be identified compared to B.

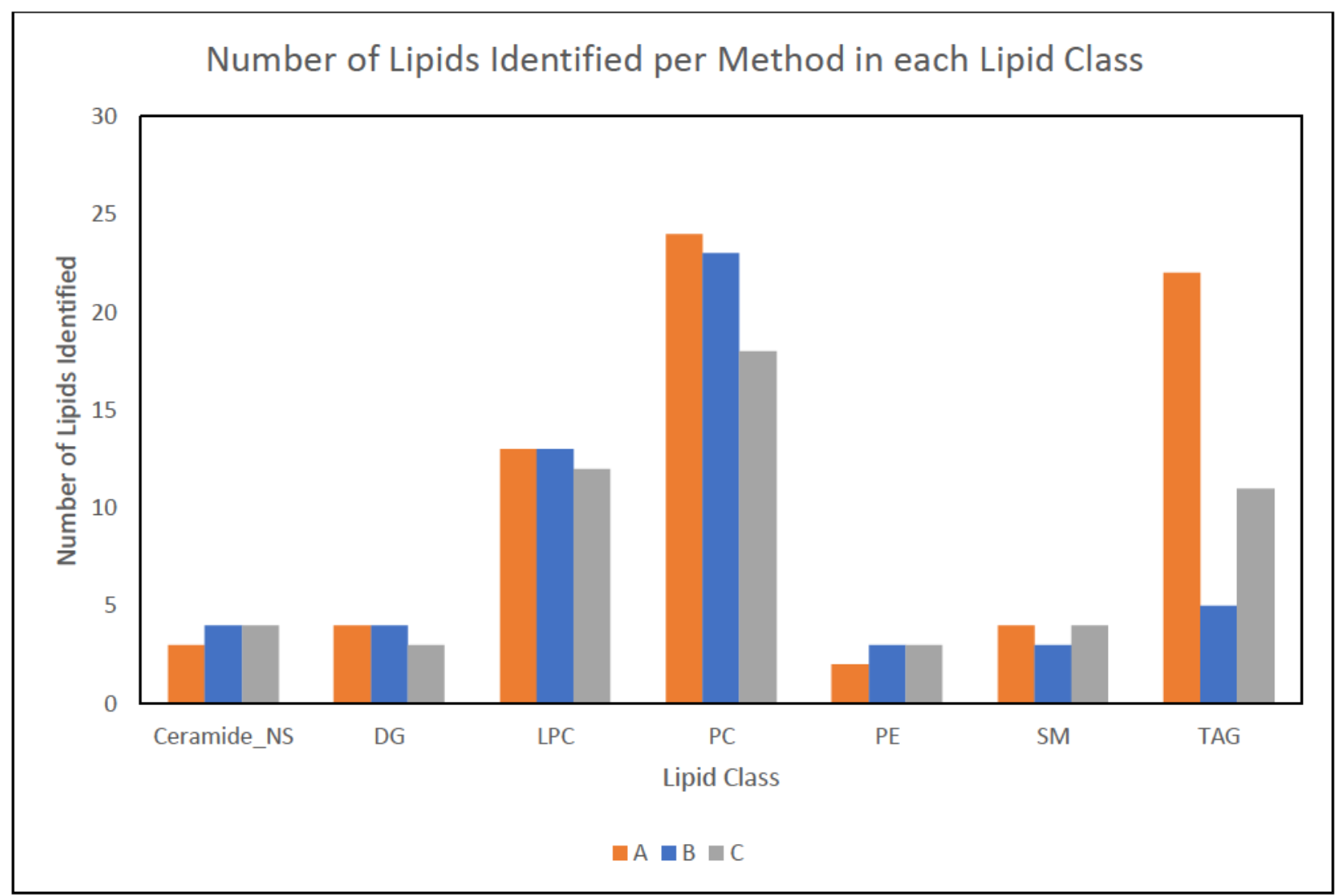

Figure 3.9: Number of lipids identified in each lipid class using gradients $A, B$ and $C$ from bovine liver.

To ensure accurate analysis, the internal standard LPC C13:0 was also analyzed in MassHunter Qualitative, Figure 3.10. The LPC of interest has an exact mass of 453.29 Da. Therefore, the protonated mass, $[\mathrm{M}+\mathrm{H}]^{+}$, should be $454.29 \mathrm{Da}$. In MS/MS acquisition 
mode, an EIC of 454.29 Da was performed in all three gradient programs, along the corresponding +ESI scans. Analysis of the lipid standard was acquired in MS/MS acquisition with positive ion mode (Figures 3.11 to 3.13 ). By acquiring in MS/MS mode, an observable distinct 184.07 Da fragment, representing the polar head group of LPC C13:0, could be observed. From these results, the lipid was determined to be observable in all three gradient programs using the 6546 QTOF.

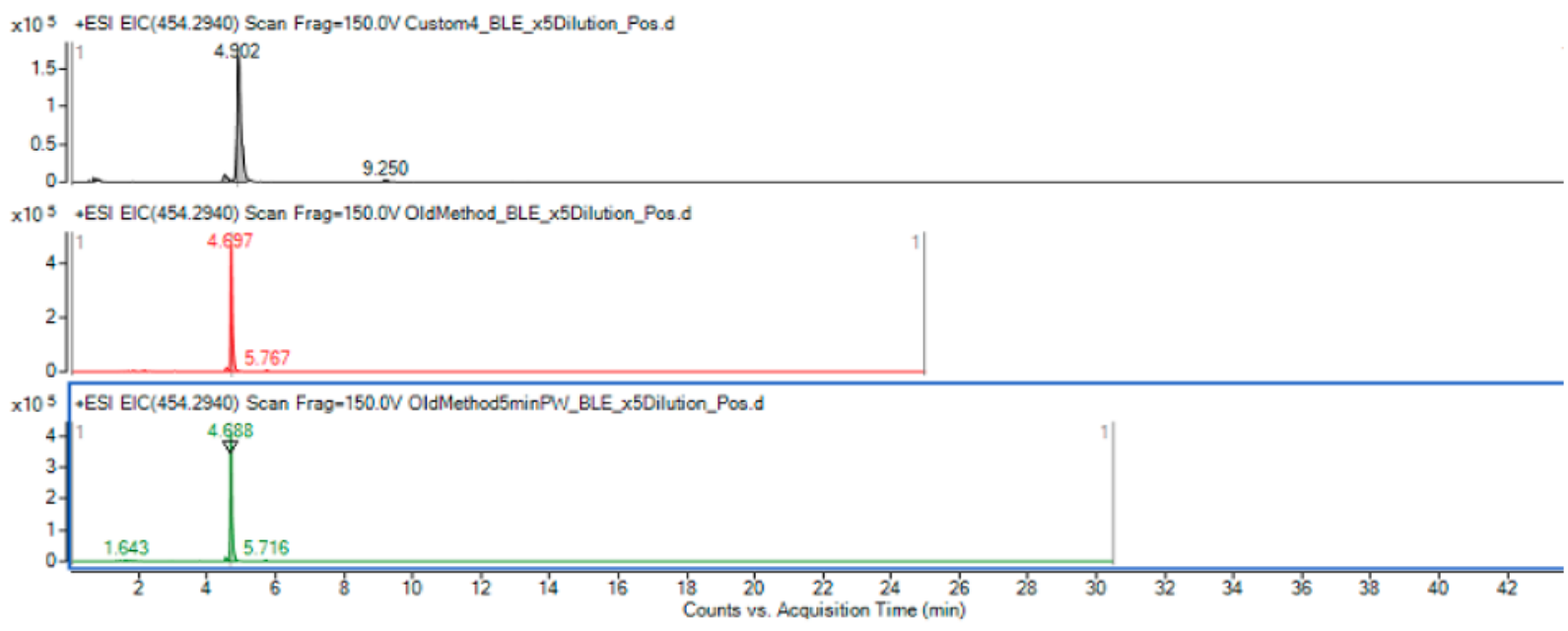

Figure 3.10: EIC of the internal standard LPC C13:0 in all three gradient programs from a bovine liver extract. Grey: Gradient A, Red: Gradient B, Green: Gradient C.

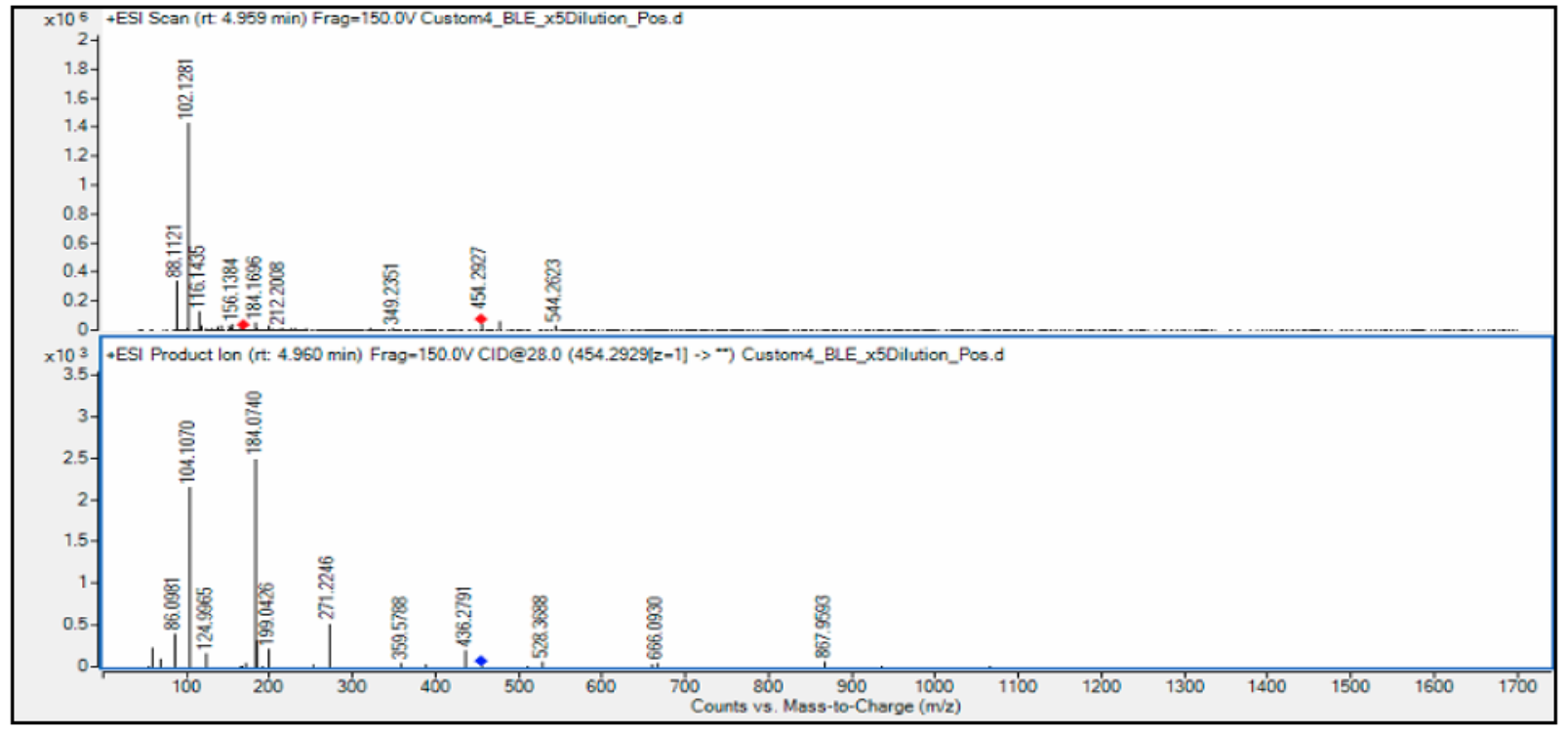

Figure 3.11: ESI+ MS and MS/MS spectra of LPC C13:0 (m/z 454.29) using gradient program A. Blue diamond represents the parent ion. 


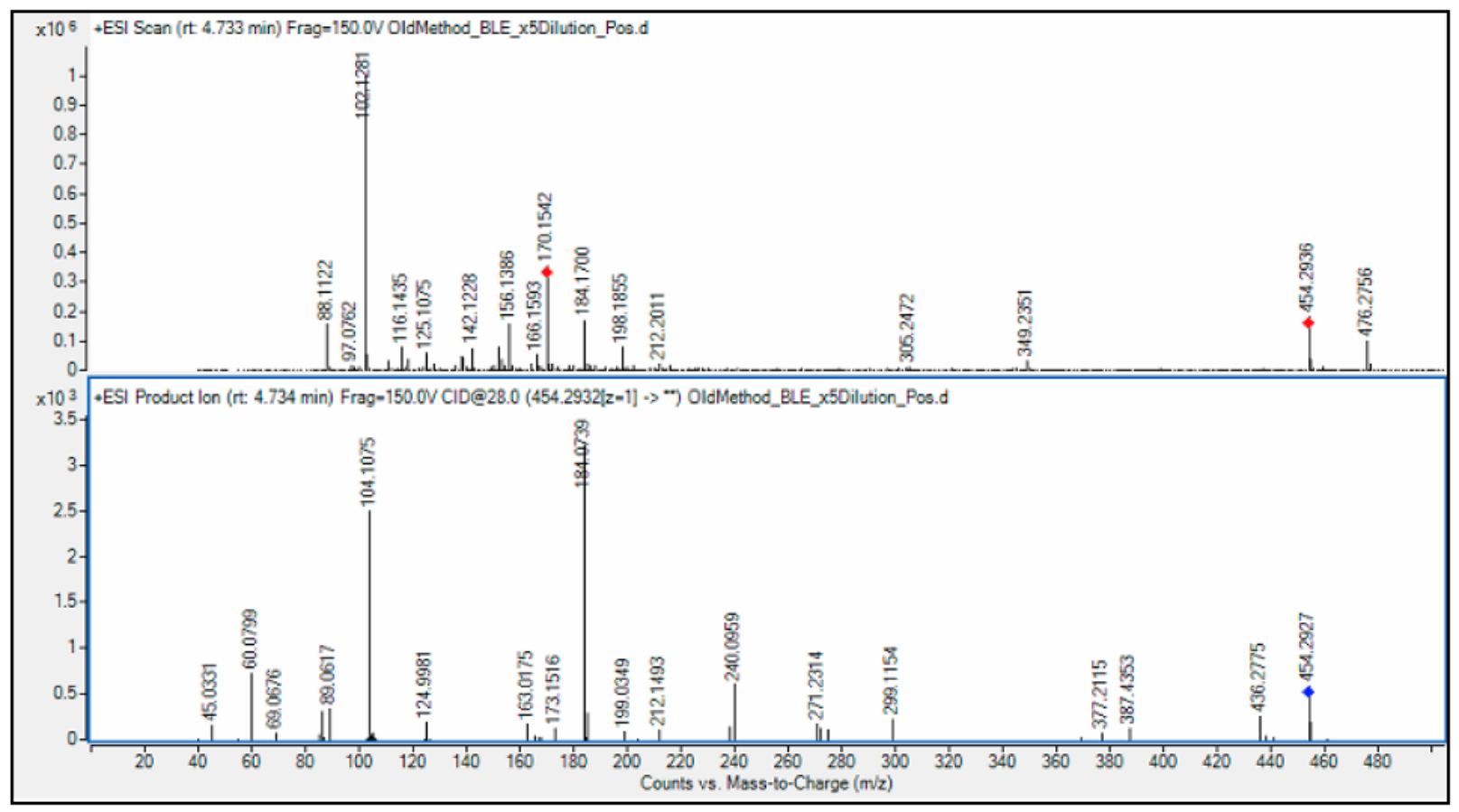

Figure 3.12: ESI+ MS and MS/MS spectra of LPC C13:0 (m/z 454.29) using gradient program B. Blue diamond represents the parent ion.

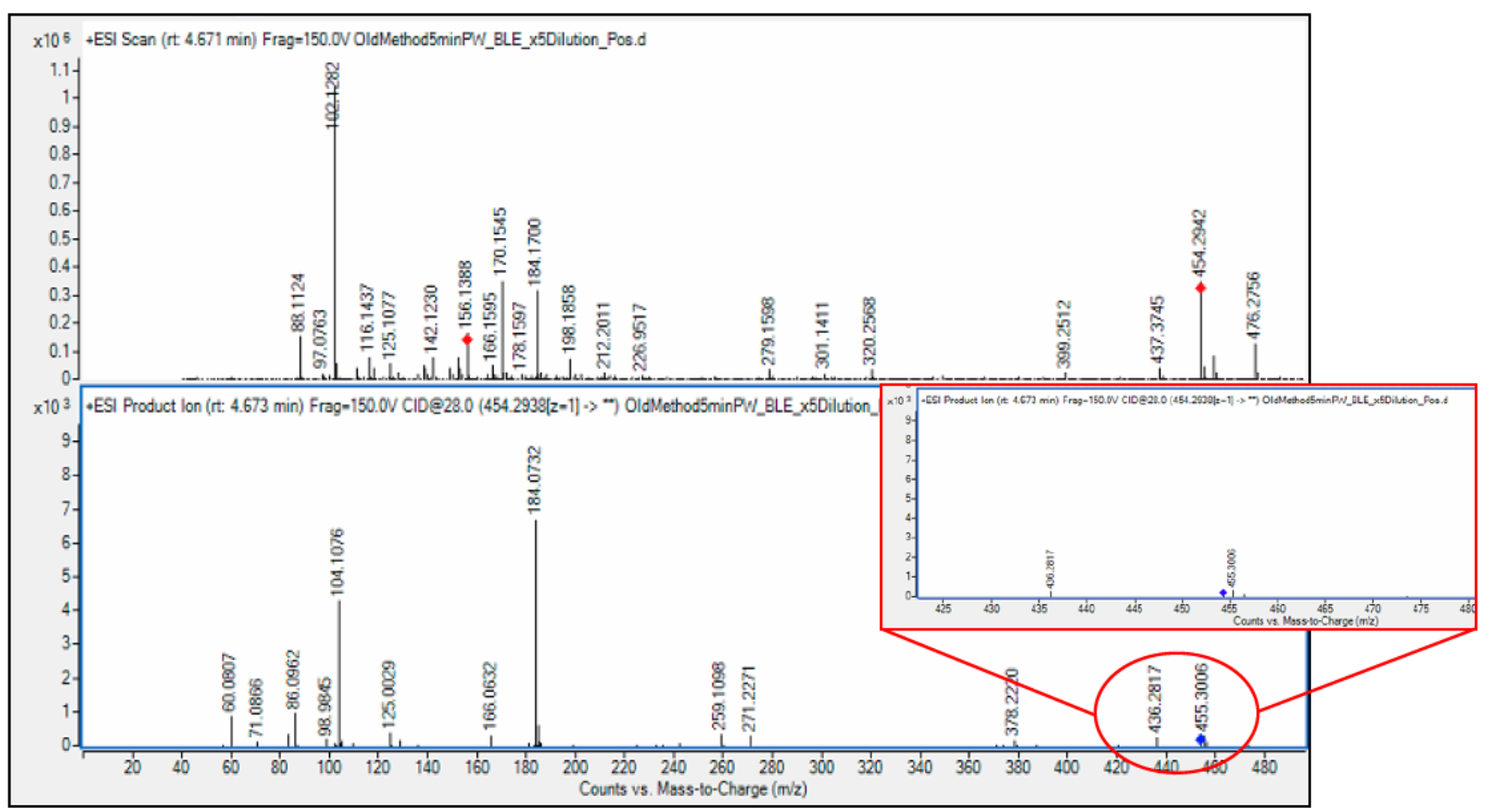

Figure 3.13: ESI+ MS and MS/MS spectra of LPC C13:0 (m/z 454.29) using gradient program $\mathrm{C}$. Blue diamond represents the parent ion. 
The next objective of this research is to verify the results observed with rat liver tissues. In the same manner, lipid extraction was performed as per Chapter 3.2. Once extracted, lipids were analyzed using three different gradient programs described in Chapter 3.2. Analysis of the MS data acquired from the rat liver tissues yielded a total of 47 lipids, 28 lipids and, 34 lipids from gradient programs A, B, and C respectively using Agilent's Lipid Annotator. From gradient program A, 0 DG, 7 LPC, 15 PC, 0 PE, 1 SM, and 24 TG were fully identified. From gradient program B, 2 DG, 5 LPC, 16 PC, 0 PE, 1 $\mathrm{SM}$, and $4 \mathrm{TG}$ were fully identified. From gradient program C, $2 \mathrm{DG}, 7 \mathrm{LPC}, 14 \mathrm{PC}, 1 \mathrm{PE}$, $0 \mathrm{SM}$, and $10 \mathrm{TG}$ were fully identified.

Figures 3.14 to 3.16 describe the distribution of every annotated lipid species in a $\mathrm{m} / \mathrm{z}$ vs. time plot. By comparison, program A (Figure 3.14) demonstrated a higher degree of separation in the LPC class compared to the other two gradient programs (Figures 3.15 and 3.16). For example, the retention difference between LPC 0:0/18:2 and LPC 0:0/20:4 is $\Delta=0.079$ mins in gradient A, $\Delta=0.00$ mins in gradient $\mathrm{B}$ and $\Delta=0.00$ mins in gradient $\mathrm{C}$. However, this was not observed in other lipid classes. Unlike the previous experiment, PCs did not exhibit the same separation observed in gradient program A. Despite the inconsistent results, more TGs were annotated using gradient $\mathrm{A}$ compared to $\mathrm{B}$ or $\mathrm{C}$. 


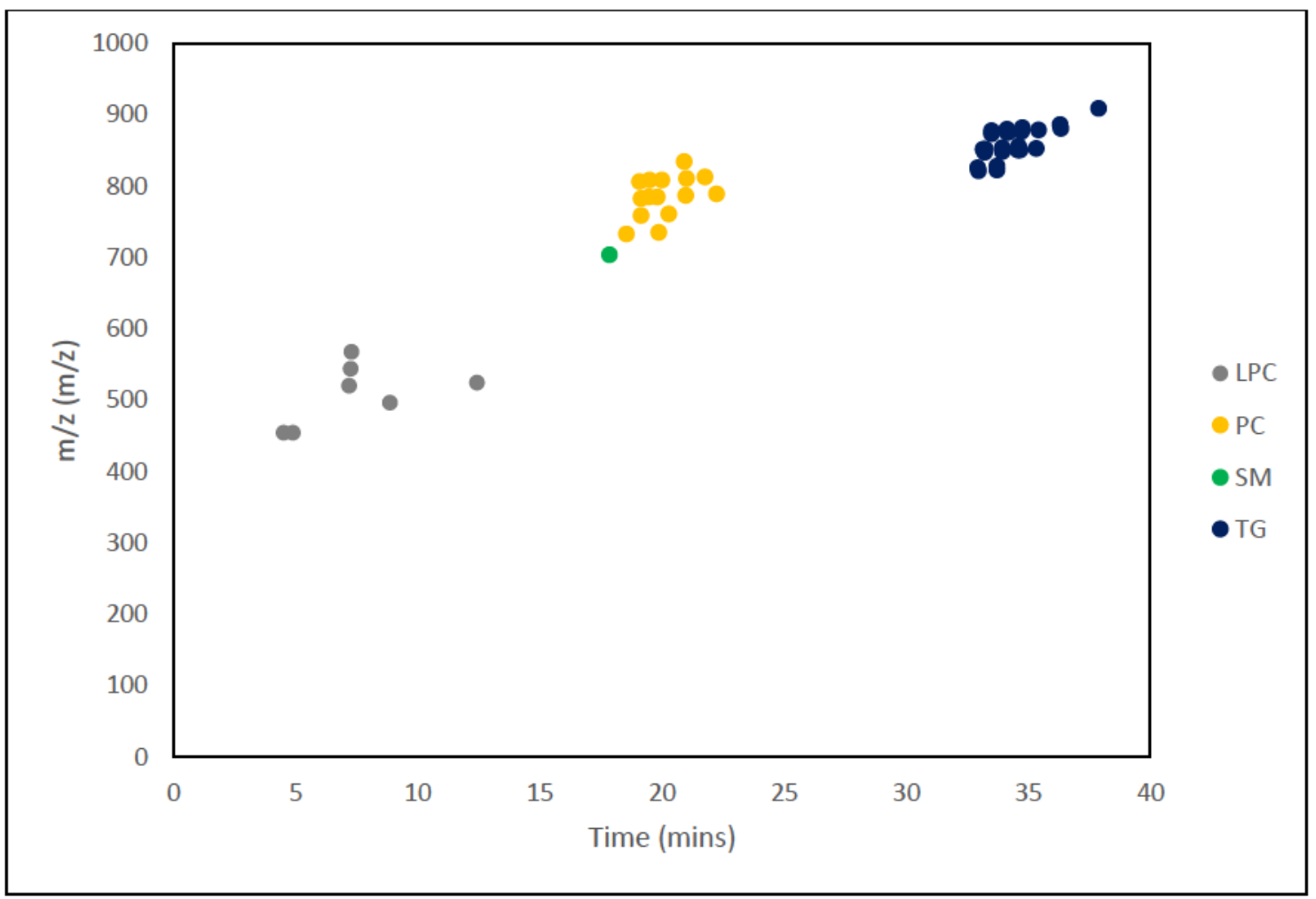

Figure 3.14: A m/z vs time (mins) plot of a 2 -fold diluted rat liver extract using gradient program $A$

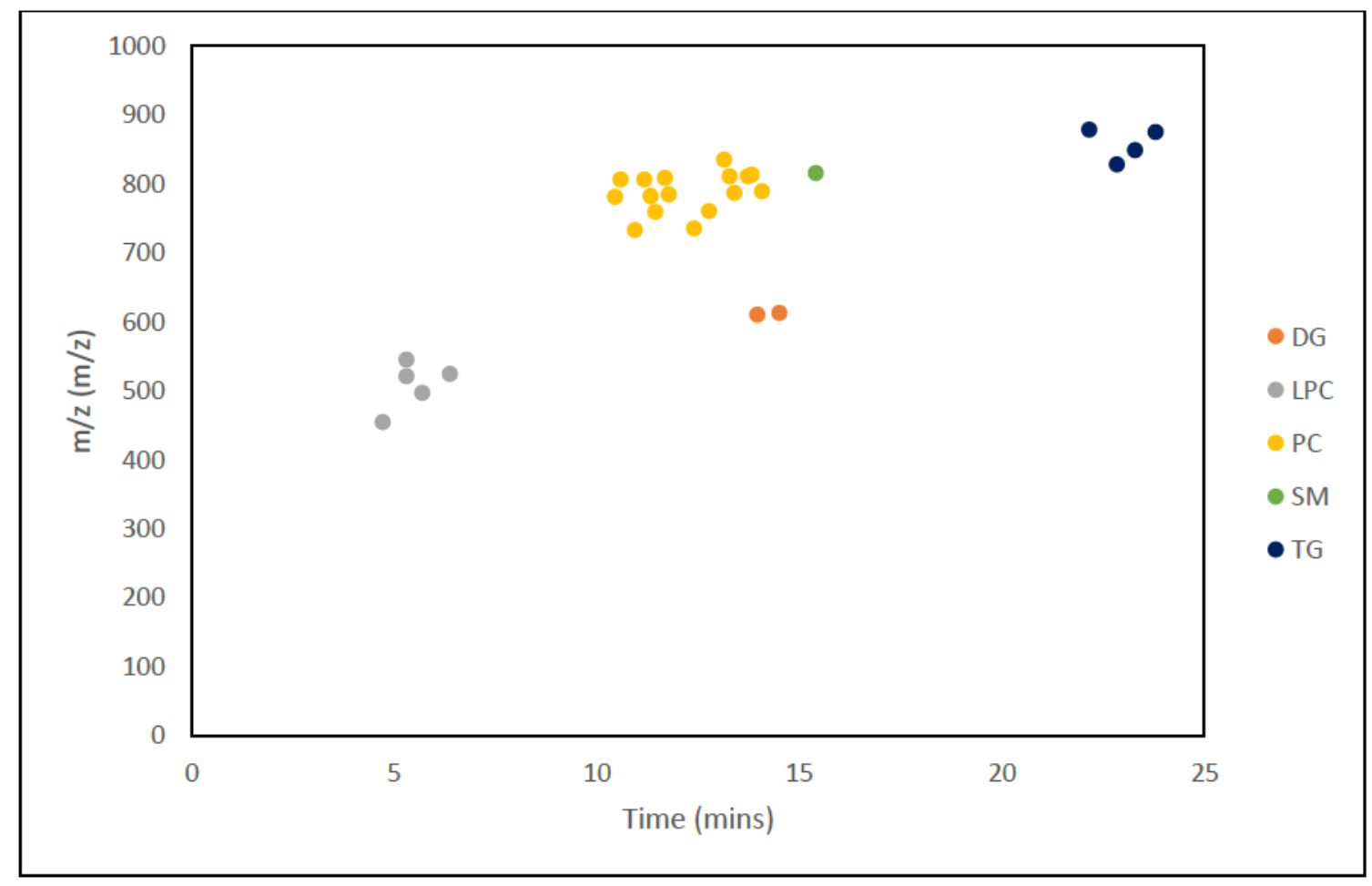

Figure 3.15: A m/z vs time (mins) plot of a 2-fold diluted rat liver extract using gradient program B 


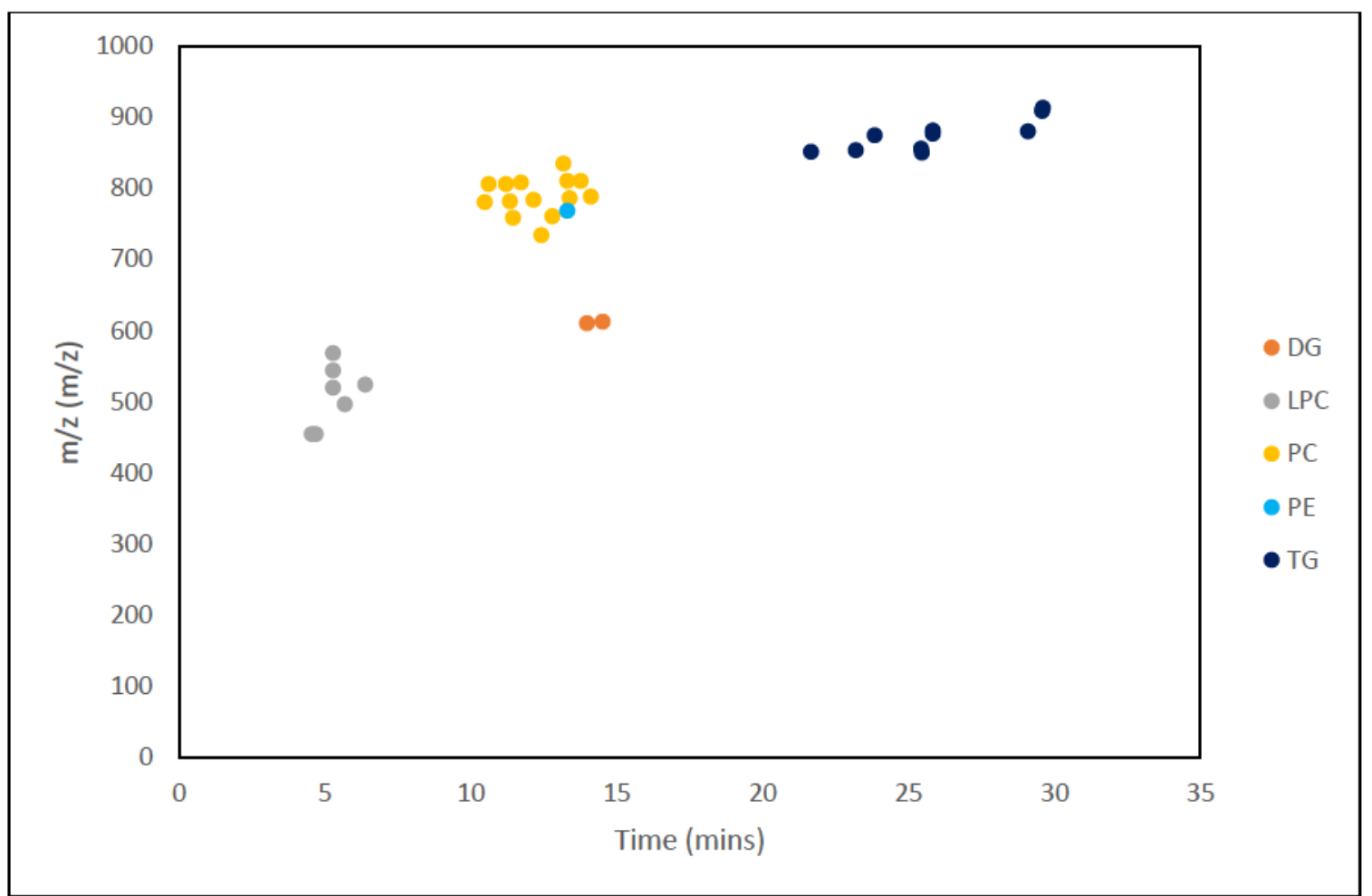

Figure 3.16: A m/z vs time (mins) plot of a 2 -fold diluted rat liver extract using gradient program $\mathrm{C}$

An overall summary is illustrated in Figure 3.17. Per lipid class, there are insignificant differences observed in the number of lipid species except for TGs across all three gradients. Like the previous experiment, DG, LPC, PE and SM all consist of similar number of annotated lipids ( \pm 2 lipid species) via Agilent's Lipid Annotator when testing the three gradient programs. As observed, similar amounts of PCs were identified across all gradients not found in the previous experiment. With TGs, a similar pattern was observed between the bovine and rat livers. With gradient A, 24 TGs were annotated compared to 4 and 10 TGs using gradient $B$ and $C$, respectively. It was concluded that more TGs were fully identified using gradient $A$ than gradient $B$ or $C$ because of a less polar mobile phase. Upon literature review, it was found that many studies primarily used IPA (mixed with other solvents but a high $\%$ of IPA) as part of their solvent system. ${ }^{2}$ 


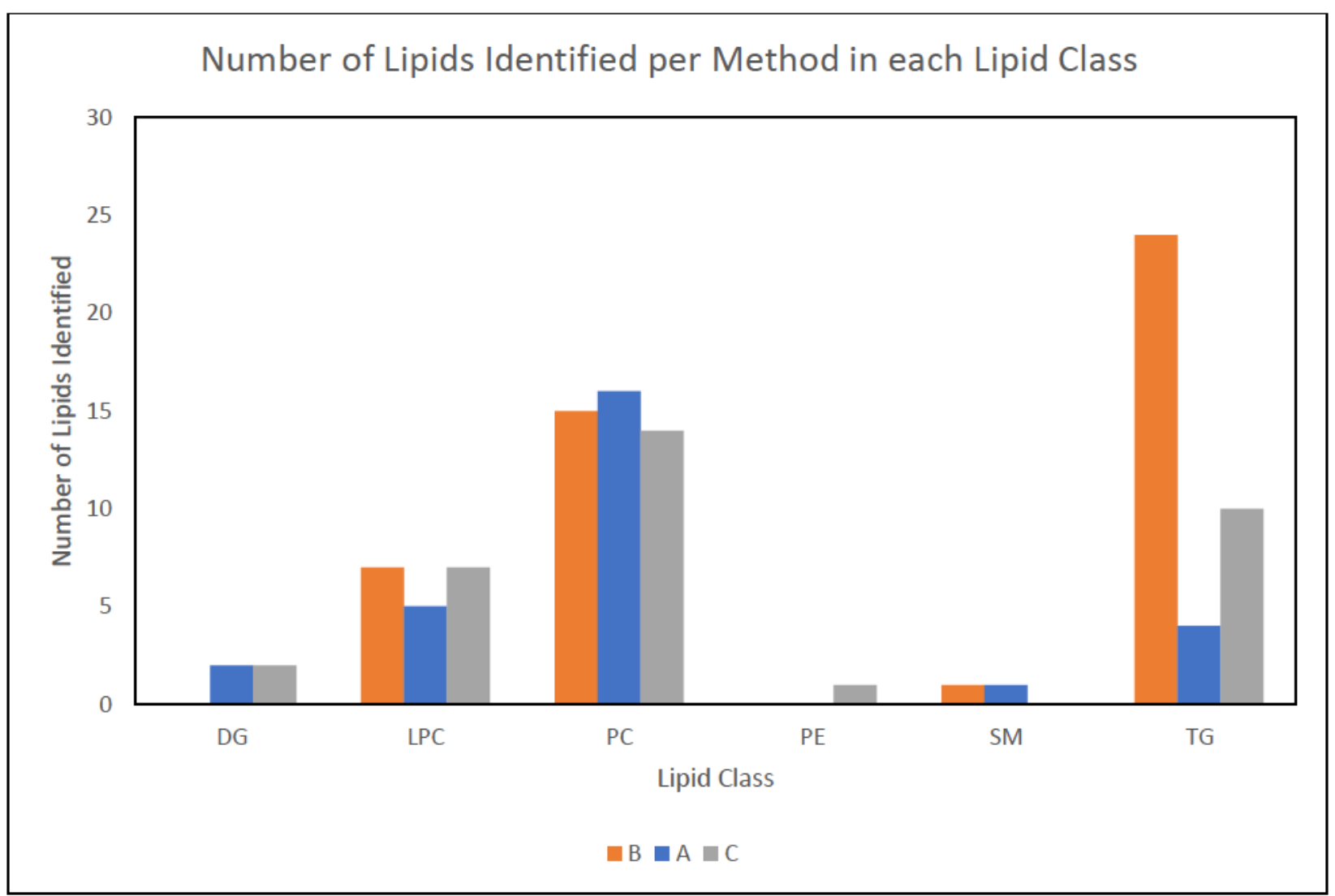

Figure 3.17: Number of lipids identified in each lipid class using gradients $A, B$ and $C$ from rat liver

The best method was determined to be gradient program A due to its ability to identify more neutral lipids (TGs) compared to the other two gradient programs. Despite the inconsistency of providing better separation in many lipid classes extracted from rat liver tissues, the benefit of providing better lipidomic coverage outweighs the negatives. Although this method may not be the foremost lipidomic gradient, due to a lack of time it was accepted as the method of choice. Additionally, it was determined that the last lipid identified elutes at 37.9 mins. With the rest of the gradient running at 3:1 IPA:MeOH with $10 \mathrm{mM}$ ammonium formate until 44 mins, it was created to eliminate potential carryover. Blanks were run subsequently to observe any carry over. The acquired data was imported 
into Lipid Annotator and no lipids were identified. This indicated that the method was suited to use sequentially without fear of significant carryover.

Sample dilution is critical to accurately determine the relative quantitation of each analyte of interest. Samples that are too dilute often result in issues with the limit of detection (LOD) or limit of quantification (LOQ). This negatively impacts the accuracy of analytes investigated which in this case, low abundance lipid species may not be detected/identified since it would be "buried" in noise. In contrast, samples that are highly concentrated result in detector saturation, which results in poor quantitation since it is beyond the instrument's linear dynamic range (LDR).

Originally, bovine lipid extracts were diluted 5-fold with $\mathrm{MeOH}$ where the solvent composition was $80: 20 \mathrm{MeOH}: \mathrm{EtOH}$. This dilution works well for bovine lipid extracts where it was above LOQ but within the LDR of the 6546 QTOF (previously tested by a former Ph.D. student). In the case of the rat lipid extract, a 5-fold dilution resulted in low signal intensities in many lipid classes and less identification. A 2-fold dilution was therefore tested and results improved. A consequence with a 2-fold dilution however was that the solvent composition of the sample was different since a $50: 50 \mathrm{MeOH}: \mathrm{EtOH}$ solution was created. To test whether solvent composition would affect retention time and chromatography, two bovine lipid extract solutions were made, both 5 -fold dilution, however, one consisted of a 80:20 MeOH:EtOH (20 $\mu \mathrm{L}$ sample in $\mathrm{EtOH}+80 \mu \mathrm{L} \mathrm{MeOH})$ while the other consisted of a $50: 50 \mathrm{MeOH}: \mathrm{EtOH}$ solution $(20 \mu \mathrm{L}$ sample in $\mathrm{EtOH}+30 \mu \mathrm{L}$ $\mathrm{EtOH}+50 \mathrm{MeOH}$ ). A table (Table 3.2) was generated to summarize lipid species identified and the retention difference between the two solutions. Alternatively, evapouration and resuspension in $\mathrm{MeOH}$ was considered, however, sample loss was of greater concern. 
Table 3.2: Retention time of lipid species extracted from bovine liver diluted 5-fold in $50 \%$ $\mathrm{MeOH}, 50 \% \mathrm{EtOH}$ and in $80 \% \mathrm{MeOH}, 20 \% \mathrm{EtOH}$.

\begin{tabular}{|c|c|c|c|c|}
\hline Lipid Species & $\begin{array}{c}\text { 1:1 MeOH:EtOH } \\
\text { RT(mins) }\end{array}$ & $\begin{array}{c}\text { 4:1 MeOH:EtOH } \\
\text { RT(mins) }\end{array}$ & $\Delta$ (mins) & $\Delta$ (secs) \\
\hline Cer_NS d18:1_16:0 & 19.558 & 19.592 & 0.034 & 2.04 \\
\hline Cer_NS d18:1_23:0 & 26.514 & 26.551 & 0.037 & 2.22 \\
\hline Cer_NS d18:1_24:0 & 27.501 & 27.525 & 0.024 & 1.44 \\
\hline DG 16:0_18:1 & 23.832 & 23.871 & 0.039 & 2.34 \\
\hline LPC 18:0/0:0 & 12.391 & 12.387 & 0.004 & 0.24 \\
\hline LPC 0:0/18:2 & 7.145 & 7.162 & 0.017 & 1.02 \\
\hline LPC 18:2/0:0 & 7.728 & 7.74 & 0.012 & 0.72 \\
\hline LPC 16:0/0:0 & 8.843 & 8.855 & 0.012 & 0.72 \\
\hline LPC 13:0/0:0 & 4.88 & 4.876 & 0.004 & 0.24 \\
\hline LPC 0:0/18:1 & 8.996 & 9.008 & 0.012 & 0.72 \\
\hline LPC 18:1/0:0 & 10.227 & 10.234 & 0.007 & 0.42 \\
\hline LPC 0:0/20:3 & 8.347 & 8.362 & 0.015 & 0.9 \\
\hline LPC 20:3/0:0 & 8.99 & 9.005 & 0.015 & 0.9 \\
\hline LPC 0:0/20:4 & 7.225 & 7.241 & 0.016 & 0.96 \\
\hline LPC 20:4/0:0 & 7.757 & 7.77 & 0.013 & 0.78 \\
\hline LPC 17:0/0:0 & 10.628 & 10.625 & 0.003 & 0.18 \\
\hline PC 2:0_16:2 & 3.239 & 3.25 & 0.011 & 0.66 \\
\hline PC 18:1 & 3.654 & 3.655 & 0.001 & 0.06 \\
\hline PC 16:0_16:1 & 18.529 & 18.552 & 0.023 & 1.38 \\
\hline PC 16:0_16:0 & 19.82 & 19.847 & 0.027 & 1.62 \\
\hline PC 16:0_17:1 & 19.349 & 19.349 & 0 & 0 \\
\hline PC 16:1_18:2 & 18.295 & 18.268 & 0.027 & 1.62 \\
\hline PC 16:0_18:2 & 19.097 & 19.12 & 0.023 & 1.38 \\
\hline PC 16:0_18:1 & 20.224 & 20.249 & 0.025 & 1.5 \\
\hline PC 17:0_18:2 & 19.999 & 20.047 & 0.048 & 2.88 \\
\hline PC 17:0_18:1 & 21.151 & 21.191 & 0.04 & 2.4 \\
\hline PC 16:0_20:4 & 19.09 & 19.113 & 0.023 & 1.38 \\
\hline PC 16:0_20:3 & 19.746 & 19.772 & 0.026 & 1.56 \\
\hline PC 16:0_22:5 & 19.936 & 19.963 & 0.027 & 1.62 \\
\hline PC 18:1_20:3 & 20.481 & 20.505 & 0.024 & 1.44 \\
\hline PC 18:0_20:4 & 20.906 & 20.929 & 0.023 & 1.38 \\
\hline PC 18:0_20:3 & 21.608 & 21.647 & 0.039 & 2.34 \\
\hline PC 18:0_22:6 & 20.801 & 20.836 & 0.035 & 2.1 \\
\hline PE 18:0_20:4 & 21.149 & 21.191 & 0.042 & 2.52 \\
\hline PE 18:0_18:2 & 21.133 & 21.173 & 0.04 & 2.4 \\
\hline SM d34:1 & 17.795 & 17.824 & 0.029 & 1.74 \\
\hline
\end{tabular}




\begin{tabular}{ccccc}
\hline SM d42:1 & 25.347 & 25.406 & 0.059 & 3.54 \\
SM d40:1 & 23.397 & 23.45 & 0.053 & 3.18 \\
TG 14:0_16:0_16:0 & 33.482 & 33.502 & 0.02 & 1.2 \\
TG 14:0_16:0_18:2 & 32.973 & 32.983 & 0.01 & 0.6 \\
TG 14:0_16:0_18:1 & 33.661 & 33.679 & 0.018 & 1.08 \\
TG 16:0_16:0_16:0 & 34.4 & 34.435 & 0.035 & 2.1 \\
TG 48:0 & 34.398 & 34.431 & 0.033 & 1.98 \\
TG 16:0_16:0_18:2 & 33.924 & 33.956 & 0.032 & 1.92 \\
TG 16:0_16:0_18:1 & 34.543 & 34.576 & 0.033 & 1.98 \\
TG 16:0_16:0_18:0 & 35.264 & 35.302 & 0.038 & 2.28 \\
TG 16:0_18:1_18:2 & 34.105 & 34.14 & 0.035 & 2.1 \\
TG 16:0_18:1_18:1 & 34.738 & 34.77 & 0.032 & 1.92 \\
TG 16:0_18:0_18:0 & 36.269 & 36.311 & 0.042 & 2.52 \\
TG 16:0_18:0_18:2 & 34.755 & 34.781 & 0.026 & 1.56 \\
TG 52:1 & 35.413 & 35.444 & 0.031 & 1.86 \\
TG 18:0_18:1_18:1 & 35.585 & 35.62 & 0.035 & 2.1 \\
TG 18:0_18:0_18:1 & 36.462 & 36.514 & 0.052 & 3.12 \\
TG 54:2 & 35.616 & 35.647 & 0.031 & 1.86 \\
\hline
\end{tabular}

The objective was to determine whether lipids diluted in $50 \% \mathrm{MeOH}, 50 \% \mathrm{EtOH}$ or $80 \% \mathrm{MeOH}, 20 \% \mathrm{EtOH}$ would produce drastic differences in elution times and chromatography. The retention difference of each species between the two showed insignificant deviations where lipids were accurately assigned. To further validate the results, EIC of two from each lipid class (except DG where only one lipid was identified) were analyzed to determine chromatography. It was concluded that peak shape and resolution of each lipid was acceptable. From this, we assumed a two-fold dilution in $50 \%$ $\mathrm{MeOH}, 50 \% \mathrm{EtOH}$ of lipids extracted from rat livers is compatible with the LC method. This composition was used in all subsequent studies.

Following the development and confirmation of both the gradient method and appropriate sample dilution, the lipid extract was subjected to a final study. The method development for iterative MS/MS acquisition was presented as an alternative to gain 
better insight to low abundant lipid species while providing greater lipidome coverage. This study explores whether iterative MS/MS injections significantly benefits lipidomic coverage for this thesis. Following the lipid extraction protocol as described in Chapter 3.2, lipids extracted from bovine liver was diluted 5-fold in a 1:1 MeOH:EtOH solution. The sample was subsequently injected onto the 6546 QTOF using gradient A (described in Chapter 2.3 and 3.2). A series of single and iterative injections were performed and illustrated in Figure 3.18.

A Iterative Injection

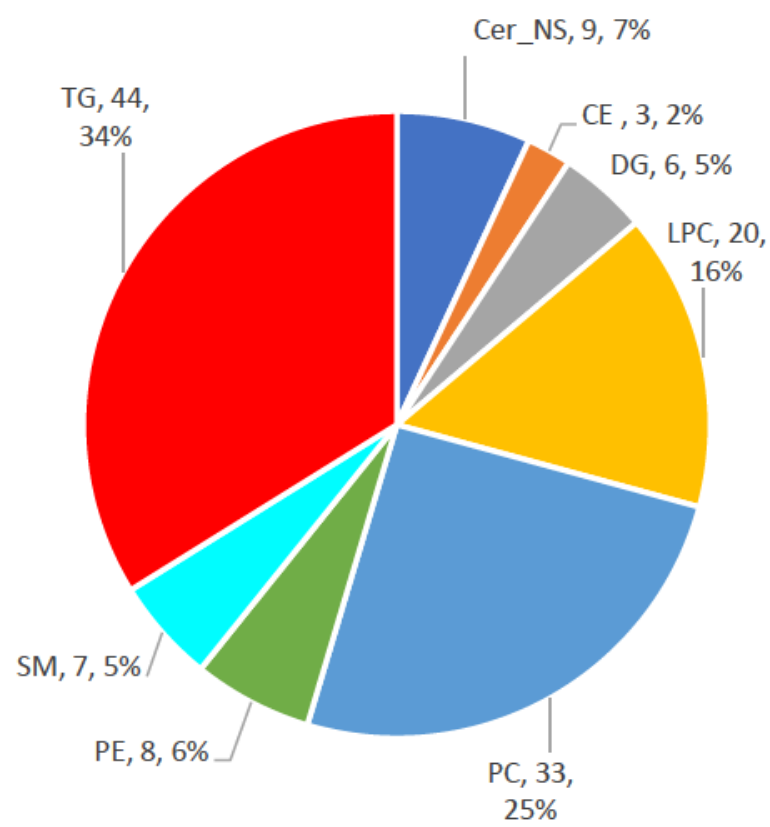

B Single Injection

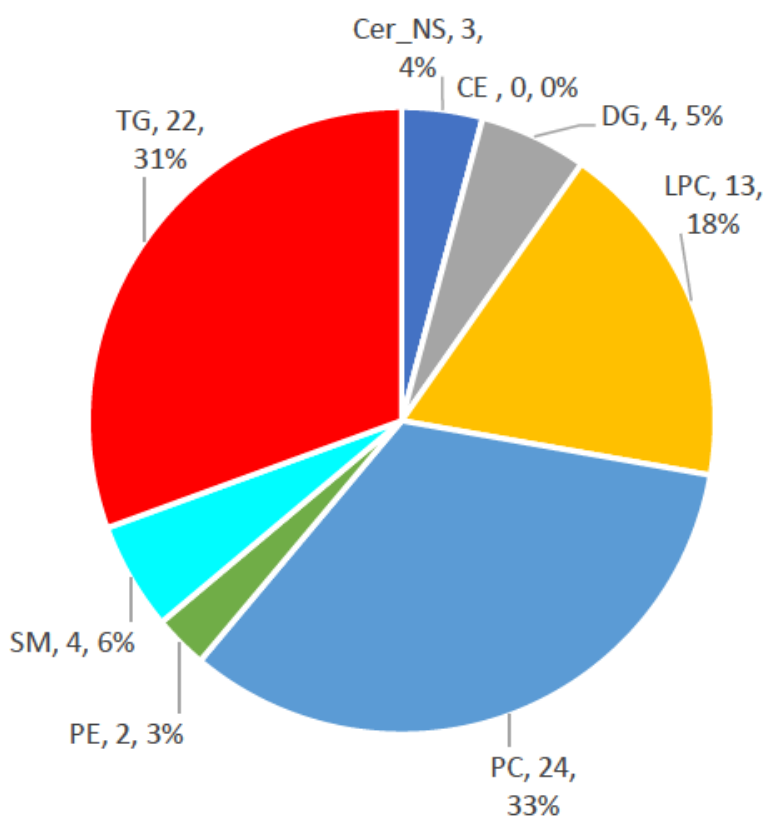

Figure 3.18: Relative distribution of lipids found in bovine liver using gradient program $A$ with $A$ ) iterative injection and $B$ ) single injection

It was determined that iterative injections increased the number of lipids annotated using Lipid Annotator. Compared to a single injection, 6 Cer_NS, 3 CE, 2 DG, 7 LPC, 9 PC, 6 PE, 3 SM, and 22 TG more were annotated. It is not surprising as single injections 
typically select a limited number of precursors at a particular time which results in missing less abundant species like DG. ${ }^{8}$ With iterative MS/MS, continuous analysis of the same sample, excluding previously selected precursors in subsequent injections provide better lipidomic coverage since less abundant species are selected for analysis. One drawback of utilizing iterative is time. With multiple analyses, this results in longer experimental protocols. However, in an untargeted study, gaining better coverage outweighs analysis time since it provides greater insight.

The iterative study was not conducted on rat liver samples due to limited time. However, there is substantial evidence both from this study and literature ${ }^{9}$ that suggests iterative MS/MS will benefit the analysis of lipidomic effects post LDR exposure. Additionally, the number of subsequent injections was not validated and six were adapted from Agilent's application notes. For future work involving any iterative studies, the number of subsequent injections should be tested to determine the point of diminishing returns in the number of lipid species gained.

\subsection{Conclusion}

The results from this chapter illustrated the optimal parameters and conditions used in subsequent experiments presented in Chapter 4. Three LC gradient methods were developed and described. Gradient A was selected as the method of choice since more TGs were identified. Due to the complexity of biological extracts, sample carryover readily occurs because of contamination from lipid samples to the HPLC lines. It was concluded that gradient A minimizes lipid carryover when examined with QC samples (blanks). Sample dilution and sample solution were also studied in this chapter. 
With the development of an effective LC gradient, the composition of the sample solution was investigated to determine whether retention nor chromatography would alter. The different ratios between $\mathrm{MeOH}$ and $\mathrm{EtOH}, 1: 1$ and 4:1 respectively, did not affect either analyte retention or chromatography. It was concluded that a 2 -fold dilution with $50 \% \mathrm{MeOH}$ and $50 \% \mathrm{EtOH}$ for lipid extracts from rat livers is compatible with the gradient method mentioned above. Additionally, the development of an iterative method to acquire more (less abundant) lipids was successful. This was important in developing a proper workflow for accurate untargeted lipidomics experiments. These results enabled the determination of appropriate conditions used in subsequent studies presented in the following chapter.

\subsection{References}

(1) Han, X.; Gross, R. W. Global Analyses of Cellular Lipidomes Directly from Crude Extracts of Biological Samples by ESI Mass Spectrometry: A Bridge to Lipidomics. J. Lipid Res. 2003, 44, 1071-1079. https://doi.org/10.1194/jlr.R300004-JLR200.

(2) Cajka, T.; Fiehn, O. Comprehensive Analysis of Lipids in Biological Systems by Liquid Chromatography-Mass Spectrometry. TrAC Trends Anal. Chem. 2014, 61, 192-206. https://doi.org/10.1016/j.trac.2014.04.017.

(3) Narváez-Rivas, M.; Zhang, Q. Comprehensive Untargeted Lipidomic Analysis Using Core-Shell C30 Particle Column and High Field Orbitrap Mass Spectrometer. J. Chromatogr. A 2016, 1440, 123-134. https://doi.org/10.1016/j.chroma.2016.02.054.

(4) Rustam, Y. H.; Reid, G. E. Analytical Challenges and Recent Advances in Mass Spectrometry Based Lipidomics. Anal. Chem. 2018, 90 (1), 374-397. https://doi.org/10.1021/acs.analchem.7b04836.

(5) Koelmel, J. P.; Ulmer, C. Z.; Jones, C. M.; Yost, R. A.; Bowden, J. A. Common Cases of Improper Lipid Annotation Using High-Resolution Tandem Mass Spectrometry Data and Corresponding Limitations in Biological Interpretation. Biochim. Biophys. Acta BBA - Mol. Cell Biol. Lipids 2017, 1862 (8), 766-770. https://doi.org/10.1016/j.bbalip.2017.02.016. 
(6) Shields, S. W. J.; Canez, C. R.; Wasslen, K. V.; Lee, H.; Stalinski, D.; Trouborst, L.; Joudan, S.; Whitton, S.; Weinert, H. P.; Manthorpe, J. M.; Smith, J. C. Enhancing the Analysis of Complex Lipid Samples Through Developments in Chromatography and Chemical Derivatization. In Molecular Technologies for Detection of Chemical and Biological Agents; Banoub, J. H., Caprioli, R. M., Eds.; NATO Science for Peace and Security Series A: Chemistry and Biology; Springer Netherlands: Dordrecht, 2017; pp 177-206. https://doi.org/10.1007/978-94-024-1113-3_11.

(7) Woodman, M. High-Resolution Analysis of Intact Triglycerides by Reversed Phase HPLC Using the Agilent 1290 Infinity LC UHPLC System. Agilent Techonolgies, 2009.

(8) Koelmel, J. P.; Kroeger, N. M.; Gill, E. L.; Ulmer, C. Z.; Bowden, J. A.; Patterson, R. E.; Yost, R. A.; Garrett, T. J. Expanding Lipidome Coverage Using LC-MS/MS Data-Dependent Acquisition with Automated Exclusion List Generation. J. Am. Soc. Mass Spectrom. 2017, 28 (5), 908-917. https://doi.org/10.1007/s13361-0171608-0.

(9) Koelmel, J. P.; Li, X.; Stow, S. M.; Sartain, M. J.; Murali, A.; Kemperman, R.; Tsugawa, H.; Takahashi, M.; Vasiliou, V.; Bowden, J. A.; Yost, R. A.; Garrett, T. J.; Kitagawa, N. Lipid Annotator: Towards Accurate Annotation in Non-Targeted Liquid Chromatography High-Resolution Tandem Mass Spectrometry (LC-HRMS/MS) Lipidomics Using a Rapid and User-Friendly Software. Metabolites 2020, 10 (101), 20. https://doi.org/10.3390/metabo10030101. 


\section{Chapter 4: Analysis of Low-Dose Radiation Exposed Rat Liver}

\subsection{Introduction}

lonizing radiation $(\mathrm{IR})$ is the high energy emission of particles or waves capable of knocking out electrons which can disrupt homeostasis through the generation of reactive oxygen species (ROS) $\cdot{ }^{1-3}$ This disruption can change intracellular molecules which may result in negative health effects. ${ }^{2}$ An example is lipid peroxidation or the oxidative degradation of lipids. ${ }^{2}$ Lipid peroxidation is associated with ROS as free radicals attack highly unsaturated fatty acids. ${ }^{3-5}$ However, many health risks are highly dependent on dosage level. ${ }^{1,4,6}$ It is evident that exposure to high levels of IR is harmful, however, the biological effects as a result of LDR exposure are less understood. As a result, the definition and speculated biological effects spark controversies. ${ }^{1,7}$ For instance, as discussed in chapter 1.4.2, many studies consider IR under $0.5 \mathrm{~Gy}$ (500 mGy) as LDR ${ }^{1}$, however, publications like Tapio et al. and Wang et al. suggests doses that are $<100$ mGy are defined as LDR. ${ }^{7,8}$ In radiation exposure, what is classified safe is typically the annual dose limits a human may receive. It is at this level where supposedly no physiological effects on a biological system are observed. ${ }^{6}$ However, dose limits are not well defined since assumptions on human health risks are based on the knowledge of radiation cancer risk which is derived from epidemiological studies. ${ }^{9}$

For many years it has been widely accepted that LDR exposure is relatively harmless to humans, however, this may not be true. ${ }^{6}$ The effects of LDR on humans may be subtle but there is growing evidence to suggest that continuous LDR exposure may result in some radiogenic effects. ${ }^{6}$ A study conducted by Little et al. suggests that although LDR may not result in direct cell death, mutations may occur and initiate 
cancerous behaviour. ${ }^{10}$ According to the Canadian Nuclear Safety Commission (CNSC), in Canada, exposure from LDR is mostly contributed from natural background radiation while medical procedures account for the rest. ${ }^{11}$ Aside from medical devices, nuclear workers and uranium miners are also subjected to LDR. It is also evident that prolong exposure increases risks due to cumulative effects. ${ }^{6,11}$

With regards to the field of lipidomics, it is unclear whether LDR exposure enhances or suppresses lipid oxidation. Previous studies suggest that continuous exposure to LDR may implicate significant changes in overall lipid metabolism, especially in the liver. ${ }^{1}$ With the liver as the major organ that maintains homeostasis, synthesizes, and stores highly unsaturated lipids ${ }^{1}$, it is an ideal organ to conduct LDR exposure lipidomics studies. With previous lipidomic studies exploring the effects of IR using a $\gamma$ emitter $\left({ }^{60} \mathrm{Co}\right)^{4,12}$, and a $\beta$ \& $\gamma$ emitter $\left({ }^{137} \mathrm{Cs}\right)^{13,14}$, very little is known about the effects from an $\alpha$-emitting substance. One of the many $\alpha$-emitting materials is ${ }^{210} \mathrm{Po} .{ }^{210} \mathrm{Po}$ is found at low concentrations in the Earth's crust ${ }^{15}$, and although it poses little threat to human health, environmental exposure to ${ }^{210} \mathrm{Po}$ is poorly known. ${ }^{15}$ This calls for much needed research to re-evaluate current regulations of LDR exposure with technologically advanced techniques to protect public health.

In this study, we explore the possible lipidomic effects of IR at different dosage levels on the lipidome through inducing potential lipid peroxidation by $\alpha$-irradiation from ${ }^{210} \mathrm{Po}$ in male Wistar rat livers and measuring relative changes in lipid composition. Furthermore, previous optimizations allow for efficient post-data acquisition of untargeted lipidomics in addition to better overall lipidome coverage. 


\subsection{Experimental}

Sample Preparation: Control, low, medium, and high-dosed rat liver tissues were obtained from collaborative work with Dr. Baki Sadi at Health Canada, Radiation Protection Bureau. The animal experiment was carried out using male Wistar rats at 9 weeks of age. Each Wistar rat weighed approximately $400 \mathrm{~g}$. The rats were purchased from Charles River Laboratories International Inc. (www.criver.com/products-services/findmodel/wistar-igs-rat). Upon arrival, the rats were kept in a metabolic cage from a minimum of 10-14 days prior to the beginning of this experiment to acclimatize to their new surroundings. The rats were kept under a constant temperature of $23 \pm 2^{\circ} \mathrm{C}$. During acclimatization, rats were kept in a 12-hour light and 12-hour dark cycle with free access to rodent chow and reverse osmosis water. All work with the rats were conducted within the Biological Research Facility of the Canadian Nuclear Laboratories (CNL), Chalk River, Ontario, Canada in accordance with the Canadian Council on Animal Care (CCAC) guidelines and policies for performing animal experiments and with an Animal Care Committee (ACC) approved animal protocol. Three groups of Wistar rats (5 per group) were injected via tail vein, under anesthetic, with $45157 \pm 5734 \mathrm{~Bq}, 531454 \pm 41698 \mathrm{~Bq}$ and $1040187 \pm 36252 \mathrm{~Bq}$ of polonium-210, respectively in $1 \%$ sodium citrate. A group of 5 (control) were injected with a similar volume $(0.3 \mathrm{~mL})$ of $1 \%$ sodium citrate. Post injection, all 20 rats were kept in the metabolic cages for 9 days with free access to rodent chow and reverse osmosis water. Overall health, weight, food and water intake, and waste output were monitored daily. On day 10 , end of the exposure period, the rats were euthanized using the CCAC approved method of inhalant anesthetic. Livers were removed from the rats, approximately $1 \mathrm{~g}$ of liver tissue from each rat were stored in a - 
$80^{\circ} \mathrm{C}$. Rat liver tissues were sent to the Radiation Protection Bureau of Health Canada on dry ice for further analysis. Upon arrival, liver tissues were homogenized using a tissue homogenizer and subsampled into multiple tissue homogenates and stored at $-80^{\circ} \mathrm{C}$ for future analysis. One of these subsets were allocated for lipidomics analysis at Carleton University. All tissue samples were kept in Eppendorf tubes. The subset tissue samples were removed from the $-80^{\circ} \mathrm{C}$ freezer and was transported to Carleton University, Carleton Mass Spectrometry Centre on ice. Upon arrival, samples were stored in a $-80^{\circ} \mathrm{C}$ freezer.

Tissue Randomization: Rat liver extractions were done over the course of ten (10) days, once every other day. Each day, a control, a low, a medium and a high-dosed liver tissue were selected at random using Microsoft Excel's randomization function. The order in which of the tissue were extracted was also randomized to avoid biases.

Table 4.1: The tissue sample used for lipid extractions. Sample was randomized by Microsoft Excel's randomization function. Notation as follows: $C=$ control, D1 $=$ Low Dose, D2 = Med Dose, D3 = High Dose. The number after indicates the liver sample.

\begin{tabular}{ccccc}
\hline $\begin{array}{c}\text { Randomization } \\
\text { Ext. Day }\end{array}$ & Control & Low & Med & High \\
\hline Day 1 & C2 & D1-1 & D2-3 & D3-5 \\
Day 2 & C1 & D1-2 & D2-4 & D3-1 \\
Day 3 & C3 & D1-3 & D2-2 & D3-2 \\
Day 4 & C5 & D1-5 & D2-1 & D3-4 \\
Day 5 & C4 & D1-4 & D2-5 & D3-3 \\
\hline
\end{tabular}

Lipid Extraction: See Chapter 2.2

Sample Pooling: See Chapter 2.2 
Sample Randomization: A total of 36 MS-only injections were done each day, representing technical triplicate injections per extraction. The order of the 36 MS-only injections were randomized using Microsoft Excel's randomization function to avoid any biases.

Reversed phase high performance liquid chromatography: See Chapter 2.3

HLPC-MS/MS: See Chapter 2.3

Data Analysis: See Chapter 2.4 and Chapter 2.5

\subsection{Results and Discussion}

After analysis of rat liver samples, peak areas (obtained from Profinder) of each triplicate injection were averaged, resulting in an averaged peak area for each liver extraction. Each identified lipid was standardized to the internal standard, LPC C13:0, according to Chapter 2.5. Although the target mass of each tissue sample was $1 \mathrm{mg}$, it was extremely difficult to accurately weigh such a small value. Some of the rat liver tissues provided did not contain enough to aliquot $1 \mathrm{mg}$ of wet tissue weight into triplicate trials. Therefore, lipids were normalized to each wet tissue sample measured from each extraction (see Chapter 2.5). The mass of each aliquot was recorded for this purpose and listed in Table 4.2.

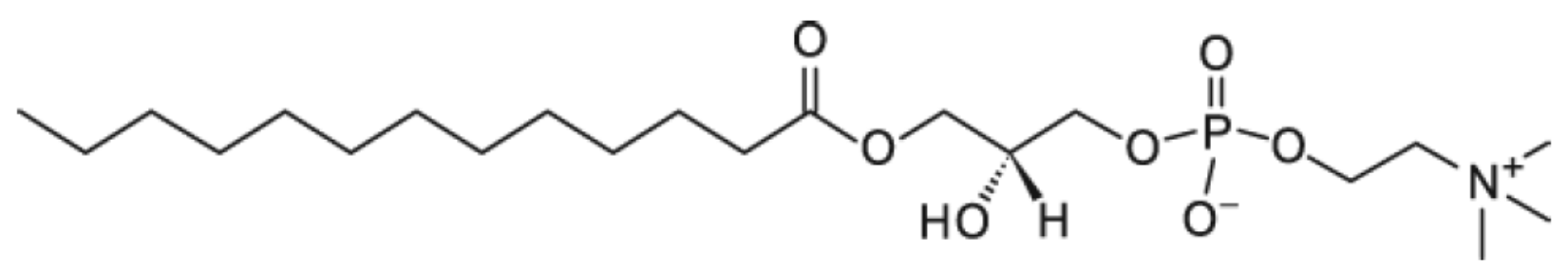

Figure 4.1: Structure of internal standard LPC C13:0 (453.29 Da). 
Table 4.2: Mass of wet liver tissue (in $\mathrm{mg}$ ) used in each extraction. Target mass was 1 $\mathrm{mg}$. However, since some samples provided did not contain a total of $3 \mathrm{mg}$ (wet tissue weight), therefore some recorded masses are lower than target weigh. Liver tissues were weighed on a Mettler AT250 analytical balance.

\begin{tabular}{ccccc}
\multicolumn{6}{l}{ CONTROL } \\
\hline Rat \# & Day \# & Extraction 1 $(\mathbf{m g})$ & Extraction 2 $(\mathbf{m g})$ & Extraction 3 (mg) \\
\hline Rat 1 & 2 & 0.99 & 0.91 & 0.88 \\
Rat 2 & 1 & 1.09 & 1.03 & 0.98 \\
Rat 3 & 3 & 0.86 & 0.63 & 0.20 \\
Rat 4 & 5 & 0.99 & 1.21 & 0.82 \\
Rat 5 & 4 & 0.98 & 0.53 & 0.73 \\
\hline
\end{tabular}

\begin{tabular}{ccccc}
\multicolumn{6}{l}{ LOW DOSE } \\
\hline Rat \# & Day \# & Extraction 1 (mg) & Extraction 2 (mg) & Extraction 3 (mg) \\
\hline Rat 1 & 1 & 0.93 & 0.51 & 1.07 \\
Rat 2 & 2 & 1.17 & 1.05 & 0.90 \\
Rat 3 & 3 & 1.02 & 1.25 & 1.00 \\
Rat 4 & 5 & 1.15 & 1.15 & 0.98 \\
Rat 5 & 4 & 0.80 & 1.00 & 0.84 \\
\hline
\end{tabular}

MEDIUM DOSE

\begin{tabular}{ccccc}
\hline Rat \# & Day \# & Extraction 1 $(\mathbf{m g})$ & Extraction 2 $(\mathbf{m g})$ & Extraction 3 $(\mathbf{m g})$ \\
\hline Rat 1 & 4 & 1.04 & 1.05 & 1.20 \\
Rat 2 & 3 & 1.04 & 1.01 & 0.84 \\
Rat 3 & 1 & 1.01 & 0.51 & 0.24 \\
Rat 4 & 2 & 1.03 & 1.25 & 0.90 \\
Rat 5 & 5 & 1.18 & 0.99 & 1.00 \\
\hline
\end{tabular}

HIGH DOSE

\begin{tabular}{ccccc}
\hline Rat \# & Day \# & Extraction 1 $(\mathbf{m g})$ & Extraction 2 $(\mathbf{m g})$ & Extraction 3 $(\mathbf{m g})$ \\
\hline Rat 1 & 2 & 0.85 & 0.94 & 0.80 \\
Rat 2 & 3 & 0.65 & 1.13 & 1.09 \\
Rat 3 & 5 & 0.84 & 0.26 & 0.39 \\
Rat 4 & 4 & 1.04 & 1.10 & 1.09 \\
Rat 5 & 1 & 1.08 & 0.38 & 0.57 \\
\hline
\end{tabular}

To demonstrate the quality of the data acquired and the accuracy of the 6546 QTOF, a non-extracted internal standard sample, $0.7 \mu \mathrm{M}$ of LPC C13:0 (Figure 4.1) in $\mathrm{MeOH}$, was injected every 10 samples, acquiring in MS only mode (see Figure 2.2). An 
EIC of $454.29 \mathrm{Da},[\mathrm{M}+\mathrm{H}]^{+}$of LPC C13:0, was performed using Agilent's MassHunter Qualitative Analysis (Version 10.0). LPC C13:0 was chosen as the internal standard since it is not natively present in mammalian cells. ${ }^{16,17}$ Figure 4.2 illustrates every non-extracted internal standard injection that occurred throughout the experiment. In addition, a matrix spiked sample, consisting of $42 \mu \mathrm{L}$ of $10 \mu \mathrm{M}$ LPC C13 and no tissue, was prepared the same way as described in Chapter 2.2 and acquired in MS only mode. The purpose was to determine any sample matrix effects as well as relative extraction recoveries. Since both the Quat-pump Infinity II 1260 and the 6545 QTOF were operated for an extended amount of time (over 12 continuous days), this was done to ensure both retention time and peak intensity of a known analyte was consistent. Figure 4.3 illustrates all 5 spiked samples with triplicate injections of each to determine the quality of the data acquired on the 6545 LC-QTOF.

Post data acquisition, the chromatograms in Figure 4.2 and Figure 4.3 showed two peaks. This was expected as the manufacturer, Avanti Lipids, states on their product page that the standard may contain up to $10 \%$ of the 2 -LPC isomer. The isomer of interest, LPC 13:0/0:0, had a retention time range between 4.623 to $4.712 \mathrm{mins}, \Delta=0.089 \mathrm{mins}$, or 5.34 seconds, which is well within the retention time tolerance set in Agilent's Profinder (retention time tolerance $= \pm 0.15$ mins, see Chapter 2.4.4) to be able to quantify the correct peak. Similarly, the spiked samples also contained two peaks, which were fully identified as two isomers of LPC C13:0. The retention time range for the isomer of interest was between 4.644 to 4.706 mins, $\Delta=0.062$ mins, or 3.72 seconds. Given the negligible retention time shift across the course of the experiment, the data obtained required minor retention time adjustments in Profinder. 


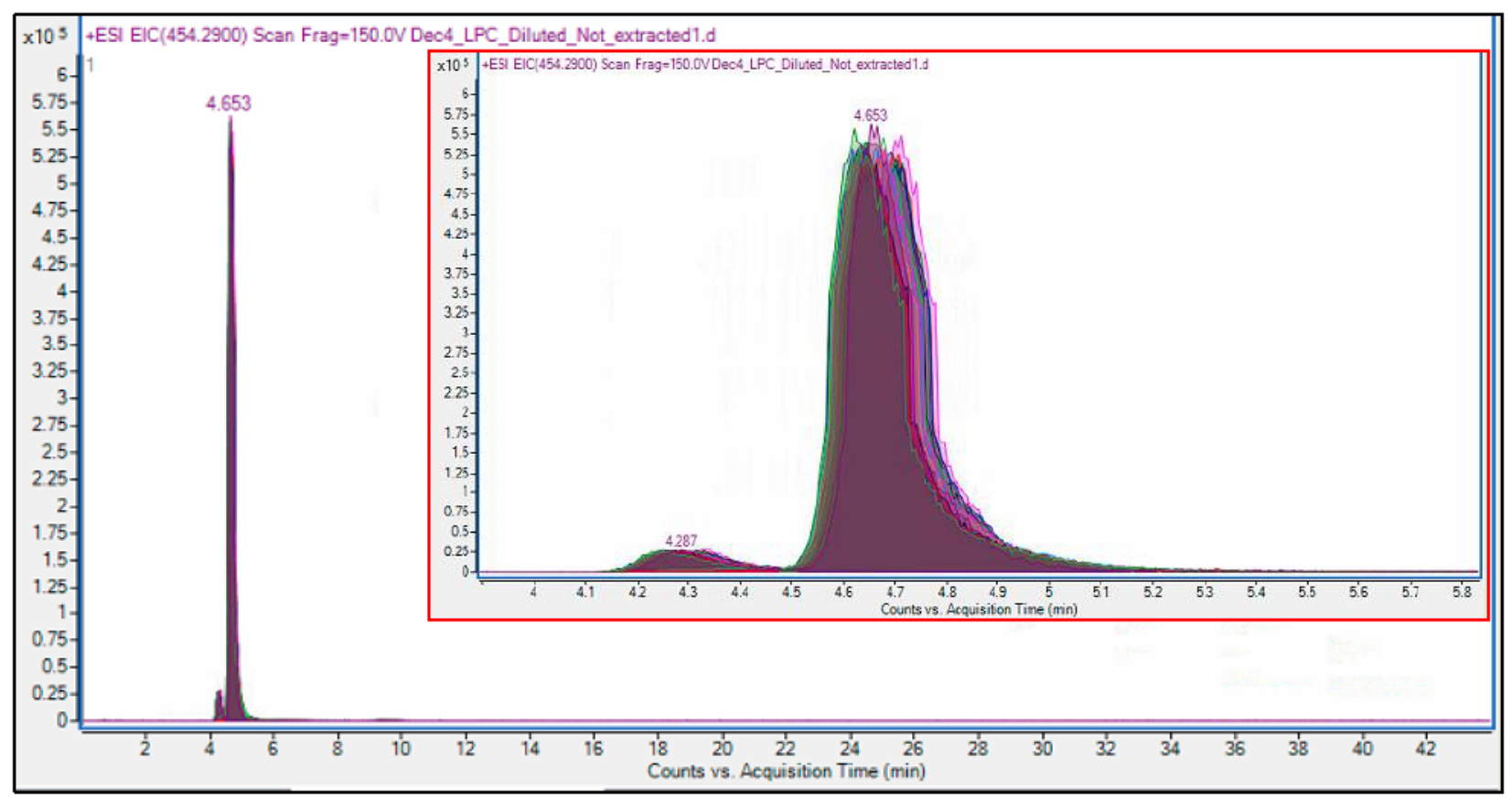

Figure 4.2: Extracted ion chromatogram of $\mathrm{m} / \mathrm{z} 454.2940$, [M+H] ${ }^{+}$of LPC 13:0/0:0 (internal standard) from twenty nine $5 \mu \mathrm{L}$ injections of $0.7 \mu \mathrm{M}$ internal standard.

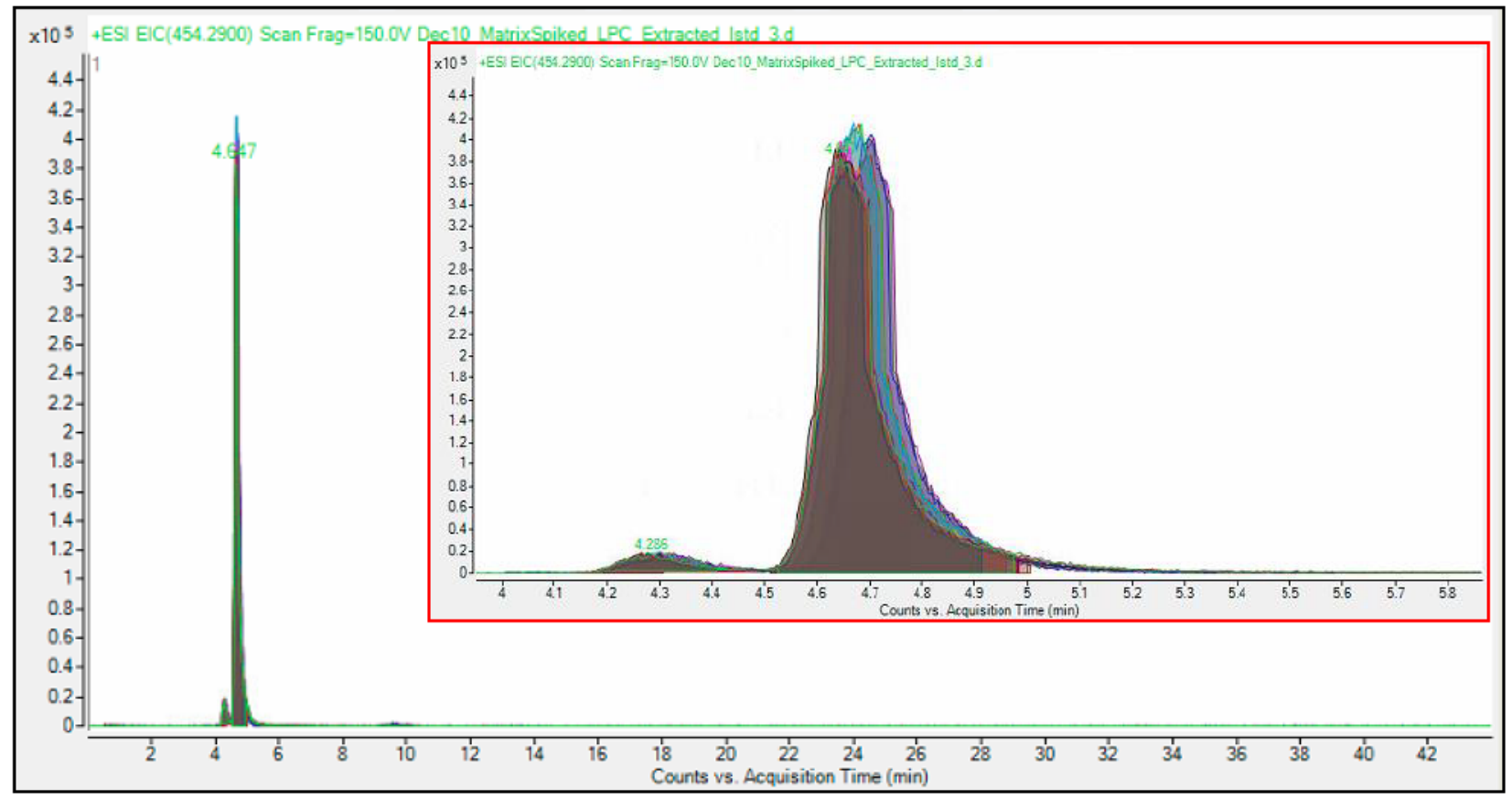

Figure 4.3: Extracted ion chromatogram of $\mathrm{m} / \mathrm{z} 454.2940,[\mathrm{M}+\mathrm{H}]^{+}$of LPC 13:0/0:0 (internal standard) from fifteen $5 \mu \mathrm{L}$ injections (5 extracted spiked samples, each in triplicate). 
A total of 214 lipids were identified over the 20 rat liver samples. The liver samples yielded 9 classes of lipids including 62 PCs, 11 LPCs, 6 CEs, 2 ceramides, 1 CL, 6 DGs, 105 TGs, 6 SMs, and 15 PEs. The overall results are summarized in Figure 4.4. The rat liver tissues contained mostly TGs, followed by PCs, PEs, LPCs, CEs, DGs. SMs, Cer_NS and CL.

\section{Overall Distribution of 214 Lipids in Rat Liver}

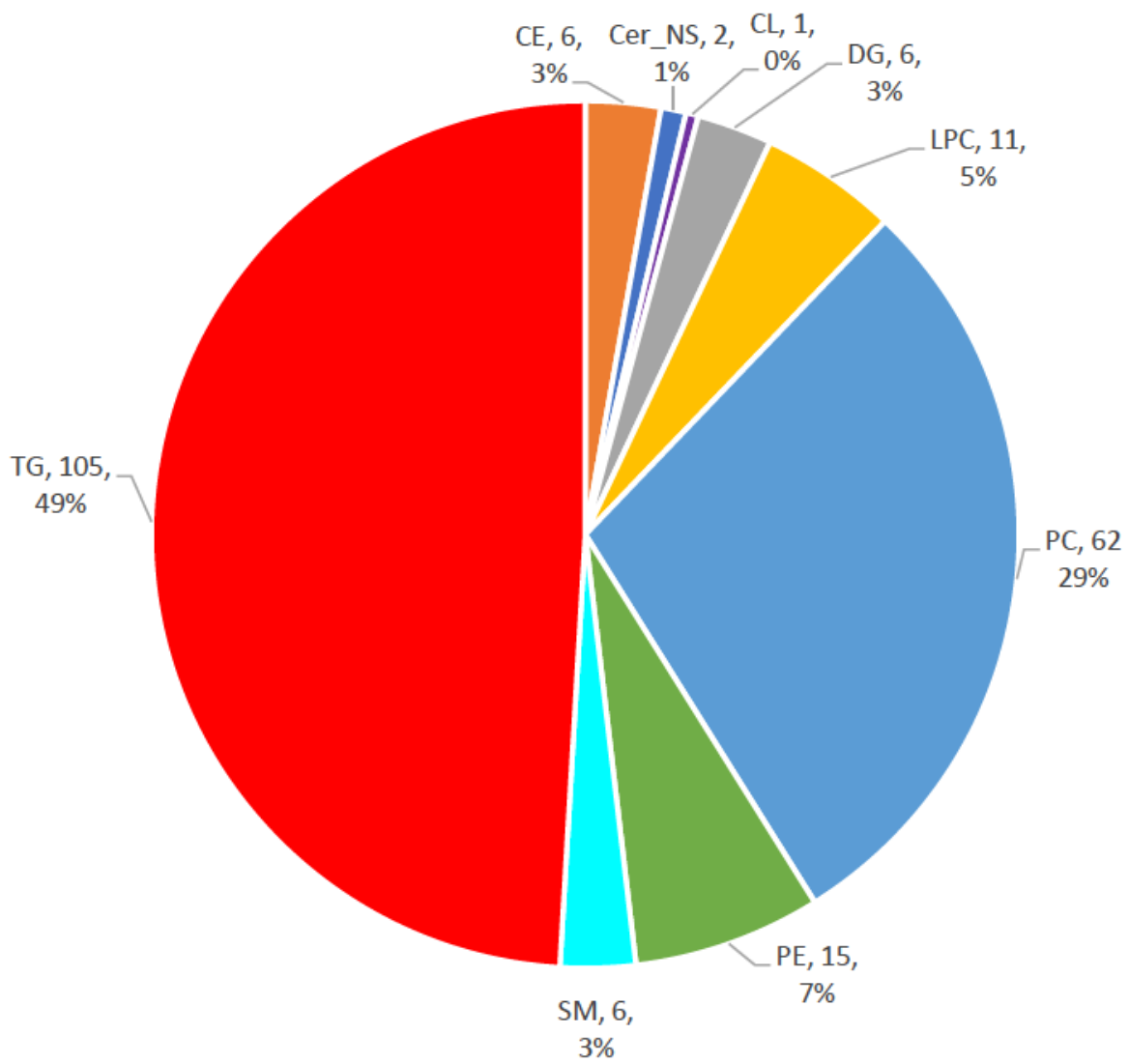

Figure 4.4: Distribution of lipid classes found in rat liver across all four dosage categories. There are a total of 214 lipids found in 20 rat liver samples that were fully identified by Agilent's Lipid Annotator and quantified using Agilent's Profinder.

In addition to normalizing to the internal standard, standardized area values were determined followed by calculating $p$-values $(\alpha=0.05)$ using a student's t-test to identify significant changes for each lipid in each dosage category. To avoid potential false 
discoveries and increase confidence in our results, a false discovery rate (FDR) correction using the Benjamini-Hochberg (BH) method was applied with $\alpha=0.01$ (See Chapter 2.5). It was found that $19.6 \%$ of the lipids did incur statistically significant changes when monitoring the dynamics of these lipid species between the high dose versus control tissues. $17.3 \%$ of the lipids changed significantly between medium dose versus control and $9.3 \%$ of the lipids significantly changed between low dose versus control.

In this study, the normalized dataset for ESI+ mode was processed through RStudio (Version 4.0) to generate a heatmap, Figure 4.5, and a standard principal component analysis (PCA), Figure 4.7. From Figure 4.5, an observation was made. The data illustrates hierarchical clustering from samples that are in different dosage categories with a few triplicates not grouped. Most of the biological triplicates from the same high dose IR rat livers, labelled orange, were grouped. However, there is one outlier where the first triplicate from high dose liver 3 (High3_1) was not grouped with the rest of High3 extractions. This non-grouping can also be seen with the first triplicate in medium dose liver 3 (Mid3_1), first triplicate in low dose liver 3 (Low3_1), first triplicate in low dose liver 1 (Low1_1) as well as a few control rat liver extractions (Crt|3_3, Crt|2_2, Crt|3_2, Crt|3_1, and Crt|5_1). Further investigations revealed that High3_1, Mid3_1, Crt|3_3, and Crt|5_1 all consisted of tissues masses that were different from their respective triplicates. For example, the tissue mass of High3_ 1 is $0.84 \mathrm{mg}$ compared to $0.26 \mathrm{mg}$ and $0.39 \mathrm{mg}$ from High3_2 and High3_3 respectively. This may result in a different concentration of lipids in one biological triplicate compared to the other two biological extractions. When comparing Low3_1 to the other two biological triplicates (Low3_2 and Low3_3), many of the same lipids do show the same pattern. This could be a result of lipids randomly changing since 
the lipidome is highly dynamic. This may also apply to samples Crtl2_2 and Low1_1 with their biological triplicate. However, when comparing to Crtl_3, all three analyses were different. If the hierarchical clustering from the samples were removed and rearranged such that samples were organized by their dosage level, Figure 4.6, no distinct pattern between the dosage levels emerges.

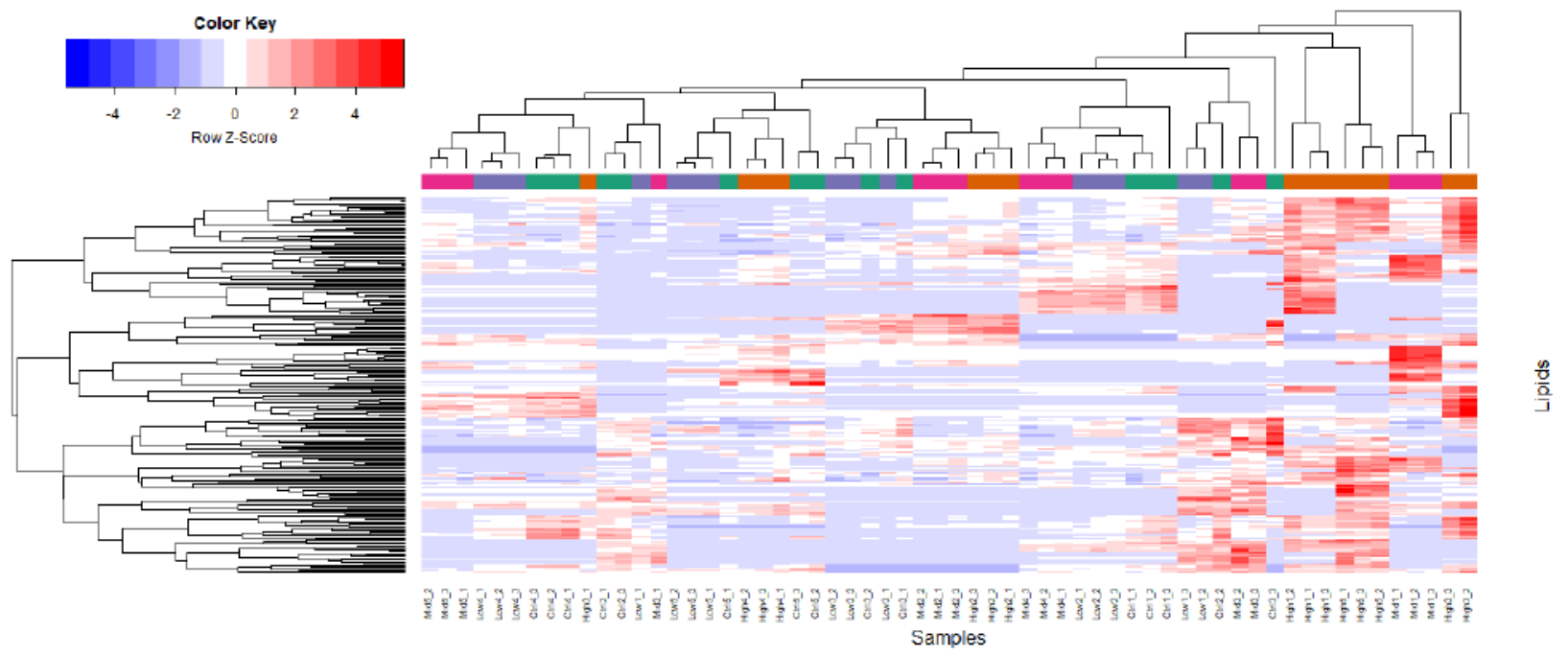

Figure 4.5: Heatmap with hieratical clustering of all the rat livers and lipid species identified in this experiment. Colours on the top represent dosage levels (control = green, low $=$ purple, medium $=$ magenta , and high $=$ orange $)$.
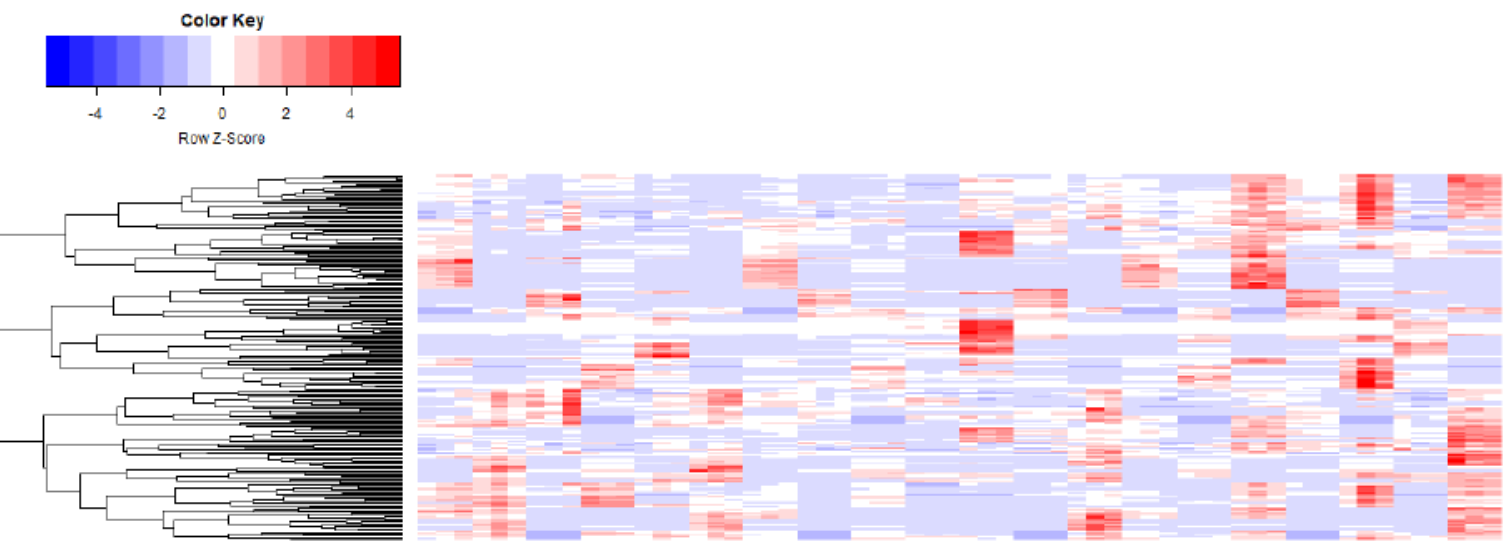

Figure 4.6: Heatmap of all the rat livers used in this experiment without hierarchical clustering of the samples. Samples were organized from control, low, medium to high dosage. 
An overview demonstrating the dynamic change between each dosage level can be observed in Figures 4.7 and 4.8. Figure 4.7 shows significant lipid changes in the high dosage level compared to the control, low, and medium dose levels, after applying the BH method FDR correction. In addition, a relative abundance of fully identified lipids with normalized peak area averages in all rat livers is illustrated in Supplementary 1-8. The PCA illustrated in Figure 4.7 describes $40.6 \%$ of the variation in this study. Each data point represents the overall variations found in each liver extract conducted in this work. It is evident that many control, low and medium extracts are clustered together along the Dim1 (dimension 1) axis. While High4 has similar variations with controls, low and medium groups, it is noteworthy that the majority of the high dose extracts are separate. With the coloured ovals depicting the $95 \%$ confidence ellipses, much of the dataset does

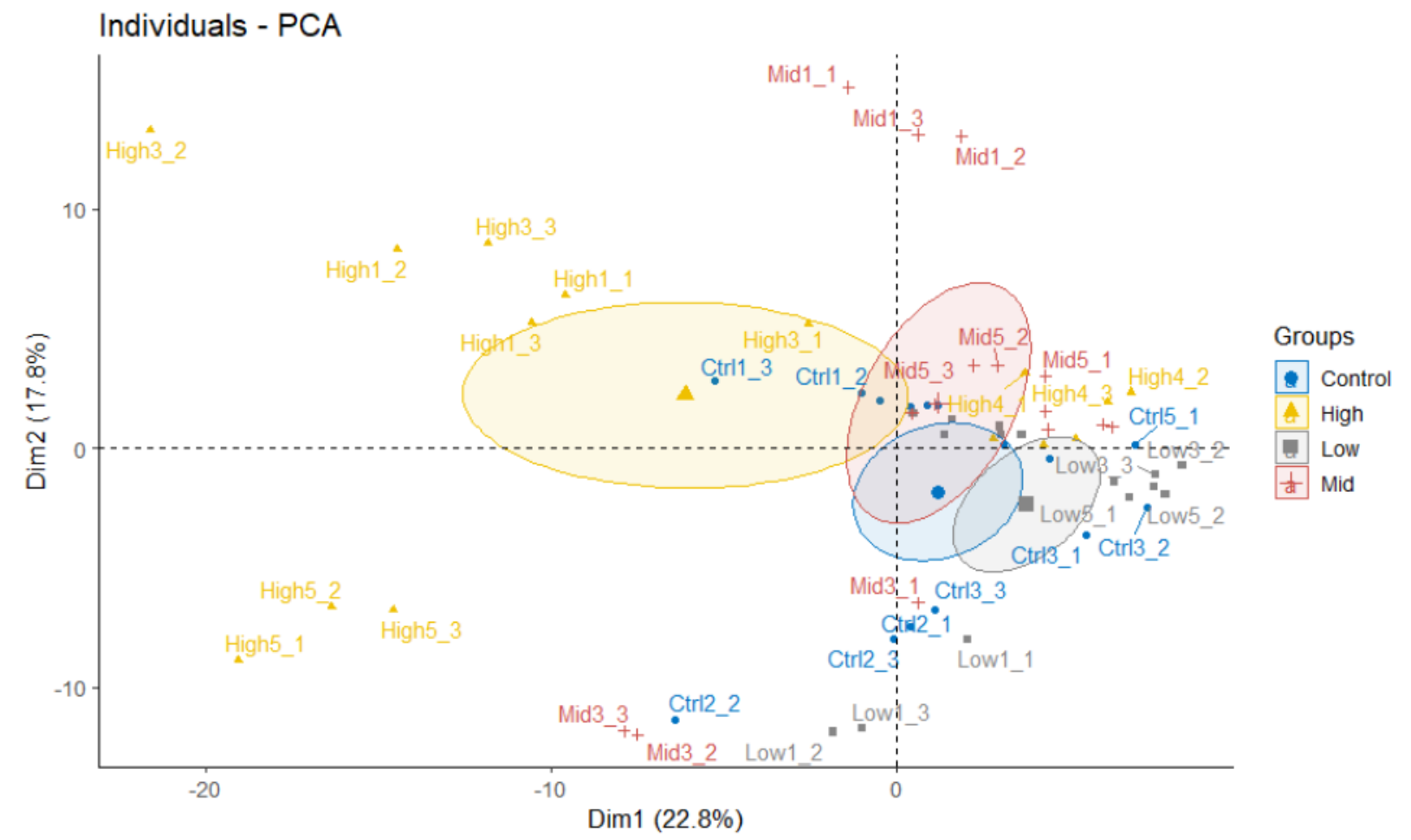

Figure 4.7: PCA of all the rat livers used in this experiment. PCA describes $40.6 \%$ of the variation in this study. The ellipses represent $95 \%$ confidence with respect to the dosage group. 
not fall in their respective ellipses however, it is evident that there are no overlaps between the high and control samples. Additionally, there are no overlaps between the low and medium $95 \%$ confidence ellipses. Based on the results, we can conclude that the high dose and controls are not relatively similar to each other, and the control samples are relatively similar to the low and medium doses.

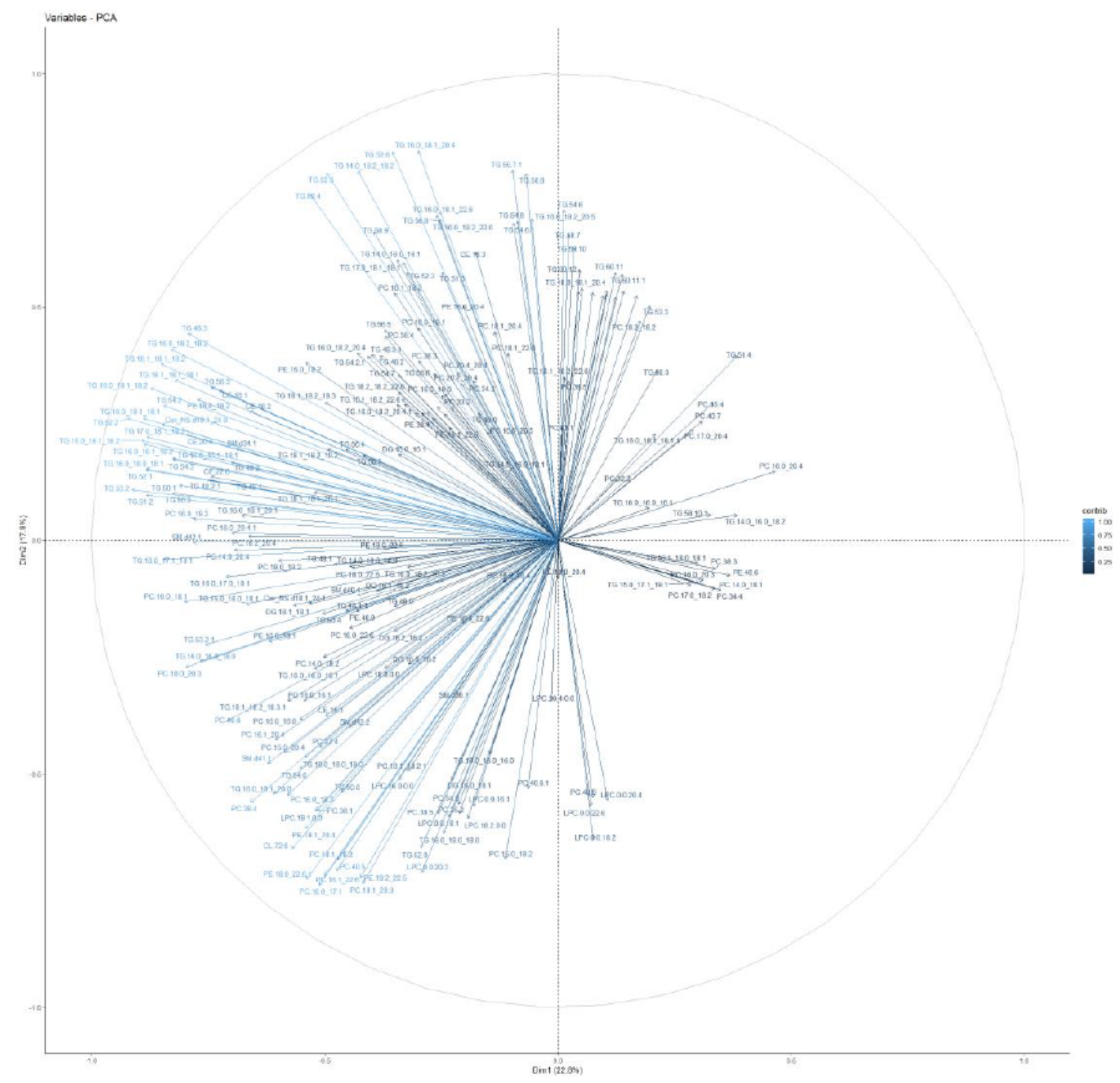

Figure 4.8: Correlation circle of all the lipids annotated from in this experiment. Magnitude of the arrow and colour illustrates correlations of each lipid to each of the two principal components. 
Damage of the lipidome as a direct result of radiation exposure is well established. ${ }^{1-3}$ In the literature, several lipids exhibit significant changes in serum postirradiation analysis. ${ }^{2-4}$ Initial comparisons were first made between the high dose rat livers and the control rat livers to determine potential significant changes. Our hypothesis follows previous literature studies where some lipids would change ${ }^{1-3}$, as a result of radiation exposure. However, the effects of low dose radiation on a liver lipidome were poorly known. Following normalization of the data, a Manhattan plot was created using RStudio to illustrate the lipids that significantly changed as a result of LDR compared to none. Figure 4.9 illustrates many lipids that significantly changed, indicated by the points above the red horizontal line (FDR adjusted p-value). Unfortunately, not all lipids were labelled in RStudio.

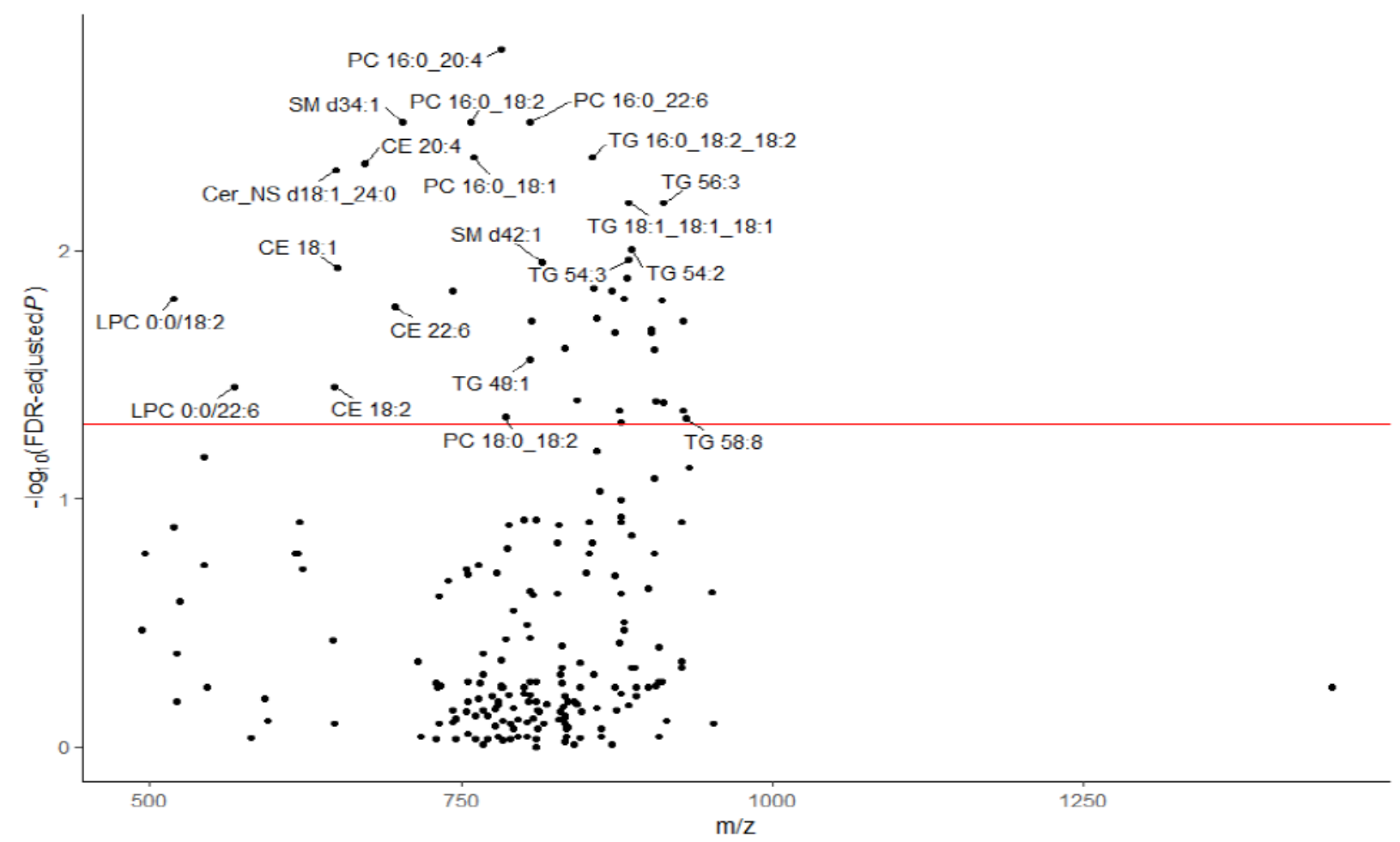

Figure 4.9: Manhattan plot depicting fold change between the high versus control. Red line indicates the statistically significant line after applying the $\mathrm{BH}$ method. X-axis indicates $\mathrm{m} / \mathrm{z}$. 
Additionally, a volcano plot (Figure 4.10) was created to illustrate the fold change each lipid experienced between the high dose and control groups and whether it was significant. Of the $19.6 \%$ of lipid species that showed significant changes previously in the Manhattan plot, $15.9 \%$ showed a significant increase meanwhile, $2.3 \%$ showed a significant decrease. Of the many lipids that increased, illustrated by the red vertical line at $\log _{10}(F C)=0.5$, the majority of the lipids were TGs, with some significant changes towards CEs, PCs, and SMs. Lipids that experience significant decrease include LPCs and a few TGs. $15.9 \%$ of lipids that were found to significantly increase because of radiation exposure are summarized in Table 4.3 and $2.3 \%$ of lipids that significantly decreased are summarized in Table 4.4 .

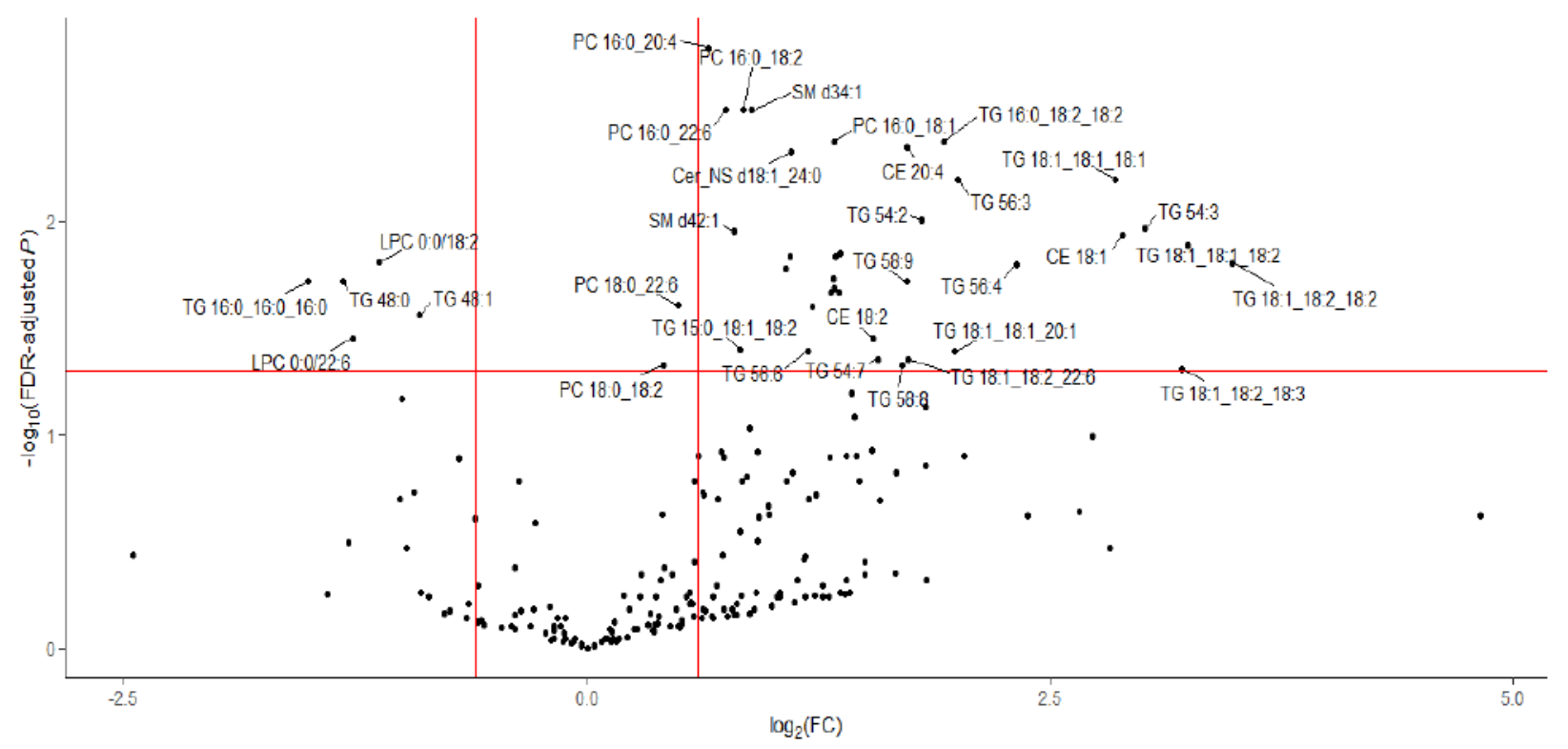

Figure 4.10: Volcano plot depicting fold change between the high versus control. Red horizontal line indicates the statistically significant line after applying the $\mathrm{BH}$ method. Red vertical lines indicate $\log _{10}(\mathrm{FC})= \pm 0.5$.

Table 4.3: Lipids showing significant increase in fold change between the high dosed livers versus control livers.

\begin{tabular}{cccccc}
\hline Compound Name & p_val & FC & Avg. m/z & fdr.adj.p_val & ex.thres \\
\hline CE 18:2 & 0.00554738 & 2.91340785 & 648.58528 & 0.03535574 & TRUE \\
\hline
\end{tabular}




\begin{tabular}{|c|c|c|c|c|c|}
\hline CE $18: 1$ & 0.00076764 & 7.40349959 & 650.60092 & 0.01173385 & TRUE \\
\hline CE 20:4 & 0.00014707 & 3.31129608 & 672.58536 & 0.00449617 & TRUE \\
\hline CE 22:6 & 0.00172716 & 2.09891209 & 696.58498 & 0.0168006 & TRUE \\
\hline Cer_NS d18:1_24:0 & 0.00017709 & 2.14602623 & 649.63818 & 0.00473726 & TRUE \\
\hline SM d34:1 & $5.24 \mathrm{E}-05$ & 1.85340968 & 702.56834 & 0.00303345 & TRUE \\
\hline SM d42:1 & 0.00068226 & 1.73745281 & 814.69356 & 0.01123097 & TRUE \\
\hline PE 18:0_18:2 & 0.00122368 & 2.14074724 & 743.54932 & 0.01459519 & TRUE \\
\hline PC 16:0_18:2 & 5.67E-05 & 1.79392134 & 757.5639 & 0.00303345 & TRUE \\
\hline PC 16:0_18:1 & 0.00010784 & 2.52761886 & 759.57938 & 0.00423074 & TRUE \\
\hline PC 16:0_20:4 & $7.23 \mathrm{E}-06$ & 1.57986161 & 781.5642 & 0.00154722 & TRUE \\
\hline PC 18:0_18:2 & 0.00878853 & 1.3302422 & 785.59512 & 0.04701864 & TRUE \\
\hline PC 16:0_22:6 & $3.44 \mathrm{E}-05$ & 1.68050506 & 805.5636 & 0.00303345 & TRUE \\
\hline PC 18:0_22:6 & 0.00348262 & 1.40973638 & 833.5942 & 0.02484266 & TRUE \\
\hline TG 16:0_18:2_18:2 & 0.00011862 & 3.8011833 & 854.73796 & 0.00423074 & TRUE \\
\hline TG 16:0_18:1_18:2 & 0.00106119 & 2.57575008 & 856.75412 & 0.01419344 & TRUE \\
\hline TG 17:0_18:1_18:2 & 0.00122763 & 2.53578743 & 870.76796 & 0.01459519 & TRUE \\
\hline TG 53:2 & 0.00282133 & 2.57490888 & 872.7838 & 0.02154266 & TRUE \\
\hline TG 54:7 & 0.00794837 & 2.96546619 & 876.721225 & 0.04424993 & TRUE \\
\hline TG 18:1_18:2_18:3 & 0.00967068 & 9.25632784 & 878.736067 & 0.0492744 & TRUE \\
\hline TG 18:1_18:2_18:2 & 0.00146999 & 11.1876057 & 880.7526 & 0.01572893 & TRUE \\
\hline TG 18:1_18:1_18:2 & 0.00090568 & 9.44871482 & 882.76845 & 0.01292105 & TRUE \\
\hline TG 18:1_18:1_18:1 & 0.00027994 & 7.22009988 & 884.78428 & 0.00640352 & TRUE \\
\hline TG 54:3 & 0.00061173 & 8.06843052 & 884.784425 & 0.0109092 & TRUE \\
\hline TG 54:2 & 0.00050688 & 3.50013688 & 886.7998 & 0.0098612 & TRUE \\
\hline TG 16:0_18:2_22:6 & 0.00291933 & 2.49312365 & 902.73742 & 0.02154266 & TRUE \\
\hline TG 56:8 & 0.0026059 & 2.5235013 & 902.73742 & 0.02065419 & TRUE \\
\hline TG 16:0_18:1_22:6 & 0.00362719 & 2.32685181 & 904.75332 & 0.02503933 & TRUE \\
\hline TG 56:6 & 0.00682685 & 2.28033525 & 906.76805 & 0.04058183 & TRUE \\
\hline TG 56:4 & 0.00156362 & 4.97499701 & 910.7991 & 0.01593404 & TRUE \\
\hline TG 18:1_18:1_20:1 & 0.00706997 & 3.95756799 & 912.814925 & 0.0408912 & TRUE \\
\hline TG 56:3 & 0.00029923 & 4.0094823 & 912.81502 & 0.00640352 & TRUE \\
\hline TG 58:9 & 0.00230481 & 3.30546528 & 928.752125 & 0.0192858 & TRUE \\
\hline TG 18:1_18:2_22:6 & 0.00806424 & 3.32203344 & 928.7524 & 0.04424993 & TRUE \\
\hline TG $58: 8$ & 0.00910703 & 3.25130854 & 930.767775 & 0.04753424 & TRUE \\
\hline
\end{tabular}

p_value indicated the value from t-test using $\alpha=0.05$, FC indicates the fold change, determined by taking the averaged normalized intensity from the high dose divided by the averaged normalized intensity from the control. Avg.mz is the average experimental $\mathrm{m} / \mathrm{z}$. fdr.adj.p_value is the $\mathrm{p}$ - value after using the $\mathrm{BH}$ method for false discovery rate. Ex.thres is the output from RStudio indicating whether the lipid species does accrue significant change. True indicating significant change. 
Table 4.4: Lipids showing significant decrease in fold change between the high dosed livers versus control livers.

\begin{tabular}{cccccc}
\hline Compound Name & p_val & FC & Avg. $\mathrm{m} / \mathrm{z}$ & fdr.adj.p_val & ex.thres \\
\hline LPC 0:0/18:2 & 0.00138752 & 0.45855047 & 519.3331 & 0.01562785 & TRUE \\
LPC 0:0/22:6 & 0.00561727 & 0.41630505 & 567.33272 & 0.03535574 & TRUE \\
TG 16:0_16:0_16:0 & 0.00219155 & 0.35255923 & 806.73725 & 0.0192858 & TRUE \\
TG 48:0 & 0.00234314 & 0.40149012 & 806.737 & 0.0192858 & TRUE \\
TG 48:1 & 0.00414016 & 0.53499811 & 804.72168 & 0.02768732 & TRUE \\
\hline
\end{tabular}

p_value indicated the value from t-test using $\alpha=0.05$, FC indicates the fold change, determined by taking the averaged normalized intensity from the high dose divided by the averaged normalized intensity from the control. Avg.mz is the average experimental $\mathrm{m} / \mathrm{z}$. fdr.adj.p_value is the $\mathrm{p}$ - value after using the $\mathrm{BH}$ method for false discovery rate. Ex.thres is the output from RStudio indicating whether the lipid species does accrue significant change. True indicating significant change.

Figures 4.11 and 4.12 presents a comparison between the control and high dose normalized peak area averages of the lipids that increased significantly. The low and medium dose levels were included to illustrate the changes in between. This includes 6 PCs, 22 TGs, 2 SMs, 1 PE, 1 Cer_NS, and 4 CEs. The comparison of the 10 highest normalized peak area averages is presented in Figure 4.11 while the remaining 16 lipids are presented in Figure 4.12. Additionally, Figure 4.13 presents a comparison between the control, low, medium, and high dose normalized peak area averages of the lipids that decreased significantly. This includes 2 LPCs and 3 TGs. 


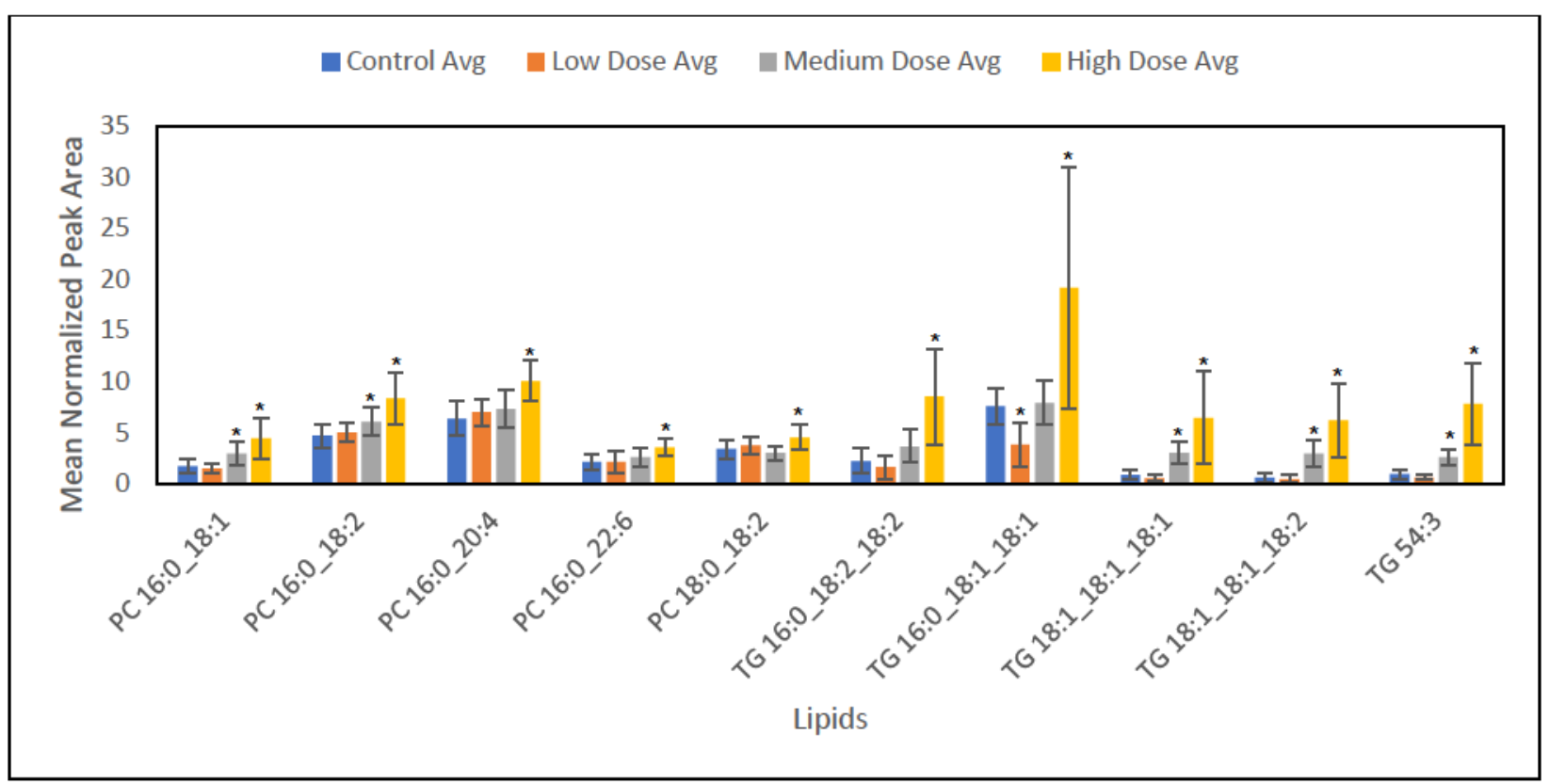

Figure 4.11: Relative abundance of the top 10 lipids with the highest normalized peak area averages in rat liver control (blue) versus high (yellow) dose samples that increased in a statistically significant manner after applying an FDR correction. Low (orange) and medium (grey) dose levels were included to show the change in between the control and high dosage.

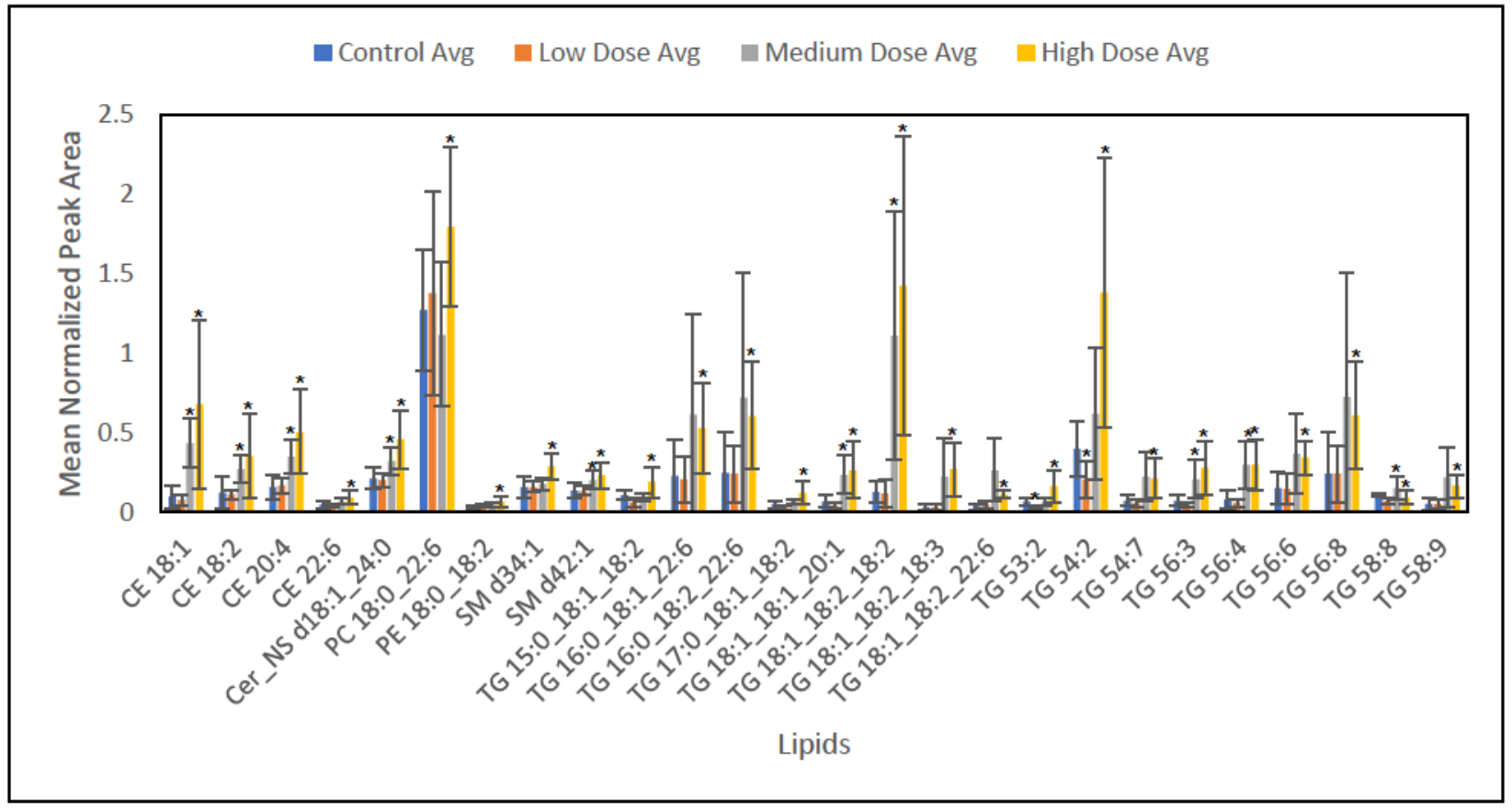

Figure 4.12: Relative abundance of the 26 lipids with normalized peak area averages in rat liver control (blue) versus high (yellow) dose samples that increased in a statistically significant manner after applying an FDR correction. Low (orange) and medium (grey) dose levels were included to show the change in between the control and high dosage. 


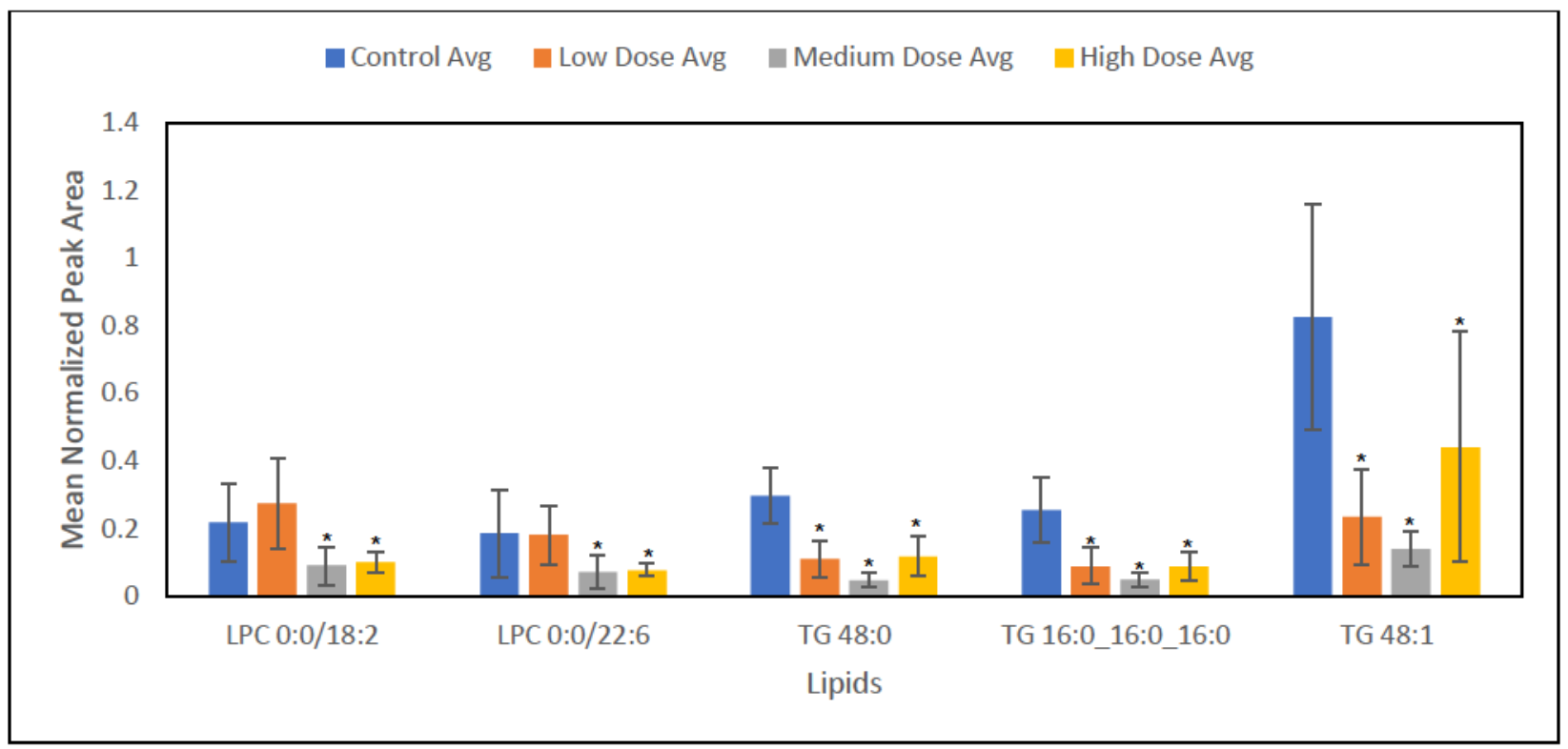

Figure 4.13: Relative abundance of 5 lipids with normalized peak area averages in rat liver control (blue) versus high (yellow) dose samples that decreased in a statistically significant manner after applying an FDR correction. Low (orange) and medium (grey) dose levels were included to show the change in between the control and high dosage.

A similar analysis was done when comparing the medium dose livers to the controls. A Manhattan plot was created and like Figure 4.9, Figure 4.14 reveals a significant amount of lipids above the statistical significance line. Additionally, a volcano plot (Figure 4.15) was created to illustrate the fold change each lipid experienced and whether it was significant. Of the $17.3 \%$ of lipid species that showed significant changes previously in the Manhattan plot, $7.5 \%$ showed a significant increase meanwhile, $9.8 \%$ showed a significant decrease. Of the many lipids that increased, illustrated by the red vertical line at $\log _{10}(F C)=0.5$, the majority of the lipids were TGs, with some significant changes towards CEs and, PCs. Lipids that experience significant decrease include LPCs, many TGs, and DG. $7.5 \%$ of lipids that were found to significantly increase because of radiation exposure are summarized in Table 4.5 and $9.8 \%$ of lipids that significantly decreased are summarized in Table 4.6. Figures 4.16 and 4.17 presents a comparison between the control and medium normalized peak area averages of the lipids that 
increased significantly. The low and high dose levels were included to illustrate any changes occurring at those levels. This includes 2 PCs, 8 TGs, 1 SMs, 1 PE, 1 Cer_NS, and $3 \mathrm{CEs}$. It is noteworthy that all lipids species that experienced a statistically significant normalized area increase after a medium dose was present in the high dose as well. Figures 4.18 and 4.19 presents a comparison between the control and medium normalized peak area averages of the lipids that decreased significantly. Many of these lipid species are TGs with a few LPCs and 1 DG. Additionally, all lipid species that experienced a statistically significant normalized area decrease after a high dose were present in the medium dose.

A comparison between the low dose livers and the control livers revealed significantly different results compared to the former two analyses. Investigation via Manhattan plot (Figure 4.20) revealed TGs as the only class to exhibit statistically significant changes. Furthermore, analysis via volcano plot (Figure 4.21) illustrated that all have decreased. Overall, statistically significant changes observed are summarized in Table 4.7. Unlike the previous two comparisons, no lipid species were reported to have experienced a statistically significant increase post-low dose radiation exposure. However, lipid species that experienced a significant decrease (Figures 4.22 and 4.23 ) were all TGs and, not all lipids that significantly decreased in the medium dose were present in the low dose. 


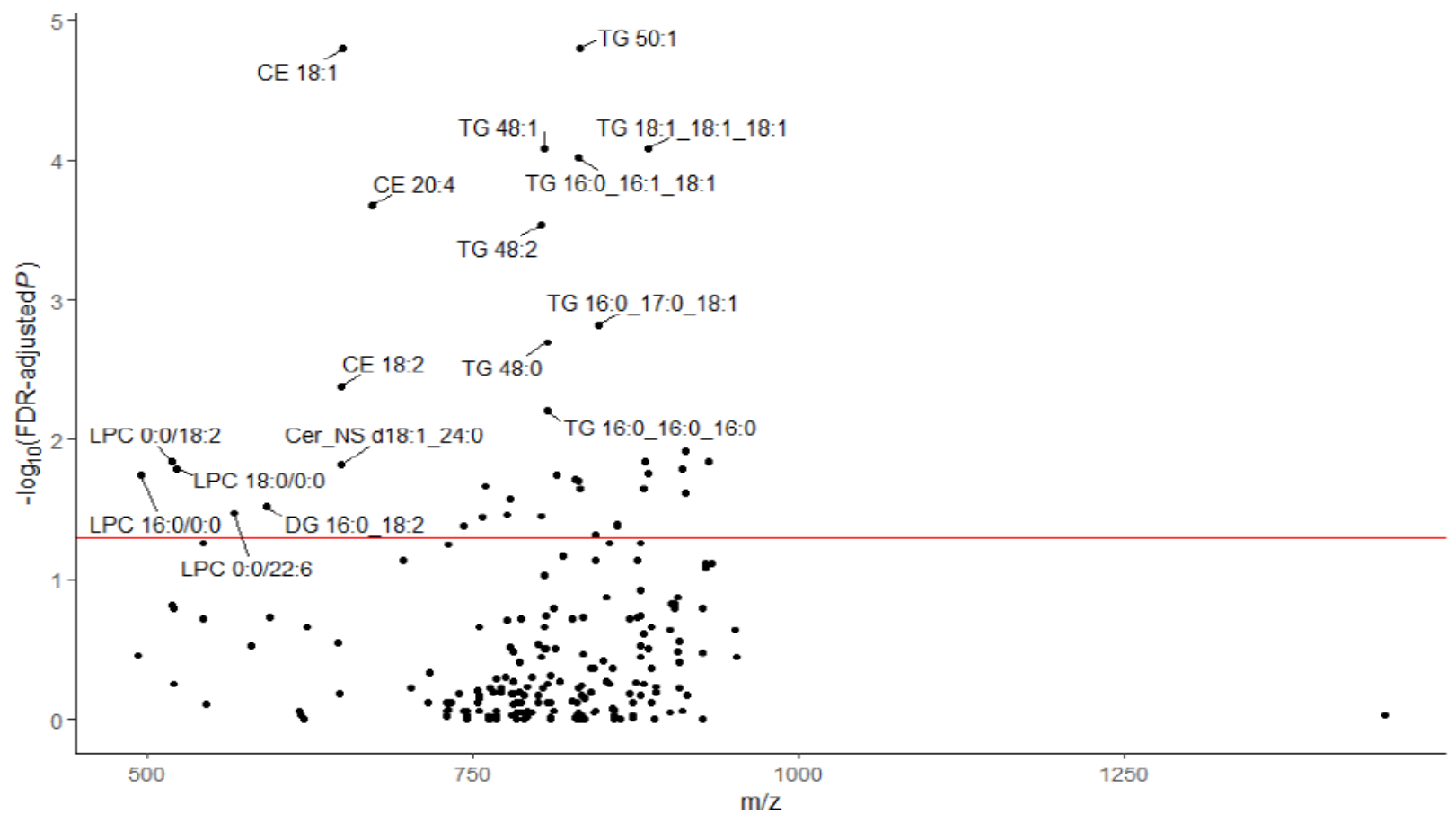

Figure 4.14: Manhattan plot depicting fold change between the medium versus control. Red line indicates the statistically significant line after applying the $\mathrm{BH}$ method. X-axis indicates $\mathrm{m} / \mathrm{z}$.

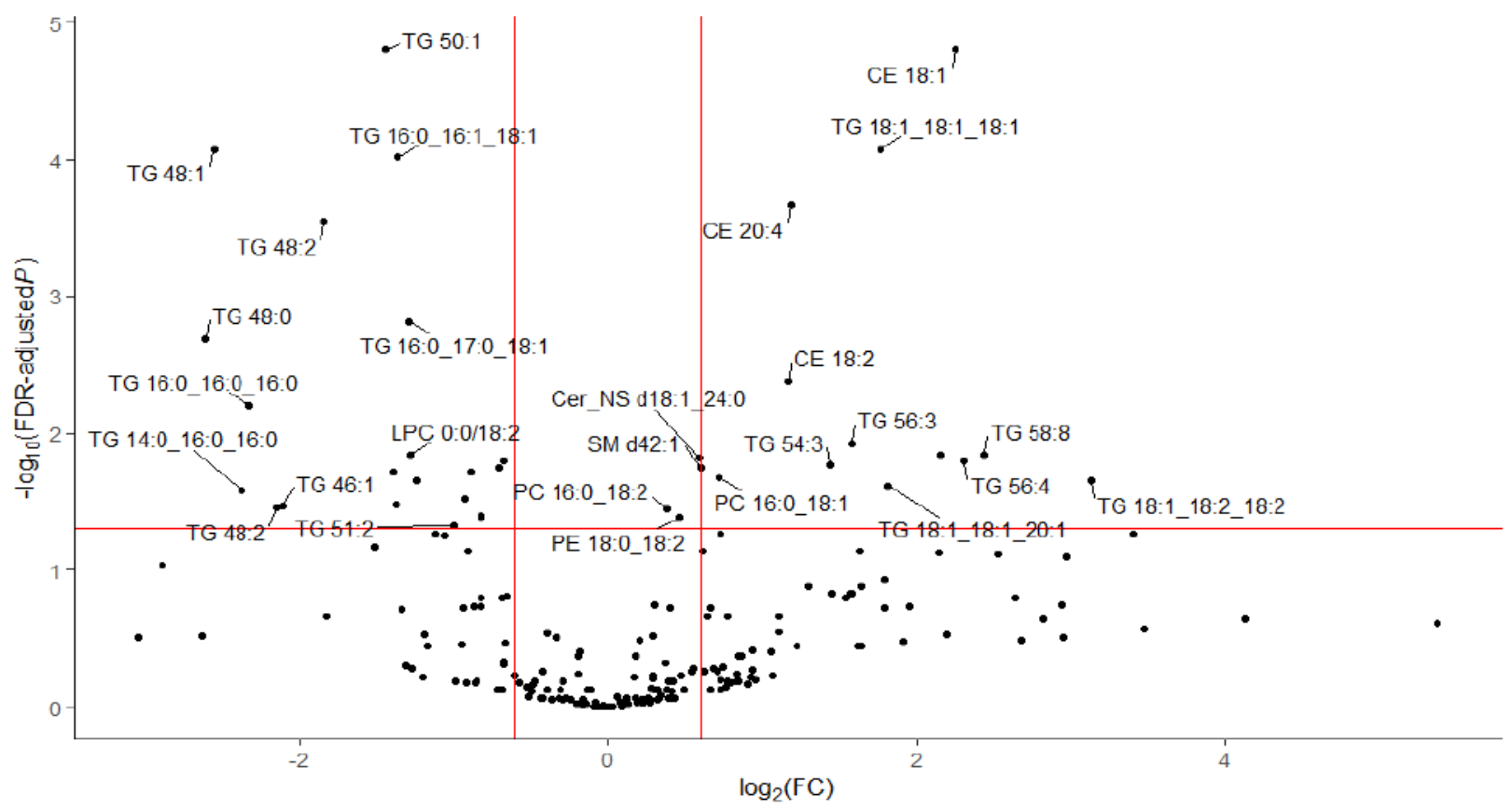

Figure 4.15: Volcano plot depicting fold change between the medium versus control. Red horizontal line indicates the statistically significant line after applying the $\mathrm{BH}$ method. Red vertical lines indicate $\log _{10}(F C)= \pm 0.5$. 
Table 4.5: Lipids showing significant increase in fold change between the medium dosed livers versus control livers.

\begin{tabular}{cccccc}
\hline Compound Name & p_val & FC & Avg. $\mathrm{m} / \mathrm{z}$ & fdr.adj.p_val & ex.thres \\
\hline CE 18:2 & 0.00019458 & 2.24423834 & 648.58528 & 0.00416395 & TRUE \\
CE 18:1 & $1.47 \mathrm{E}-07$ & 4.73997066 & 650.60092 & $1.57 \mathrm{E}-05$ & TRUE \\
CE 20:4 & $6.01 \mathrm{E}-06$ & 2.26730458 & 672.58536 & 0.00021436 & TRUE \\
Cer_NS d18:1_24:0 & 0.00113492 & 1.50268352 & 649.63818 & 0.01517958 & TRUE \\
PE 18:0_18:2 & 0.00690845 & 1.38139091 & 743.54932 & 0.04106687 & TRUE \\
PC 16:0_18:2 & 0.00546791 & 1.30283332 & 757.5639 & 0.03545857 & TRUE \\
PC 16:0_18:1 & 0.00238906 & 1.64933637 & 759.57938 & 0.02130243 & TRUE \\
SM d42:1 & 0.00166833 & 1.51686411 & 814.69356 & 0.01777208 & TRUE \\
TG 18:1_18:2_18:2 & 0.00259982 & 8.73216925 & 880.7526 & 0.02218963 & TRUE \\
TG 18:1_18:1_18:2 & 0.00097582 & 4.42454074 & 882.76845 & 0.01428045 & TRUE \\
TG 18:1_18:1_18:1 & $1.57 E-06$ & 3.3882352 & 884.78428 & $8.40 E-05$ & TRUE \\
TG 54:3 & 0.00152453 & 2.71559442 & 884.784425 & 0.01717104 & TRUE \\
TG 56:4 & 0.00134893 & 4.95588996 & 910.7991 & 0.01603727 & TRUE \\
TG 18:1_18:1_20:1 & 0.00304789 & 3.50921704 & 912.814925 & 0.02415737 & TRUE \\
TG 56:3 & 0.00066508 & 2.98230197 & 912.81502 & 0.01186063 & TRUE \\
TG 58:8 & 0.00100097 & 5.41498847 & 930.767775 & 0.01428045 & TRUE \\
\hline
\end{tabular}

p_value indicated the value from t-test using $\alpha=0.05$, FC indicates the fold change, determined by taking the averaged normalized intensity from the high dose divided by the averaged normalized intensity from the control. Avg. $\mathrm{mz}$ is the average experimental $\mathrm{m} / \mathrm{z}$. fdr.adj.p_value is the $\mathrm{p}$ - value after using the $\mathrm{BH}$ method for false discovery rate. Ex.thres is the output from RStudio indicating whether the lipid species does accrue significant change. True indicating significant change.

Table 4.6: Lipids showing significant decrease in fold change between the medium dosed livers versus control livers.

\begin{tabular}{cccccc}
\hline Compound Name & p_val & FC & Avg. $\mathrm{m} / \mathrm{z}$ & fdr.adj.p_val & ex.thres \\
\hline LPC 16:0/0:0 & 0.00174399 & 0.6152763 & 495.33324 & 0.01777208 & TRUE \\
LPC 0:0/18:2 & 0.00089701 & 0.41265541 & 519.3331 & 0.01428045 & TRUE \\
LPC 18:0/0:0 & 0.00129678 & 0.62854136 & 523.36456 & 0.01603727 & TRUE \\
LPC 0:0/22:6 & 0.00464276 & 0.38586367 & 567.33272 & 0.03311835 & TRUE \\
DG 16:0_18:2 & 0.0040562 & 0.52324346 & 592.50766 & 0.02993196 & TRUE \\
TG 46:1 & 0.00493008 & 0.23166733 & 776.689967 & 0.03403346 & TRUE \\
TG 14:0_16:0_16:0 & 0.00342671 & 0.19371439 & 778.705433 & 0.02618986 & TRUE \\
TG 48:2 & $9.36 E-06$ & 0.27932232 & 802.70584 & 0.00028615 & TRUE \\
TG 48:2 & 0.00521666 & 0.22682664 & 802.705933 & 0.03488642 & TRUE \\
TG 48:1 & $1.20 E-06$ & 0.17041349 & 804.72168 & $8.40 E-05$ & TRUE \\
TG 48:0 & $8.54 E-05$ & 0.16446023 & 806.737 & 0.00203062 & TRUE \\
\hline
\end{tabular}




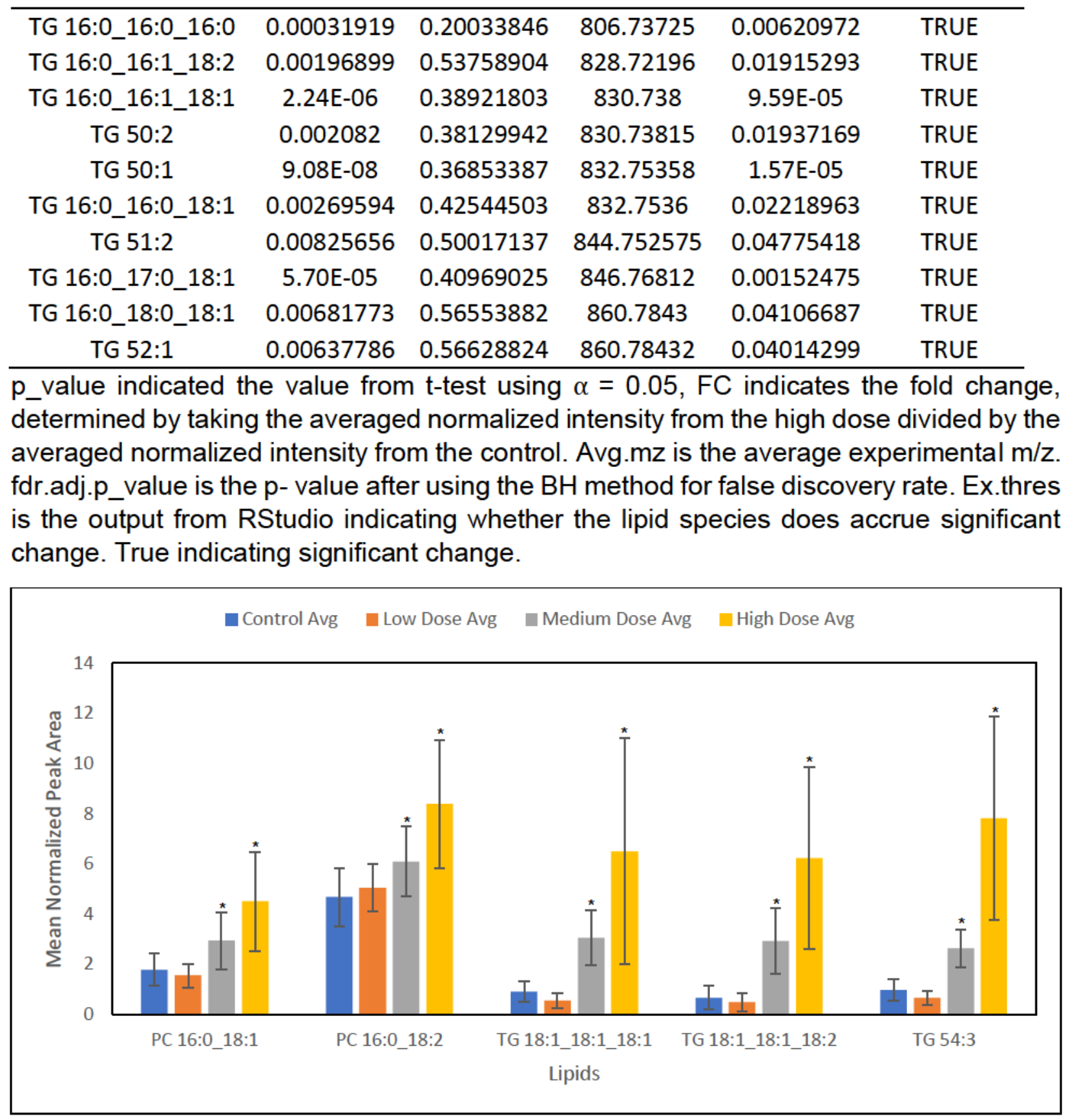

Figure 4.16: Relative abundance of the top 5 lipids with the highest normalized peak area averages in rat liver control (blue) versus medium (grey) dose samples that increased in a statistically significant manner after applying an FDR correction. Low (orange) and high (yellow) dose levels were included to show the changes in those categories. 


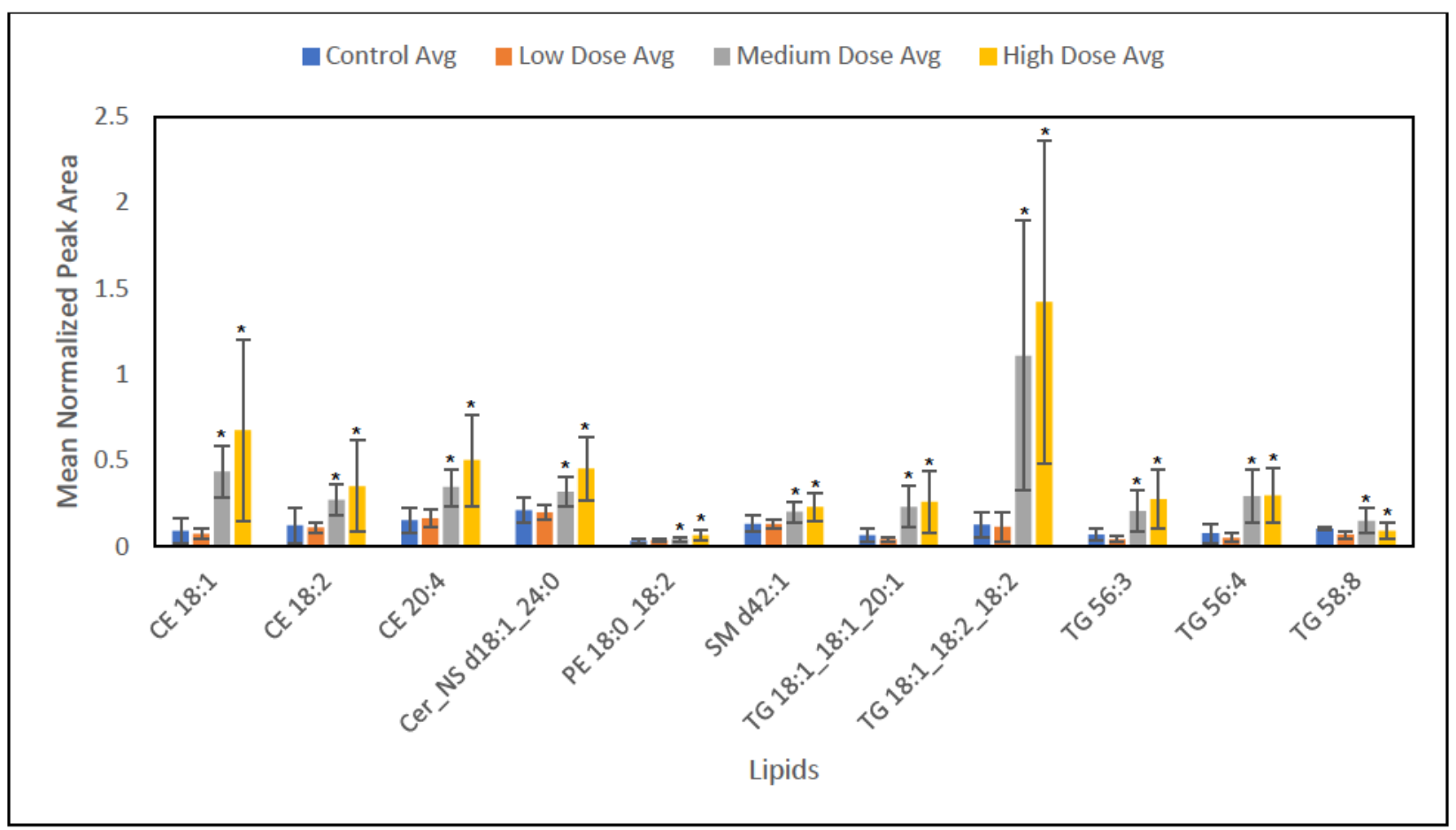

Figure 4.17: Relative abundance of the 11 lipids with normalized peak area averages in rat liver control (blue) versus medium (grey) dose samples that increased in a statistically significant manner after applying an FDR correction. Low (orange) and high (yellow) dose levels were included to show the changes in those categories.

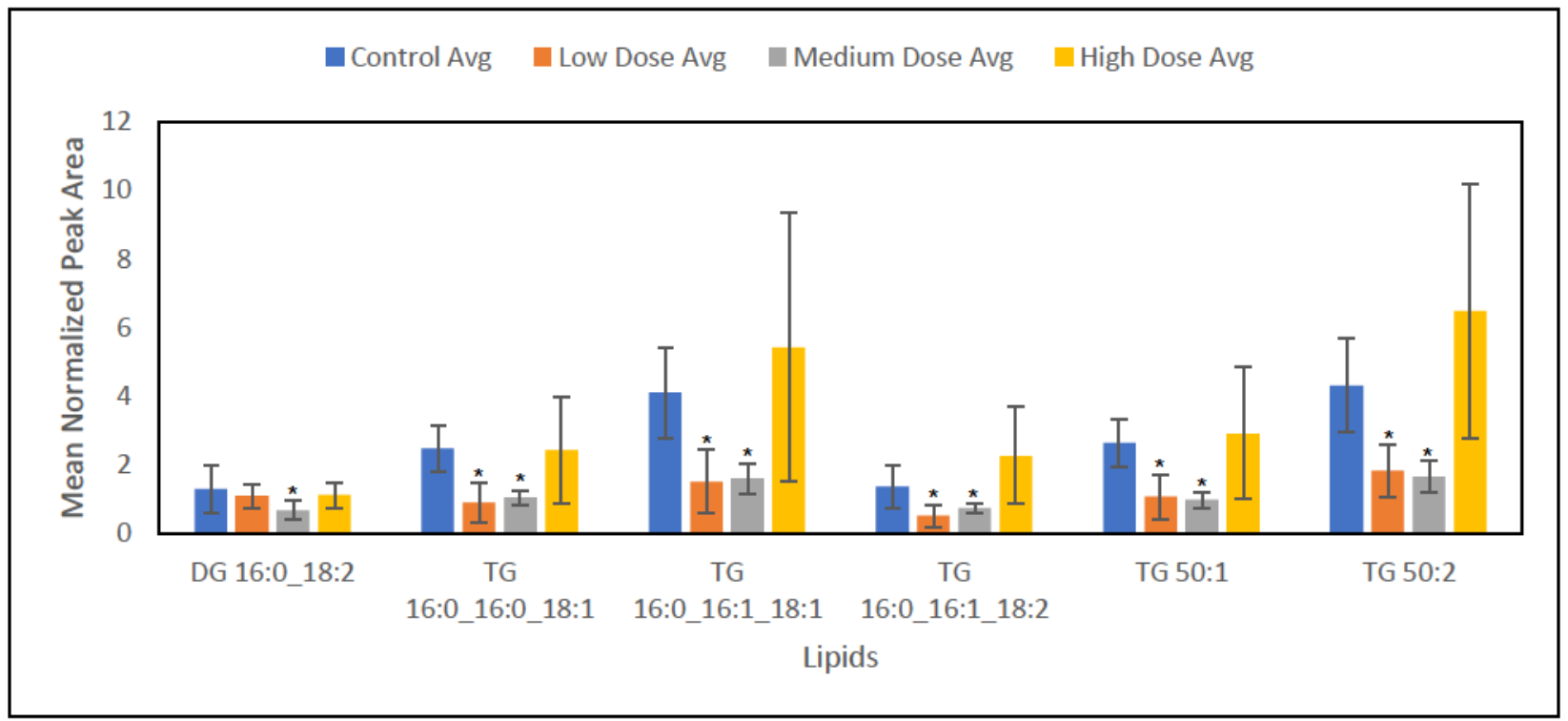

Figure 4.18: Relative abundance of the top 6 lipids with the highest normalized peak area averages in rat liver control (blue) versus medium (grey) dose samples that decreased in a statistically significant manner after applying an FDR correction. Low (orange) and high (yellow) dose levels were included to show the changes in those categories. 


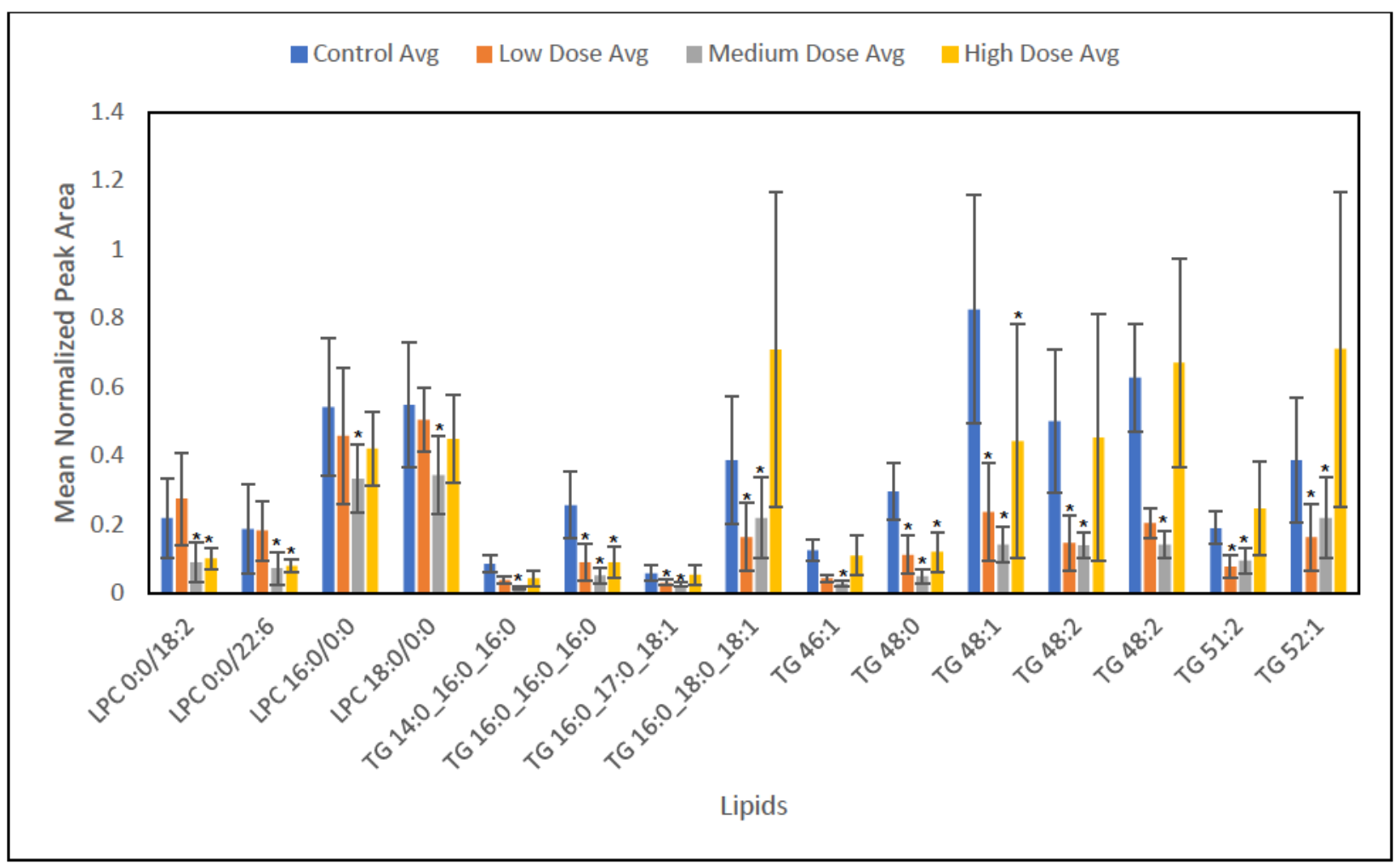

Figure 4.19: Relative abundance of the 15 lipids with normalized peak area averages in rat liver control (blue) versus medium (grey) dose samples that decreased in a statistically significant manner after applying an FDR correction. Low (orange) and high (yellow) dose levels were included to show the changes in those categories. 


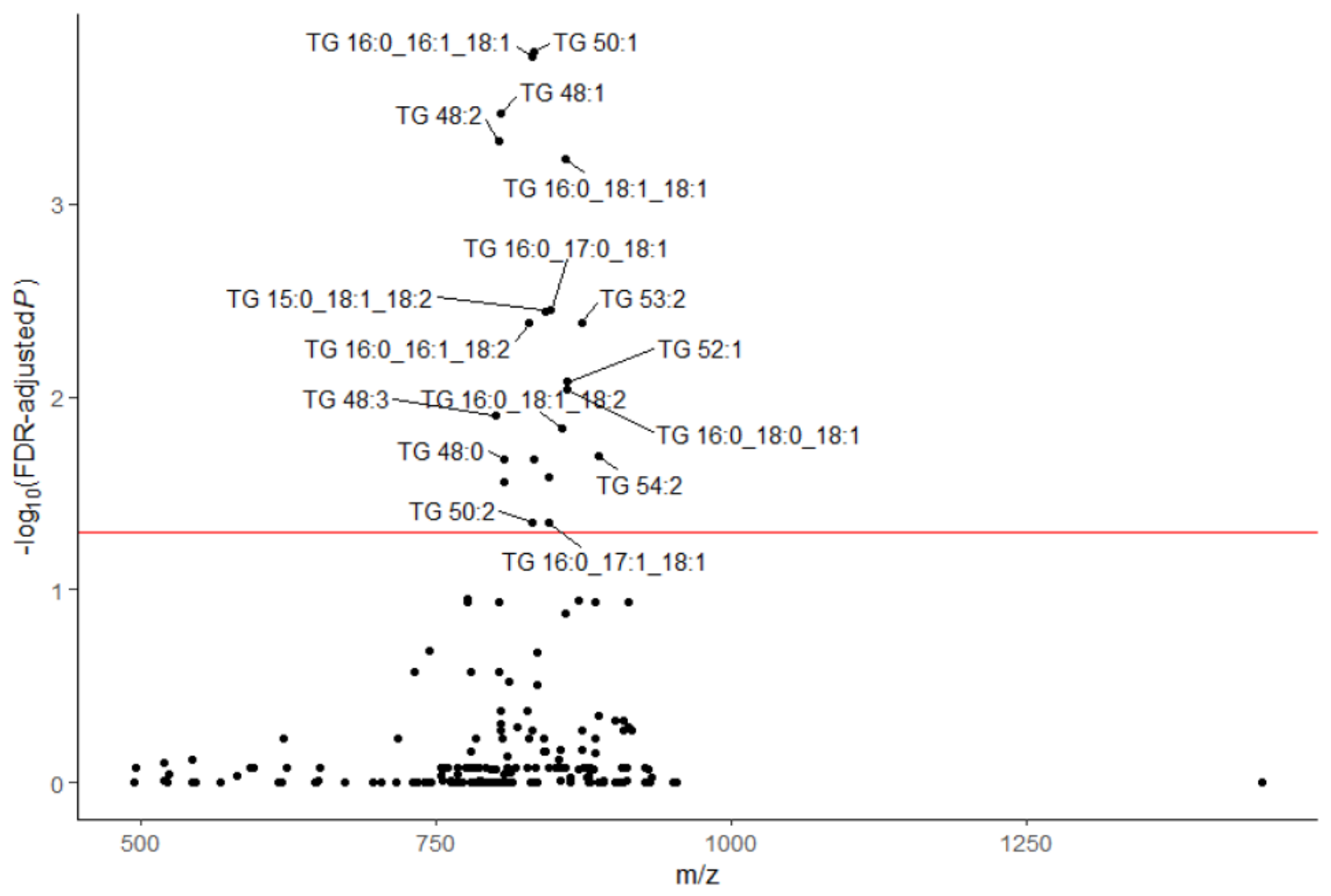

Figure 4.20: Manhattan plot depicting fold change between the low versus control. Red line indicates the statistically significant line after applying the $\mathrm{BH}$ method. X-axis indicates $\mathrm{m} / \mathrm{z}$. 


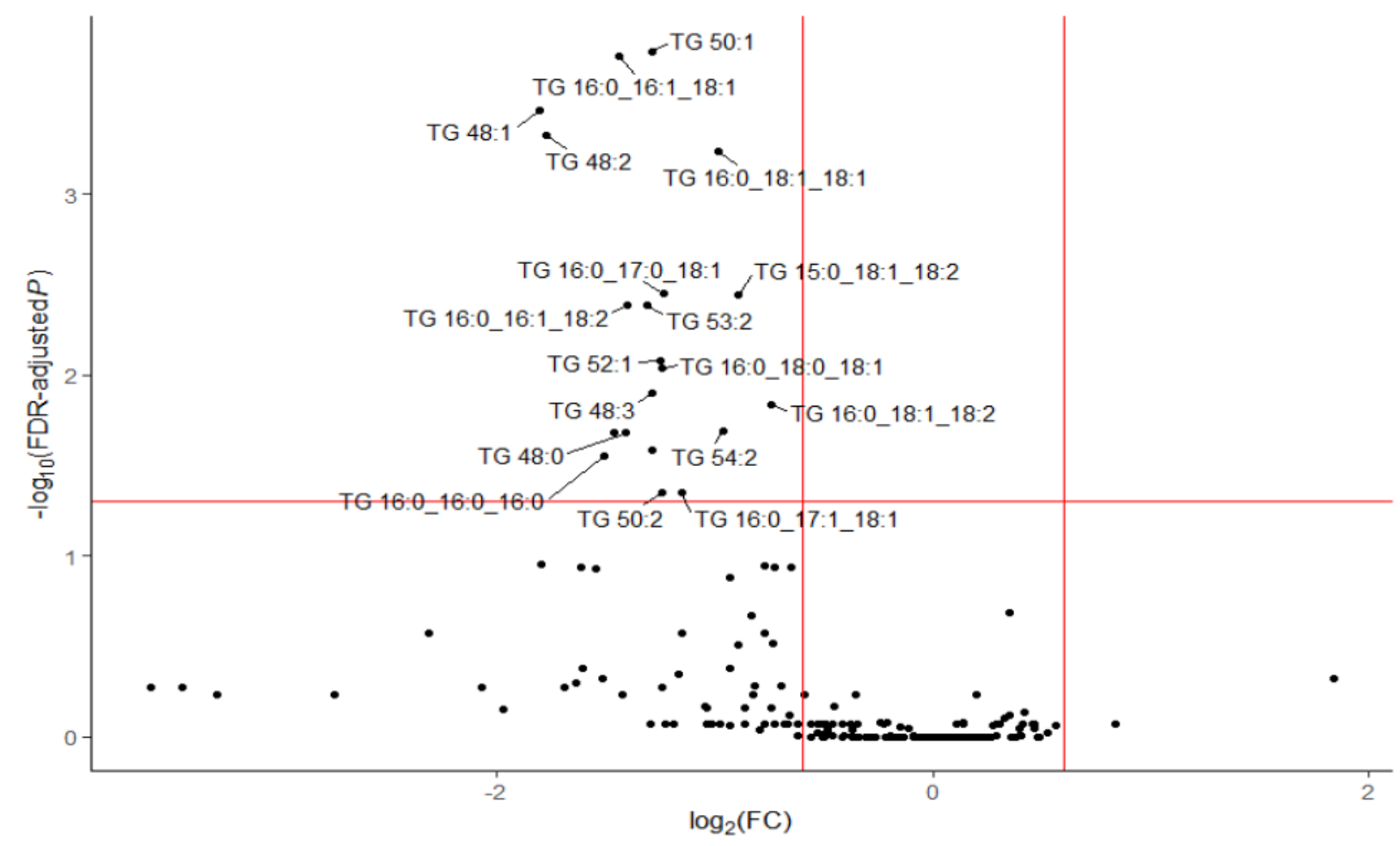

Figure 4.21: Volcano plot depicting fold change between the low versus control. Red horizontal line indicates the statistically significant line after applying the $\mathrm{BH}$ method. Red vertical lines indicate $\log _{10}(F C)= \pm 0.5$.

Table 4.7: Lipids showing significant decrease in fold change between the low dosed livers versus control livers.

\begin{tabular}{cccccc}
\hline Compound Name & p_val & FC & Avg. $\mathrm{m} / \mathrm{z}$ & fdr.adj.p_val & ex.thres \\
\hline TG 48:3 & 0.00069916 & 0.41002625 & 800.68962 & 0.01246826 & TRUE \\
TG 48:2 & $8.84 \mathrm{E}-06$ & 0.29204282 & 802.70584 & 0.00047294 & TRUE \\
TG 48:1 & $4.79 E-06$ & 0.28621518 & 804.72168 & 0.00034169 & TRUE \\
TG 48:0 & 0.00156641 & 0.37644591 & 806.737 & 0.02095072 & TRUE \\
TG 16:0_16:0_16:0 & 0.00234496 & 0.35195165 & 806.73725 & 0.02787902 & TRUE \\
TG 16:0_16:1_18:2 & 0.0001726 & 0.37843941 & 828.72196 & 0.00410414 & TRUE \\
TG 16:0_16:1_18:1 & $1.61 E-06$ & 0.36807393 & 830.738 & 0.00017227 & TRUE \\
TG 50:2 & 0.00408792 & 0.4228471 & 830.73815 & 0.04465424 & TRUE \\
TG 50:1 & $7.56 E-07$ & 0.40890836 & 832.75358 & 0.00016178 & TRUE \\
TG 16:0_16:0_18:1 & 0.00150212 & 0.36283833 & 832.7536 & 0.02095072 & TRUE \\
TG 15:0_18:1_18:2 & 0.00011739 & 0.53900651 & 842.73696 & 0.0035889 & TRUE \\
TG 51:2 & 0.00205731 & 0.41003661 & 844.752575 & 0.0258979 & TRUE \\
TG 16:0_17:1_18:1 & 0.00417329 & 0.44895885 & 844.752875 & 0.04465424 & TRUE \\
TG 16:0_17:0_18:1 & $9.96 E-05$ & 0.42356672 & 846.76812 & 0.0035524 & TRUE \\
TG 16:0_18:1_18:2 & 0.00089285 & 0.59766293 & 856.75412 & 0.0146976 & TRUE \\
\hline
\end{tabular}




\begin{tabular}{cccccc}
\hline TG 16:0_18:1_18:1 & $1.36 \mathrm{E}-05$ & 0.503704 & 858.7697 & 0.00058208 & TRUE \\
TG 16:0_18:0_18:1 & 0.00047162 & 0.42187123 & 860.7843 & 0.00917506 & TRUE \\
TG 52:1 & 0.00039072 & 0.41898976 & 860.78432 & 0.00836143 & TRUE \\
TG 53:2 & 0.00016548 & 0.40289232 & 872.7838 & 0.00410414 & TRUE \\
TG 54:2 & 0.00132579 & 0.51265833 & 886.7998 & 0.02026568 & TRUE \\
\hline
\end{tabular}

p_value indicated the value from t-test using $\alpha=0.05$, FC indicates the fold change, determined by taking the averaged normalized intensity from the high dose divided by the averaged normalized intensity from the control. Avg.mz is the average experimental m/z. fdr.adj.p_value is the $\mathrm{p}$ - value after using the $\mathrm{BH}$ method for false discovery rate. Ex.thres is the output from RStudio indicating whether the lipid species does accrue significant change. True indicating significant change.

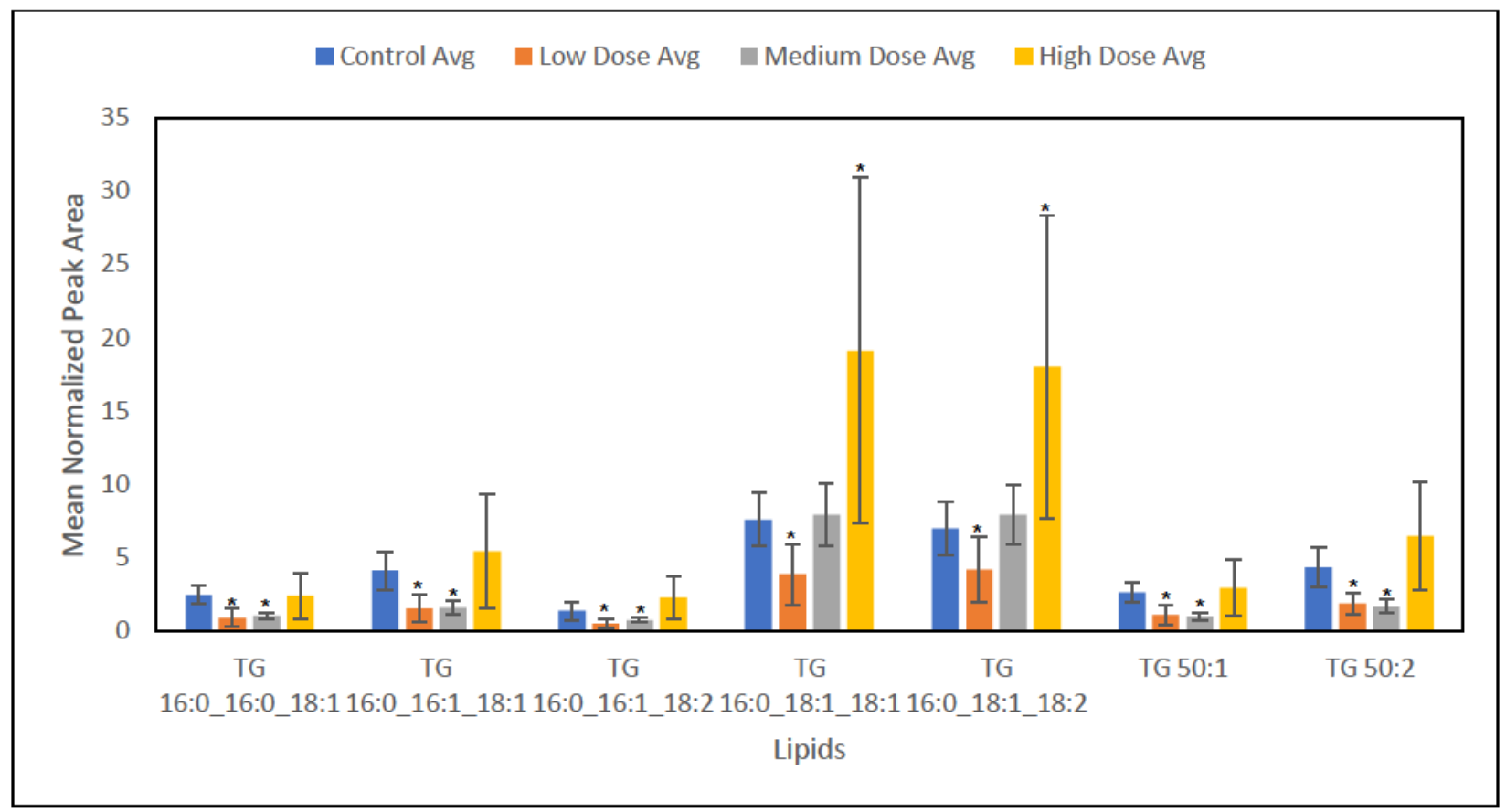

Figure 4.22: Relative abundance of the top 7 lipids with the highest normalized peak area averages in rat liver control (blue) versus low (orange) dose samples that decreased in a statistically significant manner after applying an FDR correction. Medium (grey) and high (yellow) dose levels were included to show the changes in those categories. 


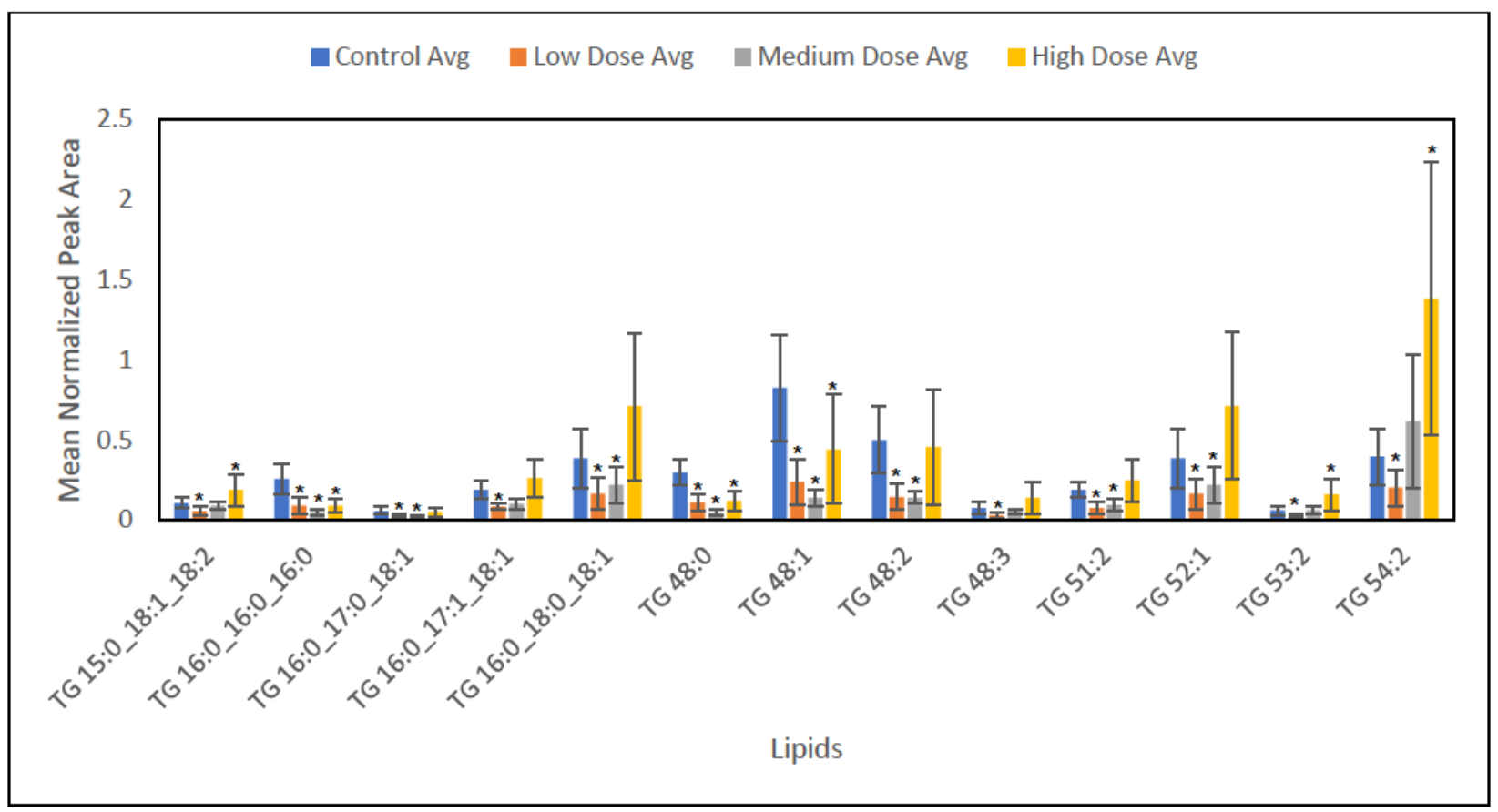

Figure 4.23: Relative abundance of the 13 lipids with normalized peak area averages in rat liver control (blue) versus low (orange) dose samples that decreased in a statistically significant manner after applying an FDR correction. Medium (grey) and high (yellow) dose levels were included to show the changes in those categories.

In this study, we report lipidomic changes that occur because of LDR exposure. Perturbation of lipid metabolism due to LDR is evident based on the results obtained in this chapter. The animal was sacrificed 10 days post initial radiation exposure to allow changes to occur. It is noteworthy that discussions surrounding lipid metabolism are highly dependent on both untargeted and targeted literature studies to provide valuable insight to possible explanations of the results collected above since conducting these experiments is beyond the scope of this thesis. In literature, it is reported that polyunsaturated TGs $60: 10,60: 11$, and 62:14 significantly increased in serum after 10 Gy of radiation. ${ }^{4}$ These lipids primarily contained acyl chains $20: 4$ and 22:6. Both acyl moieties 20:4 (arachidonic acid) and 22:6 (docosahexaenoic acid) are involved in the cyclooxygenase (COX) and lipoxygenase (LOX) pathway, signalling inflammation. ${ }^{2}$ While none of the TGs in Tables 4.3 and 4.5 and Figures 4.11-4.12 and 4.16-4.17 are 60:10, 
60:11, or 62:14, many TGs listed (at least the ones with a defined dominant constituent) do contain acyl moieties $20: 4$ or $22: 6$. It is theorized that these TGs are synthesized at an increased rate to serve as a reservoir for possible transport to tissues. ${ }^{4}$ This aligns with what was observed in this study since three polyunsaturated TGs (TG 56:8, $58: 9$ and 56:7) all contain a 22:6 acyl moiety. These three lipids are observed to have experienced a statistically significant increase in the high-dose rat livers.

While we did not report any esterified form of 20:4 and 22:6 acyls in higher dosage, free fatty acid (FFA) 20:4 (arachidonic acid), an essential polyunsaturated fatty acid derived from free fatty acid 18:2 (linoleic acid), is typically esterified for storage. Arachidonic acid is a product from the cleavage of phospholipids by phospholipase $A 2^{18}$ which many inflammatory molecules are produced from. ${ }^{4}$ Additionally, FFA 22:6 (docosahexaenoic acid) is derived from FFA 22:5 and it inhibits inflammation, tumour growth and apoptosis. ${ }^{4}$ Increase esterification of these FFA suggests upregulation for possible transport to tissues to synthesize into TGs and $\mathrm{GPs}^{4}$, which may explain the statistically significant increase of PC and TGs containing 20:4 and 22:6 acyl moieties in high dose livers. While this theory may rationalize a small percentage of the results observed, further investigations must be conducted to further cement this hypothesis with the results presented.

Many of the significantly increased TGs observed post LDR exposure contain acyl moieties 18:1 (oleic acid), 18:2 (linoleic acid) and, 16:0 (palmitic acid). It is postulated that the synthesis of TGs from these acyl moieties may be an indirect result of radiation exposure. In serum, Laiakis et al. reported that TG lipids exhibited a statistically significant decrease in respect to their non-radiated samples. In addition, they observe an increase 
in MG 18:1, MG 18:2, TG 48:0, and TG 50:3 in circulation while other lipids exhibit decreases in circulation contrast to no radiation exposure. ${ }^{19}$ In Table 4.4, 4.6, and 4.7, a significant decrease of TG 48:0 is observed across all three dosage levels in the liver and a significant decrease of TG 50:3 (TG 16:0_16:1_18:2) is observed across the low and medium dosage levels in the liver. Although it is speculated that synthesis of TGs as a result of increase MG 18:1 and MG 18:2 in circulation, in literature, it is contradicted as this may be attributed to nutritional intake. ${ }^{2,19}$ The idea of TG synthesis following radiation exposure was once reported by Wooles who discovered an increased concentration of liver TGs following irradiation. In the study, Wooles demonstrated that marked palmitic acid (with ${ }^{14} \mathrm{C}$ ) was converted into TG in the liver at different time points post-radiation exposure. ${ }^{20}$ Further investigations are required to determine whether metabolically Wooles', Pannkuk or Laiakis' studies align with the results presented in this thesis. Pannkuk et al. demonstrated that the concentration of LPCs, particularly LPC 0:0/18:2 and 0:0/22:6 have decreased significantly which contradicts literature. As mentioned, typically the concentration of LPC increases as a result of cleavage of phospholipids by phospholipase A2. Pannkuk et al. demonstrated that many LPCs (from 16:0 to 22:6) primarily had the highest mean in the high dose. Although Pannkuk et al. studied using non-human primates' serum, it was hypothesized that similar results would be observed in liver tissues. Potentially, other factors may contribute to a decrease of LPC with increasing radiation dose however, further investigation is beyond the scope of this work. LDR may also affect species of CE. As observed in Figures 4.12 and 4.17, a significant increase of SMs and CEs were reported with increasing radiation dose. Temporal experiments were conducted in literature which reports an insignificant increase in CEs 
and SMs after 10 days (same time Wistar rats in this study were kept alive) ${ }^{2}$ Although a hypothesis was not provided, SMs are extensively studied in radiation exposure studies since they can undergo free radical fragmentation, forming ceramides that are involved in apoptosis. ${ }^{21}$ We also present in this study, a significant increase in Cer_NS d18:1_24:0 following LDR exposure which possibly indicates cell stress.

Mass spectrometry studies have served to provide insight into lipidomic changes because of radiation exposure. The untargeted approach has allowed the analysis of many lipid classes and species. Significant changes in the lipidome may provide insight into overall metabolism. This most common method is to analyze various analytes that indicate cell stress, lipid peroxidation, and damage. However, this requires additional investigations and while some hypotheses were provided based on literature, conducting these studies is beyond the scope of this thesis. However, based on the data obtained in this chapter, we report significant changes in the liver lipidome when exposed to LDR.

\subsection{Conclusion}

It has been found that LDR exposure has drastic changes in the overall liver lipidome when treated with three different concentrations of ${ }^{210} \mathrm{Po}$ solutions. Compared to the non-radiated controls, many of the lipids, particularly TGs, exhibit significantly higher levels because of LDR exposure. This may be due to the increased synthesis of TGs at an increased rate to serve as a reservoir for possible transport to tissues. While not analyzed directly, few TGs identified contain acyl moieties related to the LOX/COX pathway, signalling inflammation. In addition, it is postulated that while many TGs consist of acyl moieties like $18: 1$ and 18:2 that derive into acyl moieties that trigger an 
inflammatory response. While much of the explanation is solely based on literature,

further analysis is recommended to determine whether these hypotheses remain true.

\subsection{References}

(1) Shin, E.; Lee, S.; Kang, H.; Kim, J.; Kim, K.; Youn, H.; Jin, Y. W.; Seo, S.; Youn, B. Organ-Specific Effects of Low Dose Radiation Exposure: A Comprehensive Review. Front. Genet. 2020, 11, 1-11. https://doi.org/10.3389/fgene.2020.566244.

(2) Pannkuk, E. L.; Laiakis, E. C.; Singh, V. K.; Fornace, A. J. Lipidomic Signatures of Nonhuman Primates with Radiation-Induced Hematopoietic Syndrome. Sci. Rep. 2017, 7 (9777), 9. https://doi.org/10.1038/s41598-017-10299-w.

(3) Azab, K.; Bashandy, M.; Salem, M.; Ahmed, O.; Tawfik, Z.; Helal, H. Royal Jelly Modulates Oxidative Stress and Tissue Injury in Gamma Irradiated Male Wister Albino Rats. North Am. J. Med. Sci. 2011, 3 (6), 268-276. https://doi.org/10.4297/najms.2011.3268.

(4) Pannkuk, E. L.; Laiakis, E. C.; Mak, T. D.; Astarita, G.; Authier, S.; Wong, K.; Fornace, A. J. A Lipidomic and Metabolomic Serum Signature from Nonhuman Primates Exposed to Ionizing Radiation. Metabolomics 2016, 12 (80), 11. https://doi.org/10.1007/s11306-016$1010-0$.

(5) Li, L.; Zhong, S.; Shen, X.; Li, Q.; Xu, W.; Tao, Y.; Yin, H. Recent Development on Liquid Chromatography-Mass Spectrometry Analysis of Oxidized Lipids. Free Radic. Biol. Med. 2019, 144, 16-34. https://doi.org/10.1016/j.freeradbiomed.2019.06.006.

(6) Maqsudur Rashid, A.; Ramalingam, L.; Al-Jawadi, A.; Moustaid-Moussa, N.; Moussa, H. Low Dose Radiation, Inflammation, Cancer and Chemoprevention. Int. J. Radiat. Biol. 2019, 95 (4), 506-515. https://doi.org/10.1080/09553002.2018.1484194.

(7) Wang, Y.; Bannister, L. A.; Sebastian, S.; Le, Y.; Ismail, Y.; Didychuk, C.; Richardson, R. B.; Flegal, F.; Paterson, L. C.; Causey, P.; Fawaz, A.; Wyatt, H.; Priest, N.; Klokov, D. Low-Dose Radiobiology Program at Canadian Nuclear Laboratories: Past, Present, and Future. Int. J. Radiat. Biol. 2019, 95 (10), 1361-1371. https://doi.org/10.1080/09553002.2018.1562252.

(8) Tapio, S.; Little, M. P.; Kaiser, J. C.; Impens, N.; Hamada, N.; Georgakilas, A. G.; Simar, D.; Salomaa, S. Ionizing Radiation-Induced Circulatory and Metabolic Diseases. Environ. Int. 2021, 146 (106235), 16. https://doi.org/10.1016/j.envint.2020.106235.

(9) Jr, J. D. B.; Clarke, R. H.; Meinhold, C. B.; Sasaki, Y.; Shandala, N.; Jr, F. A. M.; Sinclair, W. K.; Streffer, C.; Pan, Z.; Sugier, A.; Pentreath, R. J.; Preston, R. J. Members of the Main Commission of the ICRP. 328. 
(10) Little, M. P.; Wakeford, R.; Tawn, E. J.; Bouffler, S. D.; Berrington de Gonzalez, A. Risks Associated with Low Doses and Low Dose Rates of Ionizing Radiation: Why Linearity May Be (Almost) the Best We Can Do. Radiology 2009, 251 (1), 6-12. https://doi.org/10.1148/radiol.2511081686.

(11) Introduction to Radiation; Canadian Nuclear Safety Commission, Ed.; Canadian Nuclear Safety Commission: Ottawa, 2012.

(12) Pannkuk, E. L.; Laiakis, E. C.; Authier, S.; Wong, K.; Fornace, A. J. Targeted Metabolomics of Nonhuman Primate Serum after Exposure to Ionizing Radiation: Potential Tools for High-Throughput Biodosimetry. RSC Adv. 2016, 6, 51192-51202. https://doi.org/10.1039/C6RA07757A.

(13) Goudarzi, M.; Weber, W.; Mak, T. D.; Chung, J.; Doyle-Eisele, M.; Melo, D.; Brenner, D. J.; Guilmette, R. A.; Fornace, A. J. Development of Urinary Biomarkers for Internal Exposure by Cesium-137 Using a Metabolomics Approach in Mice. Radiat. Res. 2013, 181 (1), 54. https://doi.org/10.1667/RR13479.1.

(14) Goudarzi, M.; Weber, W. M.; Mak, T. D.; Chung, J.; Doyle-Eisele, M.; Melo, D. R.; Brenner, D. J.; Guilmette, R. A.; Fornace, A. J. Metabolomic and Lipidomic Analysis of Serum from Mice Exposed to an Internal Emitter, Cesium-137, Using a Shotgun LC-MS E Approach. J. Proteome Res. 2015, 14 (1), 374-384. https://doi.org/10.1021/pr500913n.

(15) Seiler, R. L.; Wiemels, J. L. Occurrence of 210 Po and Biological Effects of Low-Level Exposure: The Need for Research. Environ. Health Perspect. 2012, 120 (9), 1230-1237. https://doi.org/10.1289/ehp.1104607.

(16) Drobnik, W.; Liebisch, G.; Audebert, F.-X.; Fröhlich, D.; Glück, T.; Vogel, P.; Rothe, G.; Schmitz, G. Plasma Ceramide and Lysophosphatidylcholine Inversely Correlate with Mortality in Sepsis Patients. J. Lipid Res. 2003, 44, 754-761. https://doi.org/10.1194/jlr.M200401-JLR200.

(17) Whitehead, S. N.; Hou, W.; Ethier, M.; Smith, J. C.; Bourgeois, A.; Denis, R.; Bennett, S. A. L.; Figeys, D. Identification and Quantitation of Changes in the Platelet Activating Factor Family of Glycerophospholipids over the Course of Neuronal Differentiation by High-Performance Liquid Chromatography Electrospray Ionization Tandem Mass Spectrometry. Anal. Chem. 2007, 79 (22), 8539-8548. https://doi.org/10.1021/ac0712291.

(18) Calder, P. C. Polyunsaturated Fatty Acids, Inflammation, and Inflammatory Diseases. In The psychoneuroimmunology of chronic disease: Exploring the links between inflammation, stress, and illness.; Kendall-Tackett, K., Ed.; American Psychological Association: Washington, 2010; pp 77-109. https://doi.org/10.1037/12065-004.

(19) Laiakis, E. C.; Pannkuk, E. L.; Chauthe, S. K.; Wang, Y.-W.; Lian, M.; Mak, T. D.; Barker, C. A.; Astarita, G.; Fornace, A. J. A Serum Small Molecule Biosignature of Radiation Exposure from Total Body Irradiated Patients. J. Proteome Res. 2017, 16 (10), 23. 
(20) Wooles, W. R. Triglyceride Synthesis Following Whole-Body X-Irradiation in the Rabbit. Radiat. Res. 1967, 30 (4), 788. https://doi.org/10.2307/3572144.

(21) Kolesnick, R.; Fuks, Z. Radiation and Ceramide-Induced Apoptosis. Oncogene 2003, 22 (37), 5897-5906. https://doi.org/10.1038/sj.onc.1206702. 


\section{Chapter 5: Conclusion and Future Works}

\subsection{Conclusion}

The use of liquid chromatography coupled with a high-resolution mass spectrometer was demonstrated to be a powerful analytical tool applicable for studying the effects of low dose radiation exposure in an untargeted lipidomics study. To study the effects accurately, optimization was conducted to improve the accuracy and coverage of lipids in high and low abundance. The development of a lipidomics workflow involving iterative acquisition successfully identified less abundant lipids, while investigations of different mobile phase gradients led to one that provided better coverage of TGs. Future work in repeating the optimizing experiments for the analysis of lipids in negative ion mode is recommended for better lipidomic representation.

The effects of LDR exposure on a liver lipidome were performed where the optimized LCMS method was applied. Analysis of four different dosage categories (control, low, medium and, high) of rat liver resulted in dynamic changes in the lipidome. Many of the significant changes occurred in the TG class. Although metabolic studies were not conducted, as it is beyond the focus of this thesis, literature findings assisted in providing hypotheses to the results collected. While further investigations are recommended, see Chapter 5.2, the information collected in this thesis will provide proof that LDR exposure does result in lipidomic changes in the liver. 


\section{$5.2 \quad$ Future Works}

The lipids studied in this project do not represent the entire liver lipidome. Many other classes of lipids (ex. PI, PS, PA, and PG) are typically analyzed using negative ion mode which is beyond the scope of this thesis work. While many major classes were covered, other lipid classes like MGs or free fatty acids are potentially critical in providing insight into the effects of lipid metabolism when exposed to low doses of radiation. All the Wistar rats used in this study were male which may lead to potential biases as female rats may react differently when exposed to radiation. Investigating sex-based differences may be an avenue since literature reports female non-human primates showed greater decrease of PCs in serum post irradiation. While Agilent's Lipid Annotator was able to fully identify many lipid species, many of the features illustrated were not identified which

may present errors as some may be lipids. As the in-silico library grows, potential lipid classes/species may be fully identified, providing greater insight to the liver lipidome. The potential for further analysis of these non-fully identified features could be studied in the future. Furthermore, while the liver is a vital organ, additional analysis on other organs such as the kidneys, heart, muscle, lungs, skin, or brain may provide further insight into the effects of LDR exposure. ${ }^{210} \mathrm{Po}$ is mainly distributed in soft tissues like the spleen, liver, and kidneys. In humans, the liver and the kidneys are primary organs since excretion of heavy metals are transported through blood and shuttled through these two organs. Other organs such as the heart, muscle, lungs, skin, and the brain are based on curiosity and whether LDR influences them. 


\section{Appendix: Supplementary Information}

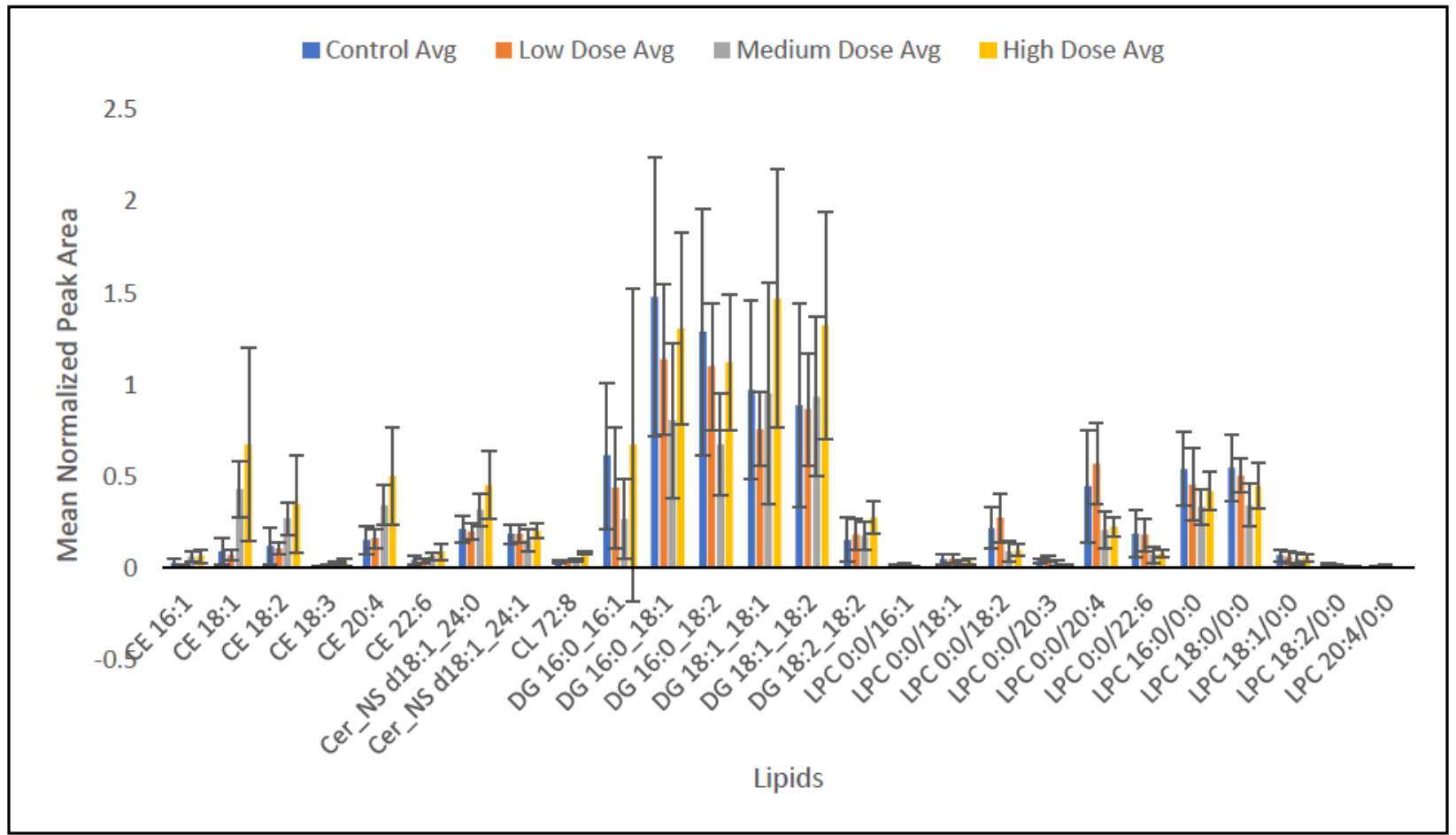

Figure S1: Histogram plot of mean normalized intensity lipids that were acquired using the 6546 QTOF. Samples were analyzed using Agilent's Profinder and RStudio.

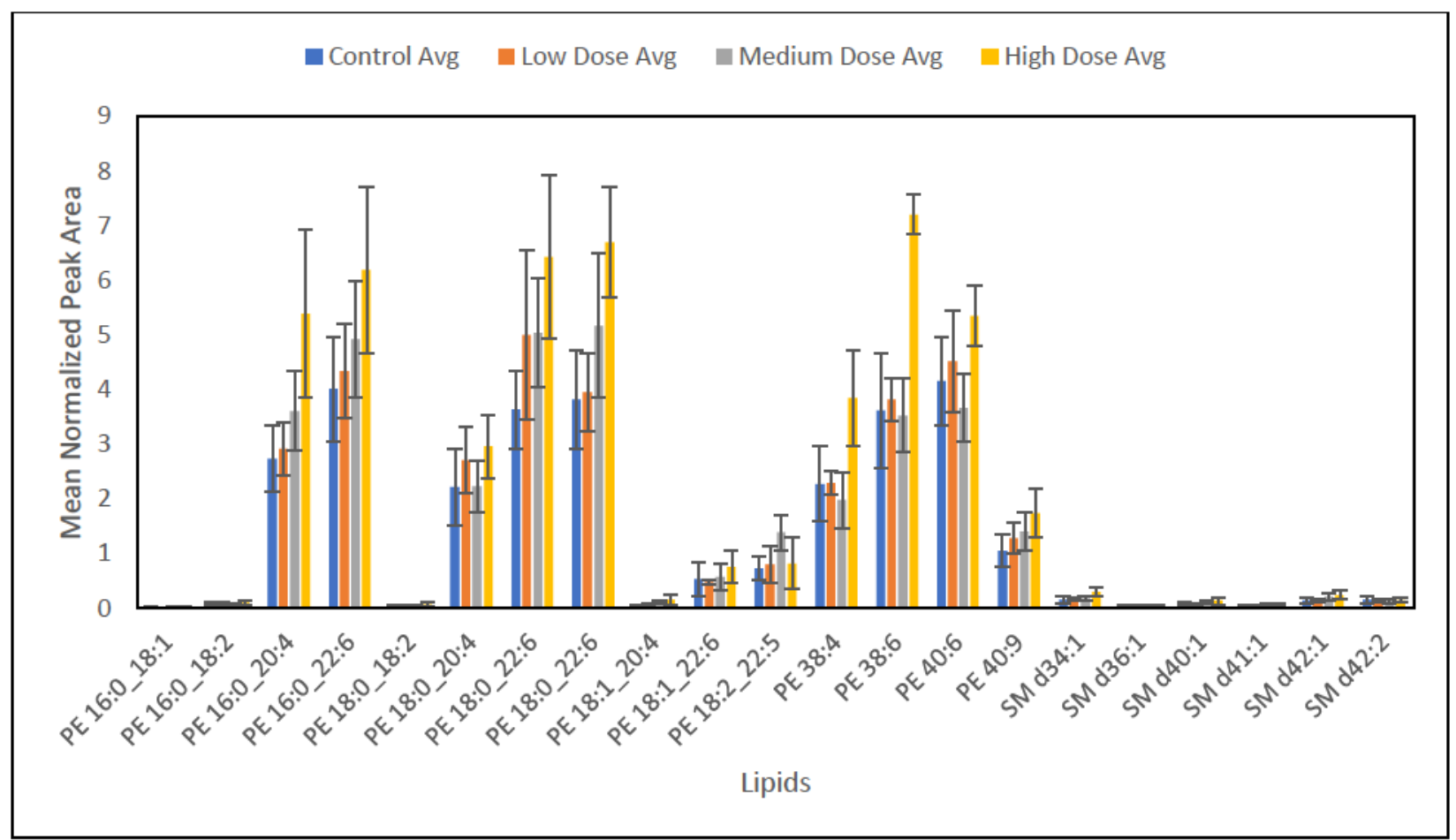

Figure S2: Histogram plot of mean normalized intensity lipids that were acquired using the 6546 QTOF. Samples were analyzed using Agilent's Profinder and RStudio. 


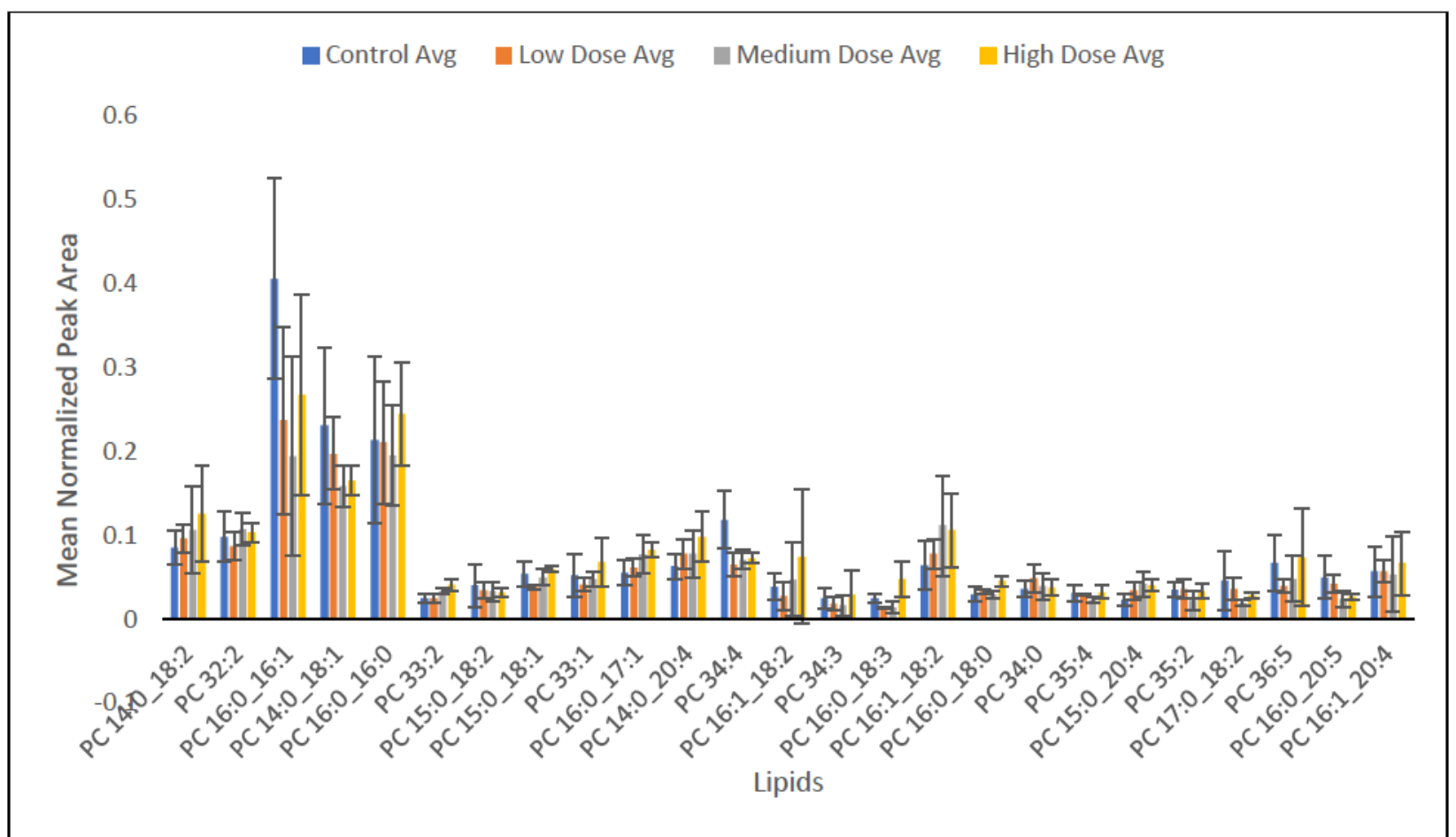

Figure S3: Histogram plot of mean normalized intensity lipids that were acquired using the 6546 QTOF. Samples were analyzed using Agilent's Profinder and RStudio.

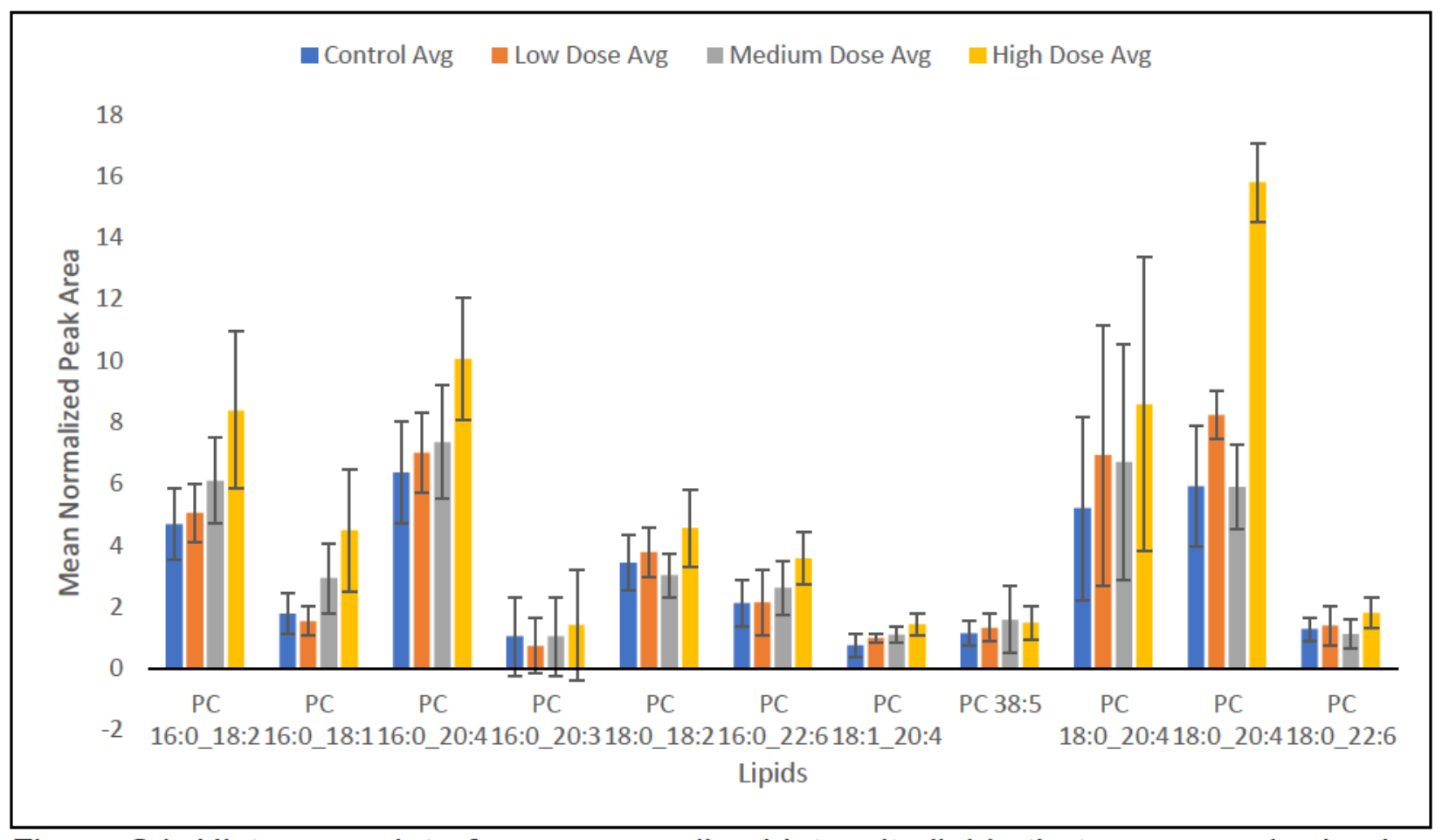

Figure S4: Histogram plot of mean normalized intensity lipids that were acquired using the 6546 QTOF. Samples were analyzed using Agilent's Profinder and RStudio. 


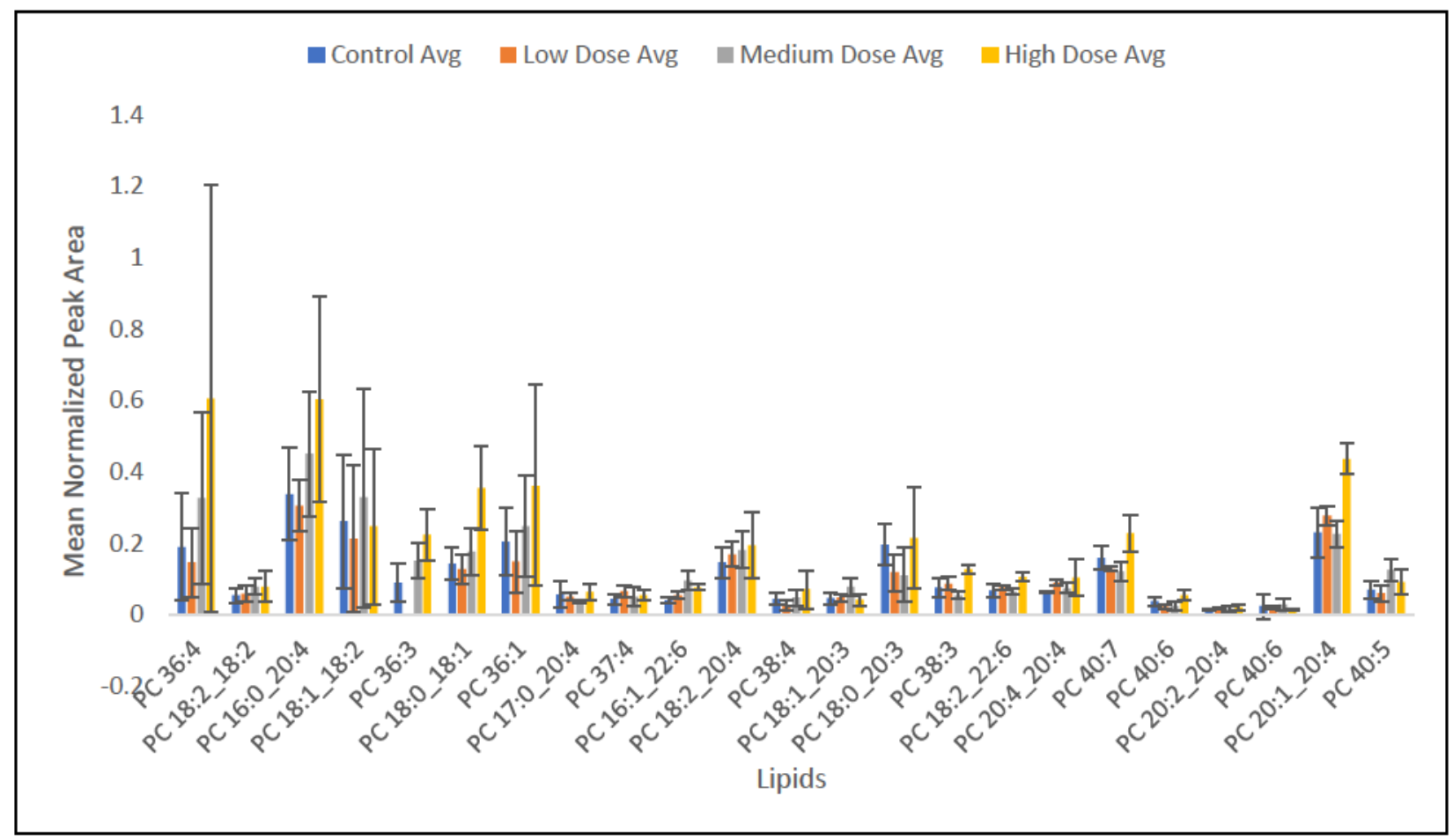

Figure S5: Histogram plot of mean normalized intensity lipids that were acquired using the 6546 QTOF. Samples were analyzed using Agilent's Profinder and RStudio.

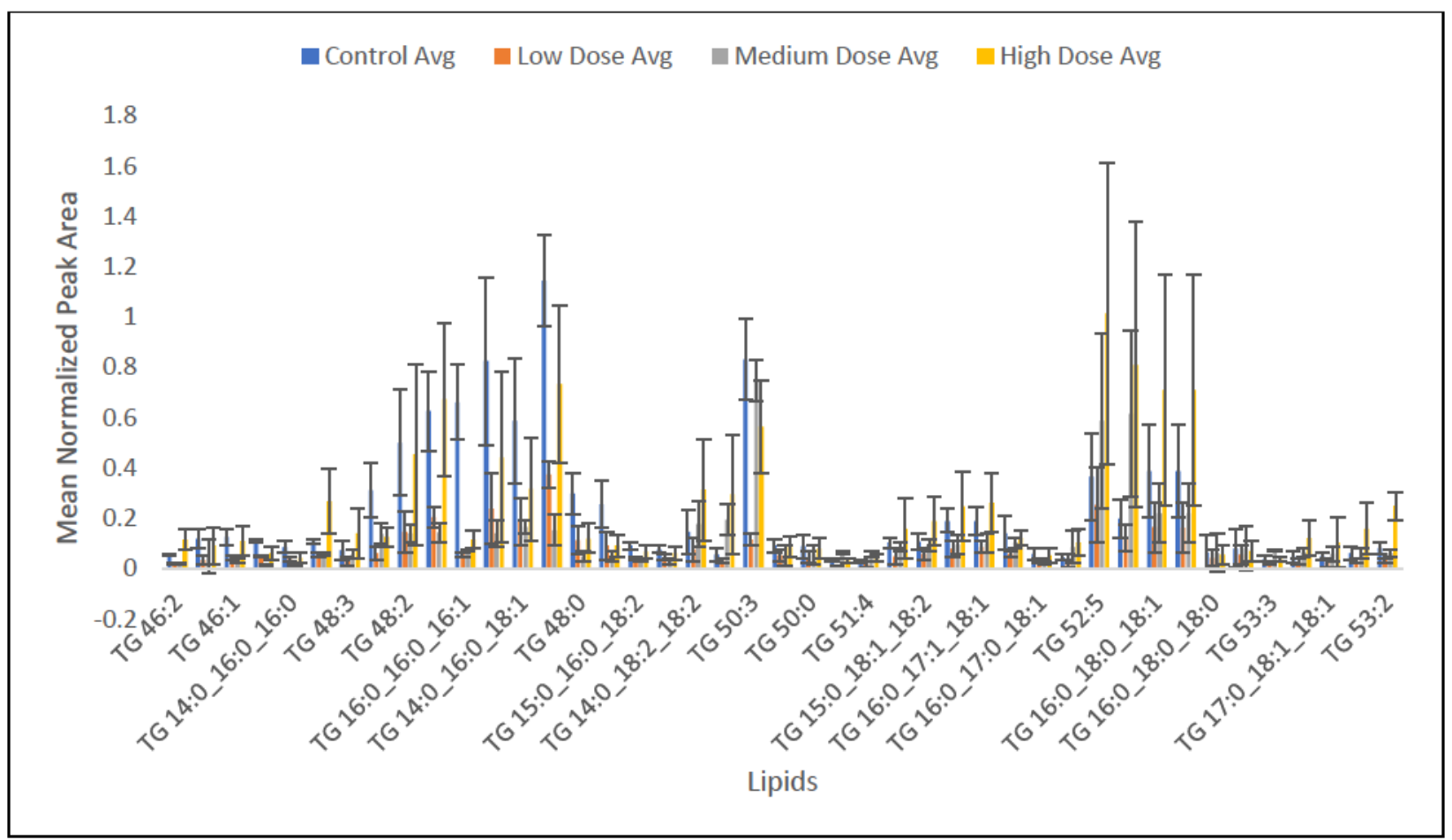

Figure S6: Histogram plot of mean normalized intensity lipids that were acquired using the 6546 QTOF. Samples were analyzed using Agilent's Profinder and RStudio. 


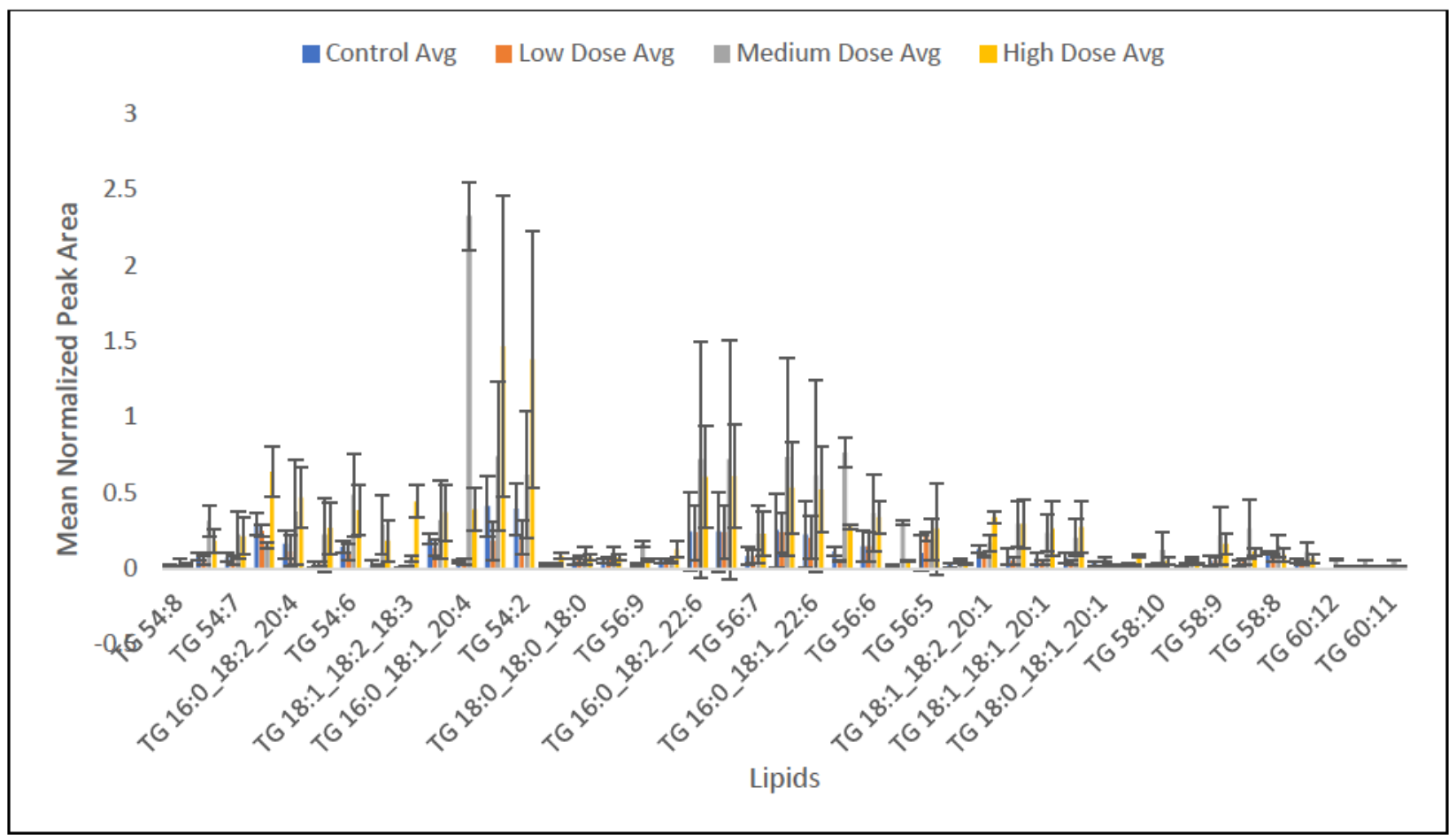

Figure S7: Histogram plot of mean normalized intensity lipids that were acquired using the 6546 QTOF. Samples were analyzed using Agilent's Profinder and RStudio.

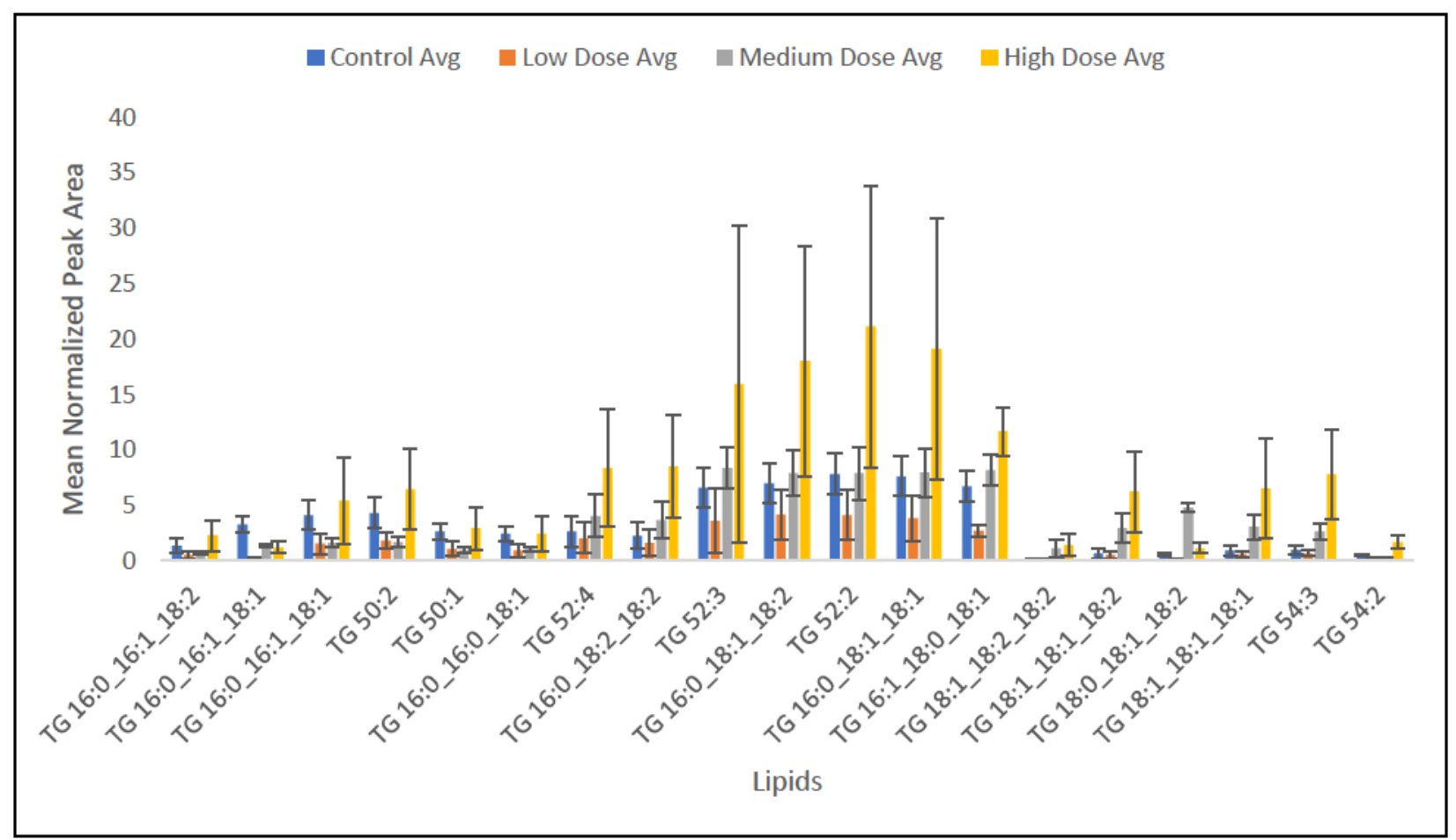

Figure S8: Histogram plot of mean normalized intensity lipids that were acquired using the 6546 QTOF. Samples were analyzed using Agilent's Profinder and RStudio. 